Copyright

by

Thomas Joseph Carroll III

2019 
The Dissertation Committee for Thomas Joseph Carroll III certifies that this is the approved version of the following dissertation:

\section{Three-Flavor Neutrino Oscillations with MINOS+}

Committee:

Karol Lang, Supervisor

Timothy Andeen

Karl Gebhardt

Peter Onyisi

Jack Ritchie 


\title{
Three-Flavor Neutrino Oscillations with MINOS+
}

\author{
by \\ Thomas Joseph Carroll III
}

\author{
DISSERTATION \\ Presented to the Faculty of the Graduate School of \\ The University of Texas at Austin \\ in Partial Fulfillment \\ of the Requirements \\ for the Degree of \\ DOCTOR OF PHILOSOPHY
}

THE UNIVERSITY OF TEXAS AT AUSTIN

August 2019 
Dedicated to my parents. 


\section{Acknowledgments}

The completion of this dissertation would not have been possible without help from many people along the way.

I am particularly grateful for the support and guidance from my advisor, Karol Lang. His passion and attention to detail will serve as an example as I work toward my professional goals.

I am thankful for the time I got to spend with the UT group which was an important part of my experiences at UT and Fermilab. I especially need to thank Adam Schreckenberger, Simon De Rijck, Dung Duc Phan, Beatriz Tapia Oregui, Will Flanagan, Ramon Salazar, Junting Huang, and Marek Proga.

I need to thank my MINOS, MINOS+, and NOvA colleagues who provided invaluable help and advice as I worked on this dissertation. Adam Aurisano, Andy Blake, Rui Chen, Justin Evans, Robert Hatcher, Anna Holin, Jim Hylen, Art Kreymer, Adam Lister, Diana Méndez, Joe O’Connor, Alex Radovic, Alex Sousa, Jacob Todd, Jenny Thomas, and Leigh Whitehead, I could not have accomplished this without you.

Finally, I want to thank my friends and family who provided so much love and support along the way. Mom and Dad, thank you for everything you have done for me. Your love and encouragement made all of this possible. 


\title{
Three-Flavor Neutrino Oscillations with MINOS+
}

\author{
Publication No. \\ Thomas Joseph Carroll III, Ph.D. \\ The University of Texas at Austin, 2019 \\ Supervisor: Karol Lang
}

This dissertation presents the analysis of the last two years of data from the MINOS+ long-baseline neutrino oscillation experiment. The analysis explores the data above the first oscillation maximum for neutrinos in the standard three-flavor neutrino oscillation model, adding significantly more neutrino events and constraining the model with increased precision. The analysis of the high-energy region of the MINOS+ data set, where previously there has been limited study, can help constrain alternative models or possibly show evidence of new phenomena.

The predecessor to MINOS + , the MINOS experiment, measured $v_{\mu}$ disappearance and $v_{\mathrm{e}}$ appearance using Fermilab's NuMI $\nu_{\mu}$ beam from 2005 to 2012. During this period the neutrino beam's energy spectrum was focused to peak near the first oscillation maximum. In addition to measuring accelerator beam neutrinos, the MINOS Far Detector collected a sample of atmospheric neutrinos from 2003 to 2011. With these two samples, MINOS measured the 
atmospheric mass splitting, $\Delta m_{32}^{2}$, at the $5 \%$ level and the value of $\sin ^{2} \theta_{23}$ at the $15 \%$ level. The data also constrained the $\mathrm{CP}$ violating parameter $\delta_{\mathrm{CP}}$.

The MINOS+ experiment exposed the MINOS detectors to a neutrino beam peaked at energies above the oscillation maximum from 2013 to 2016. With this higher energy neutrino beam, MINOS+ measures the shape of the $v_{\mu}$ survival probability away from the oscillation maximum with unprecedented precision. Measuring the shape of the oscillation probability as a function of neutrino energy is an essential test of the three-flavor oscillation model. At these higher energies where the standard oscillation probability decreases, the neutrino energy spectrum is sensitive to potential perturbations from mixing with additional sterile neutrinos or non-standard neutrino interactions.

This analysis of the complete data set from MINOS+ finds no significant deviations from the three-flavor oscillation probability in the energy region of 4 to $10 \mathrm{GeV}$ covered by the neutrino beam. This provides increased confidence in the three-flavor model in this region and provides new constraints of the atmospheric oscillation parameters, $\Delta m_{32}^{2}$ and $\sin ^{2} \theta_{23}$, by combining the MINOS and MINOS+ data sets.

The hierarchy of the neutrino masses is related to the sign of $\Delta m_{32}^{2}$. When $\Delta m_{32}^{2}>0$, the mass hierarchy is classified as normal. When $\Delta m_{32}^{2}<0$, the mass hierarchy is classified as inverted. This analysis cannot rule out either of the mass hierarchies. For the normal mass hierarchy, the combined analysis measures $\left|\Delta m_{32}^{2}\right|=2.41 \pm 0.09 \times 10^{-3} \mathrm{eV}^{2}$ at $68 \%$ C.L. and $\sin ^{2} \theta_{23}=0.42_{-0.06}^{+0.23}$ at $90 \%$ C.L. For the inverted mass hierarchy, this analysis measures $\left|\Delta m_{32}^{2}\right|=$ 
$2.47_{-0.10}^{+0.08} \times 10^{-3} \mathrm{eV}^{2}$ at $68 \%$ C.L. and $\sin ^{2} \theta_{23}=0.42_{-0.06}^{+0.23}$ at $90 \%$ C.L.

In addition to the analysis and measurement of the atmospheric oscillation parameters using MINOS+, this work updates the constraint on $\delta_{\mathrm{CP}}$ from MINOS and performs an exploratory combination using $\boldsymbol{v}_{\mu}$ disappearance data from the NOvA experiment. The NOvA experiment uses the NuMI beam in an off-axis strategy that results in a narrow flux of muon neutrinos at the first oscillation maximum and allows precision measurements of the three-flavor parameters. Since NOvA and MINOS+ shared the same neutrino beam, their data can be combined taking advantage of common beam related uncertainties. Combined, the data from the MINOS, MINOS+, and NOvA experiments precisely map $v_{\mu}$ oscillation probabilities using the same neutrino beam in a way that could not be attempted before. 


\section{Table of Contents}

Acknowledgments $\quad$ v

Abstract vi vi v v v

List of Tables $\quad$ xii

List of Figures $\quad$ Xv

Chapter 1. Neutrino Oscillations $\quad 1$

1.1 Neutrinos . . . . . . . . . . . . . . . . . . . . . 1

1.2 Evidence for Neutrino Oscillations . . . . . . . . . . . . 3

1.3 Neutrino Mixing and Oscillations . . . . . . . . . . . . 5

1.3.1 Neutrino Mixing . . . . . . . . . . . . . 6

1.4 Neutrino Oscillations Formalism . . . . . . . . . . . . . . 7

1.5 Measuring Three-Flavor Oscillation Parameters . . . . . . . . 9

1.6 Open Questions in Neutrino Oscillation Physics . . . . . . . . 11

1.7 Accelerator Neutrino Experiments . . . . . . . . . . . . . . . 12

Chapter 2. The MINOS+ Experiment 14

2.1 NuMI Neutrino Beam . . . . . . . . . . . . . . . 16

2.2 The MINOS Detectors . . . . . . . . . . . . . . 22

2.2.1 Far Detector . . . . . . . . . . . . . . . 24

2.2 .2 Near Detector . . . . . . . . . . . . . . . . . 28

2.2.3 Cosmic Ray Veto Shield . . . . . . . . . . . . . . . . . . 29

2.3 Events and Reconstruction . . . . . . . . . . . . . . 31

$\begin{array}{lll}\text { Chapter 3. } & \text { The Data Set } & 38\end{array}$

3.1 Beam Exposure . . . . . . . . . . . . . . . . 38

3.2 Atmospheric Exposure . . . . . . . . . . . . . . . . . 40

3.3 Summary...................... . . 41 
Chapter 4. MINOS+ Event Selection 44

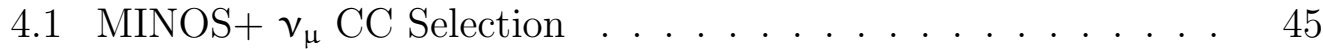

4.1 .1 Fiducial Volumes . . . . . . . . . . . . . . . . . . . 49

4.1 .2 Preselection . . . . . . . . . . . . . . . . . 50

4.1 .3 Selection . . . . . . . . . . . . . . 55

4.1.4 MINOS+ Selection Results . . . . . . . . . . . 58

4.2 Atmospheric Neutrino Selection . . . . . . . . . . . . 61

4.2 .1 CV $v_{\mu}$ Events . . . . . . . . . . . . . 61

4.2 .2 Neutrino Induced Muon Events . . . . . . . . . . . . . 64

4.2 .3 CV Shower Events . . . . . . . . . . . . . . . . 67

4.3 MINOS Non-Fiducial $v_{\mu}$ Selection . . . . . . . . . . . 68

4.4 MINOS $v_{\mathrm{e}}$ Selection . . . . . . . . . . . . . . . 71

$\begin{array}{lll}\text { Chapter 5. } & \text { MINOS+ Neutrino Flux } & 72\end{array}$

5.1 Hadron Production . . . . . . . . . . . . . . . . 74

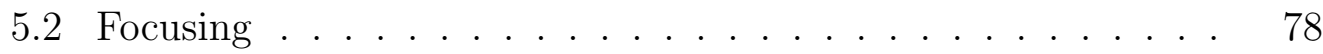

5.2 .1 Focusing Systematic Uncertainties _... . . . . . . 80

5.2 .2 Summary of Focusing Systematics _ . . . . . . . . 88

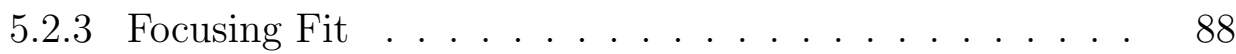

5.3 Run 13 Horn Tilt . . . . . . . . . . . . . . . . . . . . 89

5.4 Results . . . . . . . . . . . . . . . . . . . 95

$\begin{array}{lll}\text { Chapter 6. The Three-Flavor Analysis } & 99\end{array}$

6.1 Beam Analysis Strategy . . . . . . . . . . . . . . . . . . . . 99

6.2 Unfolding . . . . . . . . . . . . . . . . . . 100

6.3 Extrapolation: Beam Matrix . . . . . . . . . . . . . . . 105

6.4 Folding . . . . . . . . . . . . . . . . . . . . 109

6.5 Atmospheric neutrinos . . . . . . . . . . . . . . . . 113

6.6 The Fit to the Oscillation Parameters . . . . . . . . . . . . . 114

6.6 .1 Fit Statistic . . . . . . . . . . . . . . . . 115

6.6.2 Independent Constraints . . . . . . . . . . . . . . . . 116

6.7 Systematics . . . . . . . . . . . . . . . . 116

6.7.1 Beam Systematic Uncertainties . . . . . . . . . . . 116 
6.7.2 Atmospheric Systematic Uncertainties . . . . . . . . . 119

6.7.3 Correlated Systematics . . . . . . . . . . . . . . . 125

6.7.4 Summary of Systematic Uncertainties . . . . . . . . 127

6.8 Measuring $\Delta m_{32}^{2}$ and $\sin ^{2} \theta_{23} \ldots \ldots$. . . . . . . . . . . . . . 130

Chapter 7. Results 133

7.1 MINOSt . . . . . . . . . . . . . . . . 133

7.2 MINOS and MINOS+ ................. . . 137

7.2 .1 Measurement of $\Delta m_{32}^{2}$ and $\sin ^{2} \theta_{23} \ldots \ldots \ldots 138$

$7.2 .2 \delta_{\mathrm{CP}}$ Constraints . . . . . . . . . . . . . . 143

7.2.3 Combining MINOS and MINOS+ with NOvA . . . . 145

$\begin{array}{lll}\text { Chapter 8. Summary } & 148\end{array}$

$\begin{array}{lr}\text { Appendices } & 150\end{array}$

Appendix A. MINOS+ Run 12 and Run 13 Fit 151

$\begin{array}{lll}\text { Appendix B. } & \text { MINOS+ Fit } & 159\end{array}$

$\begin{array}{lll}\text { Appendix C. } & \text { MINOS and MINOS+ Fit } & 163\end{array}$

$\begin{array}{lr}\text { Bibliography } & 169\end{array}$

$\begin{array}{lr}\text { Vita } & 186\end{array}$ 


\section{List of Tables}

3.1 MINOS and MINOS+ beam exposure at the FD by run period along with each run configuration $[65,66]$. The neutrino beam energy is classified as LE for low-energy, ME for mediumenergy, and $\mathrm{HE}$ for high-energy. . . . . . . . . . . . . .

3.2 MINOS and MINOS+ atmospheric neutrino exposures at the FD $[56,67,68] \ldots \ldots \ldots \ldots \ldots$

3.3 Distinct MINOS and MINOS+ exposures used in the threeflavor analysis. The neutrino beam energy is classified as LE for low-energy, ME for medium-energy, and HE for high-energy.

5.1 MINOS+ beam focusing systematics. Omitting the baffle scrapping systematic which was found to be negligible. . . . . . .

6.1 MINOS+ Run XII total purities for selecting $v_{\mu}$ or $\bar{v}_{\mu}$ CC events.101

6.2 MINOS+ Run XII total efficiencies for selecting $v_{\mu}$ or $\bar{v}_{\mu} \mathrm{CC}$ events. . . . . . . . . . . . . . . . 105

6.3 Earth density profile used to account for matter effects in the atmospheric neutrino oscillation analysis. . . . . . . . . . . . 114

6.4 MINOS and MINOS+ beam sample $1 \sigma$ systematic uncertainty values. . . . . . . . . . . . . . . . . . . . . . . . . . 127

6.5 Atmospheric neutrino sample $1 \sigma$ systematic uncertainty values. 128

6.6 MINOS and MINOS+ beam and atmopsheric systematic uncertainty best fit values, accounting for correlations. . . . . . . 130

7.1 Number of events selected in each sample for the combined MINOS and MINOS+ data compared to the no oscillations and best fit predictions. . . . . . . . . . . . . . . .

7.2 MINOS and MINOS+ combined best fit parameters for each mass hierarchy and $\theta_{23}$ octant. The value of $-2 \Delta \log (\mathcal{L})$ calculated relative to the overall best fit point is provided for each combination. . . . . . . . . . . . .

7.3 MINOS, MINOS+ mass hierarchy confidence limits. $\left|\Delta m_{32}^{2}\right|$ is in units of $10^{-3} \mathrm{eV}^{2} \ldots \ldots \ldots \ldots$. . . . . . . . . . . 
7.4 MINOS, MINOS+ normal mass hierarchy octant confidence limits calculated from the 1D profiles with the fit preferences for lower octant and non-maximal mixing. . . . . . . . . .

7.5 MINOS, MINOS+ inverted mass hierarchy octant confidence limits calculated from the 1D profiles with the fit preferences for lower octant and non-maximal mixing. . . . . . . . . . .

A.1 Runs 12 and 13 event counts of $v_{\mu}$ from $v_{\mu}$-mode beam. . . . 154

A.2 Runs 12 and 13 event counts of $\bar{v}_{\mu}$ from $v_{\mu}$-mode beam. . . . 154

A.3 MINOS+ Runs 12 and 13 combined best fit parameters for each mass hierarchy and $\theta_{23}$ octant. The value of $-2 \Delta \log (\mathcal{L})$ calculated relative to the overall best fit point is provided for each combination. . . . . . . . . . . . . . .

A.4 Runs 12 and 13 mass hierarchy confidence limits. $\left|\Delta m_{32}^{2}\right|$ is in units of $10^{-3} \mathrm{eV}^{2}$. . . . . . . . . . . . . . . .

A.5 Runs 12 and 13 normal mass hierarchy octant confidence limits calculated from the 1D profiles with the fit preferences for lower octant and non-maximal mixing. . . . . . . . . . . . . .

A.6 Runs 12 and 13 inverted mass hierarchy octant confidence limits calculated from the 1D profiles with the fit preferences for lower octant and non-maximal mixing. . . . . . . . . . . . . .

B.1 MINOS + event counts of $v_{\mu}$ from $v_{\mu}$-mode beam. The predicted number of events in the best fit column of the table come from fitting the measured $v_{\mu}$ and $\bar{v}_{\mu}$ events from the full MINOS+ data set. . . . . . . . . . . . . .

B.2 MINOS+ event counts of $\bar{v}_{\mu}$ from $v_{\mu}$-mode beam. The predicted number of events in the best fit column of the table come from fitting the measured $\nu_{\mu}$ and $\bar{v}_{\mu}$ events from the full MINOS+ data set. . . . . . . . . . . . . .

B.3 MINOS+ best fit parameters for each mass hierarchy and $\theta_{23}$ octant. The value of $-2 \Delta \log (\mathcal{L})$ calculated relative to the overall best fit point is provided for each combination. . . . . . .

B.4 MINOS+ mass hierarchy confidence limits. $\left|\Delta m_{32}^{2}\right|$ is in units of $10^{-3} \mathrm{eV}^{2}$. . . . . . . . . . . . . . . . . . . 162

B.5 MINOS+ normal mass hierarchy octant confidence limits calculated from the 1D profiles with the fit preferences for lower octant and non-maximal mixing. . . . . . . . . . . . . . . .

B.6 MINOS+ inverted mass hierarchy octant confidence limits calculated from the 1D profiles with the fit preferences for lower octant and non-maximal mixing. . . . . . . . . . . . 
C.1 MINOS and MINOS+ event counts of $v_{\mu}$ from $v_{\mu}$-mode beam. The predicted number of events in the best fit column of the table come from fitting the measured $v_{\mu}$ and $\bar{\nu}_{\mu}$ events from the full MINOS and MINOS+ data set. . . . . . . . . . . . . .

C.2 MINOS and MINOS+ event counts of $\bar{v}_{\mu}$ from $v_{\mu}$-mode beam. The predicted number of events in the best fit column of the table come from fitting the measured $v_{\mu}$ and $\bar{v}_{\mu}$ events from the full MINOS and MINOS+ data set. . . . . . . . . . . .

C.3 MINOS event counts of $\bar{v}_{\mu}$ from $\bar{v}_{\mu}$-mode beam. The predicted number of events in the best fit column of the table come from fitting the measured $v_{\mu}$ and $\bar{v}_{\mu}$ events from the full MINOS and MINOS+ data set. . . . . . . . . . . . .

C.4 MINOS event counts of nonfiducial muons from $v_{\mu}$-mode beam. The predicted number of events in the best fit column of the table come from fitting the measured $v_{\mu}$ and $\bar{v}_{\mu}$ events from the full MINOS and MINOS+ data set. . . . . . . . . . . .

C.5 MINOS and MINOS+ event counts of CV atmospheric events. The predicted number of events in the best fit column of the table come from fitting the measured $\nu_{\mu}$ and $\bar{v}_{\mu}$ events from the full MINOS and MINOS+ data set. . . . . . . . . .

C.6 MINOS and MINOS+ event counts of nonfiducial neutrino induced muon (rock) atmospheric events. The predicted number of events in the best fit column of the table come from fitting the measured $v_{\mu}$ and $\bar{v}_{\mu}$ events from the full MINOS and MINOS+ data set. . . . . . . . . . . . . . 


\section{List of Figures}

1.1 Measurements of the hadron production cross section around the $\mathrm{Z}$ resonance by the LEP experiments [5]. The curves are the predicted cross sections for two, three, and four neutrinos with Standard Model couplings and negligible mass. . . . . . .

1.2 Diagrams of charged-current (left) and neutral-current (right) vertices involving neutrinos of flavor $\alpha, \nu_{\alpha}$, and corresponding charged lepton, $l_{\alpha}^{-}$. These diagrams were made using TikZFeynman [8]. . . . . . . . . . . . . . . . . . . . . . .

1.3 Neutrino spectrum on-axis for MINOS+ and off-axis for NOvA. The plot also shows the lower energy spectrum produced on-axis for the predecessor to MINOS+, MINOS. . . . . . . . . . . .

2.1 The MINOS and MINOS+ experimental setup. The map above illustrates the $735 \mathrm{~km}$ baseline from Fermilab to Soudan, MN where the MINOS far detector was located. The drawing on the bottom summarizes the masses, positions, and sizes relative to the neutrino beam of the MINOS detectors. . . . . . . . .

2.2 Cartoon of the NuMI neutrino beamline. . . . . . . . . . . .

2.3 The MINOS era NuMI baffle. On the left is the baffle by itself. On the right is the baffle connected to the target module, when installed in the beamline the baffle and target are one unit. These drawing are from Ref. [42]. . . . . . . . . . . . . . .

2.4 Drawing of the NuMI target hall showing Horn 2 at different positions for the low, medium, and high-energy beam configurations. The horn positions are labeled in inches. Drawing from Ref. [47]. . . . . . . . . . . . . . .

2.5 MINOS low-energy and high-energy $v_{\mu}$ CC spectra at the ND compared to the MINOS+ medium-energy $v_{\mu}$ CC spectrum. .

2.6 The NuMI low-energy and medium-energy targets. On the left is the target used by MINOS which was designed to be inserted into the upstream focusing horn. On the right is the target used by MINOS+ which was designed for a $700 \mathrm{~kW}$ proton beam. The diagrams adapted from Refs. $[42,48] \ldots$. . . . . . . . . 
2.7 Cartoon of neutrino mode, Forward Horn Current, focusing of the mesons to produce the neutrino beam. In Forward Horn Current mode the horn current on the inner conductor of the horns flows in the same direction as the beam. Drawing from Ref. [40]. . . . . . . . . . . . . . . .

2.8 Comparison of $\boldsymbol{v}_{\mu}$ (black) and $\bar{v}_{\mu}$ (red) spectra for low-energy (left) and medium-energy (right) NuMI beam at the MINOS near detector. Figures from Ref. [49]. . . . . . . . . . . . .

2.9 Cutaway drawing of a MINOS detector scintillator strip. Light produced by an ionizing particle is multiply reflected by the reflective titanium-dioxide coating. Light absorbed by the wavelength shifting fiber is re-emitted isotropically. Wavelength shifted photons whose directions fall within the total internal reflection cones are transported along the fiber to the edge of the detector in order to be readout. Drawing from Ref. [50] and made by M. Proga. . . . . . . . . . . . . . . . . . . .

2.10 Schematic drawing of the readout of a scintillator module. An edge of a detector plane is on right side of the sketch is exaggerated to show how the scintillator strips are readout. The wavelength shifting fibers from the strips are coupled to clear optical fibers which carry the light to a pixel of the photomultiplier tube. Drawing from Ref. [50] and made by M. Proga. .

2.11 The MINOS far detector. On the left is a labeled drawing of the detector pictured on the right. This perspective is looking south toward Fermilab from the end of the second supermodule. "A" is the furthest downstream steel plane of the detector. "B" is part of the cosmic ray veto shield which is both above and along the sides of the detector. "C" is the end of the magnet coil. "D" is an electronics rack on one of the elevated walkways alongside the detector. This figure is from Ref. [50]. . . . . . .

2.12 The magnetic field in the far detector (left) and near detector (right) courtesy of Robert Hatcher. These are from the perspective of looking toward the NuMI target. The $z$-axis of the histograms are in units of $\mathrm{T}$. The features seen in the far detector plane are from the seams of the steel plates that were assembled to form a single far detector plane. . . . . . . . . .

2.13 Layout of $U$ (left) and $V$ (right) modules on far detector planes. $U$ - and $V$-type planes were interleaved. "A" and "B" module types have 28 scintillator strips and the other types have 20 strips. The first (upstream) scintillator plane of each supermodule was of the $V$-type. This figure is from Ref. [50]. . . . . 
2.14 The MINOS near detector. On the left is a labeled drawing of the detector pictured on the right. This perspective is looking in the direction of the neutrino beam toward the far detector location. "A" is the furthest upstream steel plane of the detector. "B" is the magnet coil. "C" is an electronics rack on the elevated walkway. This figure is from Ref. [50]. . . . . . . . . .

2.15 The four scintillator plane configurations used in the MINOS near detector. The planes are the top are referred to as partial views, and the planes on the bottom are referred to as full views. The $U$-view planes are on the left, and the $V$-view planes are on the right. The individual panels have a label from $\mathrm{G}$ to $\mathrm{N}$ to denote different shape panels used to construct the plane configuration. Full and partial view configurations used in the ND active planes. The partial view planes are oriented so that they cover the beam center. The full view planes cover the beam center and extend beyond the coil hole of the detector. Drawing from Ref. [50]. . . . . . . . . . . . . .

2.16 Schematic of the Far Detector cosmic ray veto shield looking toward Fermilab. From Ref. [50]. . . . . . . . . . . . .

2.17 Plot of the average veto shield efficiency measured using stopping cosmic-ray muons as a function of time for the complete exposure period. The points shown in blue correspond to the previously analyzed data while the red points represent the new data from February 2014 to June 2016. The average efficiency is calculated to be $96.2 \%$. Figure from Ref. [56]. . . . . . . . .

2.18 Event display of a simulated $v_{\mu}$ charged-current interaction in a MINOS detector. The display shows the characteristic bending muon track of a $v_{\mu}$ charged current interaction. Event display from [57]. . . . . . . . . . . . . . . . . .

2.19 Event display of a simulated neutral-current interaction in a MINOS detector. The display shows the hadronic shower characteristic of neutral current interactions. Event display from [57]. 33

2.20 Event display of a simulated $v_{\mathrm{e}}$ charged-current interaction in a MINOS detector. Charged current $v_{\mathrm{e}}$ interactions produce a shower in the detector like neutral current interactions; however, these showers have an electromagnetic component in addition to the hadronic activity. Event display from [57]. . . . . . . . 
3.1 The total number of protons on the NuMI target over both the MINOS and MINOS+ runs. The plot covers the entire running time from 2005 to 2016. MINOS took beam data in different configurations: low-energy $v_{\mu}$-mode (green), low-energy $\bar{v}_{\mu}$-mode (orange), and special configurations (red) which include high-energy $\bar{v}_{\mu}$-mode or the focusing horns turned off. The medium-energy $v_{\mu}$-mode POT for MINOS+ is shown in pink. The individual configurations are plotted with the POT per week, and the cumulative amount of POT is plotted in blue. 39

3.2 The total number of protons on the NuMI target during MINOS+. The plot covers the entire MINOS+ running from 2013 to 2016. The POT per week is plotted in green, and the cumulative amount of POT is plotted in blue. . . . . . . . . . .

3.3 Combined MINOS $v_{\mu}$ CC energy spectrum from the $v_{\mu}$-mode beam at the ND compared to the $v_{\mu}$ CC spectra from the MINOS+ runs. The individual MINOS low-energy run spectra are stacked solid histograms. The MINOS high-energy spectrum is multiplied by a factor of 10 and shown in purple. The MINOS+ run spectra are plotted individually as the line histograms in green, blue, and red. . . . . . . . . . . . .

3.4 The number of selected atmospheric neutrino events at the FD per live-day of running, for the full duration of data taking. The points shown in blue correspond to the previously analyzed data while the red points represent the new data (February 2014 to June 2016). Events passing the selection cuts occur at a mean rate of 0.80 per live-day. This figure is from Ref. [56]. . . . . .

4.1 MINOS $+k \mathrm{NN}$ selection variable distributions from the ND training sample for $v_{\mu}$ CC signal events and background events.

4.2 MINOS $+k \mathrm{NN}$ selection variable distributions from the FD training sample for $v_{\mu}$ CC signal events and background events.

4.3 Distribution of the $v_{\mu}$-CC event vertex coordinates in the ND for the MINOS+ beam exposure. The $X$ distribution (top left), $Y$ distribution (top right), and $Z$ distribution (bottom) of data and $\mathrm{MC}$ events. $Z=0$ corresponds to the first plane in the detector. . . . . . . . . . . . . . .

4.4 Distribution of the $\bar{v}_{\mu}$-CC event vertex coordinates in the ND for the MINOS+ beam exposure. The $X$ distribution (top left), $Y$ distribution (top right), and $Z$ distribution (bottom) of data and $\mathrm{MC}$ events. $Z=0$ corresponds to the first plane in the detector. . . . . . . . . . . . . . . 
4.5 Distribution of the $v_{\mu}$-CC event vertex coordinates in the FD for the MINOS+ beam exposure. The $X Y$-view of the vertices for data events is plotted on the top left. The $Z$-plane distribution (top right), $X$ distribution (bottom left), and $Y$ distribution (bottom right) of data and oscillated MC events. . . . . . . . .

4.6 Distribution of the $\bar{v}_{\mu}$-CC event vertex coordinates in the FD for the MINOS+ beam exposure. The $X Y$-view of the vertices for data events is plotted on the top left. The $Z$-plane distribution (top right), $X$ distribution (bottom left), and $Y$ distribution (bottom right) of data and oscillated MC events. . . . . . . . .

4.7 Distribution of the cosine of the angle between the $\mu^{-}$track and the beam direction for selected $v_{\mu}$ events in data and oscilalted MC. . . . . . . . . . . . . . . .

4.8 Distribution of the cosine of the angle between the $\mu^{+}$track and the beam direction for selected $\bar{v}_{\mu}$ events in data and oscillated MC. . . . . . . . . . . . . . ...

4.9 MINOS $+k \mathrm{NN}$ ND distribution of the selection parameter for selected $v_{\mu}-\mathrm{CC}$ events with expected distribution and expected $\mathrm{NC}$ contamination. Events with a separation parameter greater than 0.3 are selected. . . . . . . . . . . . . . . . .

4.10 MINOS $+k$ NN ND distribution of the selection parameter for selected $\bar{v}_{\mu}$-CC events with expected distribution and expected $\mathrm{NC}$ contamination. Events with a separation parameter greater than 0.3 are selected. . . . . . . . . . . . . . . .

4.11 MINOS+ CC selection efficiency and purity for $v_{\mu}$ and $\bar{v}_{\mu}$ interactions at the Near Detector (left) and Far Detector (right) as functions of neutrino energy. . . . . . . . . . . . .

4.12 MINOS+ selected $v_{\mu}$ and $\bar{v}_{\mu}$ CC spectrum at the Near Detector compared to the MC. . . . . . . . . . . . . . . .

4.13 The veto shield is used to remove the cosmic-ray muon background in the atmospheric contain-vertex event samples. For each event, the nearest hit in time is located in the sections of the shield above the vertex. The relative time distribution is plotted for a measured cosmic-ray muon sample. The time window of $\pm 50 \mathrm{~ns}$, indicated by the arrows, is used to veto cosmicray muons. From Ref. [56]. . . . . . . . . . . . 
4.14 Distributions of the trace variable, $\Delta_{Z}$, plotted for contained tracks satisfying the fiducial cuts. The trace is found by extending the track trajectory from the highest vertex back to the edge of the detector and calculating the displacement in $z$. Cosmic-ray muons typically enter the detector at a steep angle and travel a small distance before entering the scintillator. To reject the cosmic-ray muon background, a selection cut of $\Delta_{Z}>0.5 \mathrm{~m}$ is applied to contained-vertex muon tracks. From Ref. [56]. . . . . . . . . . . . . . . .

4.15 Distributions of maximum pulse height at the highest end of the track, $Q_{\mathrm{vtx}}$, plotted against the $y$-component $\left(\cos \theta_{y}\right)$ and $z$-component $\left(\cos \theta_{z}\right)$ of the downward direction. The distributions are plotted for those events that pass the prior trace and topology cuts and span $<25$ planes. The plots on the right show the cosmic-ray muon background, given by vetoed events in data; the plots on the left show Monte Carlo atmospheric neutrinos. The background events are associated with large vertex pulse heights and track directions parallel to the scintillator planes. The hatched regions are rejected by the cuts. From Ref. [56]. . . . . . . . . . . . . . . . .

4.16 Observed distribution of $1 / \beta$ variable, given for each event by the gradient of a linear fit to the measured times as a function of their distance on the track. The distribution is plotted for upward and downward muons that pass the timing cuts. A good separation is achieved between the upward-going neutrinoinduced muon signal, which is peaked at +1.0 , and the downwardgoing cosmic muon background, which is peaked at -1.0. Note that, with a log scale, the peaks appear asymmetric. This is due to the selection cuts, which disfavor $1 / \beta=0$. The arrow indicates upward tracks. From Ref. [56]. . . . . . . . . .

4.17 Distribution of reconstructed cosine of the zenith angle for muons, with good timing and topology. In the range $0.10<\cos \theta_{\text {zen }}<$ 0.30 , the observed rate of muons is dominated by the cosmic muon background and falls steeply as the rock overburden increases rapidly. For $\cos \theta_{\text {zen }}<0.10$, the distribution flattens, as the cosmic muon flux falls below that of neutrino-induced muons. To minimize the background from cosmic-ray muons, a cut is placed at $\cos \theta_{\text {zen }}<0.05$. From Ref. [56]. . . . . . . . 
4.18 Distributions of the trace variable, $\Delta_{Z}$, calculated for containedvertex showers. The hatched histogram shows the Monte Carlo prediction for atmospheric neutrinos. The solid line gives the overall expectation, dominated by cosmic muon background, which is obtained by scaling vetoed events in the data by the measured cosmic-ray veto shield efficiency. Typically, the cosmicray muon background events enter the detector at a small angle to the planes and travel a small distance along the $z$ direction before passing into the scintillator. A selection cut of $\Delta_{Z}>0.8 \mathrm{~m}$ is applied to separate signal from background. From Ref. [56]. . . . . . . . . . . . . . . . .

4.19 Distributions of mean and RMS shower pulse height per plane for contained-vertex showers satisfying the shower trace cuts. Background events from cosmic rays have a larger mean and RMS, particularly for short showers. In order to separate signal and background, events are divided into short ( $\leq 8$ planes) and long ( $>8$ planes) showers. The plots show the distributions for each of these samples, and the cuts applied. From Ref. [56].

5.1 The $v_{\mu}$ Near Detector energy spectra for six different beam configurations during the MINOS experiment. The spectra are compared to the default flux simulation using FLUKA05 and the flux simulation tuned using the Near Detector neutrino data. The ratio of the data to $\mathrm{MC}$ prediction is shown beneath each spectrum. From Ref. [40]. . . . . . . . . . . . . . .

5.2 Production yields for $\pi^{+}$as functions of transverse momentum for different values of $x_{F}$. Courtesy of Anna Holin. . . . . . . .

5.3 The best fits for $B\left(x_{F}\right), C\left(x_{F}\right), D\left(x_{F}\right)$, and $E\left(x_{F}\right)$ for $\pi^{+}$. From

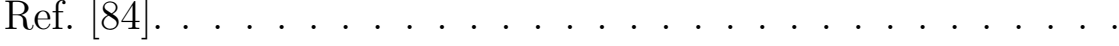

5.4 The $\pi^{+}$hadron production weights after fitting the horn off ND data. From Ref. [84]. . . . . . . . . . . . . . . . .

5.5 The measured ND horn off $v_{\mu}$ CC spectrum compared to the the MC before (blue) and after (red) the hadron production fit. From Ref. [84]. . . . . . . . . . . . . . . . . . . .

5.6 The measured ND horn on $v_{\mu}$ CC spectrum compared to the the $\mathrm{MC}$ before (blue) and after (red) the hadron production fit. From Ref. [84]. . . . . . . . . . . . . . . . . . .

5.7 The 10kA horn current miscalibration focusing uncertainty for muon neutrinos on the nominal 200kA horn current. . . . . . .

5.8 Horn material uncertainty for muon neutrinos calculated as $5 \%$ of the result of replace the horns in the flux simulation with air. 
5.9 Horn current distribution uncertainty for muon neutrinos. . .

84

5.10 Horn 1 alignment uncertainty for muon neutrinos. Plot from Ref. [89]. . . . . . . . . . . . . . .

5.11 Proton beam position uncertainty for muon neutrinos. Plot from Ref. [89]. . . . . . . . . . . . . . 86

5.12 Proton beam width uncertainty for muon neutrinos. . . . . . . 87

5.13 Number of protons on target uncertainty for muon neutrinos. . 88

5.14 Baffle scraping uncertainty for muon neutrinos. . . . . . . . . 89

5.15 The measured ND horn on $v_{\mu}$ CC spectrum compared to the the $\mathrm{MC}$ before (blue) and after (red) both the hadron production and focusing fits. From Ref. [84]. . . . . . . . . . . . .

5.16 Schematic drawing of the bushing that failed highlighted in red which allowed horn 1 to fall and tilt. The washer highlighted in blue was bent by the horn positioning rod initially being pulled up passed the bushing housing after the bushing failed. . . . .

5.17 The simulated flux at the MINOS ND when the upstream end of horn 1 is lowered by different angles. . . . . . . . . .

5.18 The ratio of the NuMI flux at the MINOS detectors for the upstream end of horn 1 lowered by $1.37 \mathrm{mrad}$ to the nominal flux. 94

5.19 Horn tilt systematic band used to fit the Run 13 horn tilt. . .

5.20 Near Detector $v_{\mu}$-CC event rate over time. The top plot shows the event rate for neutrino energy less than $8 \mathrm{GeV}$, and the bottom plot shows the event rate for neutrino energy greater than $8 \mathrm{GeV}$. . . . . . . . . . . . . .

5.21 Event rate in arbitrary units at the NuMI muon monitor in alcove 3 from October 2015 to May 2016. The plot shows the event rate increasing within the period from October to February. This time period corresponds to when NuMI Horn 1 is believed to have tilted. From Ref. [97]. . . . . . . . . . . . . .

5.22 Reconstructed neutrino energy for MINOS+ Run 11 events in the near detector. The data spectrum in black is compared to the tuned simulated spectrum with $1 \sigma$ flux uncertainties in red.

5.23 Reconstructed neutrino energy for MINOS+ Run 12 events in the near detector. The data spectrum in black is compared to the tuned simulated spectrum with $1 \sigma$ flux uncertainties in red.

5.24 Reconstructed neutrino energy for MINOS+ Run 13 events in the near detector. The data spectrum in black is compared to the tuned simulated spectrum with $1 \sigma$ flux uncertainties in red. 
6.1 MINOS+ Run 12 ND purity for selected $\boldsymbol{v}_{\mu}$ and $\bar{v}_{\mu}$ events as a function of reconstructed neutrino energy. . . . . . . . . .

6.2 MINOS+ Run 12 ND Reconstructed Neutrino Energy vs True Neutrino Energy for selected $\boldsymbol{v}_{\mu}$ (top) and $\bar{v}_{\mu}$ (bottom) events used to unfold the ND spectrum in true neutrino energy. . . .

6.3 MINOS+ Run 12 ND efficiency for selected $v_{\mu}$ and $\bar{v}_{\mu}$ events as a function of true neutrino energy. . . . . . . . . . . .

6.4 Illustration of the angular acceptance of the Near Detector and Far Detector. From Ref. [40]. . . . . . . . . . . . . . . . . . .

6.5 Illustration of the effect of the angular acceptance of the Near Detector and Far Detector on the observed flux. From Ref. [40]. 106

6.6 The beam matrix used for MINOS+ Run $12 v_{\mu}$ (top) and $\bar{v}_{\mu}$ (bottom) events. . . . . . . . . . . . 110

6.7 MINOS + Run 12 FD efficiency for selected $v_{\mu}$ and $\bar{v}_{\mu}$ events as function of true neutrino energy. . . . . . . . . . . . .

6.8 MINOS+ Run 12 FD Reconstructed Neutrino Energy vs True Neutrino Energy for selected $v_{\mu}$ (top) and $\bar{v}_{\mu}$ (bottom) events.

6.9 MINOS+ Run 12 FD purity for selected $v_{\mu}$ and $\bar{\nu}_{\mu}$ events as a function of reconstructed neutrino energy. . . . . . . . . .

6.10 The effects of altering the neutrino spectrum by shifting the beam systematic uncertainties by $\pm 1 \sigma$ on the statistics only fit of $\Delta m_{32}^{2}$ and $\sin ^{2} \theta_{23}$ for the combined MINOS and MINOS+ neutrino beam exposure. The left plot shows the effects of shift the MINOS and MINOS+ spectra together with fully correlated systematics. The top plot shows the effects of only systematically shifting the MINOS spectrum, and the bottom plot shows the effects of only systematically shifting the MINOS+ spectrum. From Ref. [125]. . . . . . . . . . . . . . . . .

6.11 The effects of altering the neutrino spectrum by shifting the beam and atmospheric systematic uncertainties by $\pm 1 \sigma$ on the statistics only fit of $\Delta m_{32}^{2}$ and $\sin ^{2} \theta_{23}$. The top plot shows the resulting shifts compared to the $2 \mathrm{D}$ confidence intervals in $\Delta m_{32}^{2}$ and $\sin ^{2} \theta_{23}$ for the combined MINOS and MINOS+ beam and atmospheric data samples. The bottom plot shows the relative changes in $\Delta m_{32}^{2}$ and $\sin ^{2} \theta_{23}$ from the best fit value as a result of shifting the systematic uncertainties. . . . . . . . . .

$6.12 \chi^{2}$ surface produced by the MINOS, MINOS+ fit for the normal mass hierarchy. . . . . . . . . . . . .

$6.13 \chi^{2}$ surface produced by the MINOS, MINOS+ fit for the inverted mass hierarchy. . . . . . . . . . . . . 
7.1 MINOS+ contained-vertex $v_{\mu}$ and $\bar{v}_{\mu}$ data spectrum and predictions at the far detector. The data spectrum is shown as black points. The orange curve is the predicted spectrum with no oscillations, and the best fit spectrum is shown in blue for $\Delta m_{32}^{2}=-2.51 \times 10^{-3} \mathrm{eV}^{2}$ and $\sin ^{2} \theta_{23}=0.62$. The predicted spectrum assuming the best fit measured using the full MINOS data set [64] is shown in green. . . . . . . . . . . .

7.2 MINOS + ratios of data and best fit spectra to the no oscillations prediction. The data ratio in black clearly shows muon neutrino disappearance. The green curve is the ratio for the prediction assuming the MINOS best fit from Ref. [64]. The ratio for the best fit at $\Delta m_{32}^{2}=-2.51 \times 10^{-3} \mathrm{eV}^{2}$ and $\sin ^{2} \theta_{23}=0.62$ is drawn in blue. . . . . . . . . . . . . . .

7.3 MINOS $+\Delta m_{32}^{2}$ and $\sin ^{2} \theta_{23}$ confidence limits. The left panel shows the $68 \%$ and $90 \%$ confidence limits on $\Delta m_{32}^{2}$ and $\sin ^{2} \theta_{23}$ for the normal mass hierarchy (top) and the inverted mass hierarchy (bottom). The best fit from the full MINOS+ beam exposure is plotted as a star. The right panels show the 1D likelihood profiles as functions of $\Delta m_{32}^{2}$ and $\sin ^{2} \theta_{23}$ for each mass hierarchy. . . . . . . . . . . . . . . . . . .

7.4 MINOS and MINOS+ combined contained-vertex $v_{\mu}$ and $\bar{v}_{\mu}$ data spectrum and predictions at the far detector. The data spectrum is shown as black points. The orange curve is the predicted spectrum with no oscillations, and the blue curve is the best fit to the data for $\Delta m_{32}^{2}=2.41 \times 10^{-3} \mathrm{eV}^{2}$ and $\sin ^{2} \theta_{23}=0.42$. The best fit prediction is broken down into the MINOS best fit spectrum (hatched pink) and MINOS+ best fit spectrum (hatched blue). . . . . . . . . . . .

7.5 MINOS and MINOS+ combined ratios of data and best fit spectra to the no oscillations prediction. The data ratio in black clearly shows muon neutrino disappearance. The green curve is the ratio for the prediction assuming the MINOS best fit from Ref. [64]. The ratio for the best fit at $\Delta m_{32}^{2}=2.41 \times 10^{-3} \mathrm{eV}^{2}$ and $\sin ^{2} \theta_{23}=0.42$ is drawn in blue. . . . . . . . . . . . . .

7.6 MINOS, MINOS+ combined contours in $\Delta m_{32}^{2}$ and $\sin ^{2} \theta_{23}$ compared to the MINOS contours [64]. The panel shows the $68 \%$ and $90 \%$ confidence limits on $\Delta m_{32}^{2}$ and $\sin ^{2} \theta_{23}$ for the normal mass (top) and the inverted mass hierarchy (bottom). The best fit from the full MINOS and MINOS+ exposure is plotted as a star. . . . . . . . . . . . . . . 
7.7 MINOS, MINOS+ combined $\Delta m_{32}^{2}$ and $\sin ^{2} \theta_{23}$ confidence limits. The left panel shows the $68 \%$ and $90 \%$ confidence limits on $\Delta m_{32}^{2}$ and $\sin ^{2} \theta_{23}$ for the normal mass hierarchy (top) and the inverted mass hierarchy (bottom). The best fit from the full MINOS+ beam exposure is plotted as a star. The right panels show the 1D likelihood profiles as functions of $\Delta m_{32}^{2}$ and $\sin ^{2} \theta_{23}$ for each mass hierarchy. . . . . . . . . . . . . . . .

7.8 MINOS, MINOS+ combined $\delta_{\mathrm{CP}}$ profiles for each mass hierarchy and $\theta_{23}$ octant. . . . . . . . . . . . . . .

7.9 Reproduced $\Delta m_{32}^{2}$ and $\sin ^{2} \theta_{23}$ contours from NOvA's fit to muon neutrino disappearance $[127,128] \ldots$. . . . . . . .

7.10 Initial $\Delta m_{32}^{2}$ and $\sin ^{2} \theta_{23}$ contours from combining MINOS, MINOS+, and NOvA fit surfaces. . . . . . . . . . . . . .

8.1 MINOS, MINOS $+\Delta m_{32}^{2}-\sin ^{2} \theta_{23}$ contours compared to other

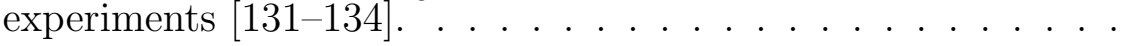

A.1 Runs 12 and 13 combined contained-vertex $v_{\mu}$ and $\bar{v}_{\mu}$ data spectrum and predictions at the far detector. The data spectrum is shown as black points. The orange curve is the predicted spectrum with no oscillations, and the best fit spectrum is shown in blue. The predicted spectrum assuming the best fit measured using the full MINOS data set [64] is shown in green. . . . . .

A.2 Runs 12 and 13 combined ratios of data and best fit spectra to the no oscillations prediction. The data ratio in black clearly shows muon neutrino disappearance. The green curve is the ratio for the prediction assuming the MINOS best fit from Ref. [64]. The ratio for the best fit is drawn in blue. . . . . . . .

A.3 Contours in $\Delta m_{32}^{2}$ and $\sin ^{2} \theta_{23}$ with $\Delta m_{32}^{2}$ from the fit to MINOS+ Runs 12 and 13 combined. The panel shows 68\% (orange) and $90 \%$ (blue) confidence limits on $\Delta m_{32}^{2}$ and $\sin ^{2} \theta_{23}$ for the normal mass (top) and the inverted mass hierarchy (bottom). The best fit is plotted as a star. . . . . . . . . . .

A.4 MINOS+ Runs 12 and $13 \Delta m_{32}^{2}$ and $\sin ^{2} \theta_{23}$ confidence limits. The left panel shows the $68 \%$ and $90 \%$ confidence limits on $\Delta m_{32}^{2}$ and $\sin ^{2} \theta_{23}$ for the normal mass hierarchy (top) and the inverted mass hierarchy (bottom). The best fit from the full MINOS+ beam exposure is plotted as a star. The right panels show the 1D likelihood profiles as functions of $\Delta m_{32}^{2}$ and $\sin ^{2} \theta_{23}$ for each mass hierarchy. . . . . . . . . . . . . 
B.1 MINOS+ contours in $\Delta m_{32}^{2}$ vs $\sin ^{2} \theta_{23}$. The $68 \%$ confidence limit is in orange, and the $90 \%$ confidence limit is in blue. The best fit from the full MINOS+ beam exposure, shown as the black star, lies in the inverted mass hierarchy and upper octant of $\theta_{23} .161$

C.1 MINOS and MINOS+ combined contained-vertex $\nu_{\mu}$ and $\bar{v}_{\mu}$ data spectrum and predictions at the far detector. The data spectrum is shown as black points. The orange curve is the predicted spectrum with no oscillations, and the blue curve is the best fit to the data for $\Delta m_{32}^{2}=2.41 \times 10^{-3} \mathrm{eV}^{2}$ and $\sin ^{2} \theta_{23}=0.42$. The predicted spectrum assuming the best fit measured using the full MINOS data set [64] is shown in green. 165

C.2 MINOS and MINOS+ contours in $\Delta m_{32}^{2}$ and $\sin ^{2} \theta_{23}$. The $68 \%$ confidence limit is in orange, and the $90 \%$ confidence limit is in blue. The best fit from the full MINOS and MINOS+ neutrino exposure, shown as the black star, lies in the normal mass hierarchy and lower octant of $\theta_{23} \ldots \ldots \ldots \ldots$ 


\section{Chapter 1}

\section{Neutrino Oscillations}

In the Standard Model (SM) of particle physics, neutrinos are massless and electrically neutral leptons with spin $1 / 2$ that only interact through the weak force. Although these properties make neutrinos challenging to detect, there is a global effort to study these particles. The observation of neutrino oscillations implies that neutrinos are massive and provided evidence of new physical phenomena. The field of neutrino oscillation physics offers answers to questions about neutrino mass and its implications.

\section{$1.1 \quad$ Neutrinos}

Measurements show there are three types or flavors of neutrinos, one for each charge lepton: electron $\left(v_{\mathrm{e}}\right)[1,2]$, muon $\left(v_{\mu}\right)[3]$, and tau $\left(v_{\tau}\right)[4]$. These three flavors are assumed to be massless by the SM.

An upper limit on the number of neutrino flavors was measured at particle colliders by studying the $\mathrm{Z}$ boson. Figure 1.1 shows the $\mathrm{Z}$ resonance measured by the Large Electron-Positron collider (LEP) experiments which agrees best with the $\mathrm{Z}$ line-shape prediction when the number of neutrinos is 3. 


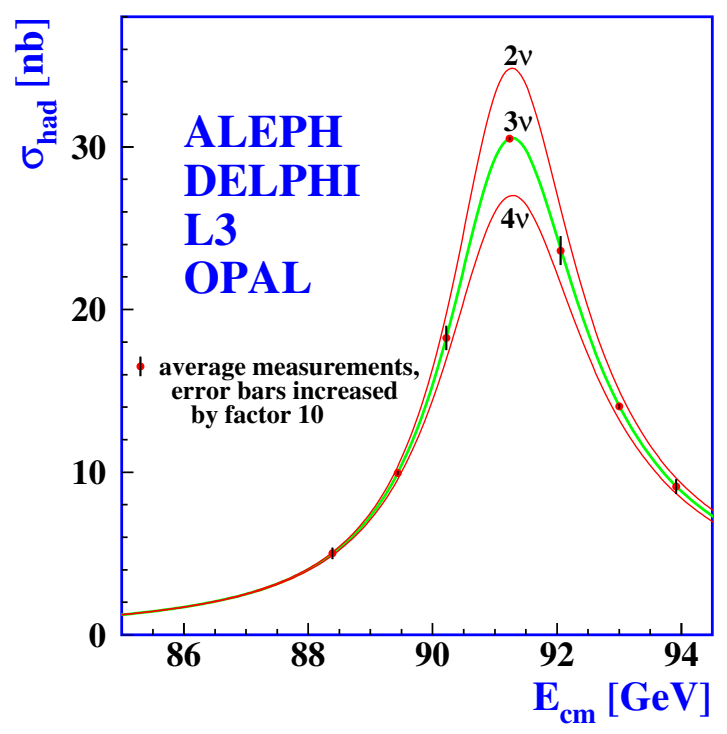

Figure 1.1: Measurements of the hadron production cross section around the $\mathrm{Z}$ resonance by the LEP experiments [5]. The curves are the predicted cross sections for two, three, and four neutrinos with Standard Model couplings and negligible mass.

Strict limits on the number of neutrino flavors come from carefully studying Z boson decay. Measurements from the SLAC Linear Collider (SLC) and CERN's LEP experiments constrain the number of SM neutrinos, also called active neutrinos, with mass less than $m_{\mathrm{Z}} / 2$ to be $N_{v}=2.9840 \pm 0.0082$ [5] using the $\mathrm{Z}$ boson decay width.

Additional limits on the number of neutrinos come from cosmology since massive neutrinos contribute to the energy density of the universe and formation of the early universe. These effects appear as anisotropies in the Cosmic Microwave Background (CMB), which constrain the number of relativistic neutral particle species in the early universe, $N_{\text {eff }}$, as well as the sum of 
neutrino masses. Only considering the SM particles, the value of $N_{\text {eff }}$ would be 3 for the number of neutrinos. However, when neutrino interactions and other processes are considered the value of $N_{\text {eff }}$ is modified. The calculated value including these processes is $N_{\text {eff }}=3.045$ [6]. Data from the Planck satellite observations of the CMB combined with baryon acoustic oscillation measurements find $N_{\text {eff }}=2.99 \pm 0.17[7]$, consistent with the SM, and constrain the sum of the neutrino masses to $\sum m_{v}<0.12 \mathrm{eV}$.

Neutrinos interact only through the weak force. Neutrinos participate in charged-current (CC) interactions where a virtual $\mathrm{W}$ boson is exchanged and neutral-current (NC) interactions where a virtual $\mathrm{Z}$ boson is exchange. Both $\mathrm{CC}$ and $\mathrm{NC}$ vertices involving neutrinos are drawn in Fig. 1.2. In a detector, a CC neutrino interaction is identified by a charged lepton traveling through the detector. A neutral current interaction, on the other hand, results in deposits of energy either from causing the target particle of the interaction to recoil or break apart.

\subsection{Evidence for Neutrino Oscillations}

In 1968, Ray Davis and colleagues [9] observed a significant deficit in the solar $v_{\mathrm{e}}$ flux relative to the predictions from J. Bahcall, N. Bahcall, and Shaviv [10]. This was known as the solar neutrino problem. It took 25 years before this

difference between the measured solar flux and predictions from the standard solar model (SSM) was resolved. The Sudbury Neutrino Observatory (SNO) experiment measured both $v_{\mathrm{e}} \mathrm{CC}$ and $\mathrm{NC}$ solar neutrino-induced interactions. 

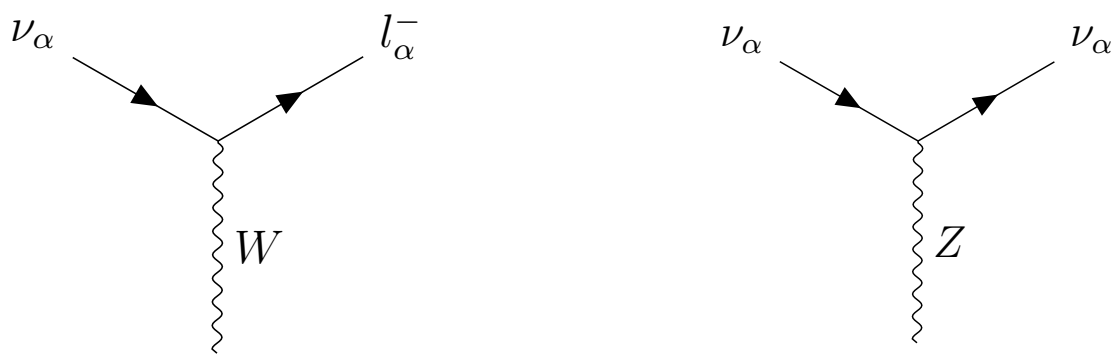

Figure 1.2: Diagrams of charged-current (left) and neutral-current (right) vertices involving neutrinos of flavor $\alpha, \nu_{\alpha}$, and corresponding charged lepton, $l_{\alpha}^{-}$. These diagrams were made using TikZ-Feynman [8].

The NC channel provided sensitivity to the other neutrino flavors, and together the $\mathrm{NC}$ and $v_{\mathrm{e}}$-CC flux agreed with the SSM $[11,12]$. While the measurement confirmed that the total solar neutrino flux agreed with the SSM, it implied that electron neutrinos produced by the sun were transforming in flavor.

At the same time as there were discrepancies between the predicted and measured solar neutrino flux, Kamiokande-II reported what would be known as the atmospheric neutrino anomaly [13]. When primary cosmic rays hit the atmosphere they produce many pions. These pions decay to neutrinos,

$$
\pi^{+} \rightarrow \mu^{+}+v_{\mu}, \quad \pi^{-} \rightarrow \mu^{-}+\bar{v}_{\mu}
$$

The muons in Eq. 1.1 can decay before reaching the ground resulting in

$$
\mu^{+} \rightarrow \mathrm{e}^{+}+v_{\mathrm{e}}+\bar{v}_{\mu}, \quad \mu^{-} \rightarrow \mathrm{e}^{-}+\bar{v}_{\mathrm{e}}+v_{\mu}
$$

The neutrinos produced in the atmosphere through the processes in Eqs. 1.1 
and 1.2 are called atmospheric neutrinos. Using Eqs. 1.1 and 1.2 the ratio of the $v_{\mu}+\bar{v}_{\mu}$ flux to $v_{\mathrm{e}}+\bar{v}_{\mathrm{e}}$ flux can be estimated as

$$
\frac{\phi_{v_{\mu}}+\phi_{\bar{v}_{\mu}}}{\phi_{v_{\mathrm{e}}}+\phi_{\bar{v}_{\mathrm{e}}}} \approx 2 .
$$

Instead, Kamiokande-II measured a flux ratio $\approx 1$. In 1998, the SuperKamiokande (SK) experiment announced that they had observed a zenith angle dependence for the $v_{\mu}$ atmospheric neutrino flux $[14,15]$. Fewer muon neutrinos were coming from the far side of the Earth than from above the detector. Somehow, muon neutrinos traveling through the Earth were disappearing.

The observations from SK and SNO could both be explained by neutrinos changing their flavors, a phenomenon known as neutrino oscillations.

\subsection{Neutrino Mixing and Oscillations}

The theory of neutrino mixing and oscillations was developed by Pontecorvo, Maki, Nakagawa, and Sakata [16-18]. Experimental data on neutrinos from the sun, the atmosphere, reactors, and accelerators can all be explained by three-flavor mixing of massive neutrinos. The fact that neutrinos oscillate implies that at least one must have non-zero mass. This contradiction with the SM is a hint of new physics, especially given that the neutrino mass is known to be substantially smaller than the other fermions. 


\subsubsection{Neutrino Mixing}

Neutrino mixing describes the superposition of neutrino mass eigenstates within neutrino flavor eigenstates:

$$
\left|v_{\alpha}\right\rangle=\sum_{i} U_{\alpha i}^{*}\left|v_{i}\right\rangle
$$

with $\alpha=\mathrm{e}, \mu^{-}, \tau$ and $i=1,2,3$ labeling the flavor and mass eigenstates, respectively. In Eq. 1.4, $U$ is the Pontecorvo-Maki-Nakagawa-Sakara (PMNS) mixing matrix. In the three-flavor model, this matrix can be parameterized as,

$$
U=\left(\begin{array}{ccc}
c_{12} c_{13} & s_{12} c_{13} & s_{13} e^{-i \delta_{\mathrm{CP}}} \\
-s_{12} c_{23}-c_{12} s_{23} s_{13} e^{i \delta_{\mathrm{CP}}} & c_{12} c_{23}-s_{12} s_{23} s_{13} e^{i \delta_{\mathrm{CP}}} & s_{23} c_{13} \\
s_{12} s_{23}-c_{12} c_{23} s_{13} e^{i \delta_{\mathrm{CP}}} & -c_{12} s_{23}-s_{12} c_{23} s_{13} e^{i \delta_{\mathrm{CP}}} & c_{23} c_{13}
\end{array}\right),
$$

where $c_{i j}$ and $s_{i j}$ denote $\cos \theta_{i j}$ and $\sin \theta_{i j}$. Thus three-flavor mixing is parameterized at a minimum by three mixing angles $\left(\theta_{12}, \theta_{13}, \theta_{23}\right)$ and a Charge-Parity (CP) violation $(\mathrm{CPV})$ phase, $\delta_{\mathrm{CP}}$. The form in Eq. 1.5 is sufficient for the study of three-flavor neutrino oscillations; however, it assumes that neutrinos are Dirac in nature, and the neutrino is distinguishable from its antiparticle. Since neutrinos are neutral, they are allowed to be their own antiparticle. In this case, neutrinos would be described as Majorana particles, and two CPV phases need to be added to fully describe the mixing. This addition can be achieved by multiplying Eq. 1.5 by a diagonal matrix with the CPV phases of the form,

$$
M=\operatorname{diag}\left(1, e^{i \phi_{1}}, e^{i \phi_{2}}\right)
$$

The Majorana neutrino mixing matrix is then formed by the product $U M$. 


\subsection{Neutrino Oscillations Formalism}

Neutrino oscillations are a consequence of neutrino mixing. This treatment of neutrino oscillations closely follows the derivations in Ref. [19]. Neutrinos are created in flavor states, but their propagation depends on their mass. The neutrino mass states are eigenstates of the Hamiltonian with energy eigenvalues $E_{k}$,

$$
H\left|v_{k}\right\rangle=E_{k}\left|v_{k}\right\rangle
$$

Using the Schrödinger equation, the time evolution of a flavor eigenstate in vacuum is written

$$
\left|v_{\alpha}(t)\right\rangle=\sum_{k} U_{\alpha k}^{*} e^{-i E_{k} t}\left|v_{k}\right\rangle
$$

Since $U$ is a unitary matrix, the mass states can be expressed in terms of mixing of the flavor states,

$$
\left|v_{k}\right\rangle=\sum_{\alpha} U_{\alpha k}\left|v_{\alpha}\right\rangle
$$

Using Eq. 1.9, the time evolution of a flavor state can be expressed as

$$
\left|v_{\alpha}(t)\right\rangle=\sum_{\beta}\left(\sum_{k} U_{\alpha k}^{*} e^{-i E_{k} t} U_{\beta k}\right)\left|v_{\beta}\right\rangle .
$$

The probability of finding $\left|v_{\alpha}\right\rangle$ in the state $\left|v_{\beta}\right\rangle$ after time, $t$, is given by

$$
\begin{aligned}
P_{v_{\alpha} \rightarrow v_{\beta}} & =\left|\left\langle v_{\beta} \mid v_{\alpha}(t)\right\rangle\right|^{2} \\
& =\sum_{k, l} U_{\alpha k}^{*} U_{\beta k} U_{\alpha l} U_{\beta l}^{*} e^{-i\left(E_{k}-E_{l}\right) t}
\end{aligned}
$$


The energy eigenvalues are given by

$$
E_{k}=\sqrt{\vec{p}^{2}+m_{k}^{2}}
$$

In the ultrarelativistic limit, Eq. 1.12 can be approximated by

$$
E_{k} \approx E+\frac{m_{k}^{2}}{2 E}
$$

Using Eq. 1.13,

$$
E_{k}-E_{l} \approx \frac{\Delta m_{k l}^{2}}{2 E}
$$

where $\Delta m_{k l}^{2}$ is the mass squared difference

$$
\Delta m_{k l}^{2} \equiv m_{k}^{2}-m_{l}^{2}
$$

and $E$ is the the neutrino energy neglecting the mass. Then taking time to be equivalent to distance, $L$, the neutrino oscillation probability becomes

$$
P_{v_{\alpha} \rightarrow v_{\beta}}=\sum_{k, l} U_{\alpha k}^{*} U_{\beta k} U_{\alpha l} U_{\beta l}^{*} \exp \left(\frac{-i \Delta m_{k l}^{2} L}{2 E}\right) .
$$

The oscillation probability does not depend on Majorana CPV phases and is therefore insensitive to whether the neutrinos are Dirac or Majorana particles [19].

In the case of three-flavor oscillations, also called standard oscillations, the neutrino oscillation probabilities depend on 6 parameters: 3 mixing angles $\left(\theta_{12}, \theta_{13}, \theta_{23}\right), 1 \mathrm{CPV}$ phase $\left(\delta_{\mathrm{CP}}\right)$, and two mass squared differences $\left(\Delta m_{21}^{2}\right.$, $\left.\Delta m_{32}^{2}\right)$.

When active neutrinos propagate through matter they experience an effective potential due to their interactions. For three-flavor oscillations the 
effective potential is only relevant for electron neutrinos and antineutrinos due to differences in their $\mathrm{CC}$ interactions with electrons in matter. Thus, in matter, the vacuum Hamiltonian, $H_{0}$ needs to be modified by an effective potential,

$$
V_{\mathrm{CC}}=\left(\begin{array}{ccc} 
\pm \sqrt{2} G_{\mathrm{F}} N_{\mathrm{e}} & 0 & 0 \\
0 & 0 & 0 \\
0 & 0 & 0
\end{array}\right) .
$$

Then the matter Hamiltonian in the flavor basis can be expressed as

$$
H_{\text {matter }}^{\mathrm{F}}=U H_{0}^{\mathrm{M}} U^{\dagger}+V_{\mathrm{CC}} .
$$

Due to this effective potential, it is possible for a resonance to occur that can result in total transition from one flavor to another if the resonance region is large enough. This resonance effect is known as the MSW effect after Mikheev, Smirnov, [20,21] and Wolfenstein [22]. The MSW effect is particularly important for the treatment of solar neutrinos which can experience these resonant transitions as a result of their production energy and the electron density of the sun.

\subsection{Measuring Three-Flavor Oscillation Parameters}

Neutrino oscillation experiments perform appearance measurements, which measure the transition probability from one flavor to another, or disappearance measurements, which measure the survival probability for a par-

ticular flavor. Depending on the neutrino source experiments employ, they are able to control the source-to-detector distance, or baseline, and the neu- 
trino energy. Neutrino oscillation experiments select the baseline and neutrino energy to be sensitive to different oscillation parameters.

Solar neutrinos and nuclear reactors are used to measure $\Delta m_{21}^{2}$ and $\theta_{12}$ using $v_{\mathrm{e}}$ and $\bar{v}_{\mathrm{e}}$ disappearance. These oscillation parameters are sometimes referred to as the solar mixing parameters. Reactor experiments sensitive to these parameters have a baseline on the order of $10^{2} \mathrm{~km}[19]$.

Reactor experiments with baselines on the order of $1 \mathrm{~km}[19]$ use $\bar{v}_{\mathrm{e}^{-}}$ disappearance to measure $\theta_{13}$.

Muon neutrinos produced using accelerators can also be used to measure $\theta_{13}$ by studying $v_{\mathrm{e}}$ appearance over long baselines on the order of $10^{3} \mathrm{~km}$ [19]. These long-baseline oscillation experiments measure $\Delta m_{32}^{2}$ and $\theta_{23}$ along with experiments that measure atmospheric neutrino experiments studying $v_{\mu}$ disappearance. For this reason, $\Delta m_{32}^{2}$ and $\theta_{23}$ are sometimes called the atmospheric oscillation parameters.

Furthermore, reactor experiments have measured $\theta_{13}$ to high enough precision [23-25] such that long-baseline accelerator experiments may be able to measure $\delta_{\mathrm{CP}}$ with $v_{\mathrm{e}}$ appearance. Additionally, these appearance measurements are sensitive to the sign of $\Delta m_{32}^{2}$ and the octant of $\theta_{23}$, two important open question in neutrino oscillation physics. 


\subsection{Open Questions in Neutrino Oscillation Physics}

The present and future neutrino oscillation programs aim to illuminate the following unknown neutrino properties.

\section{Mass Hierarchy}

In this work, the mass hierarchy refers to the sign of $\Delta m_{32}^{2}$. If $\Delta m_{32}^{2}>0$, then there is the Normal Hierarchy (NH) with $m_{1}<m_{2}<m_{3}$. If $\Delta m_{32}^{2}>0$, then there is the Inverted Hierarchy (IH) with $m_{3}<m_{1}<m_{2}$. In some contexts the term hierarchy is reserved to describe the relative scale of the neutrino masses, and term ordering is preferred to describe the sign of $\Delta m_{32}^{2}$. The subtleties of these definitions are discussed in Ref. [26].

\section{Octant of $\theta_{23}$}

It is still undetermined whether or not $\theta_{23}$ is maximal $\left(\theta_{23}=\pi / 4\right)$. If $\theta_{23} \neq \pi / 4$, then it remains to determine whether $\theta_{23}$ is in the lower octant $\left(\theta_{23}<\pi / 4\right)$ or in the upper octant $\left(\theta_{23}>\pi / 4\right)$. Beyond oscillations, this value has implications for fundamental symmetries in particle physics [27-29].

\section{Value of $\delta_{\mathrm{CP}}$}

Whether or not neutrino oscillations violate $\mathrm{CP}$ is an important question since $\mathrm{CP}$ violation in neutrino oscillations might provide an explanation

of the baryon asymmetry of the Universe [30-32]. If $e^{i \delta_{\mathrm{CP}}}$ is real, then neutrino oscillations conserve CP. 


\subsection{Accelerator Neutrino Experiments}

Accelerator neutrino experiments have the ability to answer these open questions. These experiments are classified by their neutrino source, their baseline, and energy spectrum. Reference [33] provides a comprehensive review of accelerator-based neutrino beams.

Current accelerator-based neutrino oscillation experiments produce their neutrino beams via decay in flight of pions and kaons. The energy spectrum of the experiment is then controlled by focusing the mesons in a certain range of momenta and is on the order of GeVs.

These experiments are classified as short or long baseline. Short-baseline experiments have baseline distances ranging from on the order of $10 \mathrm{~m}$ to $1 \mathrm{~km}$ [19]. Long-baseline experiments on the other hand have baselines on the order of $10^{3} \mathrm{~km}$. In the context of neutrino oscillations, short-baseline experiments with $\mathrm{GeV}$ neutrino beams are useful to search for large mass squared differences that would be associated with sterile neutrinos.

The energy spectra of these beams are classified as wide band with a neutrino flux that covers a broad range of energies, or narrow band with a neutrino flux that samples a small range of energies.

First generation long-baseline oscillation experiments used wide-band beams, and their detectors were located on-axis in the center of the beam. The second generation of long-baseline oscillation experiments is a special class called off-axis experiments. These experiments measure a narrow-band 
spectrum at their off-axis position in a wide-band neutrino beam.

Fermilab operated the on-axis MINOS+ [34] and $14.6 \mathrm{mrad}$ off-axis NOvA [35] experiments simultaneously, and their simulated neutrino spectra are compared in Fig. 1.3.

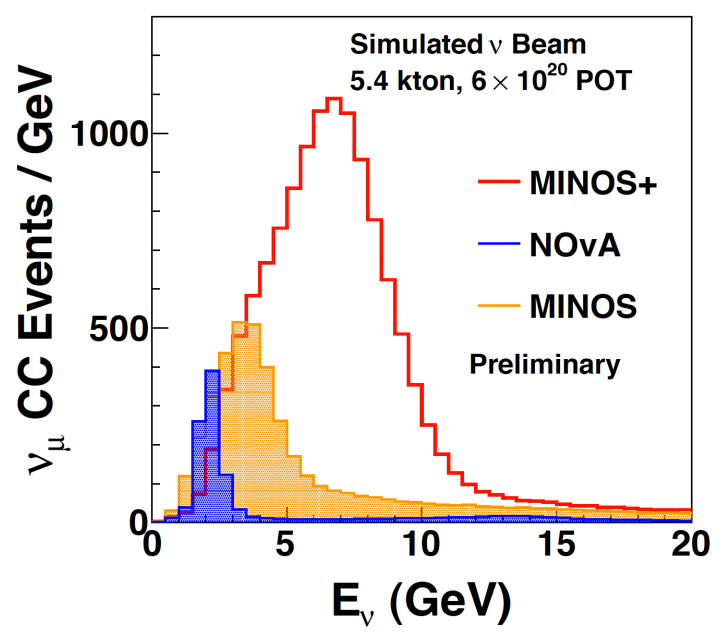

Figure 1.3: Neutrino spectrum on-axis for MINOS+ and off-axis for NOvA. The plot also shows the lower energy spectrum produced on-axis for the predecessor to MINOS+, MINOS. 


\section{Chapter 2}

\section{The MINOS+ Experiment}

The MINOS+ experiment used the MINOS detectors in the mediumenergy NuMI beam configuration provided for the new NOvA experiment to take data for three years. The MINOS Near Detector (ND) is located at Fermilab $1.04 \mathrm{~km}$ from the NuMI target and $100 \mathrm{~m}$ (225 mwe) underground. The MINOS Far Detector (FD) was located at the University of Minnesota's Soudan Underground Laboratory in Soudan, MN. The FD was $735 \mathrm{~km}$ from the NuMI target and $705 \mathrm{~m}$ underground (2070 mwe), $210 \mathrm{~m}$ below sea level. These detectors were functionally equivalent magnetized steel-scintillator, tracking and sampling calorimeters.

In the context of three-flavor neutrino oscillations, MINOS and MINOS+ have a unique data set with neutrinos and antineutrinos as well as statistics covering a large range of $L / E$ at and beyond the first oscillation maximum. This data set allows for a stringent test of the three-flavor neutrino oscillation model. The higher energy beam of MINOS+ tests the validity of the three-neutrino model beyond the first oscillation maximum and allows searches for exotic phenomena like sterile neutrinos [36], large extra di-

mensions [37], and non-standard interactions [38] by studying muon neutrino 

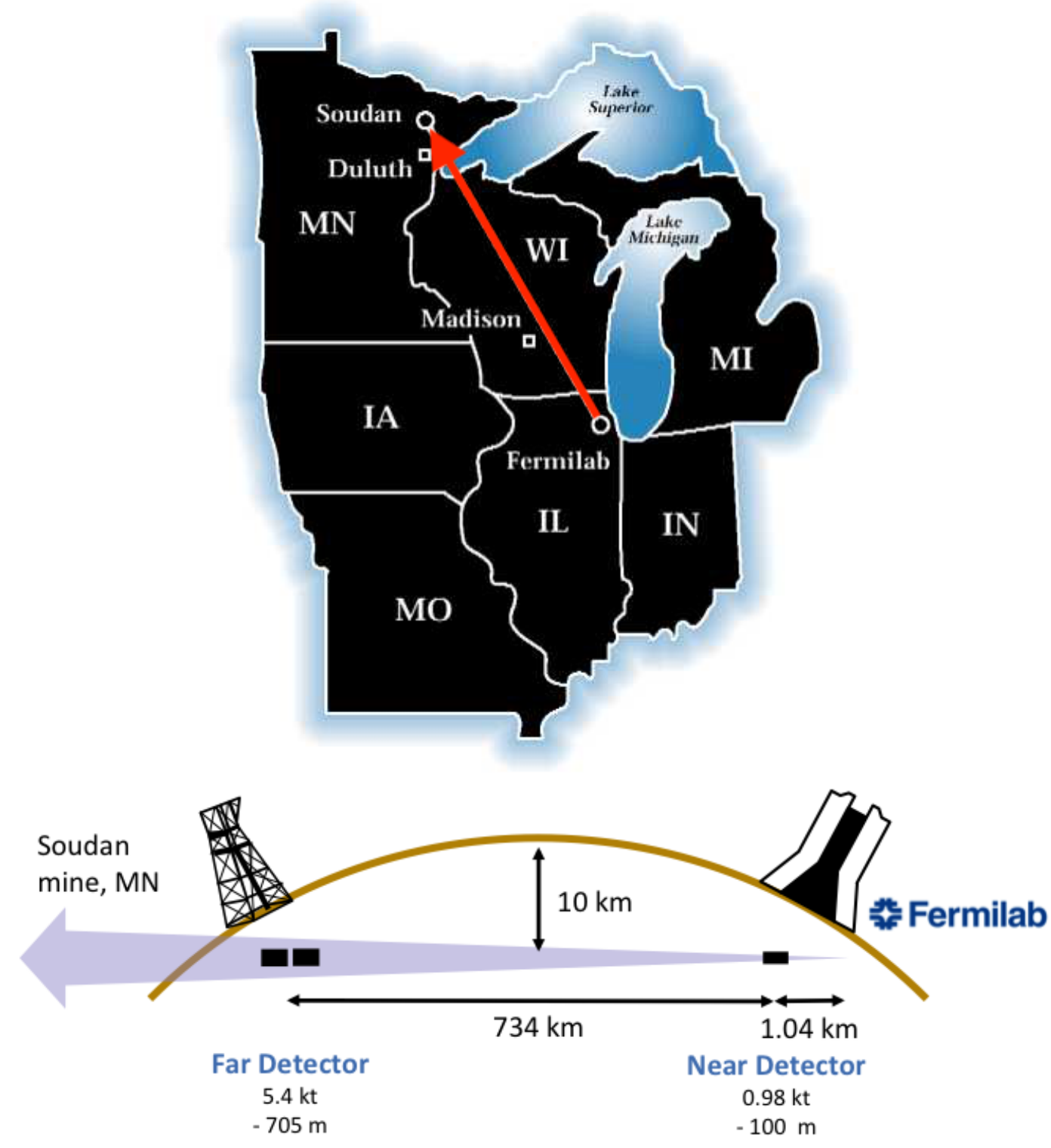

Figure 2.1: The MINOS and MINOS+ experimental setup. The map above illustrates the $735 \mathrm{~km}$ baseline from Fermilab to Soudan, MN where the MINOS far detector was located. The drawing on the bottom summarizes the masses, positions, and sizes relative to the neutrino beam of the MINOS detectors. 
disappearance and electron neutrino appearance.

\subsection{NuMI Neutrino Beam}

The NuMI neutrino beam is produced by colliding $120 \mathrm{GeV}$ protons from Fermilab's Main Injector [39] into a graphite target. The resulting pions and kaons are then focused by two magnetic horns into a decay pipe. The magnetic horns allow the beam to be operated in either a $v_{\mu}$-mode or $\bar{v}_{\mu^{-}}$ mode. The dominant decays that produce the neutrino are shown in Eq. 2.1.

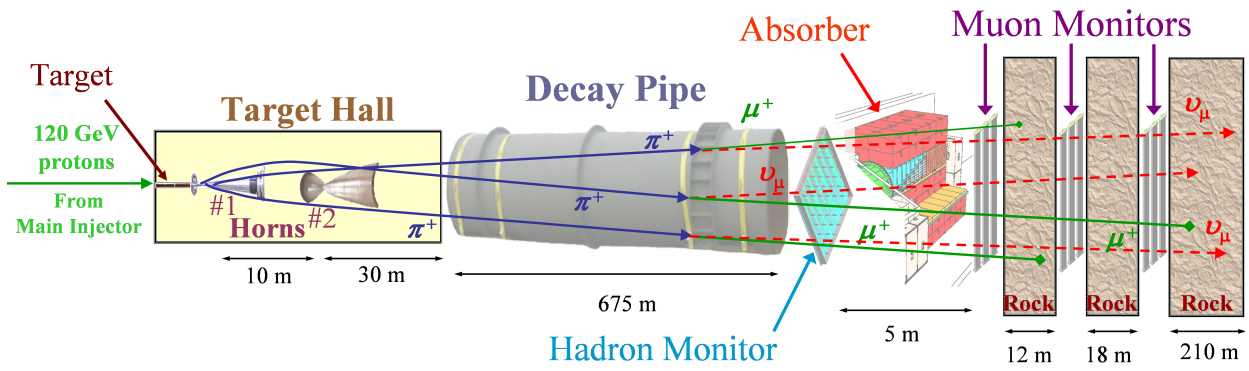

Figure 2.2: Cartoon of the NuMI neutrino beamline.

$$
\begin{aligned}
& \pi^{ \pm} \rightarrow \mu^{ \pm}+v_{\mu} / \bar{v}_{\mu} \\
& \mathrm{K}^{ \pm} \rightarrow \mu^{ \pm}+v_{\mu} / \bar{v}_{\mu} \\
& \mu^{ \pm} \rightarrow \mathrm{e}^{ \pm}+\bar{v}_{\mu} / \nu_{\mu}+v_{\mathrm{e}} / \bar{v}_{\mathrm{e}}
\end{aligned}
$$

There is a small contribution to neutrino flux from unfocused $\mathrm{K}_{\mathrm{L}}^{0}$ mesons that decay.

MINOS and MINOS+ collected 11 years of beam data from 2005 to 2016 using the MINOS detectors. MINOS collected primarily low-energy beam 
data from May 1, 2005 to April 29, 2012. MINOS+ collected medium-energy beam data from September 4, 2013 to June 29, 2016. MINOS+ operated in a medium-energy beam with a neutrino flux that peaked at $7 \mathrm{GeV}$. MINOS beam data were taken in two beam configurations, a low-energy configuration and a high-energy configuration. In total, MINOS ran in seven different beam configurations which were important for constraining the beam's neutrino flux $[40,41]$. The neutrino flux for the low-energy configuration peaked at $3 \mathrm{GeV}$, and the high-energy neutrino flux peaked at $9 \mathrm{GeV}$ [42]. MINOS+ benefited from an increase in beam intensity thanks to the Accelerator and NuMI Upgrades [35] and the Fermilab Proton Improvement Plan [43]. On June 13, 2016 the NuMI beam achieved a beam power of $700 \mathrm{~kW}$ making it the most powerful neutrino beamline worldwide. During MINOS operation the proton beam had an intensity of $2.2-3.6 \times 10^{13}$ protons per pulse $(\mathrm{ppp})$ and a beam power ranging from 270-345 kW [42]. MINOS+ operations saw $2.4-4.2 \times 10^{13} \mathrm{ppp}$ with a peak of $4.6 \times 10^{13} \mathrm{ppp}$. The typical MINOS+ proton beam power was $240-550 \mathrm{~kW}$ peaking at $600 \mathrm{~kW}^{1}$

Before protons coming from the main injector hit the graphite target, they pass through a protective component called a baffle. A diagram of the MINOS-era baffle is shown in Fig. 2.3. The baffle is a hollowed cylinder of graphite that protects the downstream NuMI components from being damaged by the high-intensity proton beam should it be mis-steered. The inner radius

\footnotetext{
${ }^{1}$ Although the NuMI beam did achieve proton beam power of $700 \mathrm{~kW}$ on June 13,2016 this was only a short test due to radiation risks. A collimator was installed during the 2016 shutdown to allow safe operation at proton beam powers of $700+\mathrm{kW}$ [44].
} 
of the baffle is set to 5 times the nominal proton beam width. This opening is approximately $5 \sigma$ of beam width, allowing the Gaussian beam to pass through the baffle. The NuMI beam for MINOS was designed to have a beam width of $1.1 \mathrm{~mm}$, and during operation the beam width varied from 1.1-1.2 $\mathrm{mm}$ [42]. The higher intensity proton beam for MINOS+ was designed to have a beam width of $1.3 \mathrm{~mm}$, and during operation the width varied from $1.1-1.3 \mathrm{~mm}$.

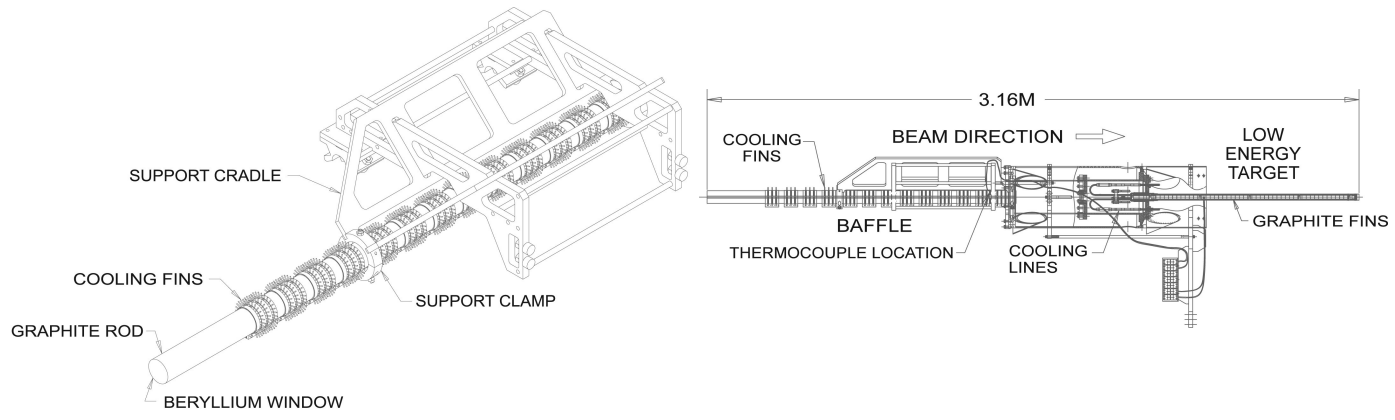

Figure 2.3: The MINOS era NuMI baffle. On the left is the baffle by itself. On the right is the baffle connected to the target module, when installed in the beamline the baffle and target are one unit. These drawing are from Ref. [42].

The protons collide with the NuMI target $68 \mathrm{~cm}$ after exiting the baffle. The mesons produced by the collision with the target are focused by the magnetic horns into a decay pipe $2 \mathrm{~m}$ in diameter and $675 \mathrm{~m}$ long [42]. At the downstream end of the decay pipe there is a hadron absorber to stop any hadrons that have survived thus far. Then there is $240 \mathrm{~m}$ of rock to stop any muons created by the beam from reaching the ND.

The alignment of the beamline components is important for the operation of the neutrino beam. The proton beam can scan across the target and be steered through the baffle around the target, an important capabil- 
ity for measuring component alignment $[45,46]$. In addition to being able to steer the proton beam, the baffle and target are mounted in a single module that can be remotely positioned. The upstream horn, Horn 1, can also be remotely positioned horizontally and vertically for better alignment. However, the downstream horn, Horn 2, is mounted in a fixed position based on the beam configuration. Figure 2.4 shows the positions of Horn 2 for low-energy, medium-energy, and high-energy configurations.

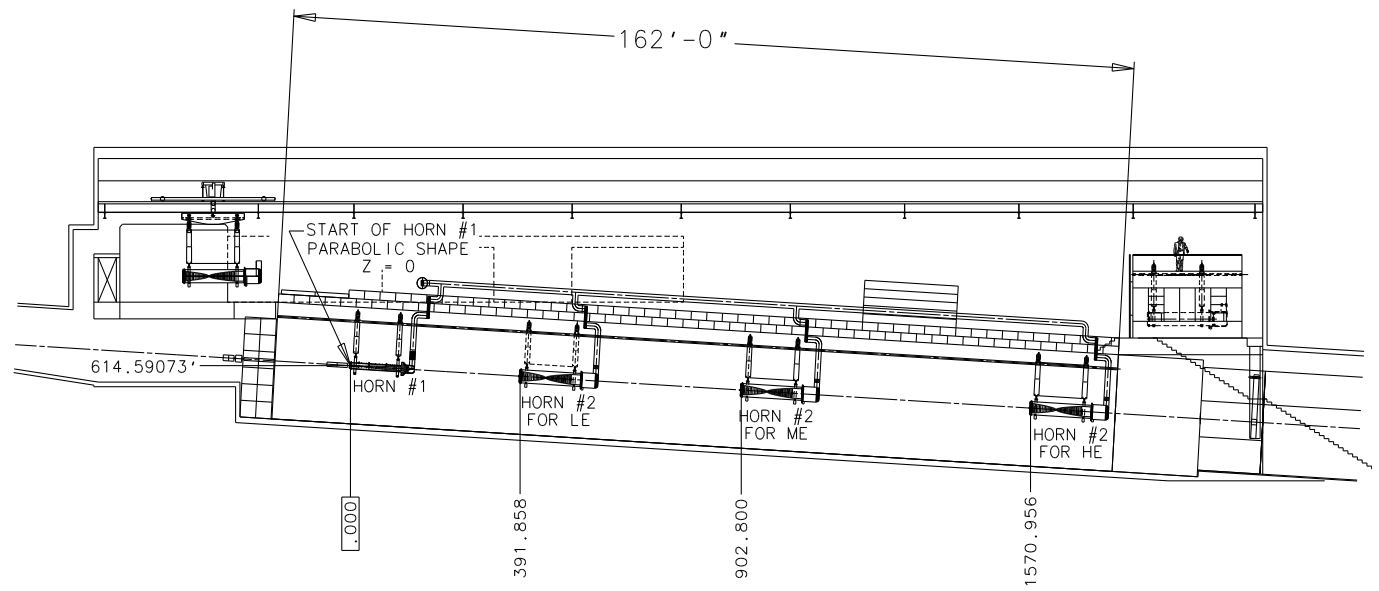

Figure 2.4: Drawing of the NuMI target hall showing Horn 2 at different positions for the low, medium, and high-energy beam configurations. The horn positions are labeled in inches. Drawing from Ref. [47].

The target is designed to optimize the desired neutrino spectrum while surviving operating conditions. In general, the neutrino beam energy is increased by increasing the distance between the target and Horn 1 and increasing the distance between Horn 1 and Horn 2. MINOS operated in a low-energy 
(LE) neutrino beam configuration which required part of the target to be inserted into Horn 1 (see Fig. 2.7). MINOS+ ran in a medium-energy (ME) configuration with the target located further upstream of Horn 1 compared to MINOS. Moving the target upstream has the effect of increasing the peak neutrino energy of the beam on-axis. For the ME configuration, Horn 2 was moved further downstream from Horn 1 relative to the LE configuration. The MINOS high-energy (HE) beam was achieved by only increasing the distance between the target and Horn 1. This HE configuration is sometimes referred to as pseudo-high-energy or semi-high-energy since Horn 2 was in its LE position. The ND spectra from the MINOS LE and HE configurations are compared to the MINOS+ ME configuration in Fig. 2.5.

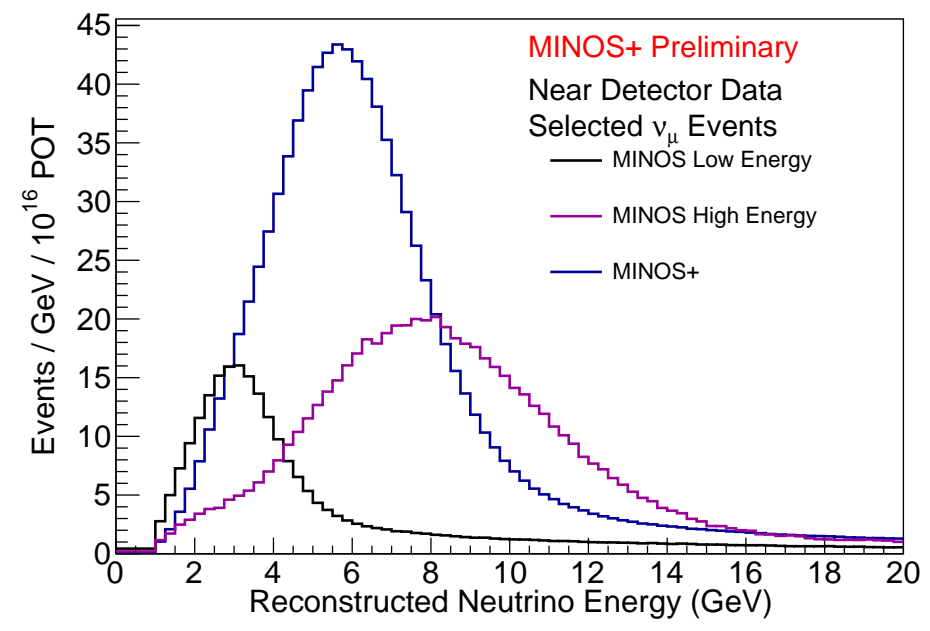

Figure 2.5: MINOS low-energy and high-energy $v_{\mu}$ CC spectra at the ND compared to the MINOS+ medium-energy $v_{\mu} \mathrm{CC}$ spectrum. 

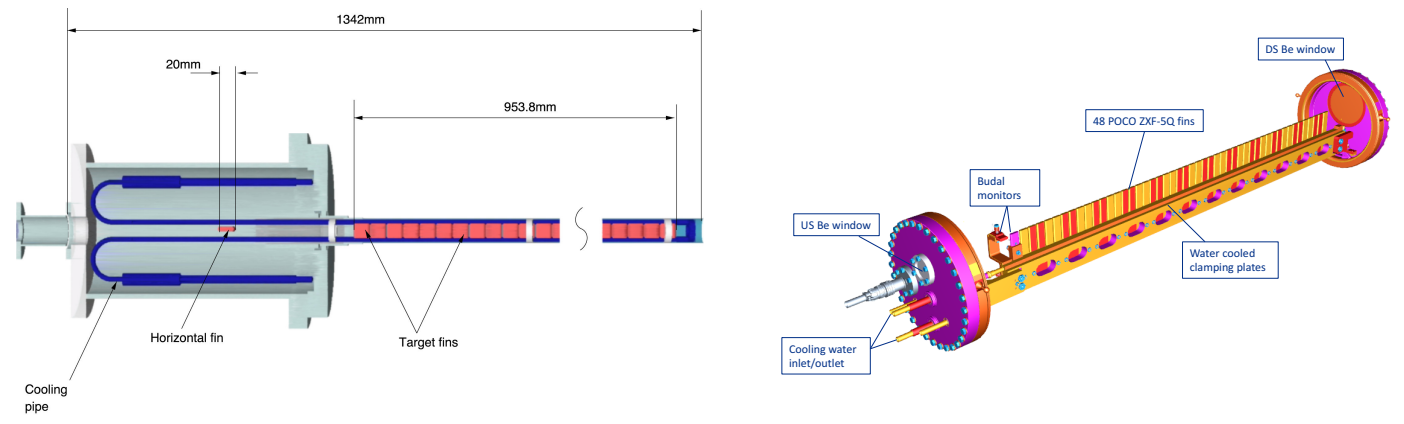

Figure 2.6: The NuMI low-energy and medium-energy targets. On the left is the target used by MINOS which was designed to be inserted into the upstream focusing horn. On the right is the target used by MINOS+ which was designed for a $700 \mathrm{~kW}$ proton beam. The diagrams adapted from Refs. [42, 48].

The nominal horn current during the MINOS low-energy configuration was $185 \mathrm{kA}$ and during the MINOS+ medium-energy configuration the nominal horn current was $200 \mathrm{kA}$. By changing the direction of the horn current, the NuMI beam operated in a neutrino or antineutrino mode. In neutrino mode, the current is flowing in the beam direction on the inner conductor of the horns, referred to as Forward Horn Current (FHC) and illustrated in Fig. 2.7. In antineutrino mode, the current is flowing opposite the beam direction on the inner conductor of the horns, referred to as Reverse Horn Current (RHC). MINOS collected FHC and RHC data while MINOS+ collected FHC data. The $v_{\mu}$-mode spectra for MINOS and MINOS+ at the ND are broken down to the $v_{\mu}$ and $\bar{v}_{\mu}$ components and compared in Fig. 2.8. The resulting CC beam spectrum from the MINOS+ beam at the ND is composed of $96.9 \% v_{\mu}$, $1.9 \% \bar{v}_{\mu}$, and $1.2 \% v_{\mathrm{e}}+\bar{v}_{\mathrm{e}}$. In comparison, the MINOS low-energy $v_{\mu}$-mode 
ND spectrum was composed of $92.9 \% v_{\mu}, 5.8 \% \bar{v}_{\mu}$, and $1.3 \% v_{\mathrm{e}}+\bar{v}_{\mathrm{e}}[40]$.

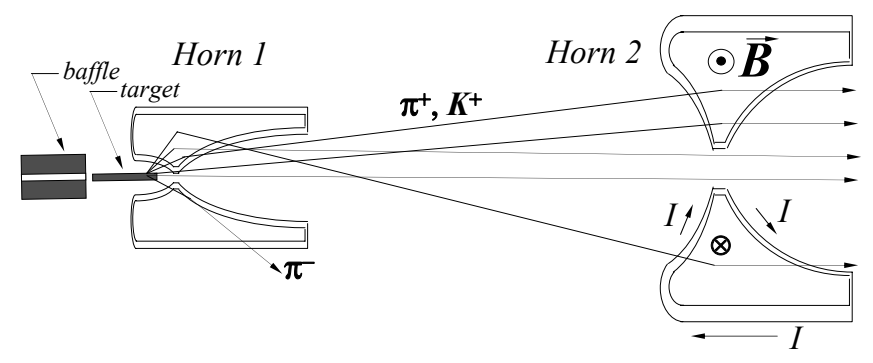

Figure 2.7: Cartoon of neutrino mode, Forward Horn Current, focusing of the mesons to produce the neutrino beam. In Forward Horn Current mode the horn current on the inner conductor of the horns flows in the same direction as the beam. Drawing from Ref. [40].
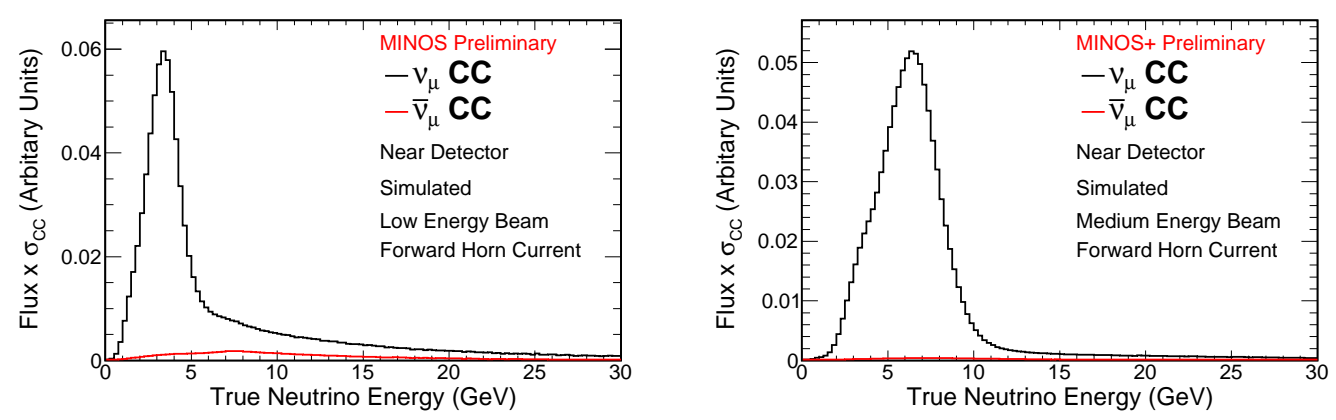

Figure 2.8: Comparison of $\boldsymbol{v}_{\mu}$ (black) and $\bar{v}_{\mu}$ (red) spectra for low-energy (left) and medium-energy (right) NuMI beam at the MINOS near detector. Figures from Ref. [49].

\subsection{The MINOS Detectors}

The MINOS+ experiment used the same detectors as the MINOS experiment but in a higher energy neutrino beam. The MINOS detectors [50] 
consisted of alternating planes of steel and scintillator. Each detector was magnetized by a current-carrying coil that ran parallel to the length of the detector. The magnetic field allowed the MINOS detectors to distinguish between $v_{\mu}$ and $\bar{v}_{\mu}$ charged current (CC) interactions based on the curvature of the resulting muon. The magnetic field of the detectors was set to complement the neutrino beam so that muons from the $v_{\mu}$-mode beam or antimuons from the $\bar{v}_{\mu}$-mode beam would be bent toward the center of the detectors.

Steel served as the target material of the detectors. For both detectors, the steel planes were $2.54 \mathrm{~cm}$ thick and positioned $5.95 \mathrm{~cm}$ apart as measured from center-to-center. The scintillator planes that made up the active volume of the detectors were mounted on the upstream side of the steel planes such that an active plane always was between two steel planes. The active planes were arranged in alternating views of $\pm 45^{\circ}$ relative to vertical, labeled $U$ and $V$, which prevented having strip connections at the bottom of the detector.

Each scintillator plane was constructed of extruded polystyrene strips $1 \mathrm{~cm}$ thick by $4.1 \mathrm{~cm}$ wide. A cutaway of a scintillator strip is shown in Fig. 2.9. The strips were co-extruded with a layer of polystyrene loaded with titanium dioxide, which provided a reflective coating to the inner walls of the strip. The light produced in each strip was collected via a wavelength shifting (WLS) fiber that was laid in the center of the wide face and ran the length of the strip. Each WLS fiber was coupled to a clear fiber which was read out by a pixel of a multi-anode photomultiplier tube (PMT) as drawn in Fig. 2.10.

$$
\text { MINOS }+v_{\mu} \text { CC interactions produce muons with energies of sev- }
$$




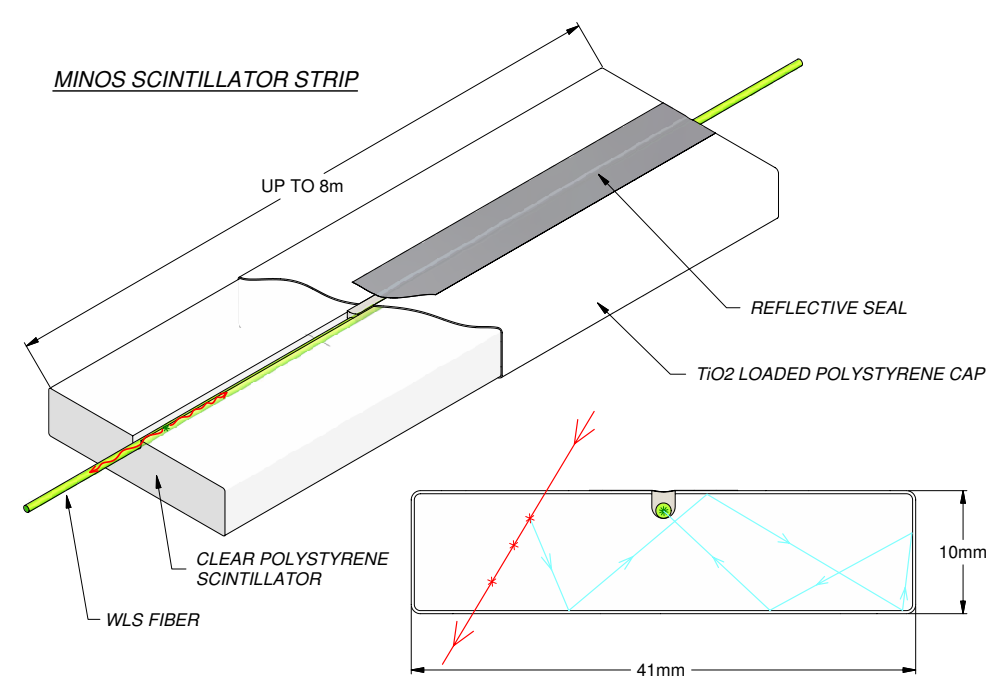

Figure 2.9: Cutaway drawing of a MINOS detector scintillator strip. Light produced by an ionizing particle is multiply reflected by the reflective titaniumdioxide coating. Light absorbed by the wavelength shifting fiber is re-emitted isotropically. Wavelength shifted photons whose directions fall within the total internal reflection cones are transported along the fiber to the edge of the detector in order to be readout. Drawing from Ref. [50] and made by M. Proga.

eral GeV. Taking into account the density of the MINOS detector steel, a minimally ionizing muon looses about $30 \mathrm{MeV}$ per detector plane. Thus a $3 \mathrm{GeV}$ muon from a neutrino interaction would traverse approximately 100 detector planes.

\subsubsection{Far Detector}

The 5.4 kt FD, Fig. 2.11, was made up of 486 regular octagonal steel planes $8 \mathrm{~m}$ edge to edge. The planes were grouped into two supermodules separated by a $1.15 \mathrm{~m}$ gap [50]. The upstream supermodule contained 249 


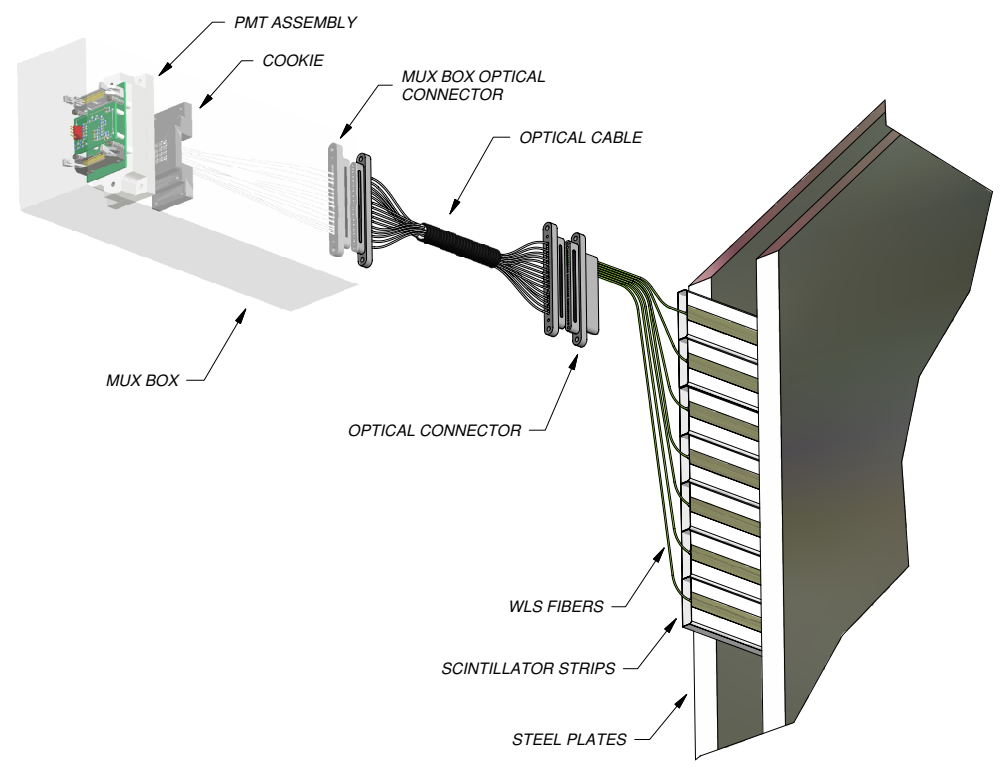

Figure 2.10: Schematic drawing of the readout of a scintillator module. An edge of a detector plane is on right side of the sketch is exaggerated to show how the scintillator strips are readout. The wavelength shifting fibers from the strips are coupled to clear optical fibers which carry the light to a pixel of the photomultiplier tube. Drawing from Ref. [50] and made by M. Proga.

planes and was $14.78 \mathrm{~m}$ long, and the downstream supermodule had 237 planes and was $14.10 \mathrm{~m}$ long. Each supermodule had its own magnetic coil that axially extended the length of the supermodule. In the fiducial volume, the FD had an average magnetic field of $1.42 \mathrm{~T}$. Each steel plane was assembled from eight, $2 \mathrm{~m}$ wide, $1.27 \mathrm{~cm}$ thick plates and then plug-welded together. The seams of the plates are apparent in the magnetic field map of an FD plane in Fig. 2.12.

Figure 2.13 shows an illustration of the two types of scintillator planes 

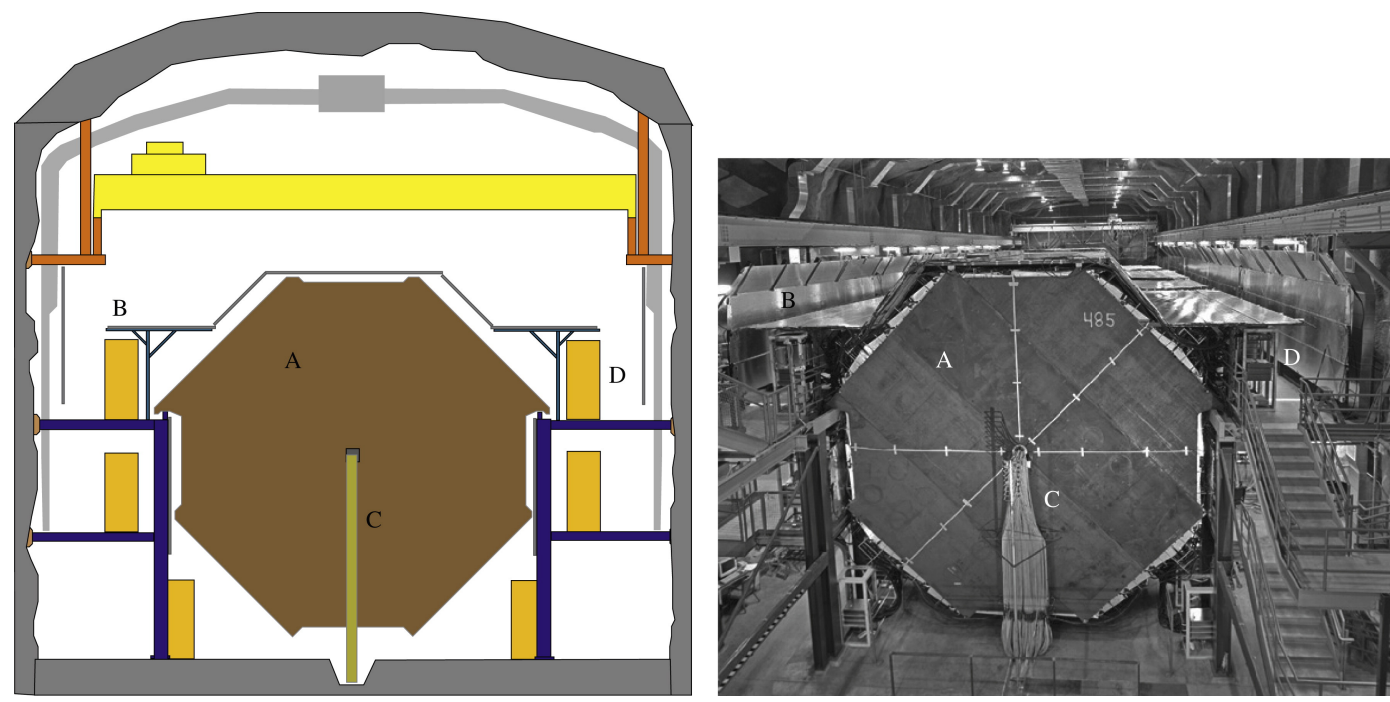

Figure 2.11: The MINOS far detector. On the left is a labeled drawing of the detector pictured on the right. This perspective is looking south toward Fermilab from the end of the second supermodule. "A" is the furthest downstream steel plane of the detector. "B" is part of the cosmic ray veto shield which is both above and along the sides of the detector. "C" is the end of the magnet coil. "D" is an electronics rack on one of the elevated walkways alongside the detector. This figure is from Ref. [50].

covering steel planes. The first active plane in each supermodule was a $V$ view. At the FD, the scintillator strips were readout at both ends with 8 non-adjacent strips mapped to a single pixel of the 16-pixel Hamamatsu M16 PMTs $[51,52]$. The FD was instrumented with a cosmic ray veto shield that covered the top of both supermodules and flanked the sides. The parts of the veto shield covering the top of the detector as well as the west and east walls of the cavern are visible in Fig. 2.11. The veto shield also partially covers the vertical faces on either side of the detector [50]. 

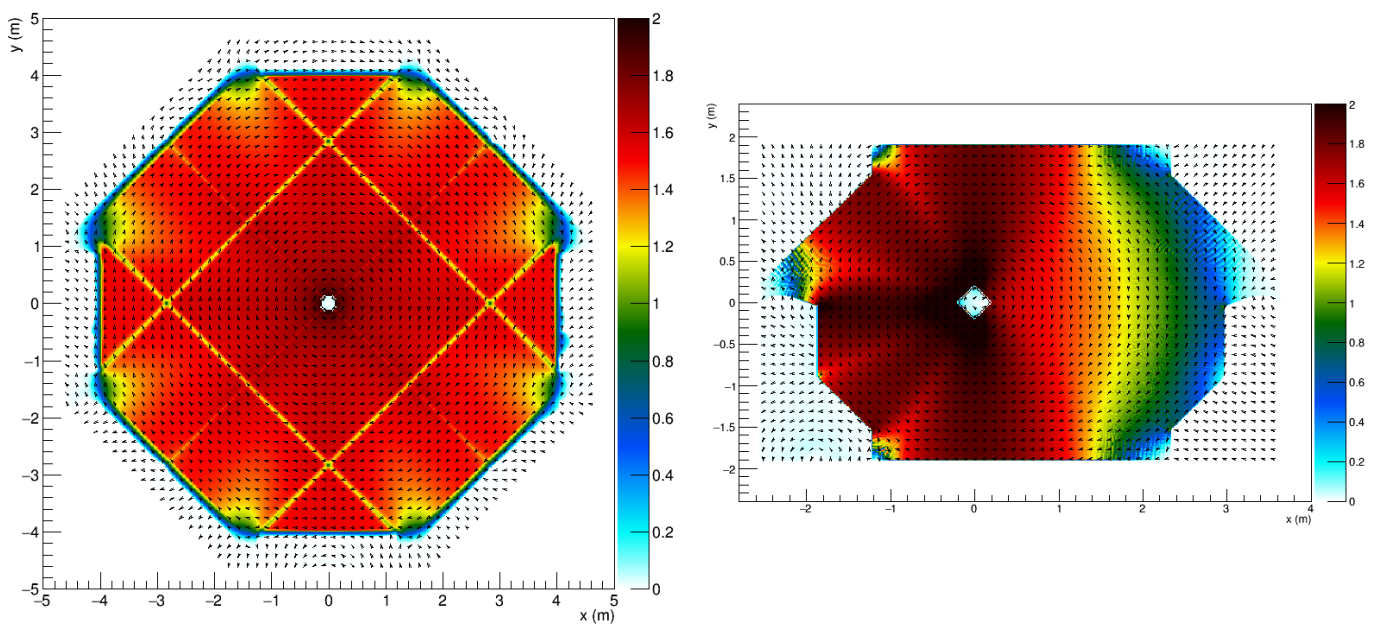

Figure 2.12: The magnetic field in the far detector (left) and near detector (right) courtesy of Robert Hatcher. These are from the perspective of looking toward the NuMI target. The $z$-axis of the histograms are in units of T. The features seen in the far detector plane are from the seams of the steel plates that were assembled to form a single far detector plane.
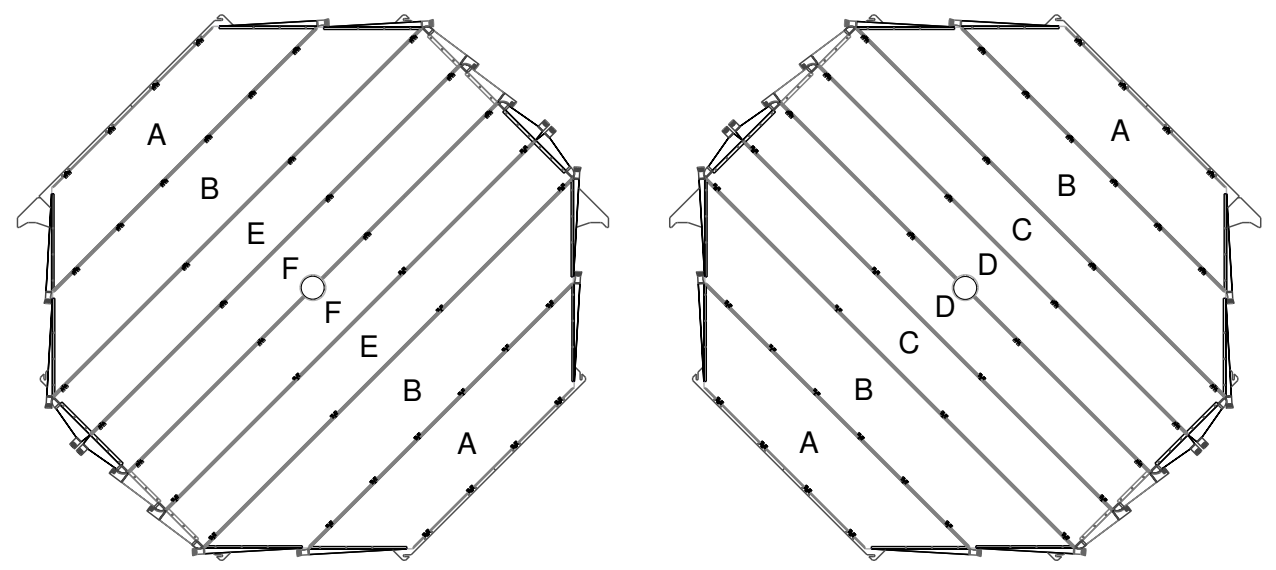

Figure 2.13: Layout of $U$ (left) and $V$ (right) modules on far detector planes. $U$ - and $V$-type planes were interleaved. "A" and "B" module types have 28 scintillator strips and the other types have 20 strips. The first (upstream) scintillator plane of each supermodule was of the $V$-type. This figure is from Ref. [50]. 


\subsubsection{Near Detector}

The MINOS ND shown in Fig. 2.14 is $0.98 \mathrm{kt}$ and consists of 282 steel squashed hexagonal planes, $3.8 \mathrm{~m}$ high and $4.8 \mathrm{~m}$ wide [53]. The steel plates are one single piece unlike the FD. The ND is arranged in two sections labeled the calorimeter and the spectrometer. The calorimeter is the upstream section and contains the fiducial volume of the detector. The fiducial volume is defined as a cylinder west of the ND coil hole that encompasses the beam center. In the fiducial volume, the ND has an average magnetic field of $1.28 \mathrm{~T}$ [50]. This upstream section has 120 planes. These planes are instrumented with a pattern of full $U$-view (FU), full $V$-view (FV), partial $U$-view (PU), and partial $V$ view $(\mathrm{PV})$ scintillator modules. The configuration of these active planes is shown in Fig. 2.15. The calorimeter active planes are arranged in groups of ten of the following pattern: FU-PV-PU-PV-PU-FV-PU-PV-PU-PV. The calorimeter is divided into three sections: planes 1-20 are the veto section, planes 21-60 are the target section, planes 61-120 measure hadronic showers from neutrino interactions in the target section. The spectrometer section, planes 121-281, follows the same 10-plane pattern as the calorimeter but with the partial planes removed. This more coarsely instrumented section is only used for tracking muons. The ND uses Hamamatsu M64 PMTs with each strip mapping to one of the PMT's 64 pixels $[51,54]$. Since the ND is smaller than the FD, the scintillator strips were able to be read out from one end, and the opposite end of the strip was covered with aluminized Mylar to reflect light that traveled to the uninstrumented end. 

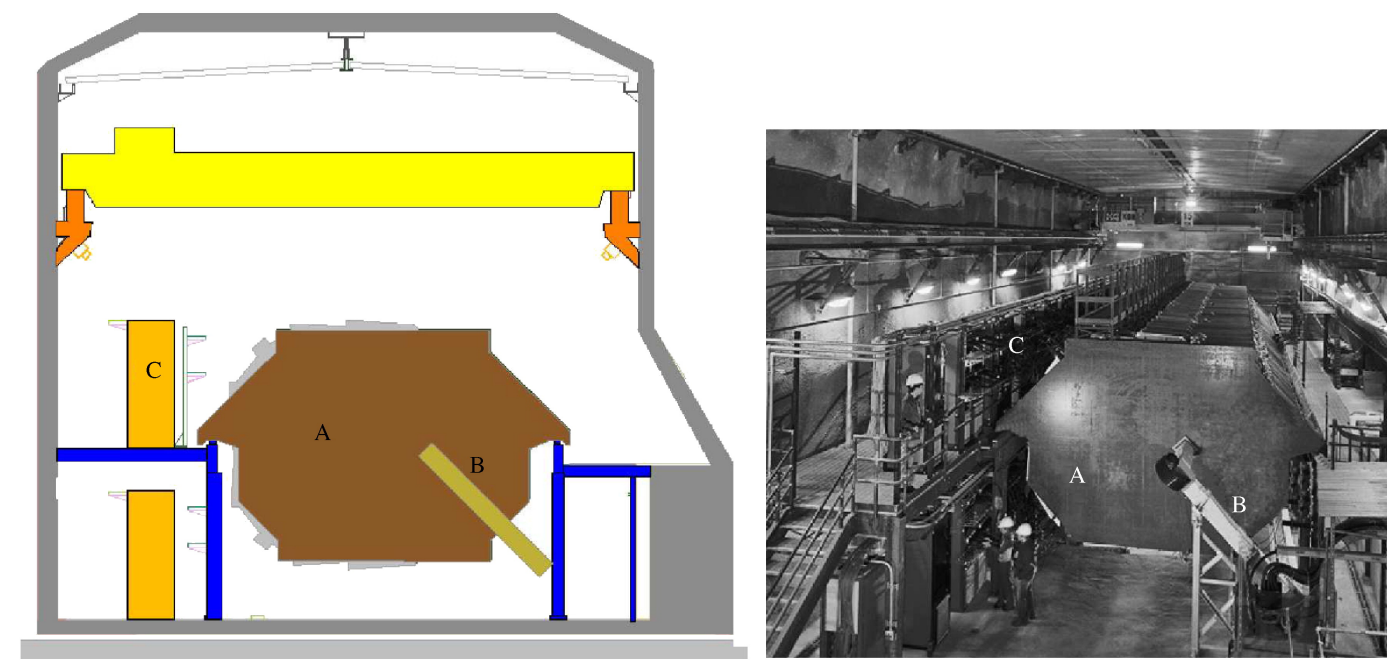

Figure 2.14: The MINOS near detector. On the left is a labeled drawing of the detector pictured on the right. This perspective is looking in the direction of the neutrino beam toward the far detector location. "A" is the furthest upstream steel plane of the detector. " $\mathrm{B}$ " is the magnet coil. " $\mathrm{C}$ " is an electronics rack on the elevated walkway. This figure is from Ref. [50].

\subsubsection{Cosmic Ray Veto Shield}

In addition to measuring neutrinos from the NuMI beam, the FD collected a sample of atmospheric neutrinos. These neutrinos are measured in a large background of cosmic rays entering the top of the detector. Since the FD planes were oriented to detect beam neutrinos, the active scintillator edges only accounted for $17 \%$ of the surface area of the detector's sides. With such little coverage, steep cosmic rays could easily enter the detector through air gaps or steel planes and be mistaken for an atmospheric neutrino interaction.

To reduce the cosmic ray background, the FD was instrumented with a cosmic ray veto shield. The shield, shown in Fig. 2.16, covered the top and 

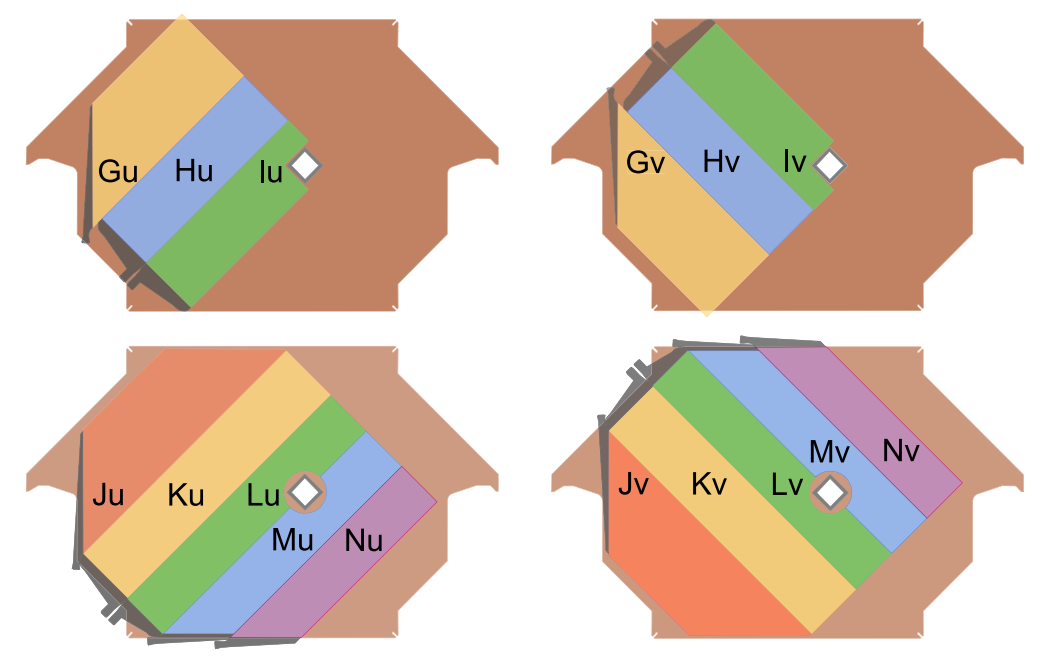

Figure 2.15: The four scintillator plane configurations used in the MINOS near detector. The planes are the top are referred to as partial views, and the planes on the bottom are referred to as full views. The $U$-view planes are on the left, and the $V$-view planes are on the right. The individual panels have a label from $\mathrm{G}$ to $\mathrm{N}$ to denote different shape panels used to construct the plane configuration. Full and partial view configurations used in the ND active planes. The partial view planes are oriented so that they cover the beam center. The full view planes cover the beam center and extend beyond the coil hole of the detector. Drawing from Ref. [50].

sides of the FD including the gap between supermodules. It was assembled from the same $\mathrm{C}$ and $\mathrm{E}$ type scintillator modules in Fig. 2.13 used for the FD scintillator planes. The $8 \mathrm{~m}$ modules were oriented with the scintillator strips parallel to the z-axis of the FD and overlapped at the center of each supermodule to minimize gaps. The sections of the shield directly above the detector were arranged in a double layer to improve tagging efficiency where the cosmic ray flux was the highest, and the vertical sections on either side had a single layer of modules. 
The shield efficiency was measured using a sample of stopping muons. The efficiency is defined as the fraction of muons that satisfied the cosmic ray veto criteria. Figure 2.17 shows the efficiency fell over time starting from $97.1 \%$ in 2003 to $95 \%$ by 2016 . For the previously analyzed exposure from 2003 to 2014, the average shield efficiency was 96.4\%. For the new exposure from February 2014 to June 2016, the average efficiency was 95.2\% [55]. The average efficiency over the entire exposure from 2003 to 2016 was $96.2 \%$ [56].

\subsection{Events and Reconstruction}

The MINOS detectors have the ability to identify three types of neutrino interactions. The design of the detectors is optimized for $v_{\mu}$-CC interactions. A simulation of a $v_{\mu}$ CC interaction is shown in Fig. 2.18. These types of events in the MINOS detectors are characterized by a muon track and a hadronic shower at the track vertex. Muon neutrino CC events serve as the primary signal for this three-flavor neutrino analysis which measures $v_{\mu}$ disappearance. Neutral-current interactions create a hadronic shower in the detectors. An example of a simulated NC event is shown in Fig. 2.19. This class of event is the principal background when trying to identify $v_{\mu}$ CC interactions in the MINOS detectors. Neutral current events are also an important background for $v_{\mathrm{e}} \mathrm{CC}$ events. Figure 2.20 shows a simulated $v_{\mathrm{e}} \mathrm{CC}$ event. Both NC and $v_{\mathrm{e}} \mathrm{CC}$ events are characterized by showers in the detector; however, the electromagnetic component of the $v_{\mathrm{e}} \mathrm{CC}$ events makes the resulting showers more compact. The analysis in this work makes uses of the $v_{\mathrm{e}} \mathrm{CC}$ 


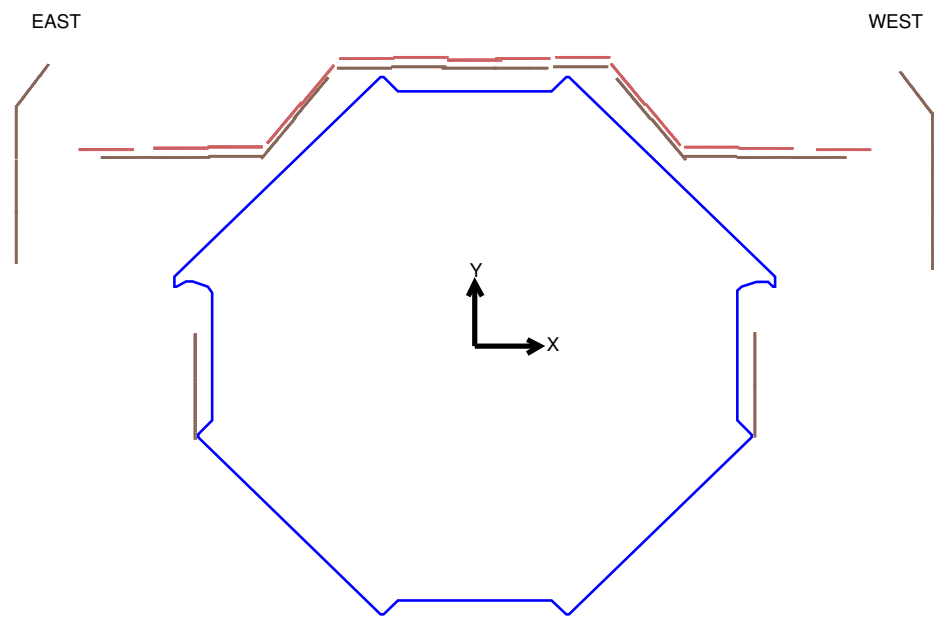

Figure 2.16: Schematic of the Far Detector cosmic ray veto shield looking toward Fermilab. From Ref. [50].

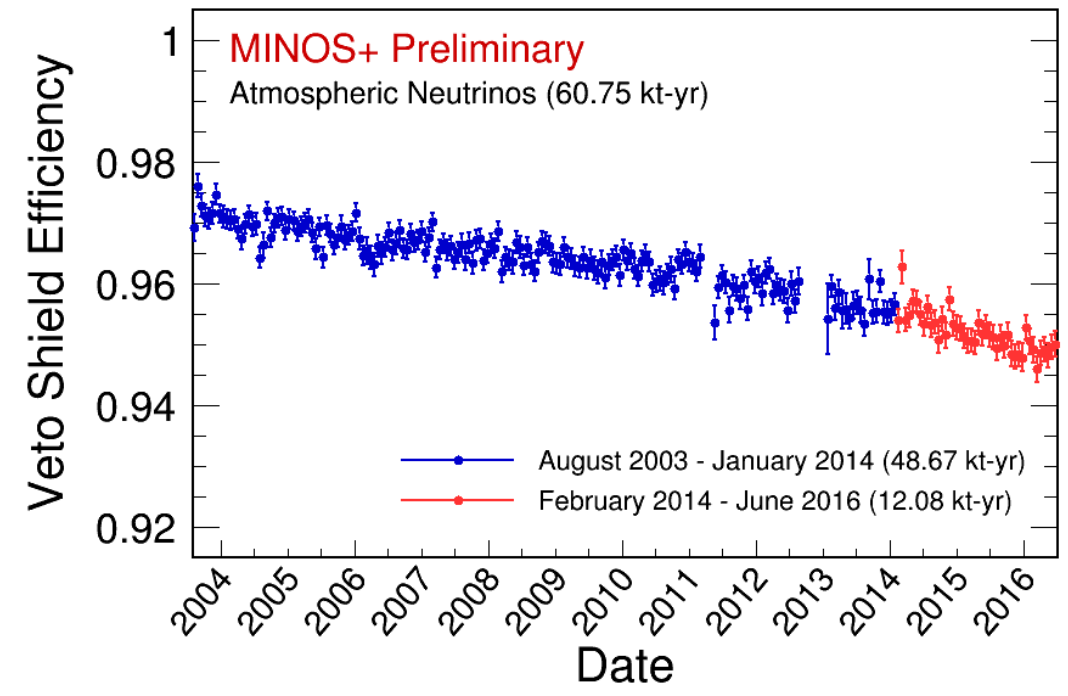

Figure 2.17: Plot of the average veto shield efficiency measured using stopping cosmic-ray muons as a function of time for the complete exposure period. The points shown in blue correspond to the previously analyzed data while the red points represent the new data from February 2014 to June 2016. The average efficiency is calculated to be $96.2 \%$. Figure from Ref. [56]. 


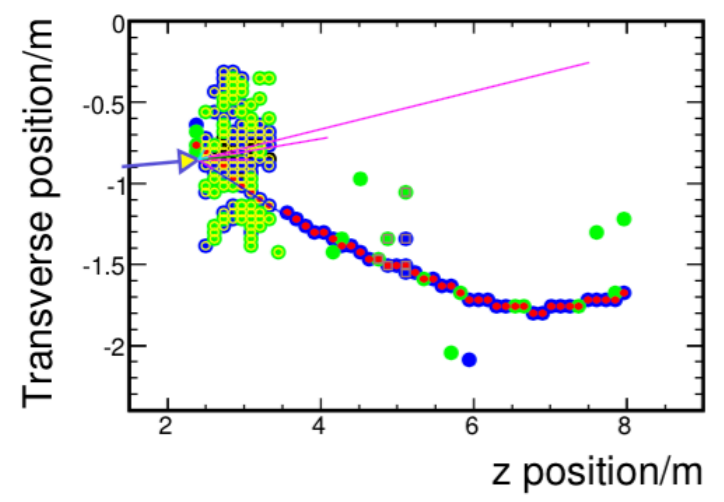

Detected energy depositions

- Deposition $<2.0$ pe

- $2.0<$ Deposition $<20.0$ pe

- Deposition $>20.0$ pe

Reconstruction

- Reconstructed track hit Reconstructed shower hit Monte Carlo Truth $\rightarrow$ Initial $v_{\mu}$ $-\mu^{-} \quad-\pi^{ \pm}$

Figure 2.18: Event display of a simulated $\boldsymbol{v}_{\mu}$ charged-current interaction in a MINOS detector. The display shows the characteristic bending muon track of a $v_{\mu}$ charged current interaction. Event display from [57].

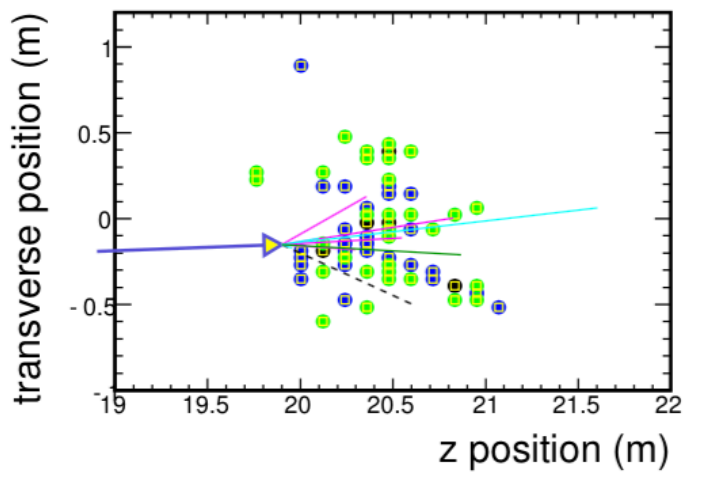

Detected energy depositions

Deposition $<2.0$ pe

- $2.0<$ Deposition $<20.0$ pe

- Deposition $>20.0$ pe

Reconstruction

- Reconstructed track hit Reconstructed shower hit

Monte Carlo Truth

$\rightarrow$ Initial $v_{\mu} \quad \cdots$ Outgoing $v$

$\begin{array}{ll}\pi^{ \pm} & -\mathrm{n} \\ \mathrm{p} & -\pi^{0}\end{array}$

Figure 2.19: Event display of a simulated neutral-current interaction in a MINOS detector. The display shows the hadronic shower characteristic of neutral current interactions. Event display from [57]. 


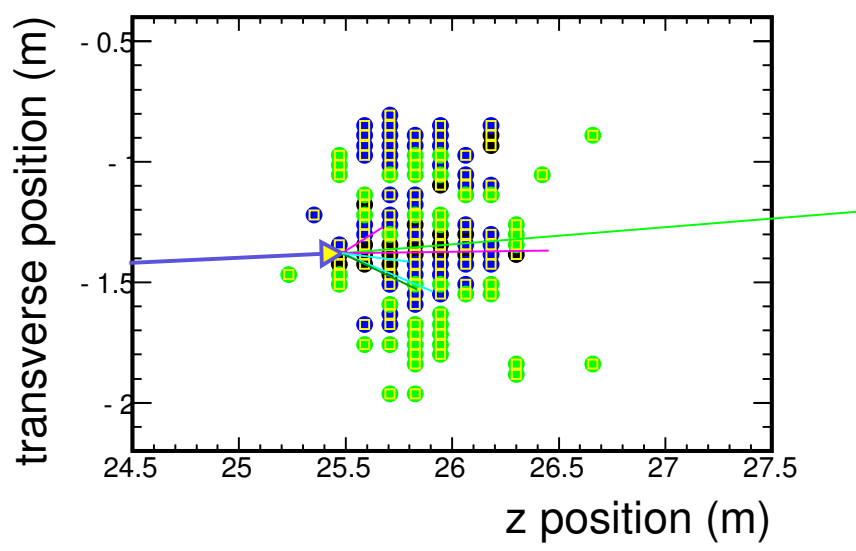

Detected energy depositions

- Deposition $<2.0$ pe

- $2.0<$ Deposition $<20.0$ pe

- Deposition $>20.0$ pe

Reconstruction

- Reconstructed track hit Reconstructed shower hit

Monte Carlo Truth

$\rightarrow$ Initial $v_{\mu}$

$\begin{array}{ll}\mathrm{e}^{ \pm} & \mathrm{p} \\ \pi^{ \pm} & =\pi^{0}\end{array}$

Figure 2.20: Event display of a simulated $v_{\mathrm{e}}$ charged-current interaction in a MINOS detector. Charged current $v_{\mathrm{e}}$ interactions produce a shower in the detector like neutral current interactions; however, these showers have an electromagnetic component in addition to the hadronic activity. Event display from [57].

events measured by MINOS [58].

These events are described in components of tracks and showers. Tracks are reconstructed by using a Kalman filter [59]. The filter accounts for how muons travel through the detector materials and magnetic field. In addition, the filter accounts for uncertainties in the trajectory of a muon due to multiple scattering and ionization effects. For each track that is successfully reconstructed, the filter estimates a state vector at each detector plane that includes the position and the charge to momentum ratio along with their uncertainties. The vertex state vector thus provides the track energy calculation based on its curvature through the magnetic field as well as the charge of the track. However, if the track is fully contained within the detector then the 
track momentum is determined based on the range [60].

Any remaining energy depositions in the detector are grouped together. These hits make up the shower. If a track hit has more energy than predicted for a muon, then the additional energy is added to the shower. The calorimetric energy of the shower is then defined as the sum of the energy from the shower hits and the additional energy in the reconstructed track if it exists. Early MINOS analyses used this calorimetric energy to measure the shower energy. Later MINOS developed a multivariate $k$-Nearest Neighbors $(k \mathrm{NN})$ algorithm [61] to estimate the shower energy. Both MINOS and MINOS+ use the same shower features as selection variables to estimate the shower energy: the deweighted energy deposited within $1 \mathrm{~m}$ of the track vertex, the calorimetric energy of the two largest showers in the event, and the length of the primary shower [62]. Deweighted energy [59] refers to a procedure to account for the nonlinear response of the detector to lower energy events. In this procedure, energy for individual shower hits is raised to a power smoothly ranging from 0 for lower energy hit to 1 for higher energy hits before being summed to find the shower energy.

The $k \mathrm{NN}$ algorithm compares the selection variables from a test event to a standardized population of events called a training set. In this case the test event is a measured shower and the training set is made from simulation. The aim is to find a subset of the training set that is similar to the test event, called the $k$ nearest neighbors. This algorithm uses the Euclidean metric to 
determine the nearest neighbors defined as

$$
d=\sqrt{\sum_{i} \frac{x_{i}-y_{i}^{2}}{\sigma_{i}^{2}}}
$$

where $x_{i}$ is the $i^{\text {th }}$ feature variable of the training point, $y_{i}$ is the $i^{\text {th }}$ feature variable of the test point, and $\sigma_{i}$ is the standard deviation of the $i^{\text {th }}$ variable for the training set. It is important to normalize the sum in Eq. 2.2 by a term such as the standard deviation of the selection variable to avoid bias from incompatible units. Then the $k$ nearest neighbors are those $k$ training points with the smallest values from Eq. 2.2. The estimated shower energy is then the mean true shower energy from the $k$ nearest neighbors.

The selection variables and number of neighbors for MINOS were chosen to optimize sensitivity to the muon neutrino disappearance oscillation parameters. The $k \mathrm{NN}$ algorithm from MINOS had to be re-optimized for MINOS+ [63] to account for the higher energy neutrino beam using a training set from the MINOS+ simulation. For MINOS, $k=400$ was found to optimize the sensitivity while $k=440$ optimized the MINOS+ sensitivity to the oscillation parameters.

This energy estimator is biased toward energies around the peak of the neutrino beam energy since this is where the majority of simulated events are found. The result is that the shower energy for events below the beam peak gets overestimated, and just above the beam peak is underestimated. This bias must be corrected in order to prevent loss of sensitivity to oscillation parameters $[62,63]$. 
Once an event has been reconstructed, the neutrino energy is the sum of the track energy and shower energy. 


\section{Chapter 3}

\section{The Data Set}

The three-flavor oscillation analysis uses data sets from both the NuMI beam and atmospheric neutrino exposures. The medium-energy $v_{\mu}$-mode beam data from MINOS+ is analyzed on its own and with the full available data set from the MINOS beam and the FD atmospheric neutrino exposure. Together MINOS and MINOS+ measure the first oscillation maximum with thousands of neutrino events. The 11 years of atmospheric neutrino data offer additional information over a wide range of $L / E$ values. The beam and atmopsheric neutrino samples are analyzed separately and then fit together to provide increased sensitivity to the three-flavor neutrino oscillation parameters. All data sets used in this analysis are described in the following sections.

\subsection{Beam Exposure}

The beam exposure is measured using the number of protons on target (POT). The MINOS and MINOS+ target exposures are shown in Fig. 3.1 separated by neutrino beam configuration. From 2005 to 2016, the MINOS and MINOS+ targets were exposed to more than $26 \times 10^{20}$ POT. This analysis

adds $9.69 \times 10^{20}$ POT of medium-energy neutrino beam data from MINOS+ 
to the MINOS beam data previously published [64]. The accumulated POT

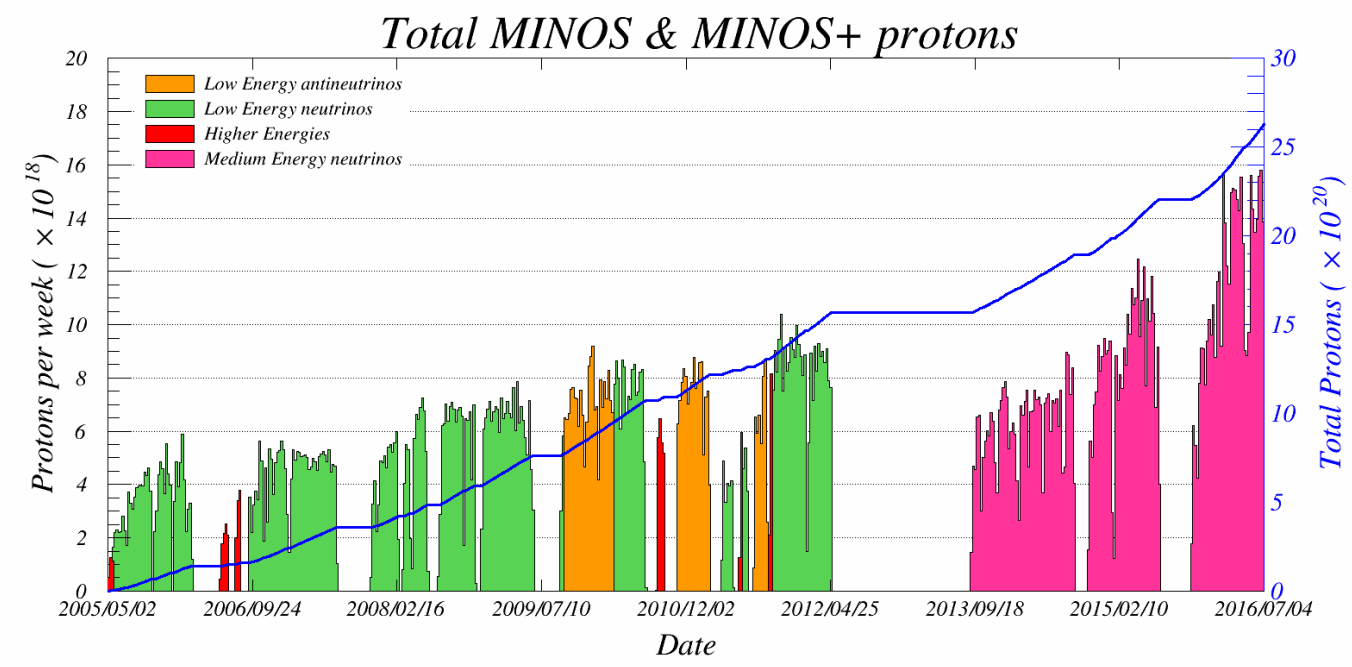

Figure 3.1: The total number of protons on the NuMI target over both the MINOS and MINOS+ runs. The plot covers the entire running time from 2005 to 2016. MINOS took beam data in different configurations: low-energy $\boldsymbol{v}_{\mu^{-}}$ mode (green), low-energy $\bar{v}_{\mu}$-mode (orange), and special configurations (red) which include high-energy $\bar{v}_{\mu}$-mode or the focusing horns turned off. The medium-energy $v_{\mu}$-mode POT for MINOS+ is shown in pink. The individual configurations are plotted with the POT per week, and the cumulative amount of POT is plotted in blue.

for MINOS+ is shown in Fig. 3.2. The beam data are organized in run periods, or runs, which are labeled numerically from 1 to 13. Both Arabic and Roman numerals are used when labeling run periods. MINOS+ is made up of runs 11 to 13 . Figure 3.3 compares the selected $v_{\mu}$ spectrum from the $\boldsymbol{v}_{\mu}$-mode beam at the ND of MINOS to MINOS+ by run. The FD beam exposures used in this analysis are shown in Table 3.1 by run period along each run configuration. 


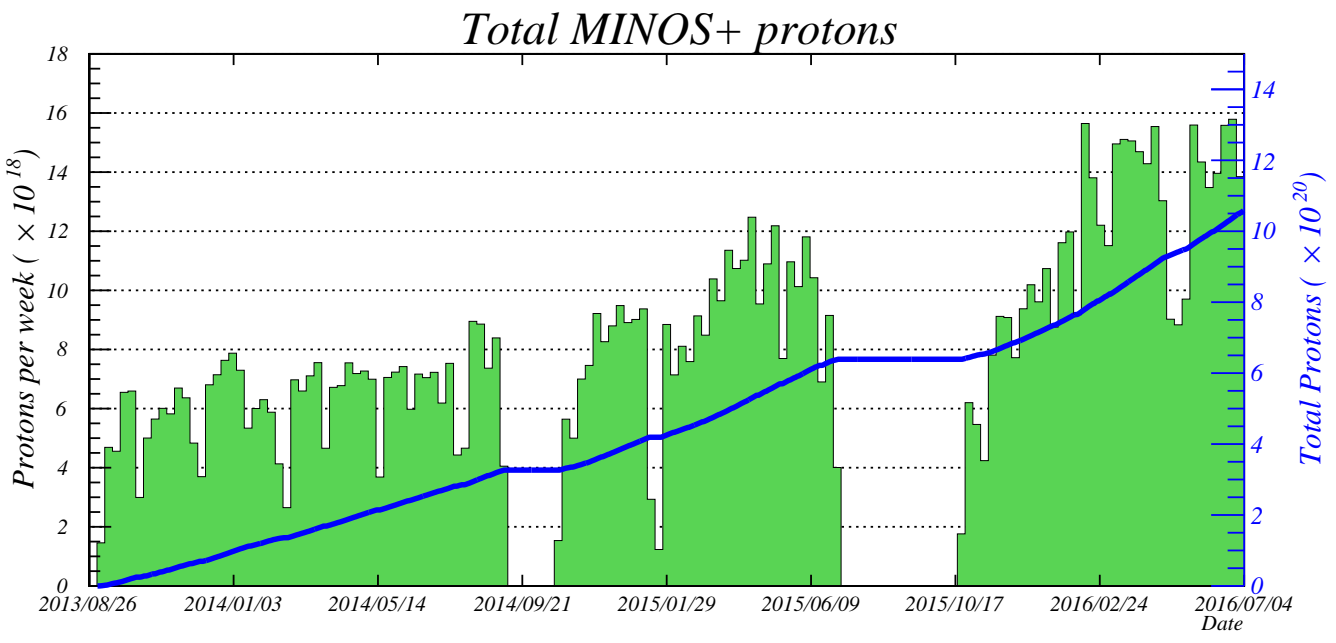

Figure 3.2: The total number of protons on the NuMI target during MINOS+. The plot covers the entire MINOS+ running from 2013 to 2016. The POT per week is plotted in green, and the cumulative amount of POT is plotted in blue.

\subsection{Atmospheric Exposure}

The atmospheric neutrino exposure of the FD is measured in units of kiloton-year (kt.yr) and excludes windows of time coincident with the neutrino beam. Figure 3.4 shows the atmospheric neutrino event rate at the FD for the full exposure from 2003 to 2016 . From the full $60.75 \mathrm{kt} \cdot \mathrm{yr}$ data set, an average of 0.80 events per live-day satisfy the atmospheric neutrino selection criteria. This analysis adds $12.08 \mathrm{kt} \cdot \mathrm{yr}$ to the previously analyzed atmospheric neutrino data and $22.87 \mathrm{kt} \cdot \mathrm{yr}$ to the published MINOS three-flavor oscillation analysis [64]. The FD atmospheric neutrino exposures are summarized in Table 3.2. 


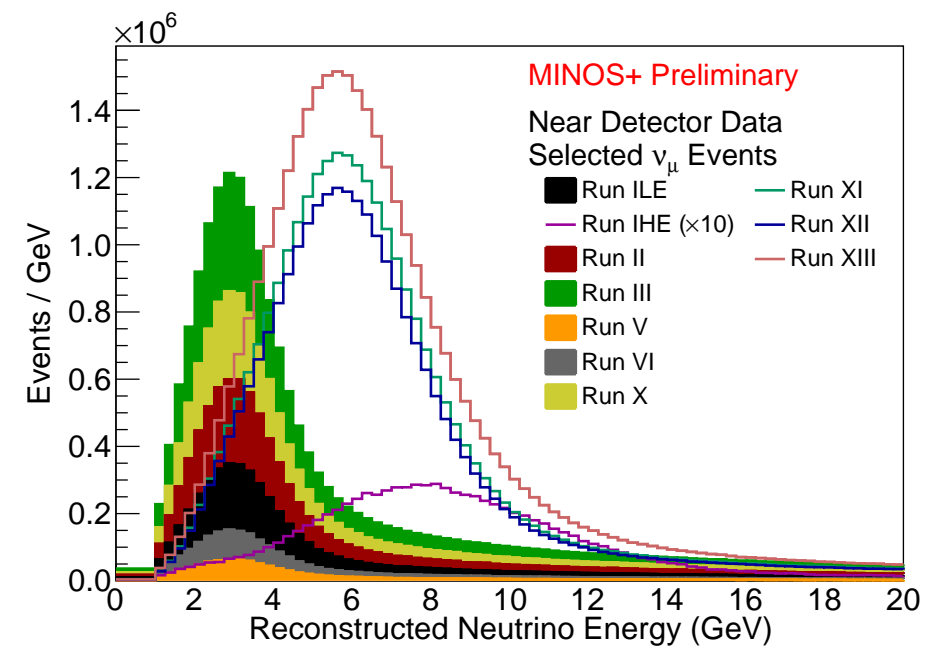

Figure 3.3: Combined MINOS $v_{\mu}$ CC energy spectrum from the $v_{\mu}$-mode beam at the ND compared to the $v_{\mu}$ CC spectra from the MINOS+ runs. The individual MINOS low-energy run spectra are stacked solid histograms. The MINOS high-energy spectrum is multiplied by a factor of 10 and shown in purple. The MINOS+ run spectra are plotted individually as the line histograms in green, blue, and red.

\subsection{Summary}

Table 3.3 summarizes the distinctive samples used in this three-flavor analysis. Muon neutrino disappearance is measured using $v_{\mu}$ and $\bar{v}_{\mu}$ events detected from $9.69 \times 10^{20} \mathrm{POT}$ of the MINOS+ medium-energy $v_{\mu}$-mode beam. The measurement of muon neutrino disappearance is improved by analyzing the MINOS+ data together with the MINOS beam data and the full FD atmospheric exposure of $60.75 \mathrm{kt} \cdot \mathrm{yr}$. The MINOS beam exposure used in the joint analysis is $10.71 \times 10^{20} \mathrm{POT}$ of low-energy and high-energy $\nu_{\mu}$-mode beam and $3.36 \times 10^{20} \mathrm{POT}$ of low-energy $\bar{v}_{\mu}$-mode beam. 
Table 3.1: MINOS and MINOS+ beam exposure at the FD by run period along with each run configuration $[65,66]$. The neutrino beam energy is classified as LE for low-energy, ME for medium-energy, and HE for high-energy.

\begin{tabular}{lllc}
\hline Run & Mode & Energy & Protons on Target $\left(\times 10^{18}\right)$ \\
\hline I & $v_{\mu}$ & LE & 126.93 \\
I & $v_{\mu}$ & HE & 15.31 \\
II & $v_{\mu}$ & LE & 194.27 \\
III & $v_{\mu}$ & LE & 388.71 \\
IV & $\bar{v}_{\mu}$ & LE & 170.85 \\
V & $v_{\mu}$ & LE & 45.89 \\
VI & $v_{\mu}$ & LE & 61.62 \\
VII & $\bar{v}_{\mu}$ & LE & 124.08 \\
IX & $\bar{v}_{\mu}$ & LE & 40.80 \\
X & $v_{\mu}$ & LE & 238.31 \\
XI & $v_{\mu}$ & ME & 298.52 \\
XII & $v_{\mu}$ & ME & 281.72 \\
XIII & $v_{\mu}$ & ME & 389.12 \\
\hline
\end{tabular}

Table 3.2: MINOS and MINOS+ atmospheric neutrino exposures at the FD $[56,67,68]$.

\begin{tabular}{llll}
\hline Data Set & Start & End & $\mathrm{kt} \cdot \mathrm{yr}$ \\
\hline 2012 & $2003-08-01$ & $2011-03-07$ & 37.88 \\
2014 & $2011-03-08$ & $2014-01-31$ & 10.79 \\
New & $2014-02-01$ & $2016-06-30$ & 12.08 \\
\hline Total & $2003-08-01$ & $2016-06-30$ & 60.75 \\
\hline
\end{tabular}




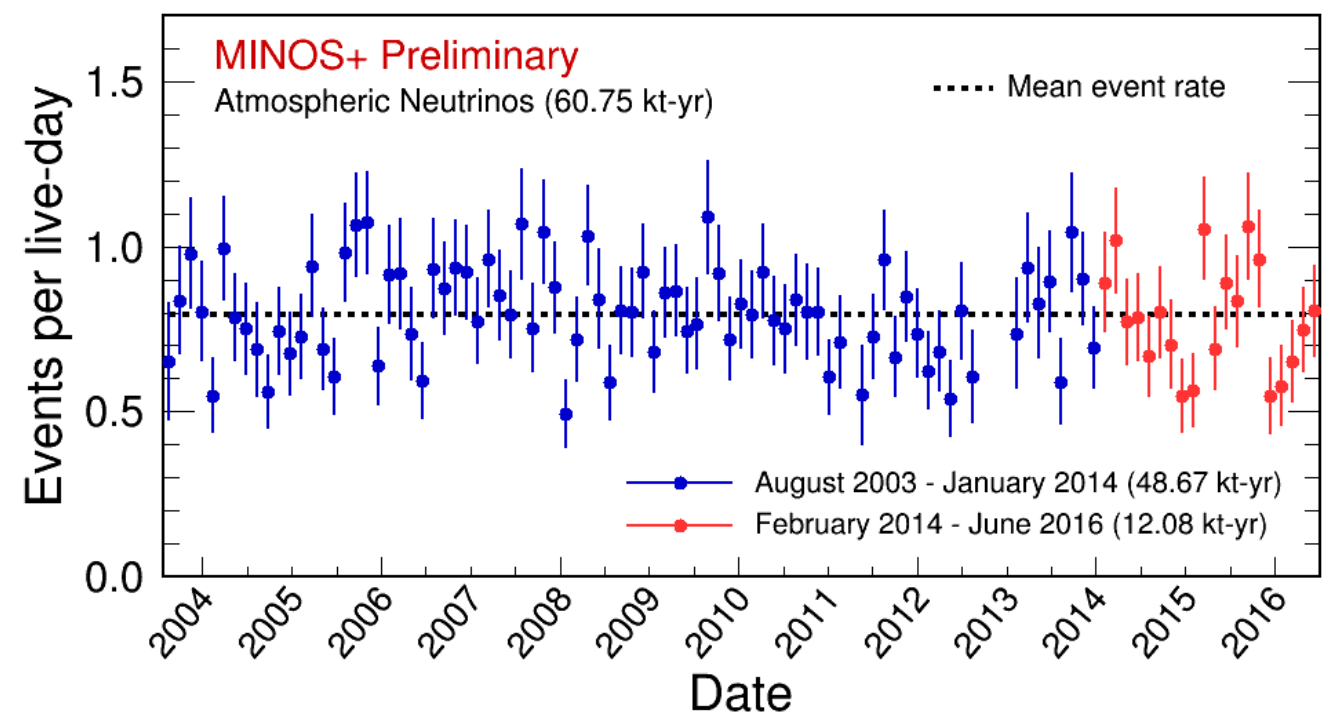

Figure 3.4: The number of selected atmospheric neutrino events at the FD per live-day of running, for the full duration of data taking. The points shown in blue correspond to the previously analyzed data while the red points represent the new data (February 2014 to June 2016). Events passing the selection cuts occur at a mean rate of 0.80 per live-day. This figure is from Ref. [56].

Table 3.3: Distinct MINOS and MINOS+ exposures used in the three-flavor analysis. The neutrino beam energy is classified as LE for low-energy, ME for medium-energy, and HE for high-energy.

\begin{tabular}{lr}
\hline Sample & Exposure \\
\hline LE + HE $v_{\mu}$-mode & $1071.04 \times 10^{18} \mathrm{POT}$ \\
LE $\bar{v}_{\mu}$-mode & $335.73 \times 10^{18} \mathrm{POT}$ \\
ME $\boldsymbol{v}_{\mu}$-mode & $969.36 \times 10^{18} \mathrm{POT}$ \\
Atmospheric neutrinos & $60.75 \mathrm{kt} \cdot \mathrm{yr}$ \\
\hline
\end{tabular}




\section{Chapter 4}

\section{MINOS+ Event Selection}

For both the NuMI amd atmospheric neutrino sources, there are two general categories of events used in this analysis that describe where the neutrino interaction occurred: Contained Vertex $(\mathrm{CV})$ and non-fiducial. Events that are classified as CV have an interaction vertex in the fiducial volume of the detector with no apparent initial particle. On the other hand, non-fiducial events are tracks that enter the detector from outside the fiducial volume. In this analysis, non-fiducial events always refer to muon-like events.

The CV sample for this analysis includes $v_{\mu} \mathrm{CC}$ and $\bar{v}_{\mu} \mathrm{CC}$ interactions from both the beam and atmosphere. The atmospheric neutrino data also has a sample of CV shower events which contains $v_{\mathrm{e}} \mathrm{CC}, \bar{v}_{\mathrm{e}} \mathrm{CC}$, and $\mathrm{NC}$ interactions. The analysis also uses constraints obtained from the analysis of CV $v_{\mathrm{e}} \mathrm{CC}$ and $\bar{v}_{\mathrm{e}} \mathrm{CC}$ events from MINOS [58].

Non-fiducial events are an important event class from the atmospheric neutrino sample since atmospheric neutrinos coming from the other side of Earth can interact in the rock surrounding the FD creating high-energy muons that enter the detector. These muons are called neutrino-induced muons (NIMs), and they enter the detector from below and horizontally. Likewise, 
the neutrino beam can create NIMs that enter the detector. In addition to NIMs, the beam non-fiducial event sample includes events where the vertex is outside the fiducial volume, and the event may only be partially contained. Only MINOS $v_{\mu}$-mode beam non-fiducial events were analyzed [69].

This work is chiefly concerned with the selection of $\mathrm{CV} v_{\mu} \mathrm{CC}$ and $\bar{v}_{\mu}$ CC events from the medium-energy neutrino beam measured by MINOS+, which will be explained in the following sections. The selection for other events utilized will be summarized to clearly define the events used in the analysis. All the event selection procedures have a similar form. First, there is a preselection which checks the quality of the data and the state of the detector. There are also initial criteria at this stage to eliminate events that are poor candidates. After the preselector filters out bad events, the candidate events are subject to a selection tailored to find a particular class of event.

\subsection{MINOS $+v_{\mu}$ CC Selection}

The muon neutrino disappearance analysis uses CC muon neutrino interactions which result in a muon in the final state. An example of such an event is shown in Fig. 2.18. Signal events are characterized by a muon track traversing the detector from the interaction point. The major source of background is contamination from NC events (Fig. 2.19) characterized by only a hadron shower in the detector which can contain short tracks from charged pions or false reconstructed tracks in the shower.

The goal of the selection is to select track-like events. MINOS used 
a multivariate $k \mathrm{NN}$ algorithm [70] to select $v_{\mu} \mathrm{CC}$ and $\bar{v}_{\mu} \mathrm{CC}$ events based on event topology and muon track energy deposition. The event selection algorithm uses the same principles as the $k \mathrm{NN}$ shower energy reconstruction described in Section 2.3. A muon track should cross multiple detector planes, deposit about the same amount of energy in each plane, and only deposit energy along the muon's path. The selection algorithm uses four selection variables which reflect the expected muon track properties: the number of detector planes hit by the track, the average energy deposited per scintillator plane by the track, the track's transverse energy deposition profile, and the variation of the energy deposited along the track. These variables are described in detail in Ref. [70].

Distributions of the MINOS+ selection variables are plotted for the ND in Fig. 4.1 and for the FD in Fig. 4.2. The average energy deposited per scintillator plane by the track is the mean pulse height of the track hits excluding the first $30 \%$ of the track scintillator planes. The track's transverse energy profile is quantified by the ratio of the sum of the track hit pulse heights to the sum of all hits in a four strip window around the track excluding the initial $50 \%$ of the track planes. The variation of the energy deposited along the track, also called the signal fluctuation, is calculated as the ratio of the lower $50 \%$ of track hit pulse heights to the higher $50 \%$ of track hit pulse heights in a four strip wide window around the track excluding the first $30 \%$ of track planes.

The selector training set contains an equal number of signal $v_{\mu} \mathrm{CC}$ 

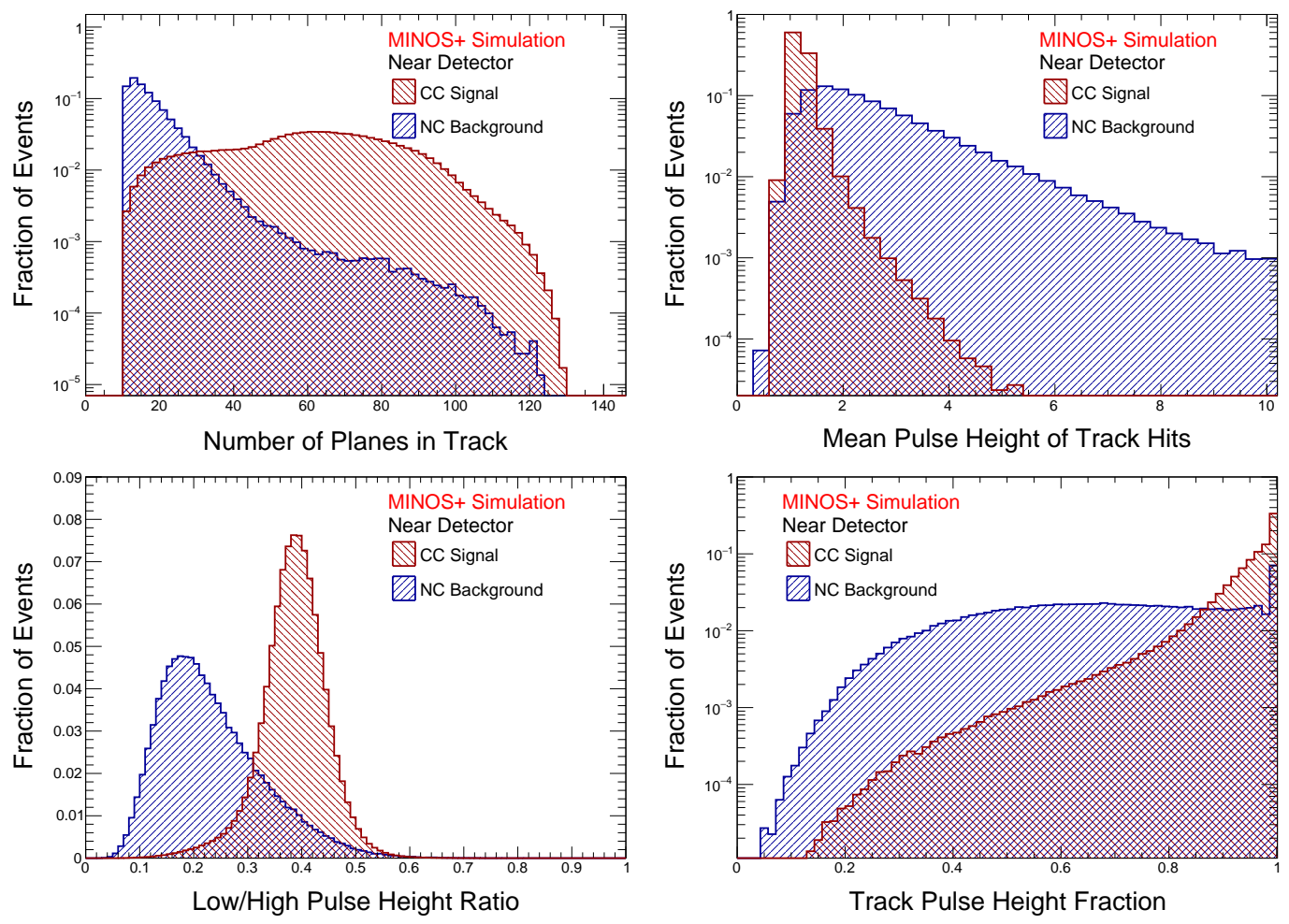

Figure 4.1: MINOS $+k N N$ selection variable distributions from the ND training sample for $v_{\mu} \mathrm{CC}$ signal events and background events. 

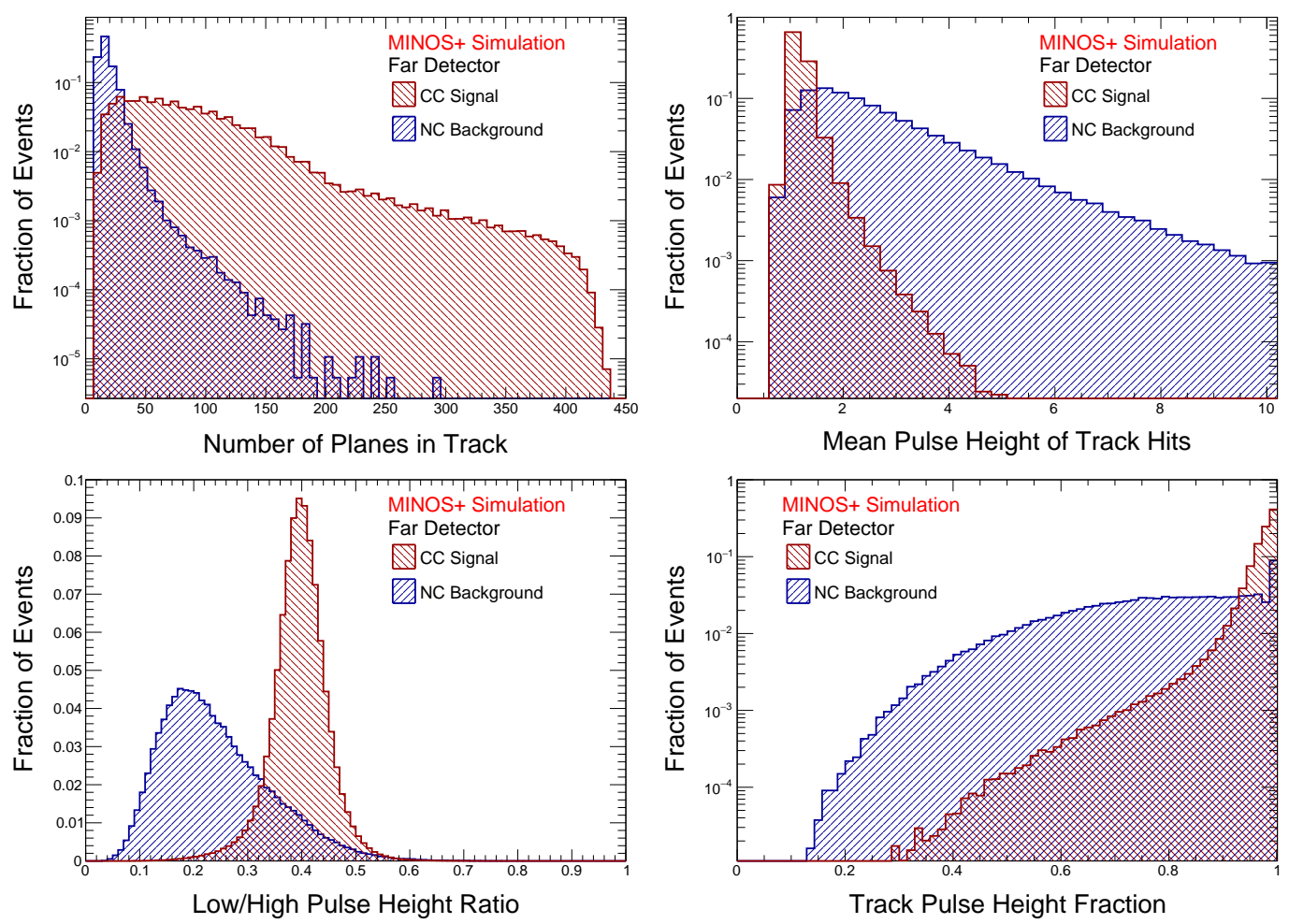

Figure 4.2: MINOS+ $k$ NN selection variable distributions from the FD training sample for $v_{\mu} \mathrm{CC}$ signal events and background events. 
interactions with muon tracks and background interactions without a muon track. The Euclidean metric defined in Eq. 2.2 is used to find the $k$ nearest neighbors to the test event. The MINOS+ selector uses $k=80$ which was found to increase the separation of signal and background for MINOS [70]. The $k$ nearest neighbors are used to define a discriminant variable, $\mathcal{R}$, which is the fraction of signal events in the $k$ nearest neighbors.

$$
\mathcal{R}=\frac{k_{\mathrm{S}}}{k_{\mathrm{S}}+k_{\mathrm{B}}}=\frac{k_{\mathrm{S}}}{k},
$$

where $k_{\mathrm{S}}$ and $k_{\mathrm{B}}$ are the number of signal and background events among the $k$ nearest neighbors to the test event.

The algorithm for MINOS+ was updated to use representative MI$\mathrm{NOS}+\mathrm{CC}$ and $\mathrm{NC}$ events in the training sample, and the selection was optimized to improve the sensitivity to the standard oscillation model [63]. The optimization maximized the $\chi^{2}$ value at a test point well outside the $90 \%$ C.L. from the MINOS measurement using beam and atmospheric neutrinos [69] as a function of the selection parameter $\mathcal{R}$. The value $\mathcal{R}=0.3$ produced the optimum sensitivity [63].

\subsubsection{Fiducial Volumes}

The detector fiducial volumes are defined to select a sample of events whose deposited energy is well contained and can be reconstructed. The dimensions are motivated by the need to contain all energy deposited in the form

of showers within the detector and to ensure there is enough information to 
reconstruct the events track energy. The vertices of selected CV $v_{\mu} \mathrm{CC}$ events from the beam must be located within the detector fidicual volume, which is the same for MINOS and MINOS+.

The ND fiducial volume is a cylinder with $r=0.8 \mathrm{~m}$ centered on the beam spot at $X_{0}=1.4828 \mathrm{~m}$ and $Y_{0}=0.2384 \mathrm{~m}$. The cylinder extends $3.27 \mathrm{~m}$ from steel plane 13 to 68 . This volume is offset from the magnetic coil located at the $X=0$ and $Y=0$. The vertex distributions in $X, Y$, and $Z$ are plotted for $v_{\mu}$ events in Fig. 4.3 and $\bar{v}_{\mu}$ events in Fig. 4.4.

The FD has a fiducial volume for each supermodule. Both volumes are hollow cylinders to exclude the volume near the magnet coil with $r=0.4 \mathrm{~m}$ and $R=3.74 \mathrm{~m}$. The inner radius requirement prevents losing event information in the magnetic coil. The upstream volume is $14.04 \mathrm{~m}$ long extending from steel plane 3 to 239 . The downstream volume is $12.60 \mathrm{~m}$ long extending from steel plane 252 to 464 . The transition between supermodules is visible in the distribution of the track vertex plane of Fig. 4.5 for selected $\gamma_{\mu}$ events as the dip between planes 200 and 250. Figures 4.5 and 4.6 show the vertex distributions plotted for selected $v_{\mu}$ and $\bar{v}_{\mu}$ events from data and MC that has been be weighted by three-flavor oscillation probabilities.

\subsubsection{Preselection}

The MINOS+ preselection requirements are designed to prepare a reliable sample of candidate events with tracks that are coincident with the proton beam and in the beam direction. These criteria address backgrounds from cos- 

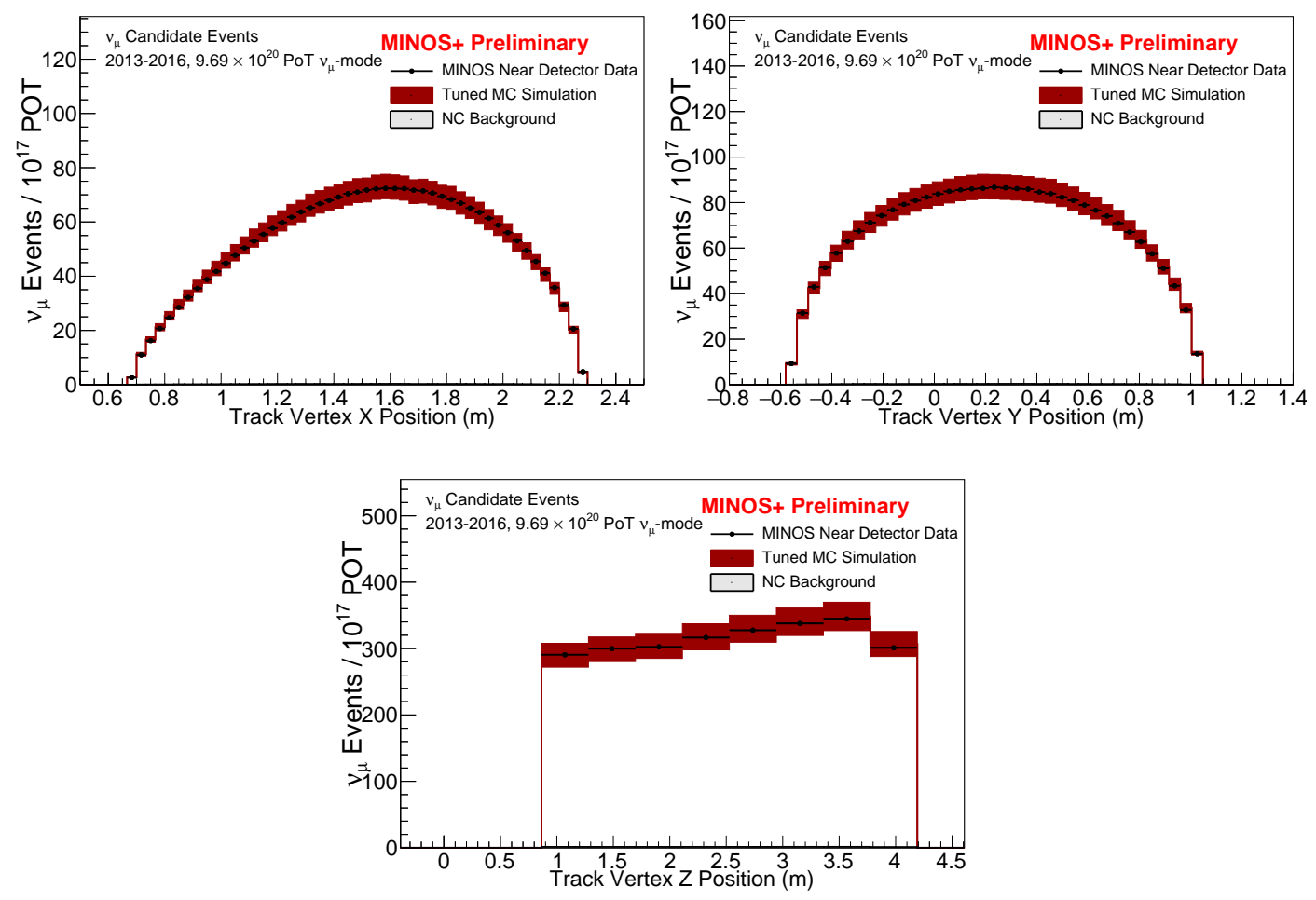

Figure 4.3: Distribution of the $v_{\mu}$-CC event vertex coordinates in the ND for the MINOS+ beam exposure. The $X$ distribution (top left), $Y$ distribution (top right), and $Z$ distribution (bottom) of data and $\mathrm{MC}$ events. $Z=0$ corresponds to the first plane in the detector. 

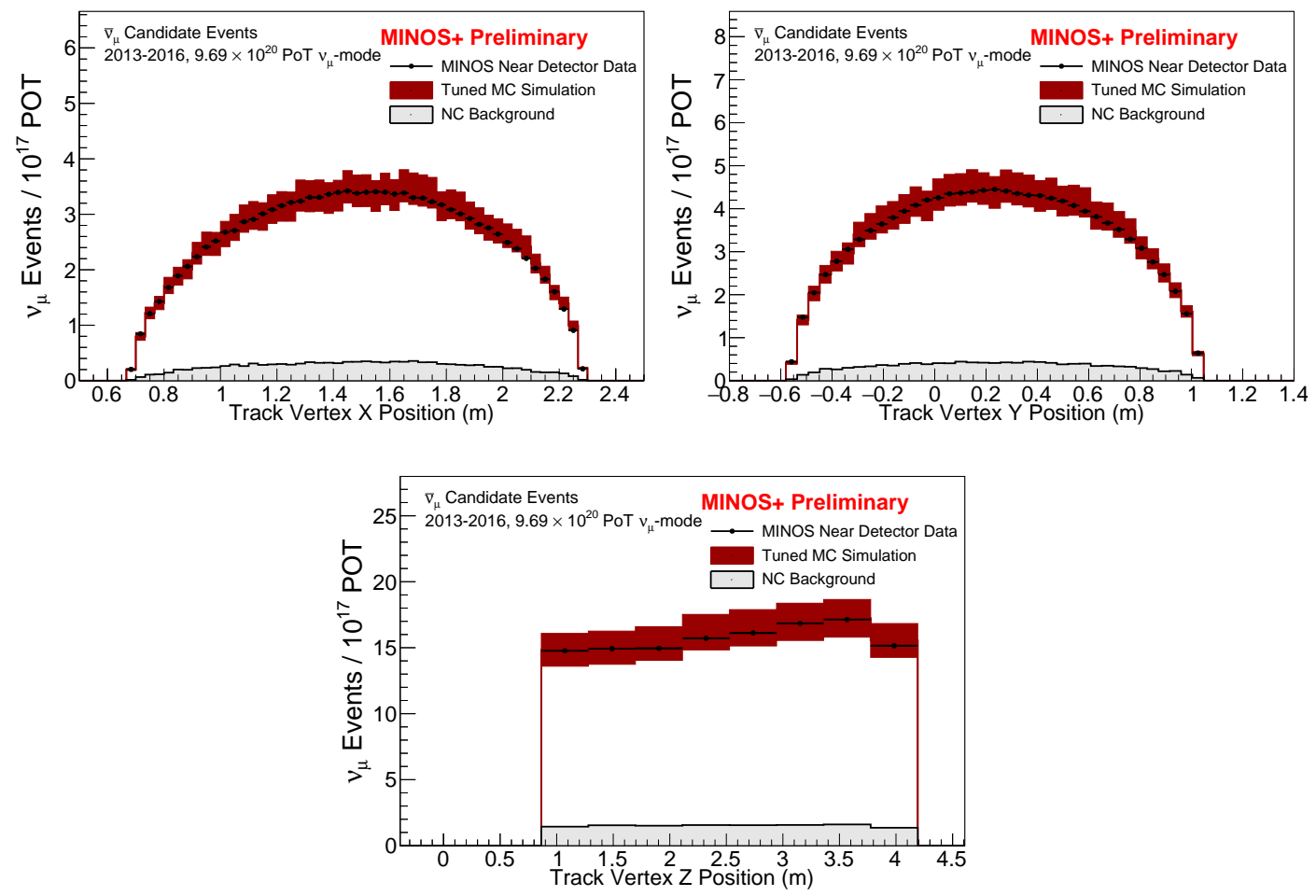

Figure 4.4: Distribution of the $\bar{v}_{\mu}$ CC event vertex coordinates in the ND for the MINOS+ beam exposure. The $X$ distribution (top left), $Y$ distribution (top right), and $Z$ distribution (bottom) of data and $\mathrm{MC}$ events. $Z=0$ corresponds to the first plane in the detector. 

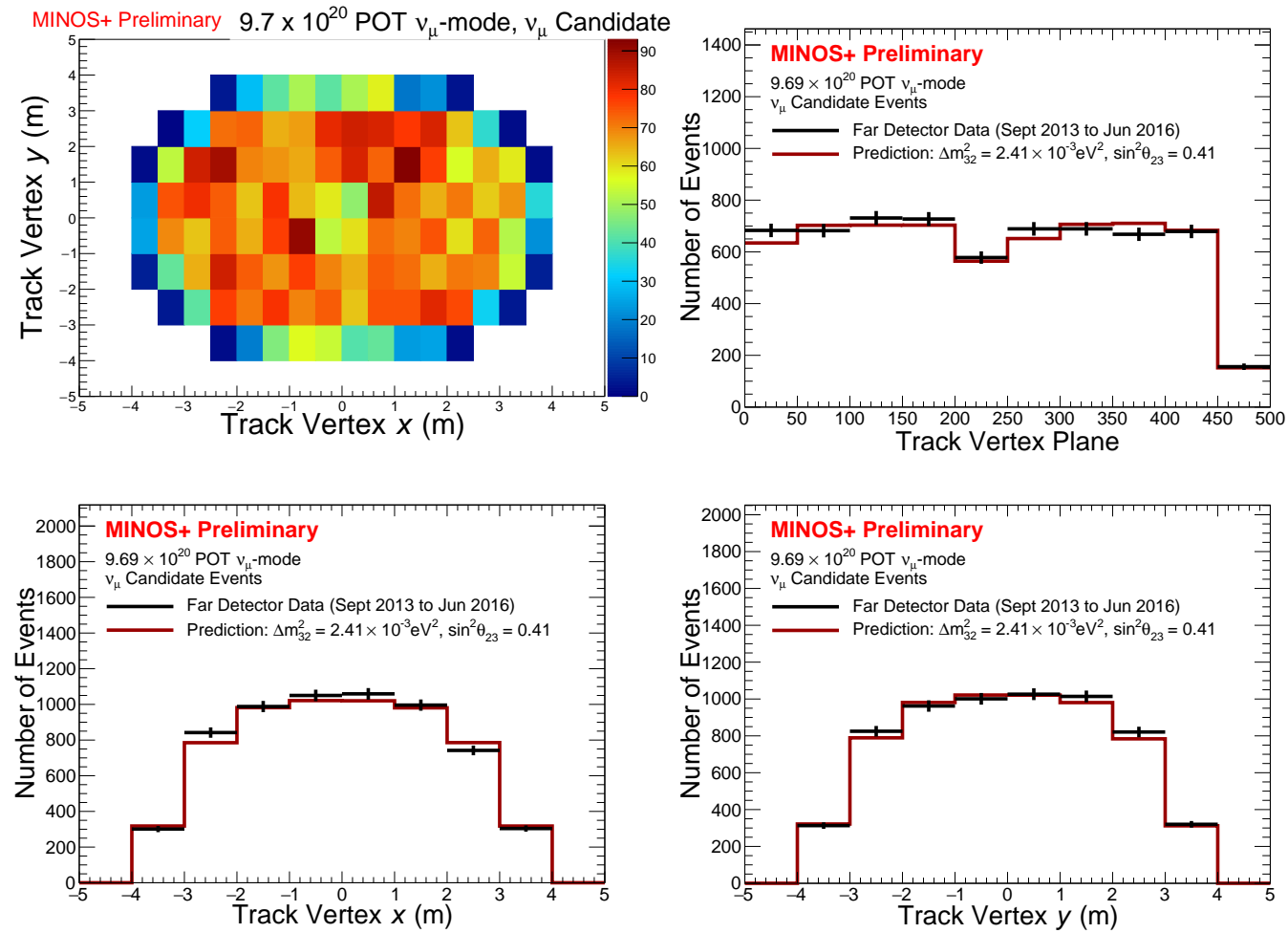

Figure 4.5: Distribution of the $\boldsymbol{v}_{\mu}$-CC event vertex coordinates in the FD for the MINOS+ beam exposure. The $X Y$-view of the vertices for data events is plotted on the top left. The $Z$-plane distribution (top right), $X$ distribution (bottom left), and $Y$ distribution (bottom right) of data and oscillated $\mathrm{MC}$ events. 

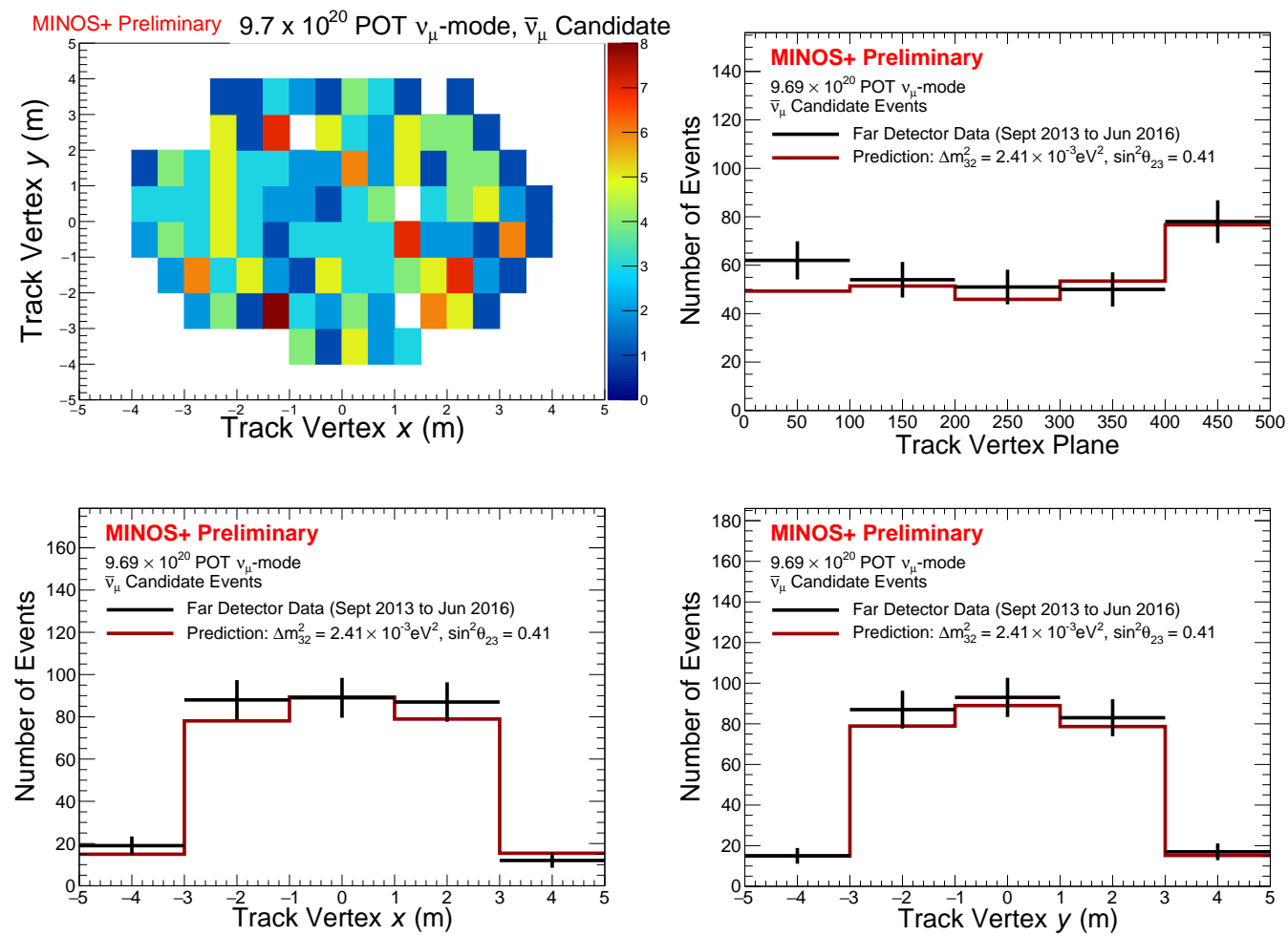

Figure 4.6: Distribution of the $\bar{v}_{\mu}$-CC event vertex coordinates in the FD for the MINOS+ beam exposure. The $X Y$-view of the vertices for data events is plotted on the top left. The $Z$-plane distribution (top right), $X$ distribution (bottom left), and $Y$ distribution (bottom right) of data and oscillated $\mathrm{MC}$ events. 
mic rays and atmospheric neutrinos. MINOS $+v_{\mu} \mathrm{CC}$ candidates must first satisfy the following requirements:

- the neutrino beam must have been functioning within set limits

- the detector must have been functioning properly and ready to measure interactions

- the event vertex must be located in the detector fiducial volume

- the event must have at least one track that is successfully reconstructed

- an FD event must be recorded within $-2 \mu$ s to $+12 \mu$ s of the proton beam spill

- for an FD event, the cosine of the angle between the track and the beam must be $>0.6$

The track requirement is driven by the fact that a muon track is the distinguishing feature of the $v_{\mu}$-CC interactions. At the FD, the timing and track angle requirements ensure that the event came from the neutrino beam as opposed to a cosmic ray or atmospheric neutrino. Figures 4.7 and 4.8 show the distributions of the cosine of the track angle for $v_{\mu}$ and $\bar{v}_{\mu}$ events selected from data and oscillated MC.

\subsubsection{Selection}

The final selection criteria are used to isolate a clean sample of CC events with muon tracks, minimizing contamination from NC interactions. 


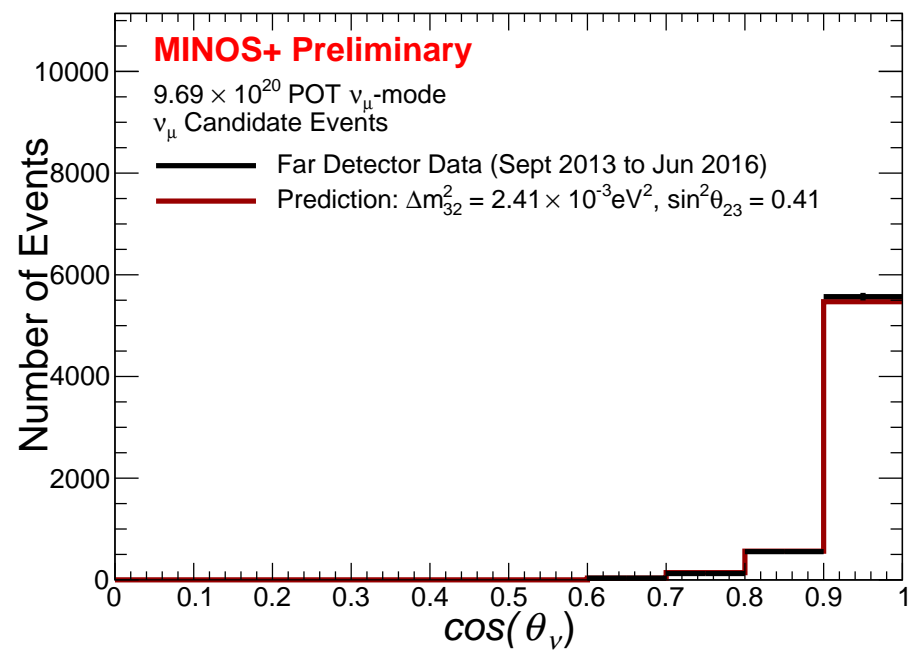

Figure 4.7: Distribution of the cosine of the angle between the $\mu^{-}$track and the beam direction for selected $v_{\mu}$ events in data and oscilalted MC.

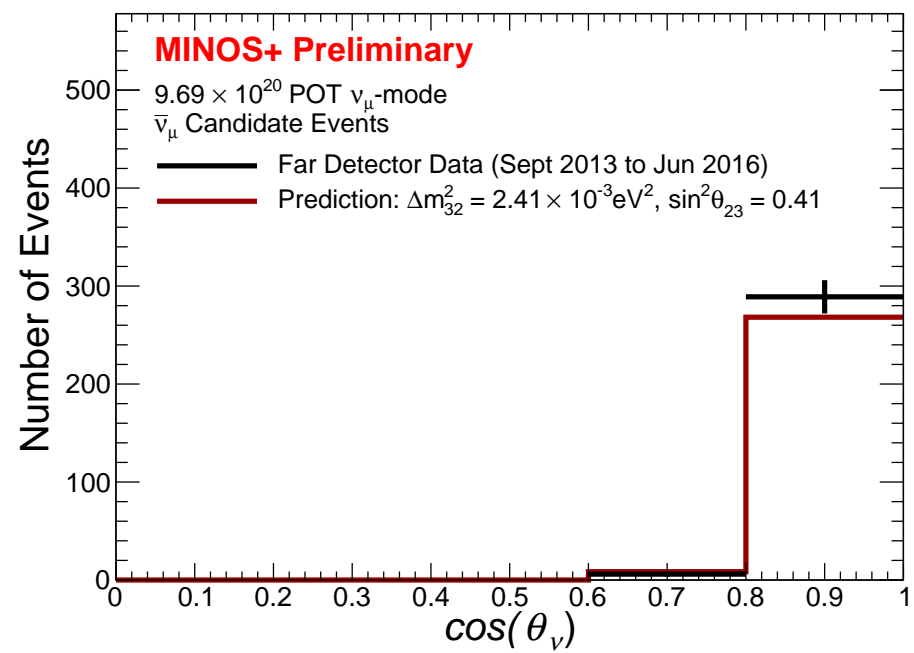

Figure 4.8: Distribution of the cosine of the angle between the $\mu^{+}$track and the beam direction for selected $\bar{v}_{\mu}$ events in data and oscillated MC. 
The track event candidates that satisfy the preselection requirements are then tested with the selection criteria:

- an FD event may be coincident with one other FD event

- if an FD event is coincident with another event, the event's summed pulse height must account for $>75 \%$ of the total pulse height recorded in the detector

- an ND event that exits the side of the calorimeter is rejected

- an ND event with a track that ends in the calorimeter and within $0.6 \mathrm{~m}$ of the coil is rejected

- the event must have $\mathcal{R}>0.3$

The FD requirement on coincident events rejects improperly reconstructed events which are actually part of larger events [71]. At the ND, the requirements make sure that the event energy is contained and can be reconstructed. The requirement on the distance from the coil is related to inaccuracies in the modeling of muon tracks traveling into the coil hole. Finally, $\mathcal{R}$ is used to separate CC-like from NC-like events shown in Figs. 4.9 and 4.10.

The MINOS $+v_{\mu}$ CC selection is different from the MINOS selection in two ways. First, MINOS used two $k$ NN selection algorithms. The MINOS+ $k$ NN selection algorithm uses the same technique as developed in Ref. [70]. The second MINOS $k$ NN algorithm was inspired by the same algorithm, but 
it uses different selection variables to improve the selection of lower energy $v_{\mu}$ CC interactions [72]. This lower energy selector is not necessary for MINOS+ since there are very few low energy events. The second change to the event selection from MINOS to MINOS+ is that the requirement on tracks in the ND ending close to the magnetic coil was loosened for MINOS+ since it improved the agreement between data and simulation [73]. For MINOS, all CC tracks that end within $0.6 \mathrm{~m}$ of the ND magnetic coil are rejected.

\subsubsection{MINOS+ Selection Results}

The selection is evaluated based on its purity and efficiency. Purity is the fraction of true signal events selected,

$$
\frac{\text { number of true signal events selected }}{\text { total number of selected events }}
$$

and efficiency is the fraction of selected true signal events from all the true signal events,

$$
\frac{\text { number of true signal events selected }}{\text { total number of true signal events }} \text {. }
$$

The MINOS+ CC selection has an efficiency of $58.7 \%$ at the ND with a purity of $99.1 \%$. At the FD, the efficiency is $84.2 \%$ and purity is $99.3 \%$. Figure 4.11 shows the efficiency and purity as functions of neutrino energy for each detector. The efficiency at the ND suffers from rejecting signal events that are too close to the magnetic coil. However, the effect is negligible since the ND detects such a large sample of neutrino interactions. Figure 4.12 shows the spectrum of selected $v_{\mu}$ and $\bar{v}_{\mu}$ events from the ND. 


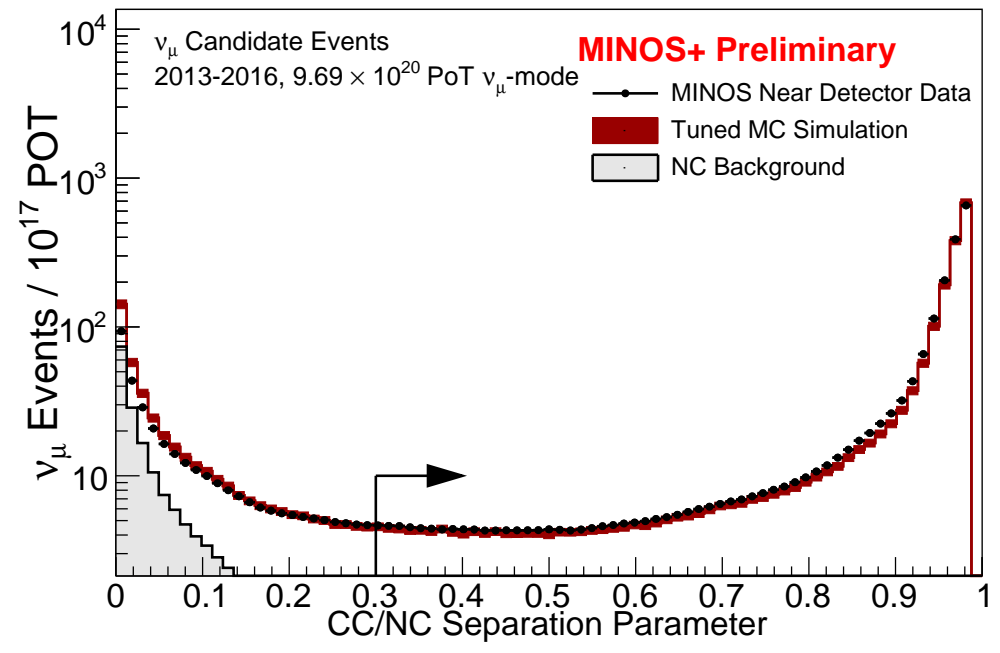

Figure 4.9: MINOS+ $k$ NN ND distribution of the selection parameter for selected $v_{\mu}$-CC events with expected distribution and expected NC contamination. Events with a separation parameter greater than 0.3 are selected.

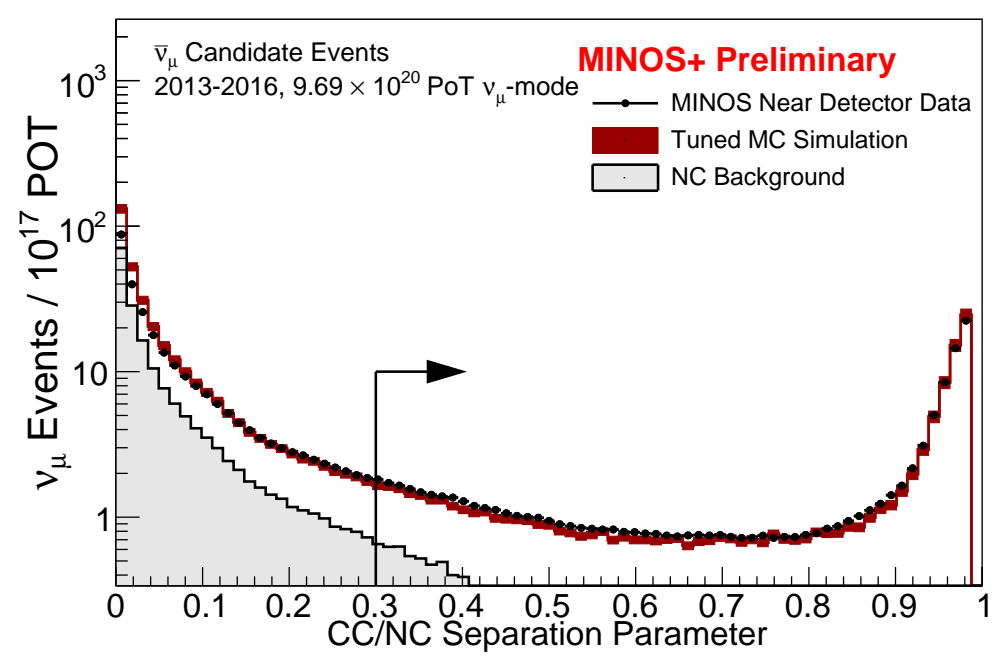

Figure 4.10: MINOS $+k N N$ ND distribution of the selection parameter for selected $\bar{v}_{\mu}$-CC events with expected distribution and expected NC contamination. Events with a separation parameter greater than 0.3 are selected. 

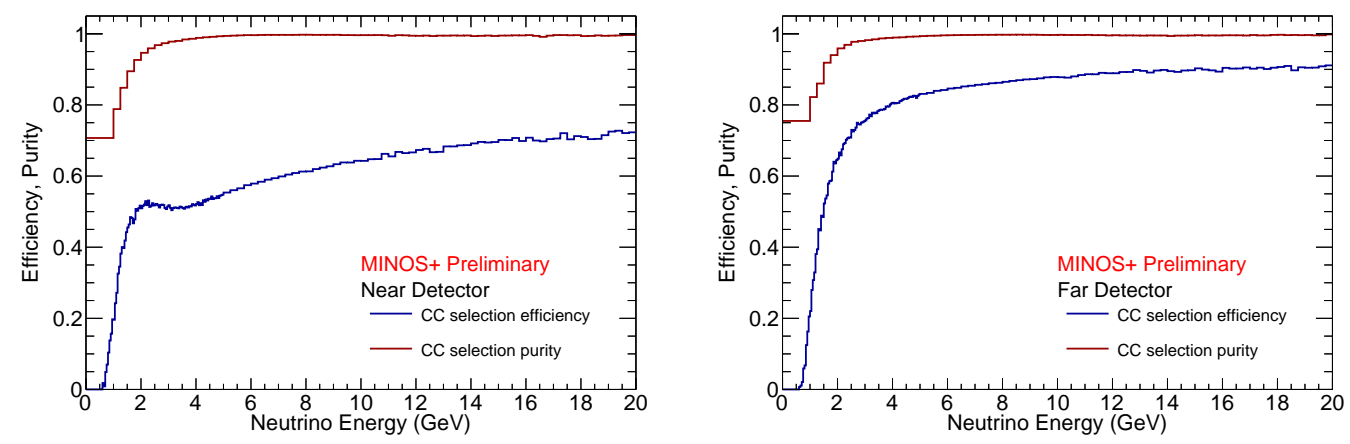

Figure 4.11: MINOS+ CC selection efficiency and purity for $v_{\mu}$ and $\bar{v}_{\mu}$ interactions at the Near Detector (left) and Far Detector (right) as functions of neutrino energy.

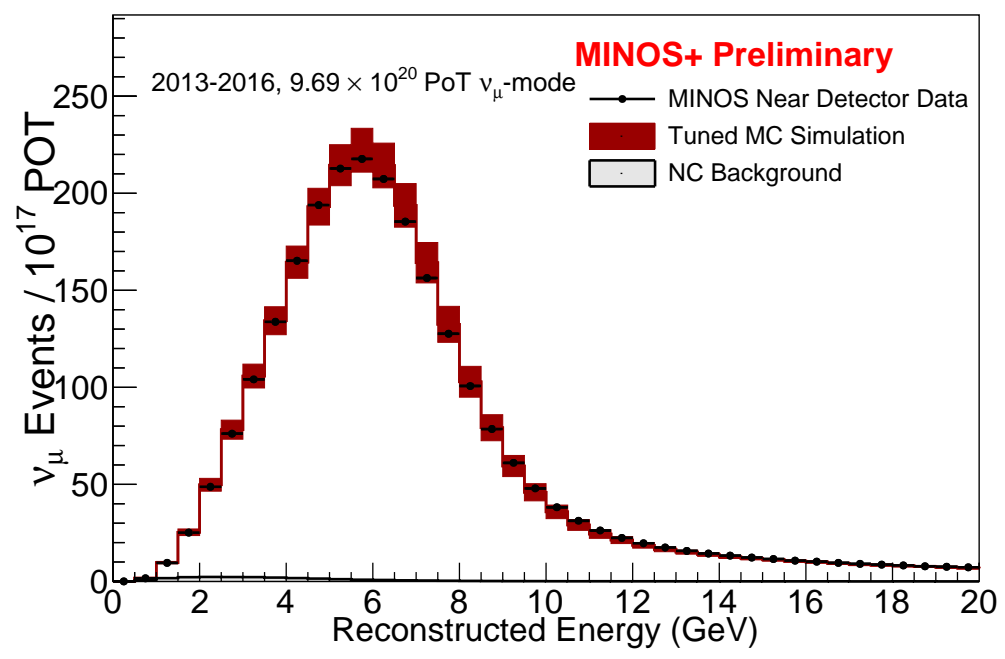

Figure 4.12: MINOS+ selected $v_{\mu}$ and $\bar{v}_{\mu}$ CC spectrum at the Near Detector compared to the MC. 


\subsection{Atmospheric Neutrino Selection}

The details of the atmospheric selection are discussed in Refs. [67, 68]. This section summarizes the event selection requirements to clearly define the atmospheric neutrino samples used in this analysis.

The atmospheric neutrino selection must contend with a large background of cosmic rays. The FD's cosmic-ray veto shield is an important tool for rejecting this background. In addition to occurring outside the NuMI beam spill, atmospheric neutrino candidates must be more than 50 ns removed from the nearest veto shield hit. This requirement eliminates the huge number of cosmic-ray events coincident with veto shield hits, visible as the sharp peak in Fig. 4.13. The additional selection criteria aim to reject cosmic rays that escape the veto shield.

\subsubsection{CV $v_{\mu}$ Events}

The CV selection criteria are designed to reduce the cosmic-ray background incident from above the detector. Cosmic-ray muons that pass the atmospheric neutrino fiducial volume criteria usually enter with a small angle with respect to the detector planes. These cosmic rays are removed by looking at the properties of the track hit closest to the top of the detector, referred to as the upper end of the track.

Cosmic rays entering at steep angles traverse a small distance along the $z$-axis of the detector. To filter out these cosmic rays a quantity called the event trace is defined. The trace is found by first projecting along the 


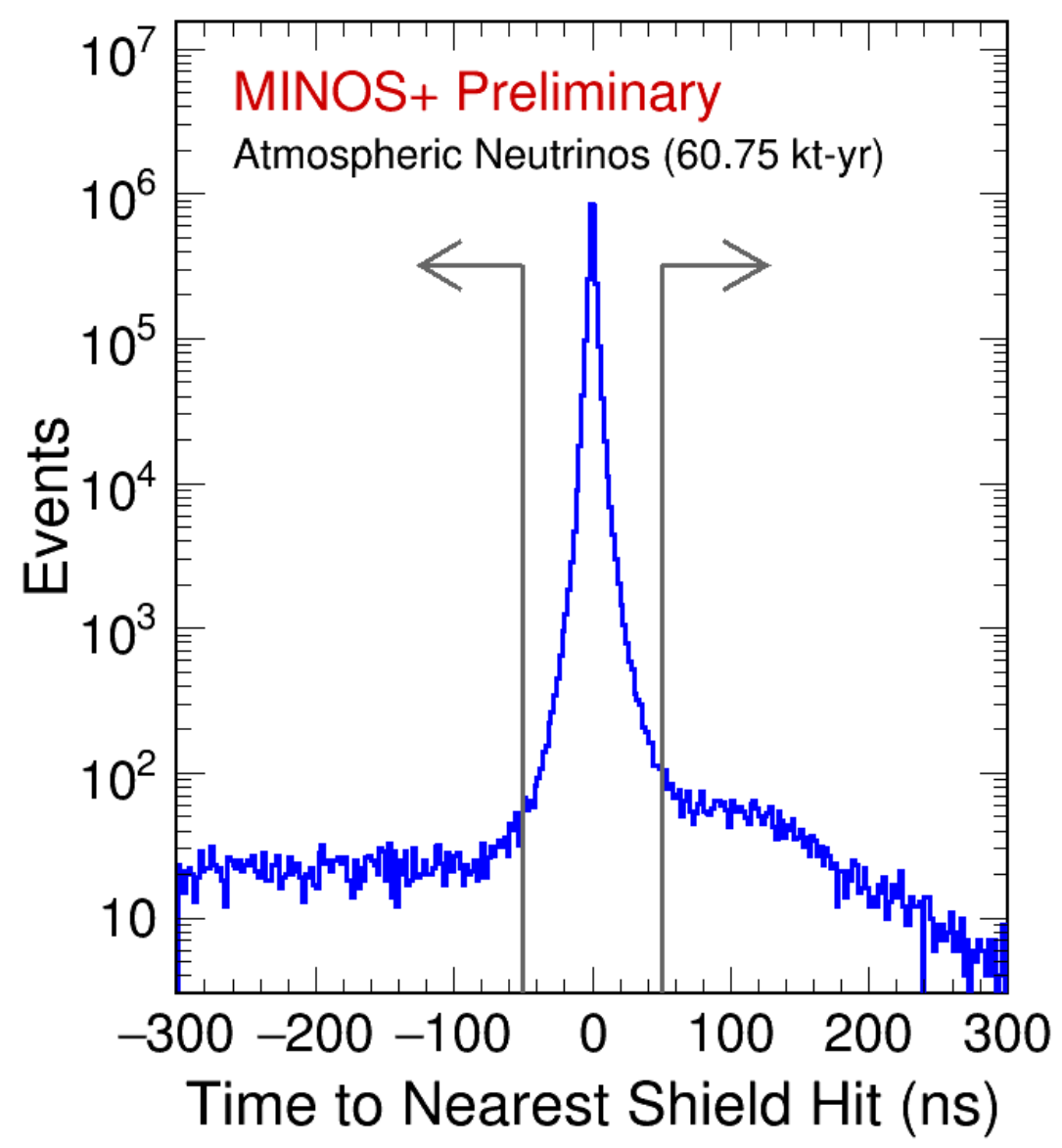

Figure 4.13: The veto shield is used to remove the cosmic-ray muon background in the atmospheric contain-vertex event samples. For each event, the nearest hit in time is located in the sections of the shield above the vertex. The relative time distribution is plotted for a measured cosmic-ray muon sample. The time window of $\pm 50 \mathrm{~ns}$, indicated by the arrows, is used to veto cosmic-ray muons. From Ref. [56]. 
track path to a possible entry point at the top of the detector. The trace, $\Delta_{Z}$, is then the displacement along the $z$-axis between the projected entry point and track hit closest to the top of the detector. Contained vertex events with $\Delta_{Z}>50 \mathrm{~cm}$ are selected. The trace variable is plotted for data and $\mathrm{MC}$ in Fig. 4.14.

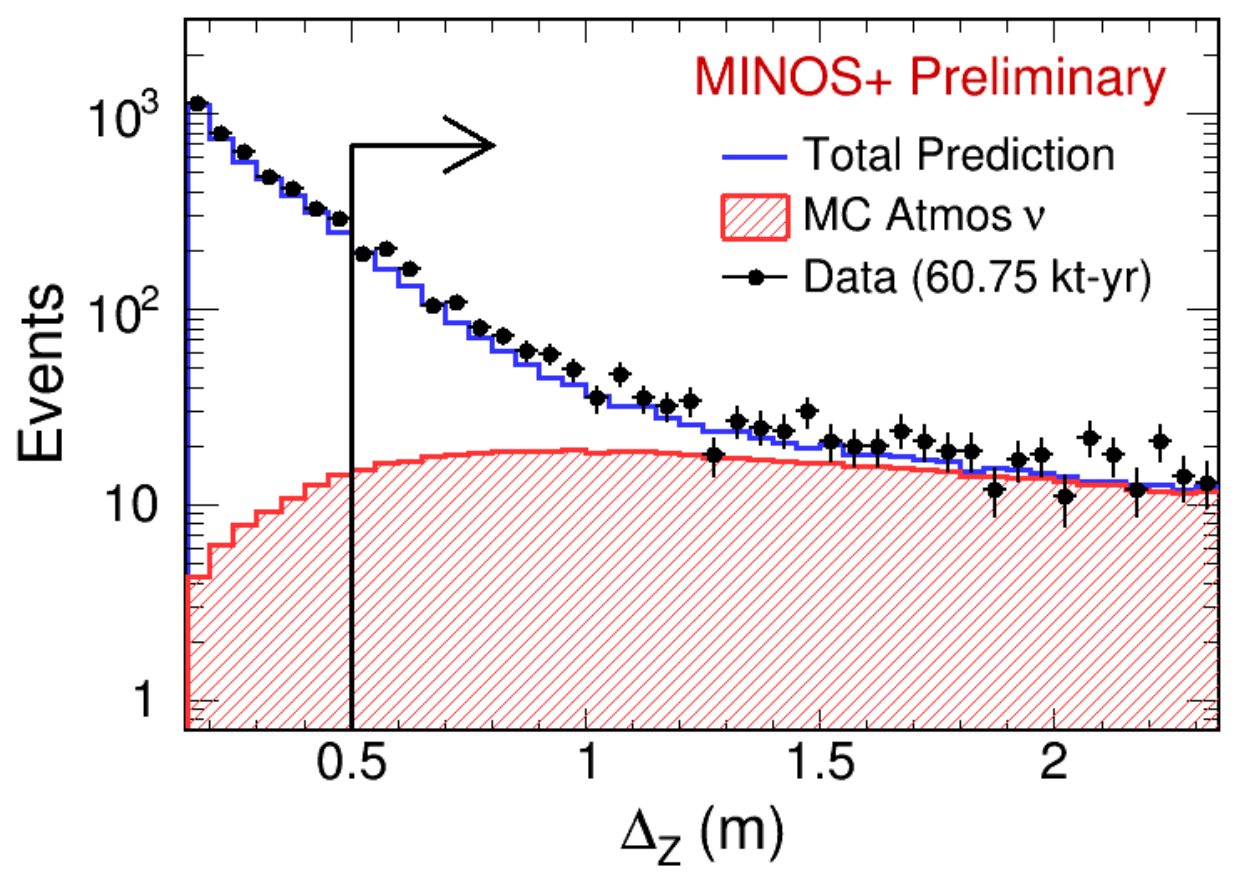

Figure 4.14: Distributions of the trace variable, $\Delta_{Z}$, plotted for contained tracks satisfying the fiducial cuts. The trace is found by extending the track trajectory from the highest vertex back to the edge of the detector and calculating the displacement in $z$. Cosmic-ray muons typically enter the detector at a steep angle and travel a small distance before entering the scintillator. To reject the cosmic-ray muon background, a selection cut of $\Delta_{Z}>0.5 \mathrm{~m}$ is applied to contained-vertex muon tracks. From Ref. [56]. 
In addition to the trace cut, a second requirement is set based on the energy deposition. Cosmic-ray muons that pass the trace cut usually travel through a steel plane before entering the active volume of the detector. As a result, these muons deposit a large amount of energy at the upper end of their tracks. Events that are characteristic of a cosmic ray are rejected by setting a limit on the maximum pulse height, $Q_{\mathrm{vtx}}$, for the upper end of the track. Events with $Q_{\mathrm{vtx}}>300$ photo-electrons (PEs) are rejected. Tracks that are short, crossing fewer than 25 planes, and steep, such that $\cos \theta_{y}>0.7$ or $\left|\cos \theta_{z}\right|<0.5$, are removed if $Q_{\mathrm{vtx}}>75$ PEs. The angles $\theta_{y}$ and $\theta_{z}$ are measured between the path of the upper end of the track and the detector $y$ and $z$ axes. The distributions of $Q_{\mathrm{vtx}}$ for short tracks from simulated neutrinos and cosmicray muons are plotted in Fig. 4.15 with the rejection region highlighted.

\subsubsection{Neutrino Induced Muon Events}

The NIM selection criteria are designed to select muon tracks are traveling upward or horizontally through the detector. Timing information is used to reconstruct the track propagation direction. When the normalized velocity variable, $1 / \beta \equiv 1 /(v / c)$, is plotted before removing downward tracks there is a clear separation between downward tracks $(1 / \beta<0)$ and upward tracks $(1 / \beta>0)$ seen in Fig. 4.16 using only the timing requirement. A small portion of the downward tracks in Fig. 4.16 is attributable to horizontal muons that will survive the final zenith angle requirement. The NIM sample requires that the zenith angle of the track satisfy $\cos \theta_{\text {zen }}<0.05$. The distribution of 

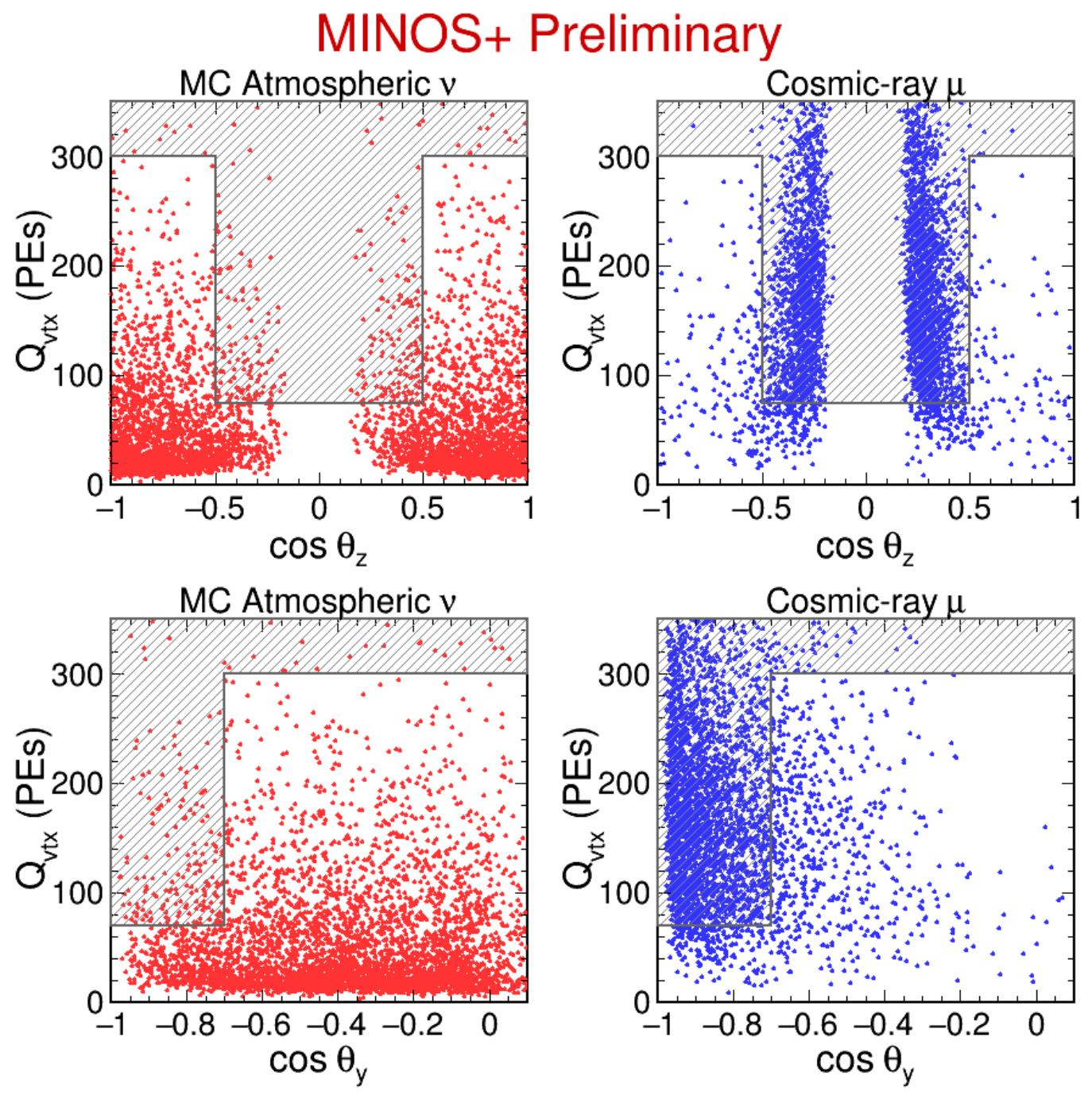

Figure 4.15: Distributions of maximum pulse height at the highest end of the track, $Q_{\mathrm{vtx}}$, plotted against the $y$-component $\left(\cos \theta_{y}\right)$ and $z$-component $\left(\cos \theta_{z}\right)$ of the downward direction. The distributions are plotted for those events that pass the prior trace and topology cuts and span $<25$ planes. The plots on the right show the cosmic-ray muon background, given by vetoed events in data; the plots on the left show Monte Carlo atmospheric neutrinos. The background events are associated with large vertex pulse heights and track directions parallel to the scintillator planes. The hatched regions are rejected by the cuts. From Ref. [56]. 


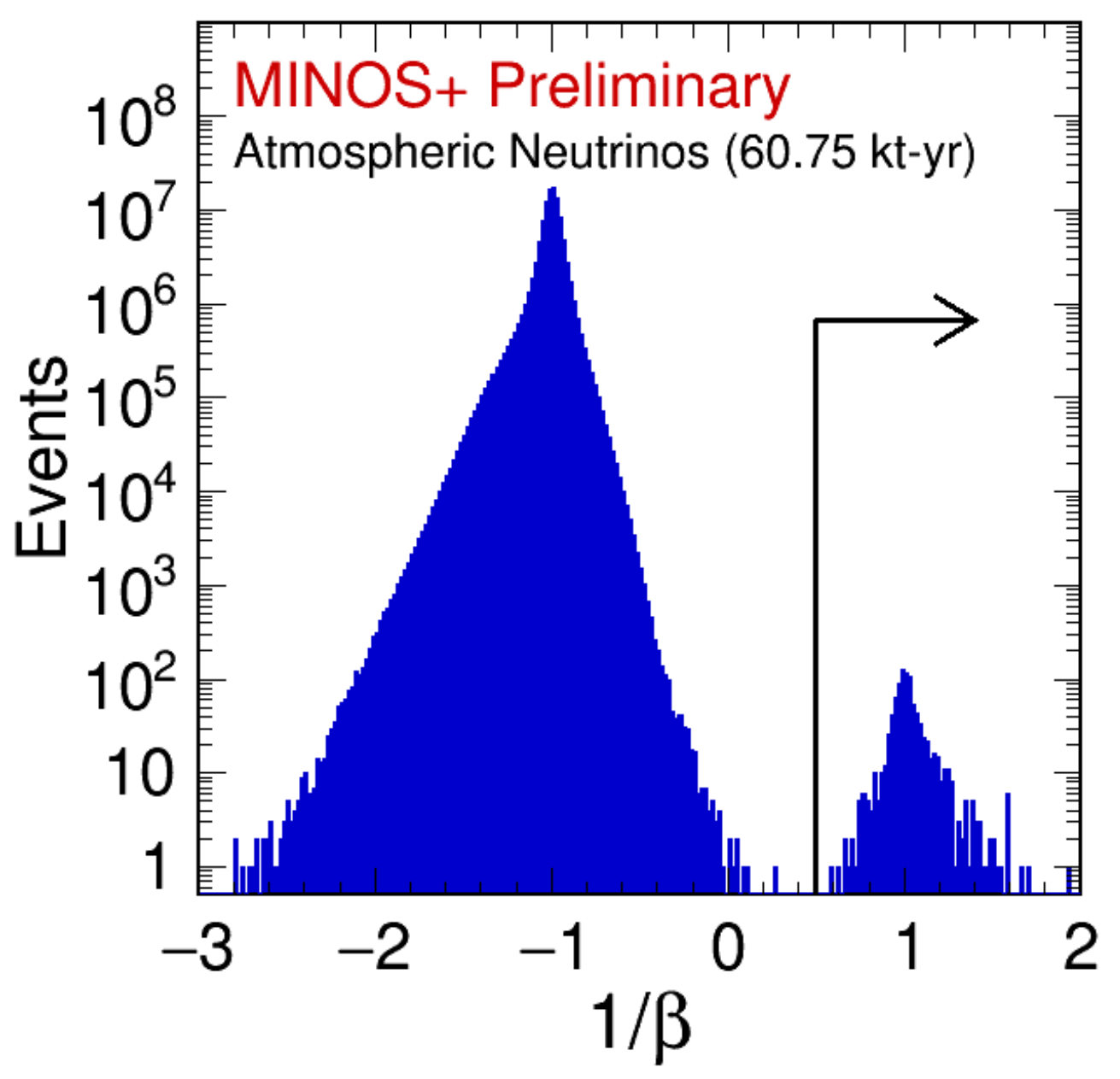

Figure 4.16: Observed distribution of $1 / \beta$ variable, given for each event by the gradient of a linear fit to the measured times as a function of their distance on the track. The distribution is plotted for upward and downward muons that pass the timing cuts. A good separation is achieved between the upward-going neutrino-induced muon signal, which is peaked at +1.0 , and the downwardgoing cosmic muon background, which is peaked at -1.0 . Note that, with a log scale, the peaks appear asymmetric. This is due to the selection cuts, which disfavor $1 / \beta=0$. The arrow indicates upward tracks. From Ref. [56]. 
reconstructed zenith angle for NIM candidate tracks is plotted in Fig. 4.17.

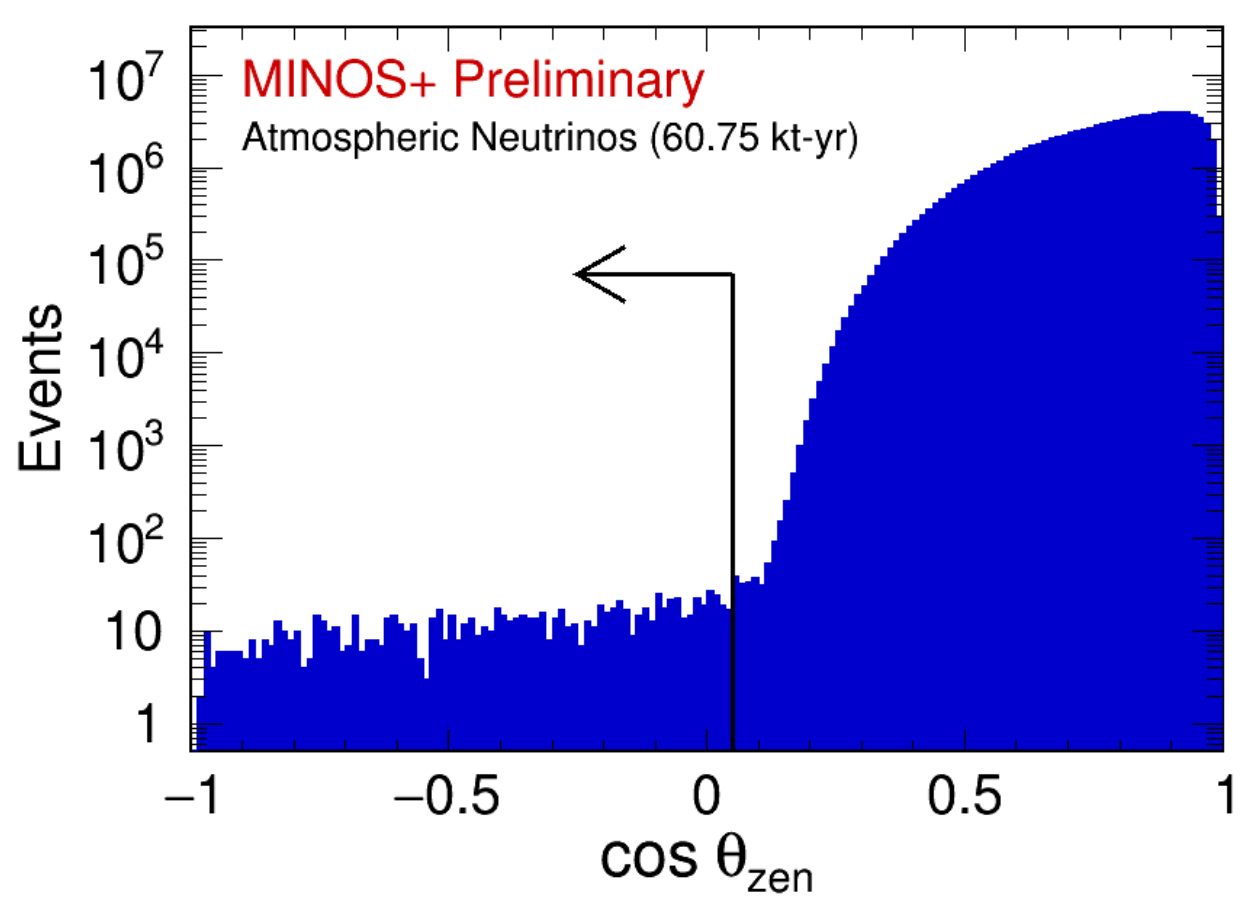

Figure 4.17: Distribution of reconstructed cosine of the zenith angle for muons, with good timing and topology. In the range $0.10<\cos \theta_{\text {zen }}<0.30$, the observed rate of muons is dominated by the cosmic muon background and falls steeply as the rock overburden increases rapidly. For $\cos \theta_{\text {zen }}<0.10$, the distribution flattens, as the cosmic muon flux falls below that of neutrinoinduced muons. To minimize the background from cosmic-ray muons, a cut is placed at $\cos \theta_{\text {zen }}<0.05$. From Ref. [56].

\subsubsection{Shower Events}

Like the CV $\gamma_{\mu}$ selection in Section 4.2.1, the CV shower selection needs to reject background from cosmic rays entering the detector at a steep angle. 
Shower events are required to have $\Delta_{Z}>80 \mathrm{~cm}$, as defined in Section 4.2 .1 with the projection done using the shower vertex. Shower events require a larger trace than $v_{\mu}$ events since the resolution for showers is poorer. The trace of shower event candidates is plotted in Fig. 4.18. Cosmic rays can cause showers in the detector; these showers are characterized by a large amount of deposited energy in a single plane or large energy fluctuations between planes. In contrast, the energy deposition profile of neutrino-induced showers rises to a maximum and smoothly falls. The mean energy deposited per plane, $\left\langle Q_{\mathrm{shw}}\right\rangle$, and the root mean square (RMS) of the energy deposited per plane, $\left\langle Q_{\text {shw }}^{2}\right\rangle^{\frac{1}{2}}$, are used to distinguish between cosmic-ray showers and neutrinoinduced showers. Candidate showers are divided into short showers extending 8 or fewer planes and long showers that extend more than 8 planes. The energy deposited in a plane is measured by the recorded pulse height in units of PEs. Short showers are required to satisfy $\left\langle Q_{\text {shw }}\right\rangle<100 \mathrm{PEs}$ and $\left\langle Q_{\text {shw }}^{2}\right\rangle^{\frac{1}{2}}<100 \mathrm{PEs}$. Long showers are required to satisfy $\left\langle Q_{\text {shw }}\right\rangle<150$ PEs and $\left\langle Q_{\text {shw }}^{2}\right\rangle^{\frac{1}{2}}<150$ PEs. The distributions of $\left\langle Q_{\text {shw }}\right\rangle$ and $\left\langle Q_{\text {shw }}^{2}\right\rangle^{\frac{1}{2}}$ are plotted in Fig. 4.19 for short and long tracks.

\subsection{MINOS Non-Fiducial $v_{\mu}$ Selection}

A special sample of non-fiducial $v_{\mu}$-mode beam events is selected from the MINOS data. The vertices of these events are located outside the fiducial volume, and they are selected using the MINOS $k$ NN algorithms. The selection parameters are relaxed for this sample by lowering the required percentage of 


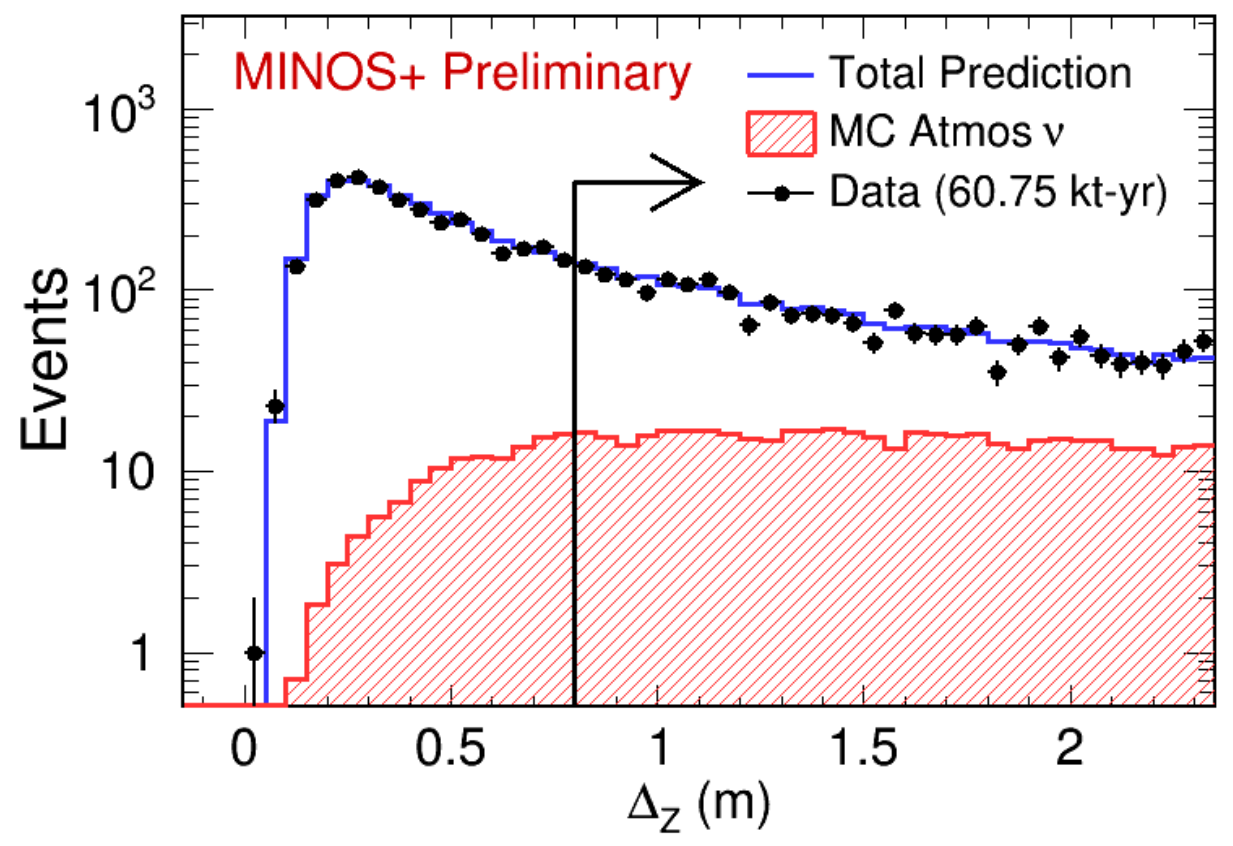

Figure 4.18: Distributions of the trace variable, $\Delta_{Z}$, calculated for containedvertex showers. The hatched histogram shows the Monte Carlo prediction for atmospheric neutrinos. The solid line gives the overall expectation, dominated by cosmic muon background, which is obtained by scaling vetoed events in the data by the measured cosmic-ray veto shield efficiency. Typically, the cosmic-ray muon background events enter the detector at a small angle to the planes and travel a small distance along the $z$ direction before passing into the scintillator. A selection cut of $\Delta_{Z}>0.8 \mathrm{~m}$ is applied to separate signal from background. From Ref. [56]. 

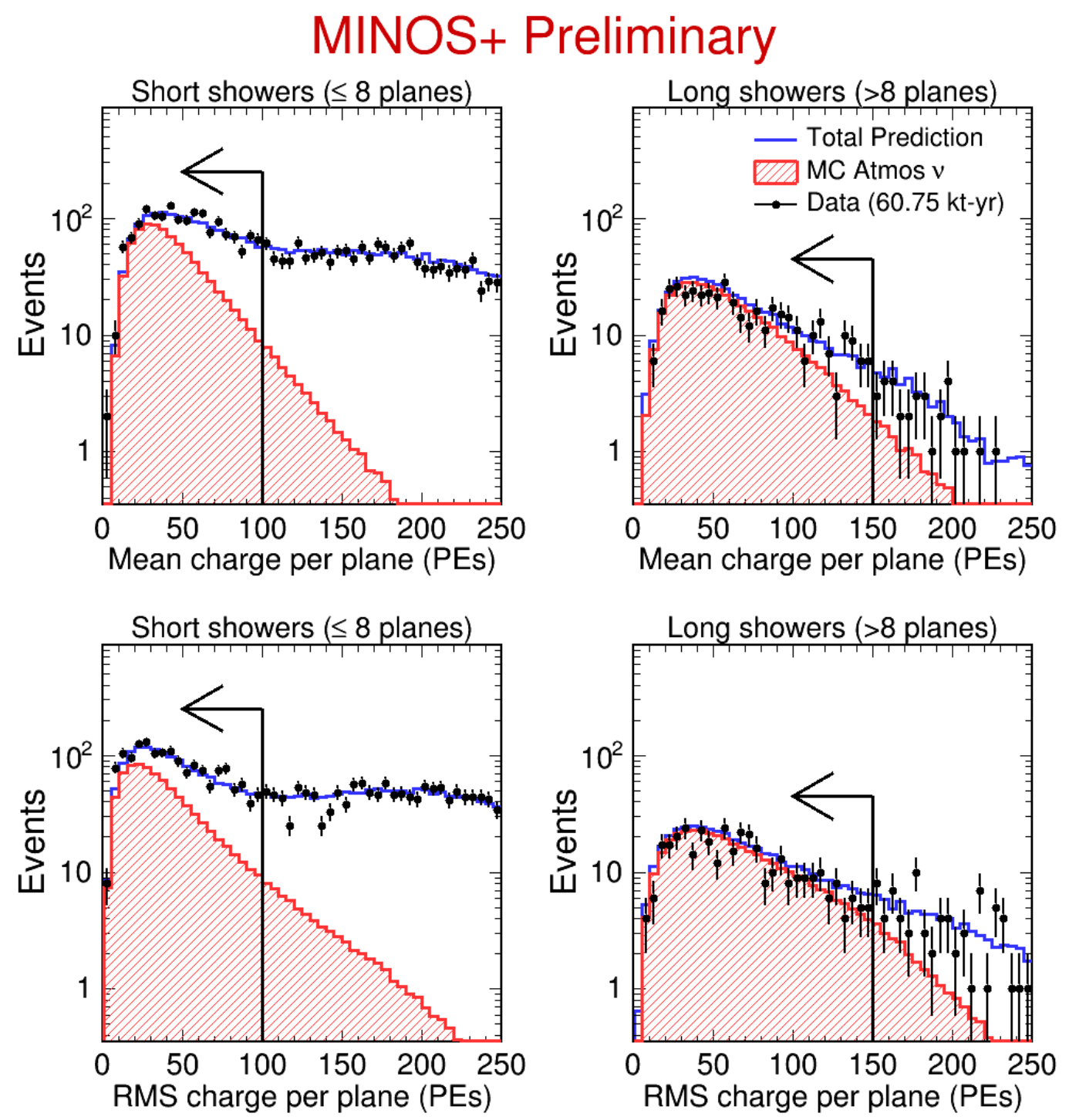

Figure 4.19: Distributions of mean and RMS shower pulse height per plane for contained-vertex showers satisfying the shower trace cuts. Background events from cosmic rays have a larger mean and RMS, particularly for short showers. In order to separate signal and background, events are divided into short $(\leq 8$ planes $)$ and long ( $>8$ planes) showers. The plots show the distributions for each of these samples, and the cuts applied. From Ref. [56]. 
signal events from the nearest neighbors [74].

\subsection{MINOS $v_{\mathrm{e}}$ Selection}

MINOS $v_{\mathrm{e}}$ events are selected using library-event-matching (LEM) $[75,76]$. Each $v_{\mathrm{e}}$ event candidate is compared to a library of simulate signal and background events using the topology of the energy deposition. The 50 best matching simulated events are then used to calculated three selection variables: the fraction of $v_{\mathrm{e}}$-CC interactions, the average inelasticity, and the average fraction of energy depositions that overlap between the test event bestmatching events. These variables are fed into a neural network to discriminate whether the test event is background-like or signal-like. 


\section{Chapter 5}

\section{MINOS+ Neutrino Flux}

For the three-flavor analysis, the measured muon neutrino spectrum at the ND is used to predict the muon neutrino spectrum at the FD. This prediction method is valid since in the case of three-flavor oscillations the neutrino spectrum should be free of oscillation effects at the ND. In order to correctly predict the FD spectrum, the flux calculation must be as accurate as possible. The measured ND spectrum is used to constrain the muon neutrino flux simulation. This method was developed for MINOS due to observed differences between MC and ND data which are shown in Fig. 5.1. The differences occur for all of the MINOS beam configurations; however, the size and energy range of the difference changes with each beam configuration. The fact that the effect is different for each beam configuration suggests that the disagreement is due to inaccurately simulating the neutrino flux as opposed to incorrectly modeling neutrino interactions or the detector [40]. The result of constraining the flux improves the agreement between data and MC at the ND and thus the extrapolation to the FD.

The flux is simulated using FLUGG $[77,78]$. This simulation package uses FLUKA $[79,80]$ to handle the physics simulation and the geometry of the 

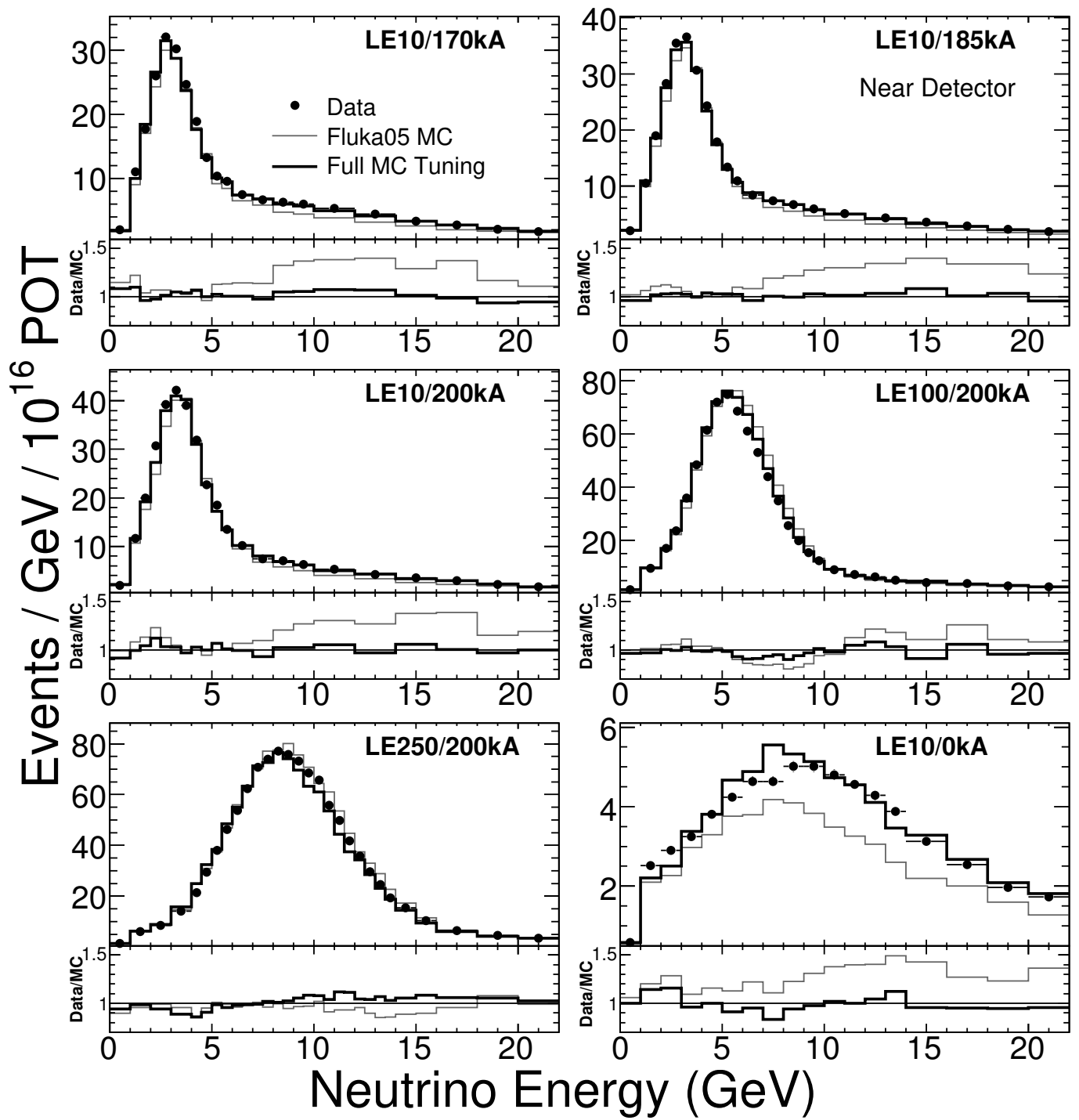

Figure 5.1: The $v_{\mu}$ Near Detector energy spectra for six different beam configurations during the MINOS experiment. The spectra are compared to the default flux simulation using FLUKA05 and the flux simulation tuned using the Near Detector neutrino data. The ratio of the data to MC prediction is shown beneath each spectrum. From Ref. [40]. 
NuMI beamline is defined using GEANT4 [81-83]. The flux simulation has two main sources of uncertainty: hadron production at the target and focusing of the hadron beam. Constraining the neutrino flux proceeds by fitting ND data when the focusing horns were turned off with a function characterizing hadron production at the target. The best fit is then used as a weight to tune the MC. With the hadron production weights applied, the ND data is fit using the dominant focusing uncertainties. The result of the fit is the final focusing weight to tune the MC.

\subsection{Hadron Production}

The hadrons produced by protons colliding with the NuMI target are the neutrino beam source. During normal operations, these hadrons are focused to shape the neutrino beam spectrum for oscillation experiments. The focusing obscures the underlying hadron yields and adds uncertainties from the beam focusing. When the focusing horns are turned off the ND sample the neutrino spectrum from the raw distribution of produced hadrons. This unfocused neutrino spectrum is used to constrain the hadron production at the NuMI target free of focusing effects.

The hadron production uncertainty is constrained with a fit modeled on the work presented in Ref. [41]. In order to do the fit, a function is developed that parameterizes the hadrons produced at the target from the beam simulation. Using this parameterization, the $v_{\mu}$ spectrum at the ND is fit to constrain the $\pi^{+}, \mathrm{K}^{+}$production at the target. The $\pi^{+}, \mathrm{K}^{+}$parameters 
are then fixed, and the $\bar{v}_{\mu}$ spectrum at the ND is fit to constrain the $\pi^{-}$, $\mathrm{K}^{-}$production at the target. Together these fit values determine the hadron production weights for tuning the MC. For this analysis, the hadron production uncertainty constraint was improved by using a new parametrization and accounting for protons that exit the NuMI target. The function

$$
\frac{d^{2} N}{d x_{F} d p_{T}}=\left[B p_{T}+C p_{T}^{2}\right] \exp \left(-D p_{T}^{E}\right)
$$

is used to parameterize the number of hadrons produced off the target for a particular value of $x_{F}$. Examples of best fits for Eq. 5.1 for $\pi^{+}$production for different values of $x_{F}$ are shown in Fig. 5.2. The forms of $B\left(x_{F}\right), C\left(x_{F}\right)$, $D\left(x_{F}\right)$, and $E\left(x_{F}\right)$ are determined empirically by fitting each parameter in bins of $x_{F}$. The fits for the parameters as functions of Feynman's $x_{F}=2 p_{\mathrm{L}} / \sqrt{s}$, where $p_{\mathrm{L}}$ is longitudinal momentum of the meson and $\sqrt{s}$ is the total energy in the center of mass, are shown in Fig. 5.3. 

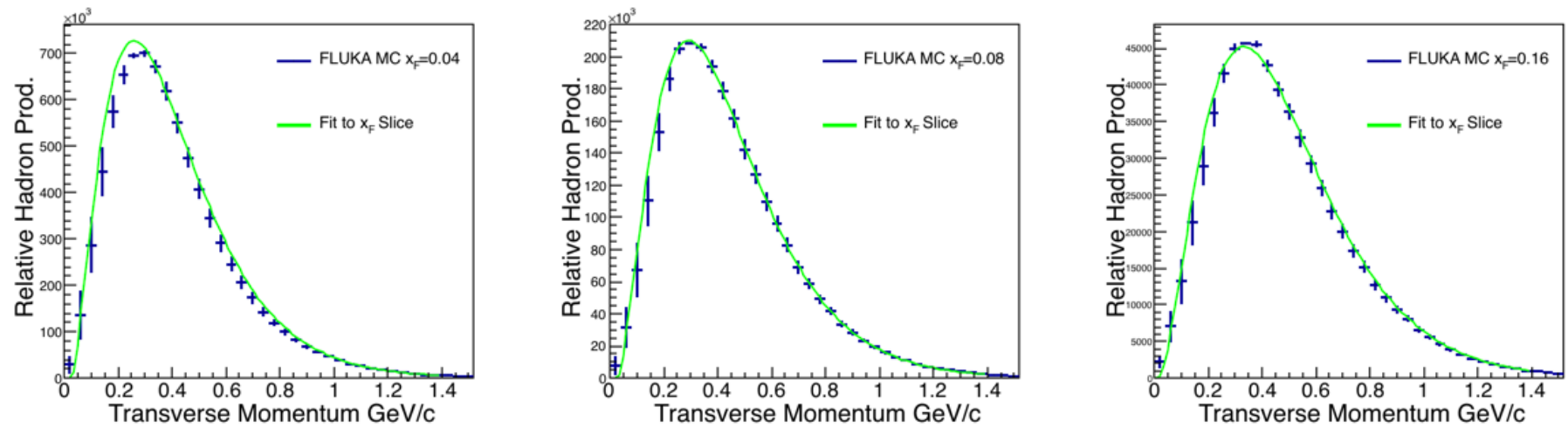

ठे
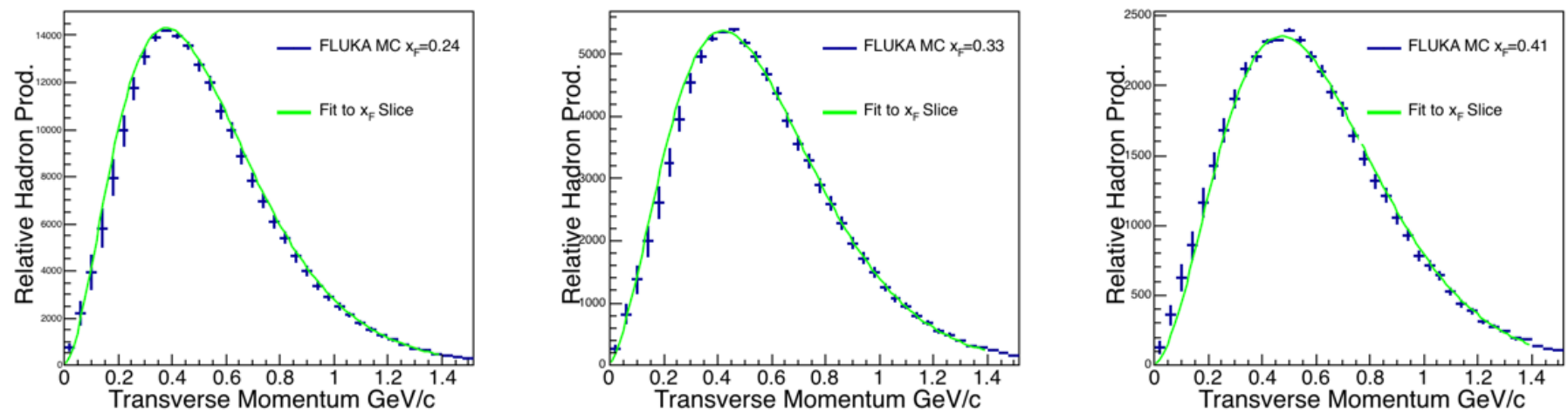

Figure 5.2: Production yields for $\pi^{+}$as functions of transverse momentum for different values of $x_{F}$. Courtesy of Anna Holin. 

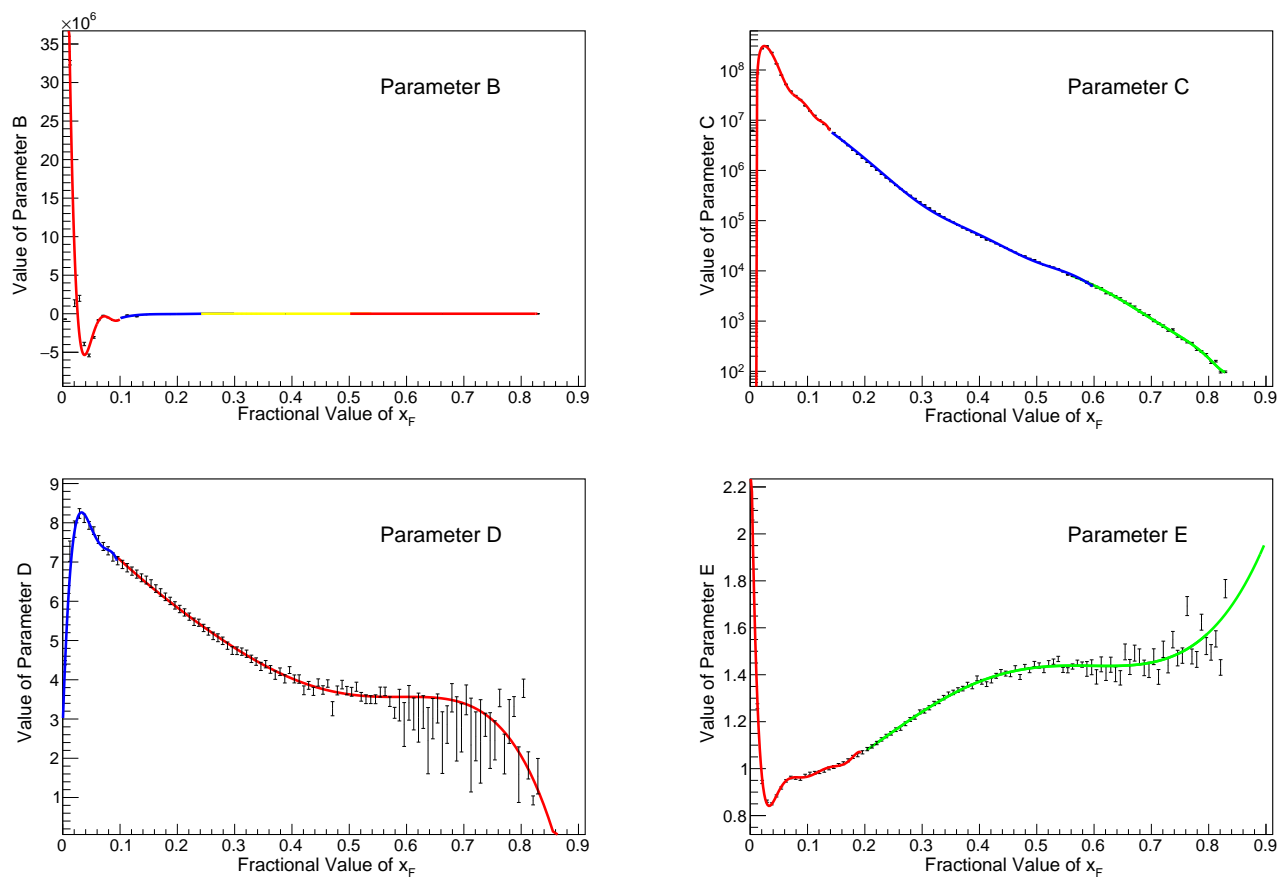

Figure 5.3: The best fits for $B\left(x_{F}\right), C\left(x_{F}\right), D\left(x_{F}\right)$, and $E\left(x_{F}\right)$ for $\pi^{+}$. From Ref. [84].

The fit to the data optimizes 8 parameters which modify the functions $B\left(x_{F}\right), C\left(x_{F}\right), D\left(x_{F}\right)$, and $E\left(x_{F}\right)$ in the following way:

$$
\begin{aligned}
& B^{\prime}\left(x_{F}\right)=\left(p_{0}+p_{1} x_{F}\right) B\left(x_{F}\right) \\
& C^{\prime}\left(x_{F}\right)=\left(p_{2}+p_{3} x_{F}\right) C\left(x_{F}\right) \\
& D^{\prime}\left(x_{F}\right)=\left(p_{4}+p_{5} x_{F}\right) D\left(x_{F}\right) \\
& E^{\prime}\left(x_{F}\right)=\left(p_{6}+p_{7} x_{F}\right) E\left(x_{F}\right)
\end{aligned}
$$

The hadron yields are then scaled by the function

$$
W\left(p_{T}, x_{F}\right)=\frac{\left[B^{\prime} p_{T}+C^{\prime} p_{T}^{2}\right] \exp \left(-D^{\prime} p_{T}^{E^{\prime}}\right)}{\left[B p_{T}+C p_{T}^{2}\right] \exp \left(-D p_{T}^{E}\right)} .
$$


In the MINOS+ parameterization, $\mathrm{K}^{+}\left(\mathrm{K}^{-}\right)$mesons get the same weights as $\pi^{+}\left(\pi^{-}\right)$meson since it has been demonstrated that FLUKA correctly prediction the ratio of pion kaon production for proton carbon interactions [85]. The resulting $\pi^{+}$weights are shown in Fig. 5.4.

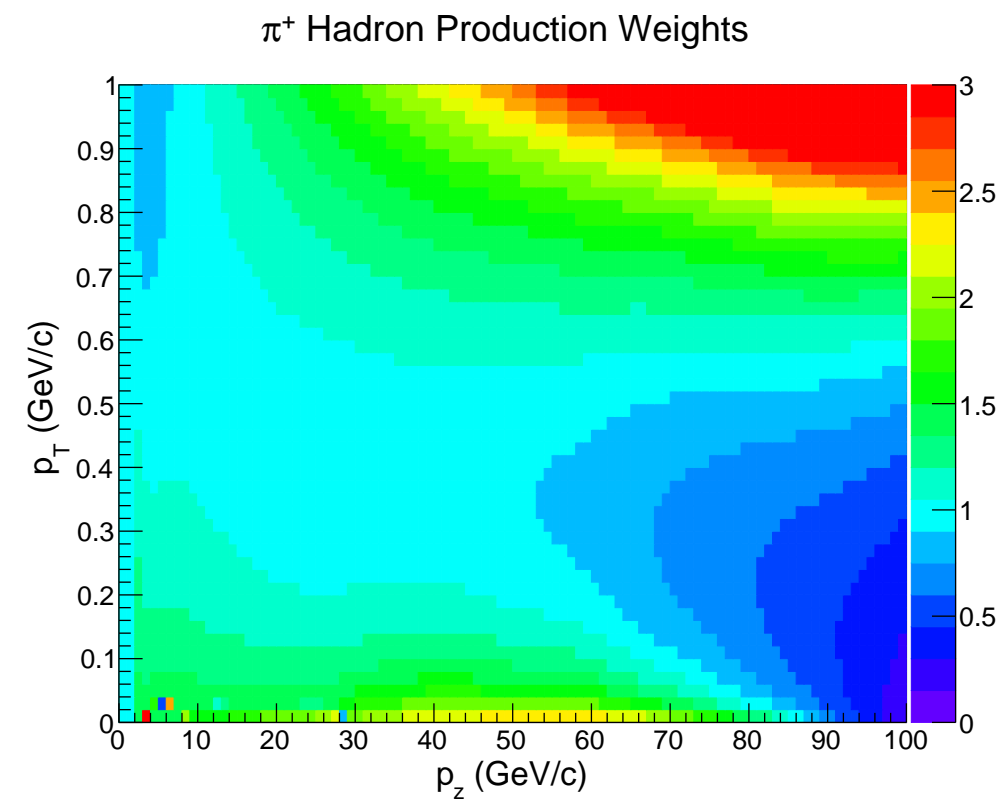

Figure 5.4: The $\pi^{+}$hadron production weights after fitting the horn off ND data. From Ref. [84].

The result of applying the hadron production weight to the $\mathrm{MC}$ is shown in Fig. 5.5.

\section{$5.2 \quad$ Focusing}

The weights from fitting the hadron prodcution using horn off data can then be applied to MC for when the horns are on as in Fig. 5.6. After 


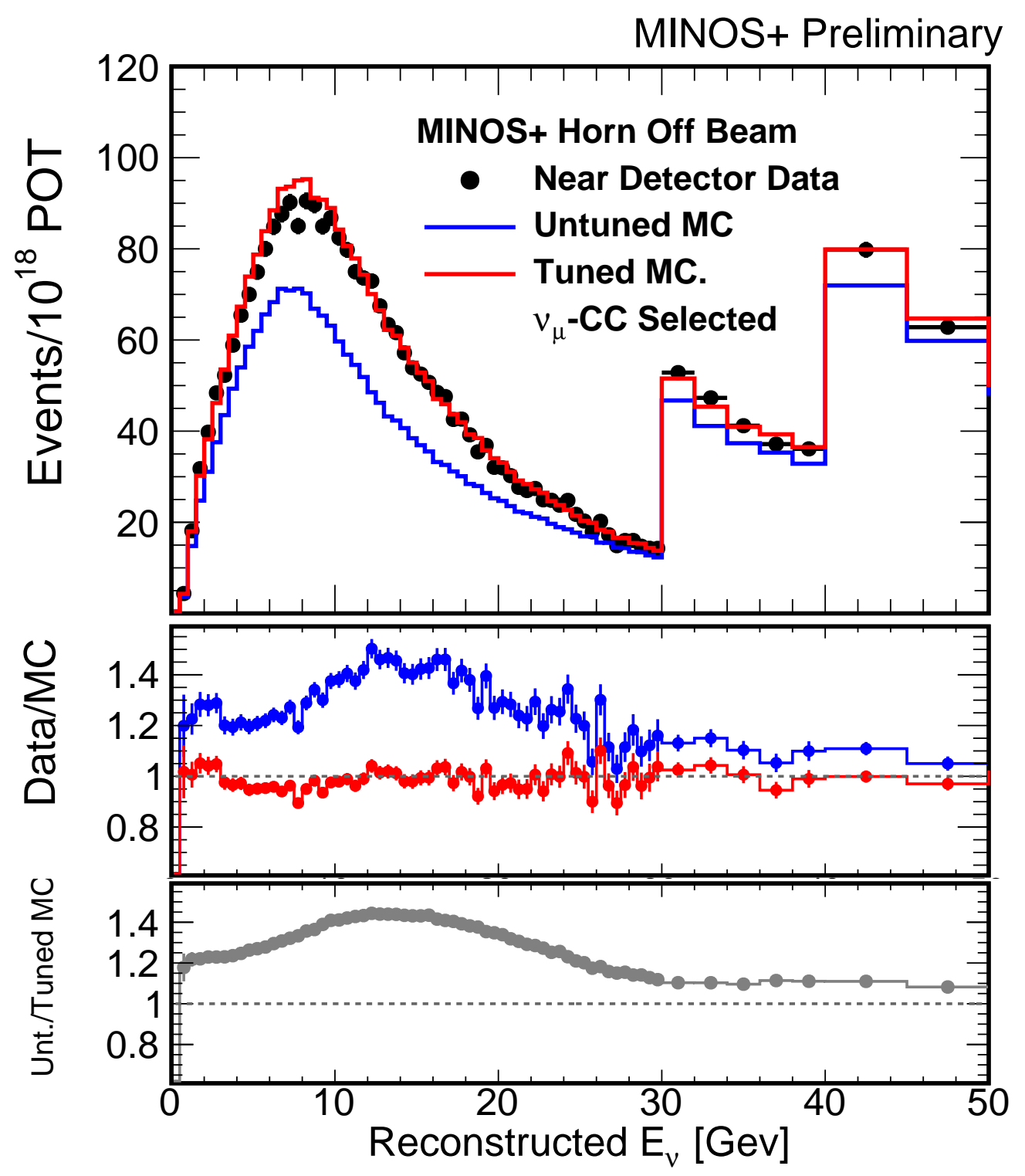

Figure 5.5: The measured ND horn off $v_{\mu} \mathrm{CC}$ spectrum compared to the the $\mathrm{MC}$ before (blue) and after (red) the hadron production fit. From Ref. [84]. 
applying the weights, there remains a significant data/MC difference just above the focusing peak energy. This difference is attributed to uncertainties on the beam focusing.

The focusing uncertainties concern the properties of the NuMI proton beam and focusing horns. These uncertainties are evaluated by comparing simulated fluxes with the properties modified from the design values. The ratio of the change in the flux to the nominal flux defines the error band for the uncertainty. These bands are calculated for the ND and FD fluxes. Another band is calculated on the ratio of the FD to ND flux. This Far/Near band estimates the uncertainty on extrapolating the ND flux to the FD. The uncertainties that best account for the difference between ND data and MC are used to tune the MC to agreement.

\subsubsection{Focusing Systematic Uncertainties Horn Current}

The horn current miscalibration uncertainty quantifies the uncertainty on the current delivered to the focusing horns. The horns are pulsed from a bank of capacitors. The delivered current is monitored using current transformers. Calibration measurements of the system find an error on the horn current within $0.5 \%[86,87]$. Previous MINOS+ three-flavor analyses used an uncertainty of $1 \mathrm{kA}$, which translates to $0.5 \%$ of the nominal $200 \mathrm{kA}$. However, there is concern that this uncertainty has been underestimated, and fits to the focused ND spectrum prefer a horn current $10 \mathrm{kA}$ lower than the nomi- 


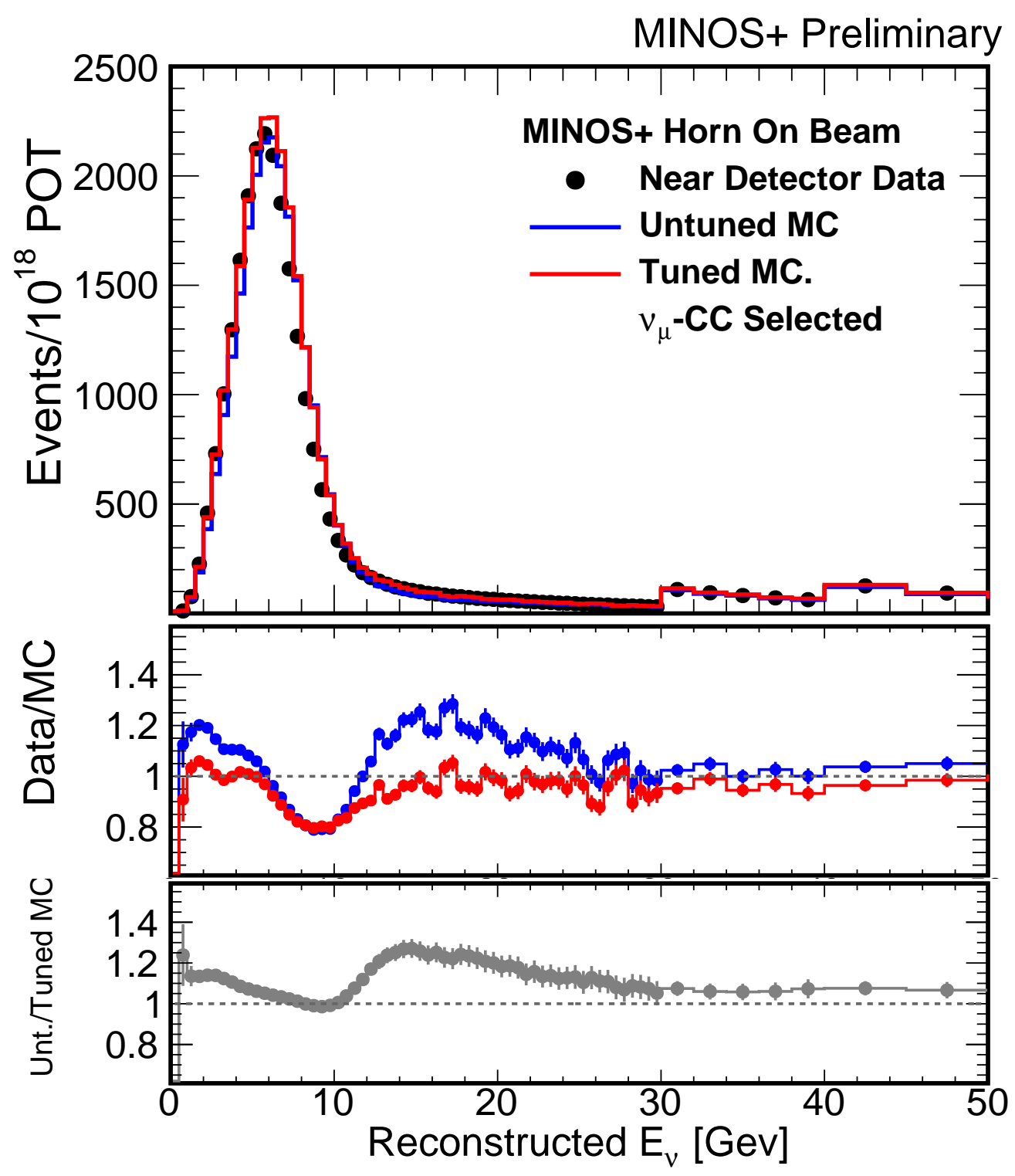

Figure 5.6: The measured ND horn on $v_{\mu} \mathrm{CC}$ spectrum compared to the the $\mathrm{MC}$ before (blue) and after (red) the hadron production fit. From Ref. [84]. 
nal value. For this analysis, the uncertainty is set to a conservative $10 \mathrm{kA}, 5 \%$ of the nominal $200 \mathrm{kA}$ for the MINOS+ beam.

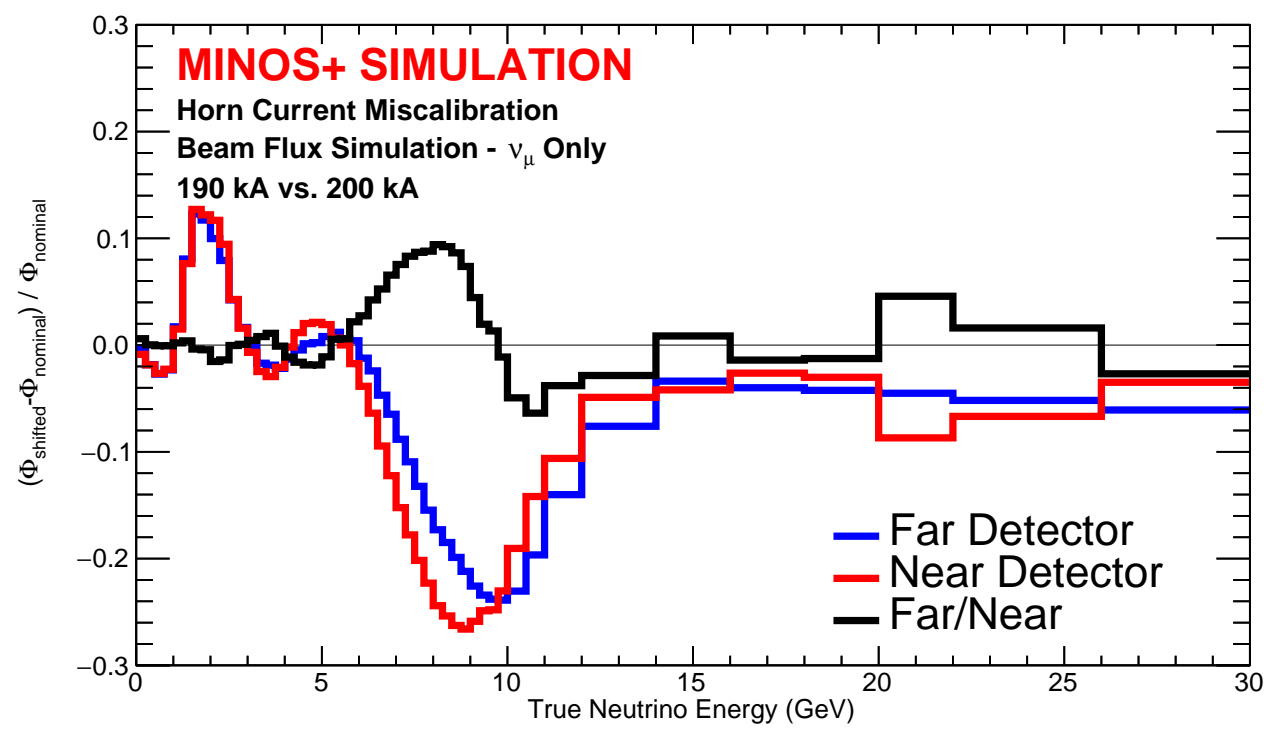

Figure 5.7: The 10kA horn current miscalibration focusing uncertainty for muon neutrinos on the nominal 200kA horn current.

\section{Material Error}

The material error uncertainty attempts to quantify uncertainties in the amount of material in the beamline particularly for the focusing horns. For instance, the flux simulation neglects the layer of water that is sprayed on the focusing horns for cooling [88]. The uncertainty is evaluated as $5 \%$ of the ratio of the flux with the horns replaced with air to the nominal setup using aluminum horns. 


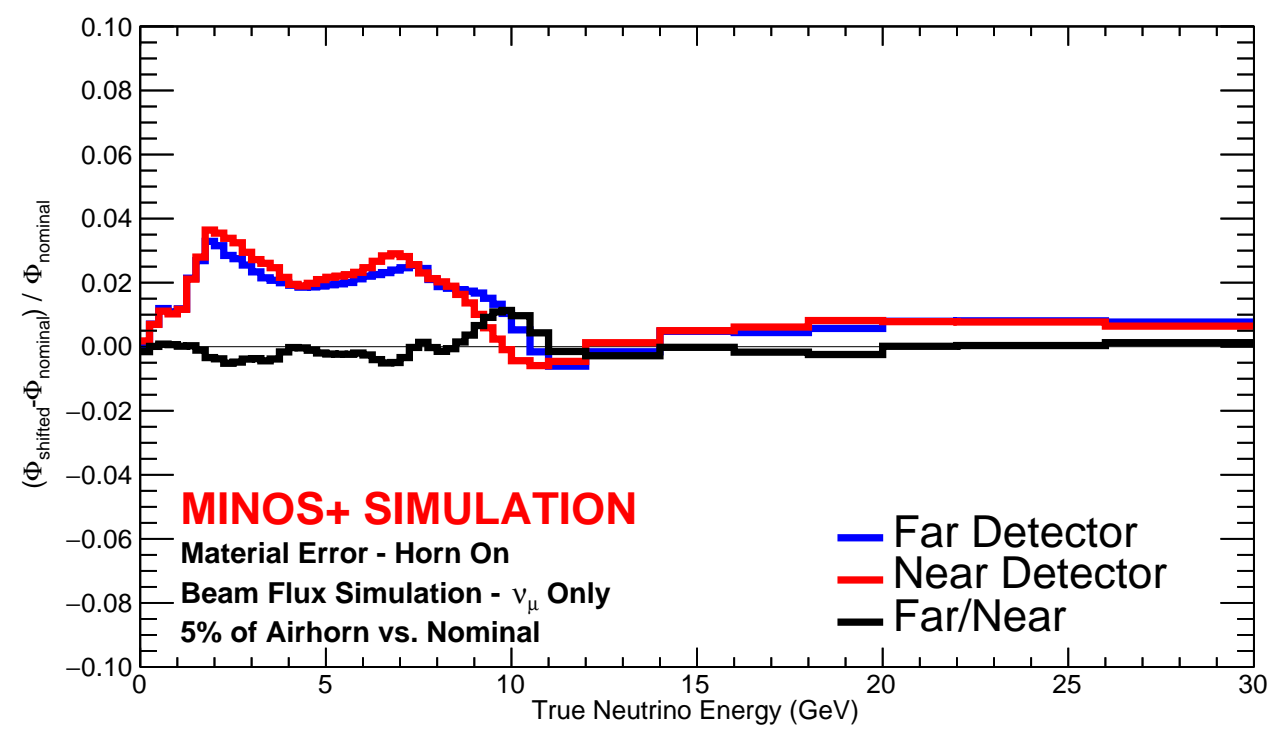

Figure 5.8: Horn material uncertainty for muon neutrinos calculated as $5 \%$ of the result of replace the horns in the flux simulation with air.

\section{Horn Current Distribution}

If the focusing horns were ideal conductors, all of the current would flow on the surface of the horns. It is more realistic to expect the current to be distributed beneath the surface. As a result, particles traveling through the aluminum are sensitive to the effect of the horn current distribution on the magnetic field. The neutrino flux simulation assumes an exponential distribution of the horn current with a skin depth $\delta=7.7 \mathrm{~cm}$. The uncertainty is evaluated by taking the ratio of the flux assuming the current is uniformly distributed throughout the conductor $(\delta=\infty)$ to the nominal flux simulation. 


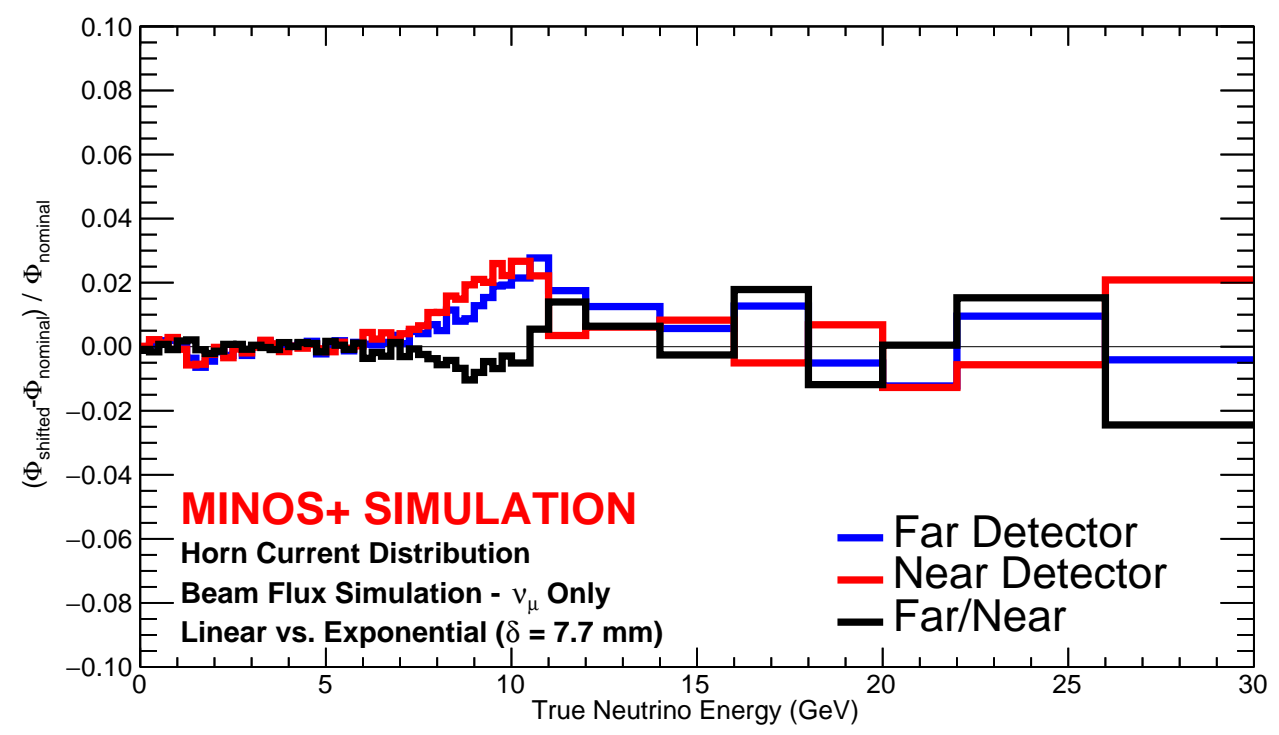

Figure 5.9: Horn current distribution uncertainty for muon neutrinos.

\section{Horn 1 Misalignment}

The uncertainty on the alignment of Horn 1 is evaluated by comparing the flux resulting from shifting the horn horizontally or vertically by $\pm 0.5 \mathrm{~mm}$ to the nominal flux. Shifting the horn by $-0.5 \mathrm{~mm}$ in the vertical direction resulted in the largest difference and was chosen as the systematic uncertainty.

\section{Beam Position}

The uncertainty on the beam's position on the face of the target is evaluated in the same manner as the Horn 1 misalignment uncertainty. A $0.5 \mathrm{~mm}$ in the vertical direction resulted in the largest change from the nominal 


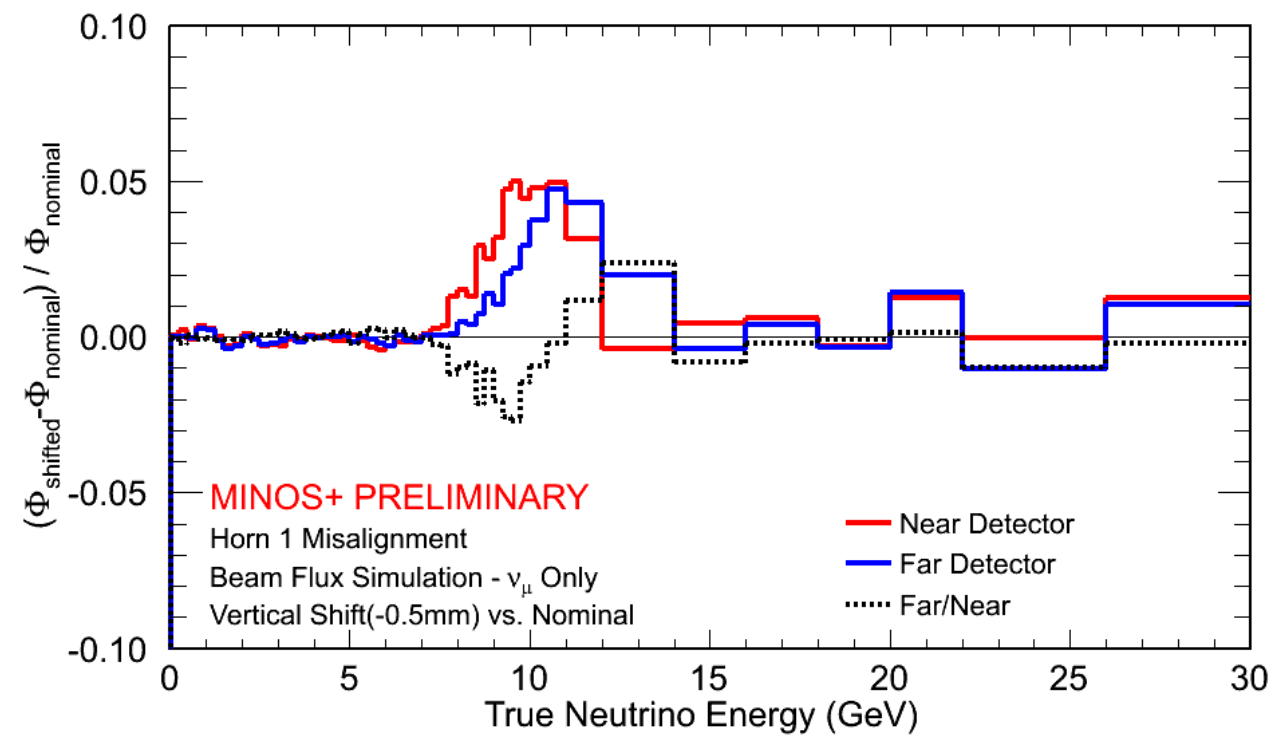

Figure 5.10: Horn 1 alignment uncertainty for muon neutrinos. Plot from Ref. [89].

flux. This was a new focusing systematic that was not evaluated for the MINOS beam [89].

\section{Beam Width}

The proton beam width systematic quantifies the uncertainty in the profile of the proton beam hitting the target. The size of the beam spot changes during running particular as the beam intensity fluctuates. As the intensity increases so does the proton beam width. The uncertainty on the beam width is taken to be $0.2 \mathrm{~mm}$. 


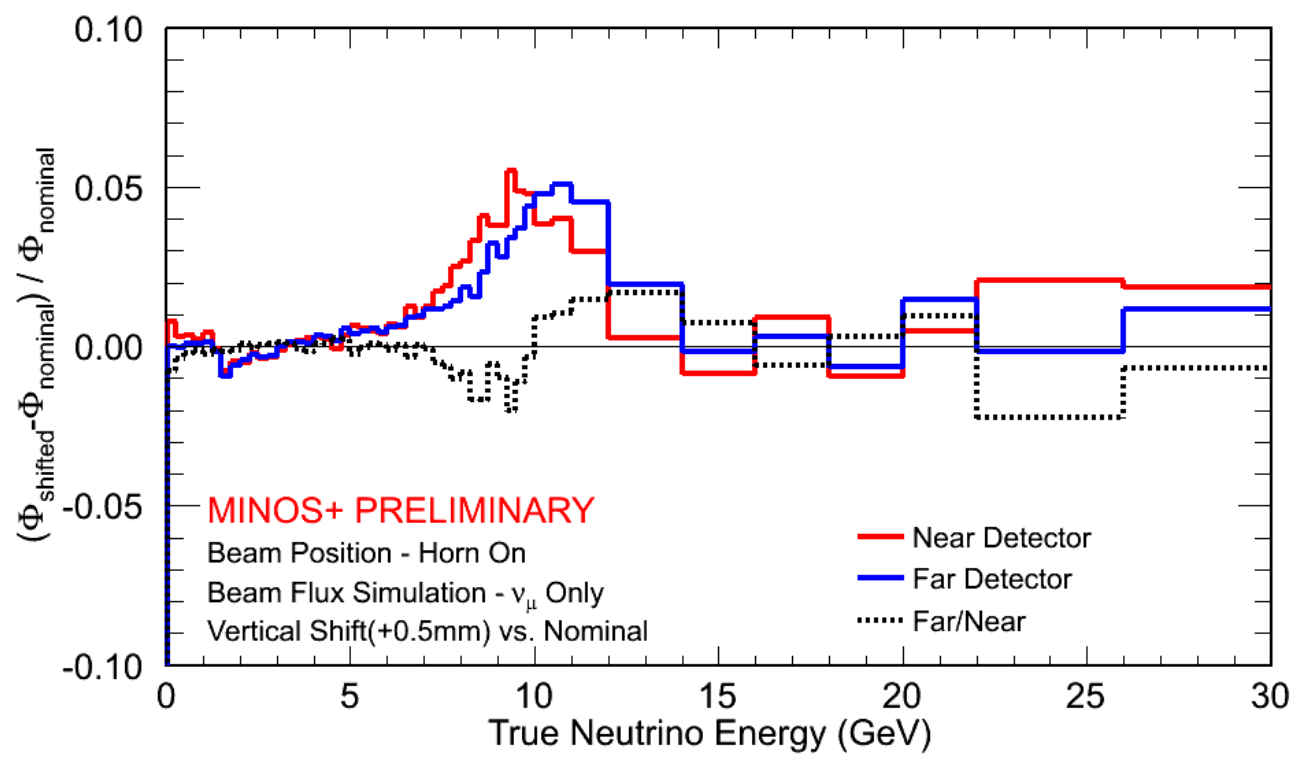

Figure 5.11: Proton beam position uncertainty for muon neutrinos. Plot from Ref. [89].

\section{Number of Protons on Target}

There is an uncertainty on the total number of protons hitting the target. The error stems from uncertainties on counting how many protons make it to the target as well as how many protons actually hit the target. The uncertainty was set at a $2 \%$ normalization uncertainty [41].

\section{Baffle Scraping}

The baffle scraping uncertainty accounts for modifications to the neutrino flux from the proton beam interacting with the baffle described in Section 2.1. For MINOS, the amount of baffle scrapping was estimated by mea- 


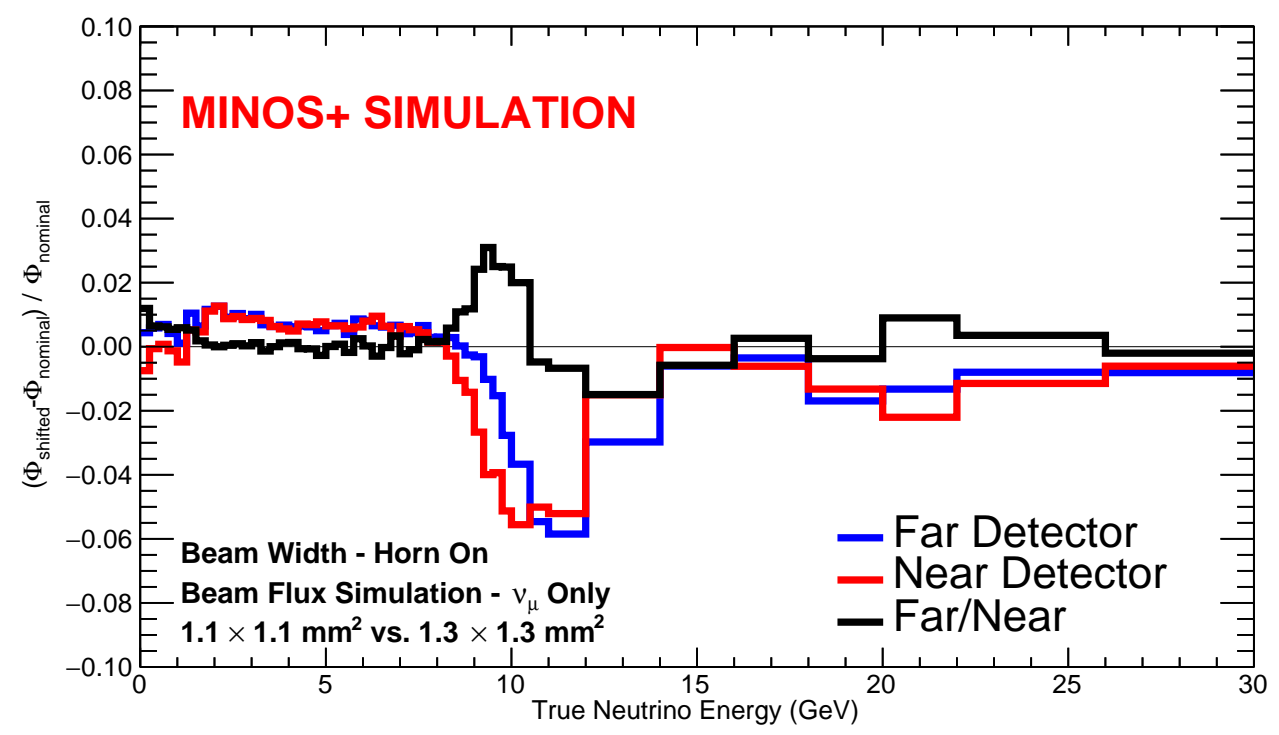

Figure 5.12: Proton beam width uncertainty for muon neutrinos.

suring the change in temperature of the baffle during beam operation [41]. The flux from baffle scraping was simulated by constructing a linear combination the flux from the entire proton beam hitting the baffle and the flux when all the protons hit the target [90]. The final uncertainty was the ratio of the linear combination to the nominal flux. This technique is believed to have overestimated the effect of baffle scraping effect [91]. For MINOS+, the uncertainty was evaluated by increasing the beam spot size by $1 \sigma$ and comparing the flux when interactions in the baffle are tracked to when interactions in the baffle are ignored $[91,92]$. This comparison concluded that the effect is negligible. 


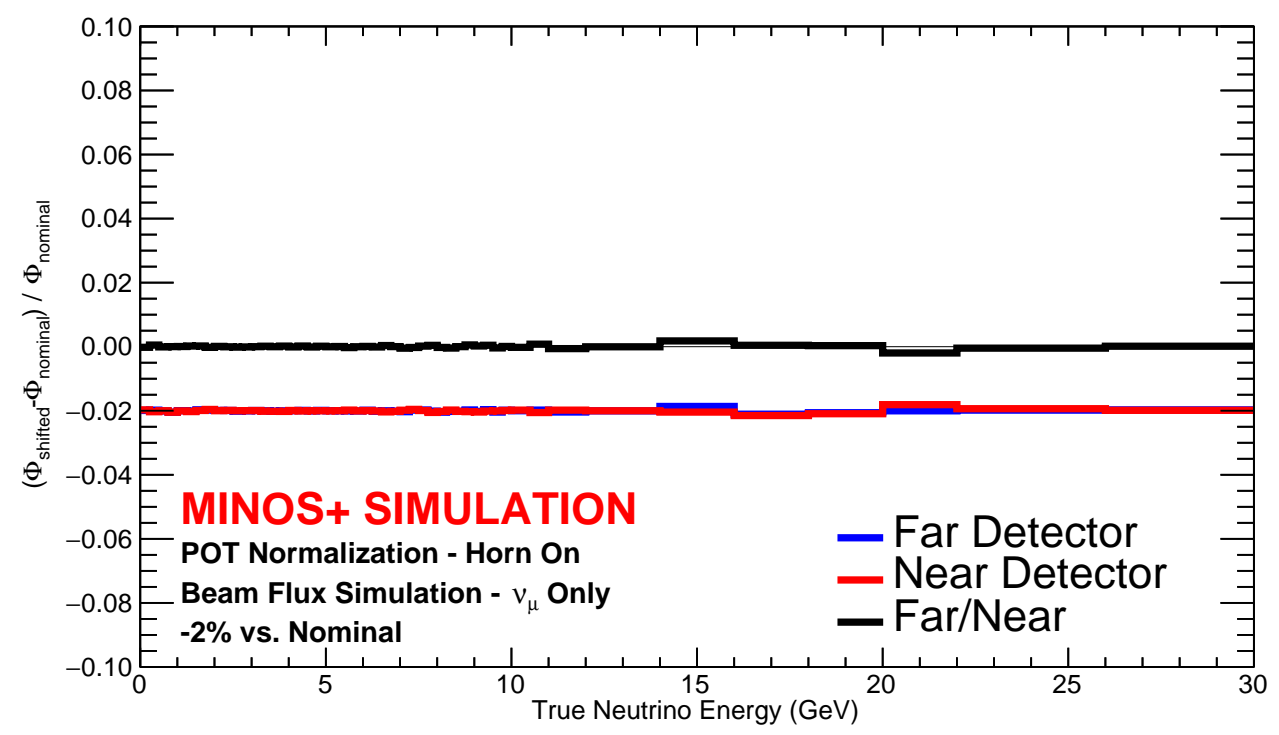

Figure 5.13: Number of protons on target uncertainty for muon neutrinos.

\subsubsection{Summary of Focusing Systematics}

Figures 5.7 to 5.14 show the calculated focusing uncertainties on the flux. The focusing systematics and their uncertainties are summarized in Table 5.1. These uncertainties are used when comparing the measured ND spectrum to MC.

\subsubsection{Focusing Fit}

The focusing uncertainties used to fit the MINOS+ ND spectrum are the horn current and horn material uncertainties. These uncertainties best accounted for the differences between data and MC. The MC with hadron production weights applied is used to fit the ND data allowing the MC to vary 


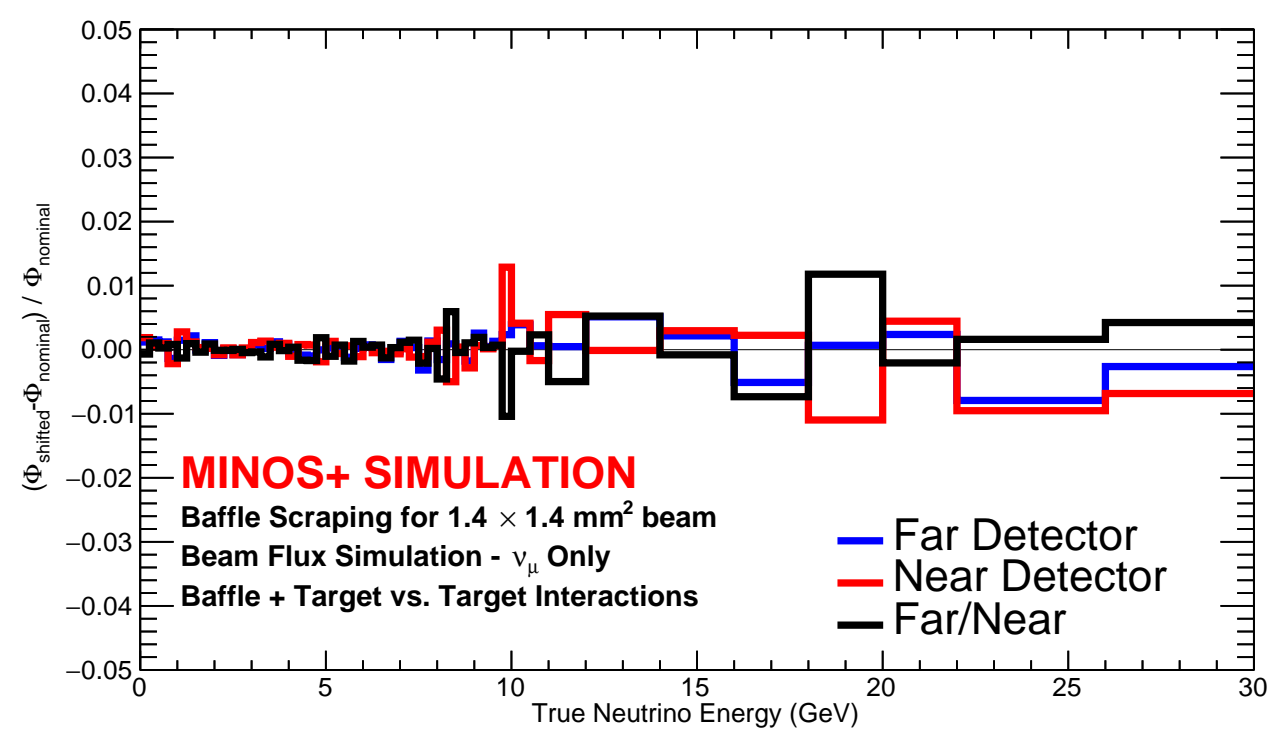

Figure 5.14: Baffle scraping uncertainty for muon neutrinos.

within the two systematic uncertainties. The final MC used in the analysis for predicting the muon neutrino spectrum at the FD has both the hadron production weights and the focusing weights applied. The result of applying the weights is plotted in Fig. 5.15.

\subsection{Run 13 Horn Tilt}

On October 30, 2016 NuMI operators discovered that Horn 1 was out of position while doing beam-based alignment of the neutrino beam components $[93,94]$. An investigation found that a bushing, keeping the upstream end of the horn in place, had failed allowing that end of the horn to drop about $4 \mathrm{~mm}$ [93-96]. This drop is equivalent to the horn pivoting on the horn's 


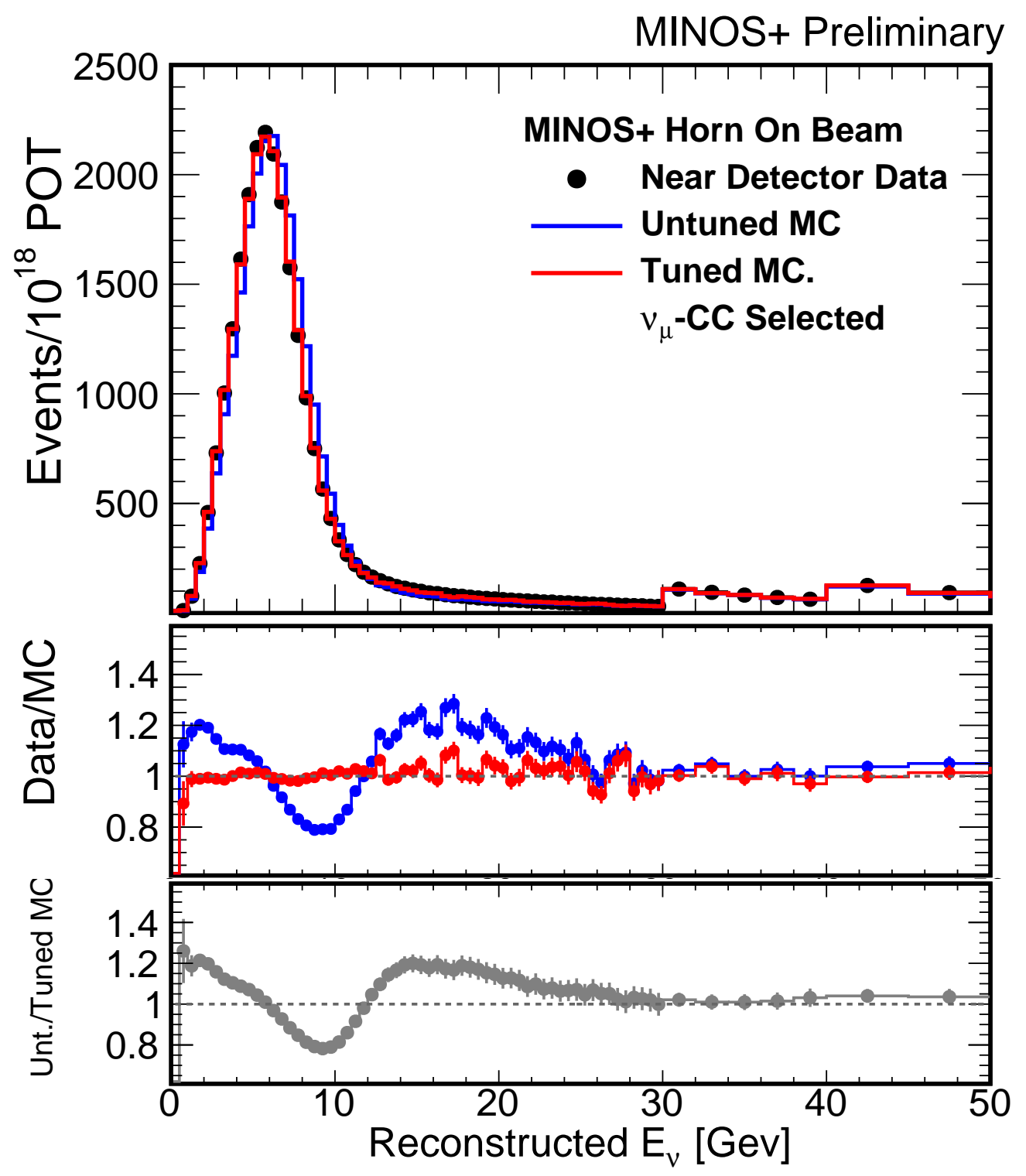

Figure 5.15: The measured ND horn on $v_{\mu}$ CC spectrum compared to the the MC before (blue) and after (red) both the hadron production and focusing fits. From Ref. [84]. 
Table 5.1: MINOS+ beam focusing systematics. Omitting the baffle scrapping systematic which was found to be negligible.

\begin{tabular}{lr}
\hline Source & Uncertainty \\
\hline Horn current miscalibration & $1 \mathrm{kA}$ \\
Material error & see text \\
Horn current distribution & $(\delta=\infty) /(\delta=0.77 \mathrm{~cm})$ \\
Horn 1 misalignment & $0.5 \mathrm{~mm}$ \\
Beam position & $0.5 \mathrm{~mm}$ \\
Proton beam width & $0.2 \mathrm{~mm}$ \\
Protons on target & $2 \%$ \\
\hline
\end{tabular}

downstream mounts by 1.37 mrad.

The bushing that failed is made of graphalloy, shown in Fig. 5.16. The horn components including their drive devices are exposed to high levels of radiation. As a result, they are susceptible to corrosion. This bushing was in the beamline since NuMI began operations in 2005 and very likely weakened by corrosion. Furthermore, the drive motors commonly must overcome the friction from corrosion of the horn shafts when positioning Horn 1.

Prior to the failure, Horn 1 was positioned at the start of 2015 operations. The hypothesis is the drive motor was fighting corrosion to move the horn, and the resulting friction destroyed the bushing. Without the bushing the drive rod moved up crushing the washer in Fig. 5.16 and creating backlash. At this point the only thing keeping Horn 1 in position was the friction from corrosion. The high current creates stress on the horns from Lorentz forces that is audible. The conclusion is that as the horn vibrated under stress 
the drive rod fell to the bottom of its housing causing the horn to drop. The graphalloy bushing were replaced with corrosion resistant brass to prevent this failure mode.

The horn fell during the last year of MINOS+ operations. The simulated flux at the ND for different fractions of the measured tilt is shown in Fig. 5.17. Below $8 \mathrm{GeV}$ there is a decrease in the number of $v_{\mu}$ events, and above $8 \mathrm{GeV}$ there is an increase in the number of $\boldsymbol{v}_{\mu}$ events as large as $80 \%$ between $10.5 \mathrm{GeV}$ and $11 \mathrm{GeV}$. The ratio of simulated flux for the measured horn tilt to the nominal flux is shown in Fig. 5.18.

The ND $v_{\mu}$-CC event rates in Fig. 5.20 stopped changing and stabilized during February 2016. The event rates remained stable until the end of running on June 29, 2016. On April 23, 2016 there was a fire that damaged a powersupply system for Fermilab's Recycler ring. With the Recycler offline the proton beam intensity was lower and the beam spot size decreased. This temporary change in running increased the number of events below $8 \mathrm{GeV}$ from April 24 to May 14, 2016. Figure 5.21 also shows the event rate at the muon monitor in alcove 3 no longer increasing as of February 2016. This information determined that the horn was likely in its final position by February $[97,98]$.

The 2016 year of data represents almost 50\% of the total exposure for MINOS+. In order to use this data, the ND was used to constrain the flux and a tilt systematic was added to the focusing systematic fit for the last MINOS+ run. The tilt systematic was chosen to be $1 / 4$ of the full $1.37 \mathrm{mrad}$ tilt. The fit was allowed to use the $3.425 \times 10^{-4} \mathrm{rad}$ tilt error band unconstrained. Two 


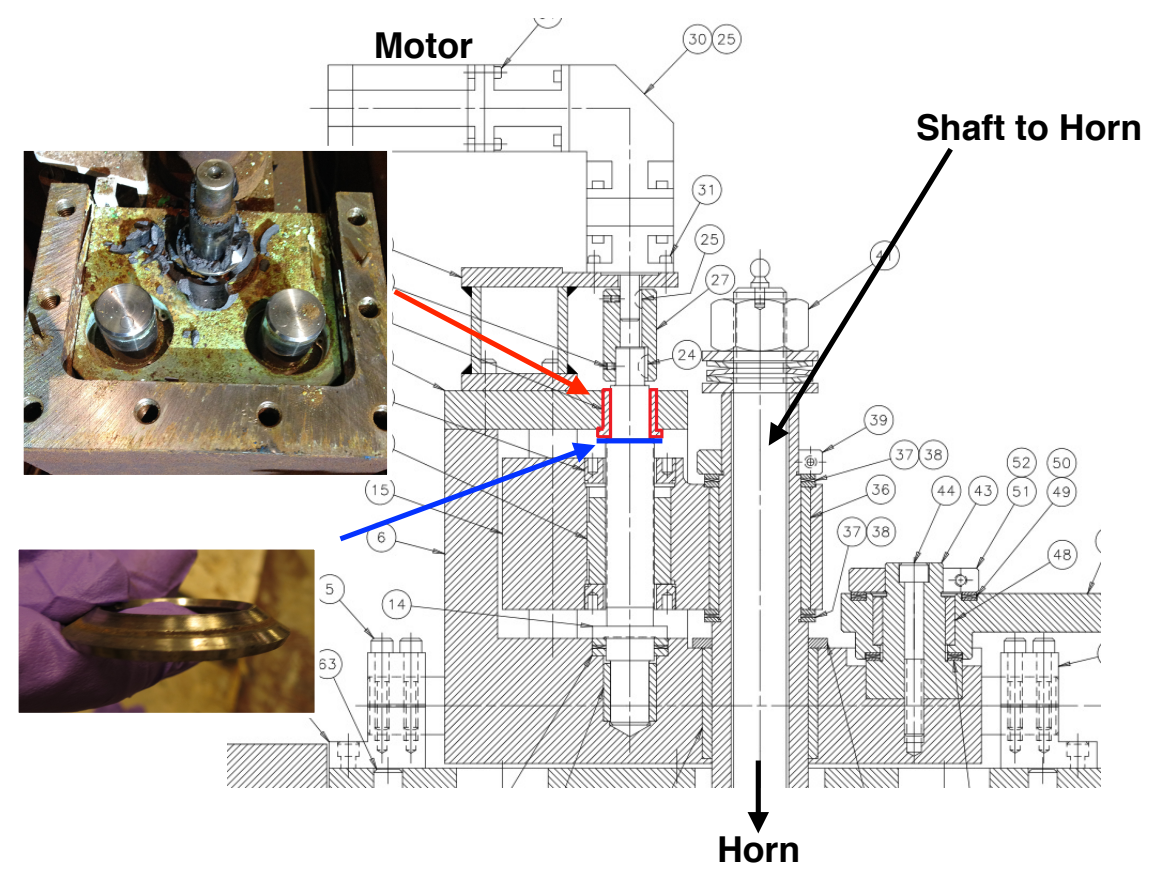

Figure 5.16: Schematic drawing of the bushing that failed highlighted in red which allowed horn 1 to fall and tilt. The washer highlighted in blue was bent by the horn positioning rod initially being pulled up passed the bushing housing after the bushing failed.

fits were done to the data with the horn tilt [99]. The first fit was done using ND data from February 16, 2016 to June 5, 2016. The second fit was done using the ND data from the entire 2015-2016 run.

When performing the fit to Run XIII, the horn current fit value was fixed from the fit to the value from the previous runs. The material systematic was fit in addition to the tilt since the tilted horn would change the amount of 


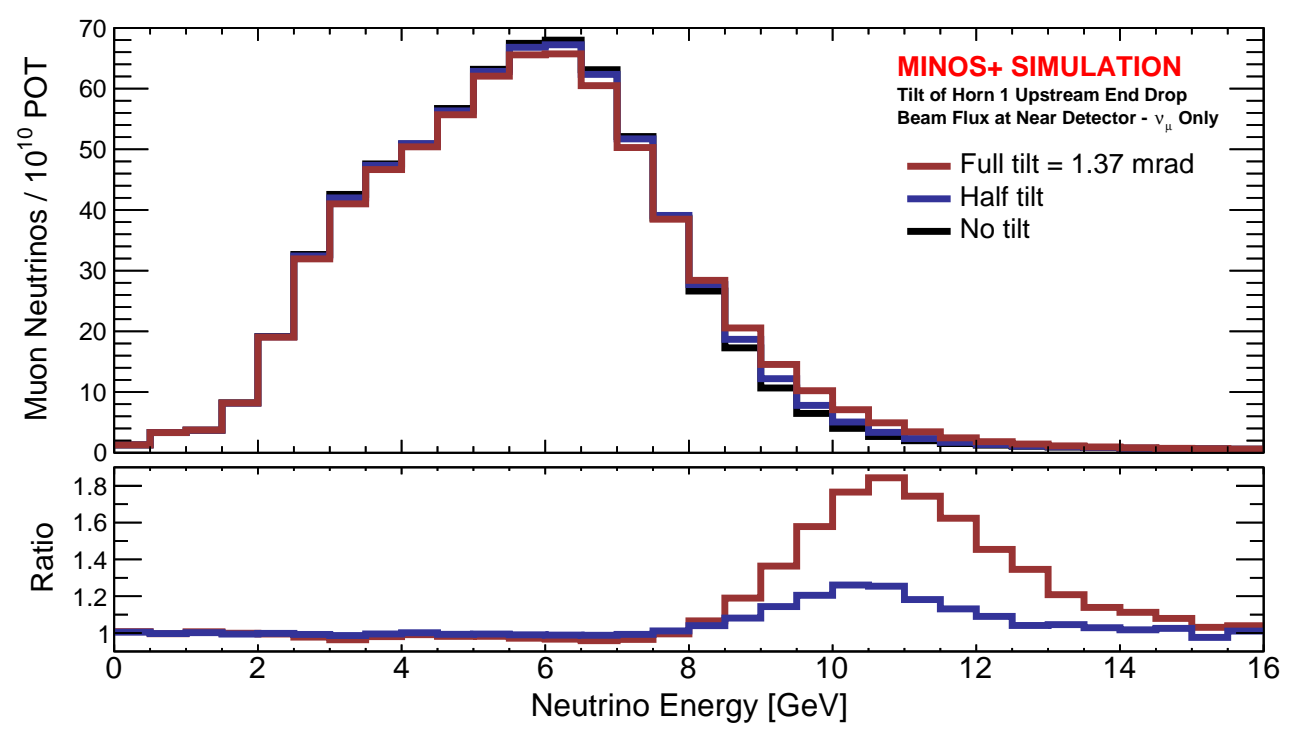

Figure 5.17: The simulated flux at the MINOS ND when the upstream end of horn 1 is lowered by different angles.

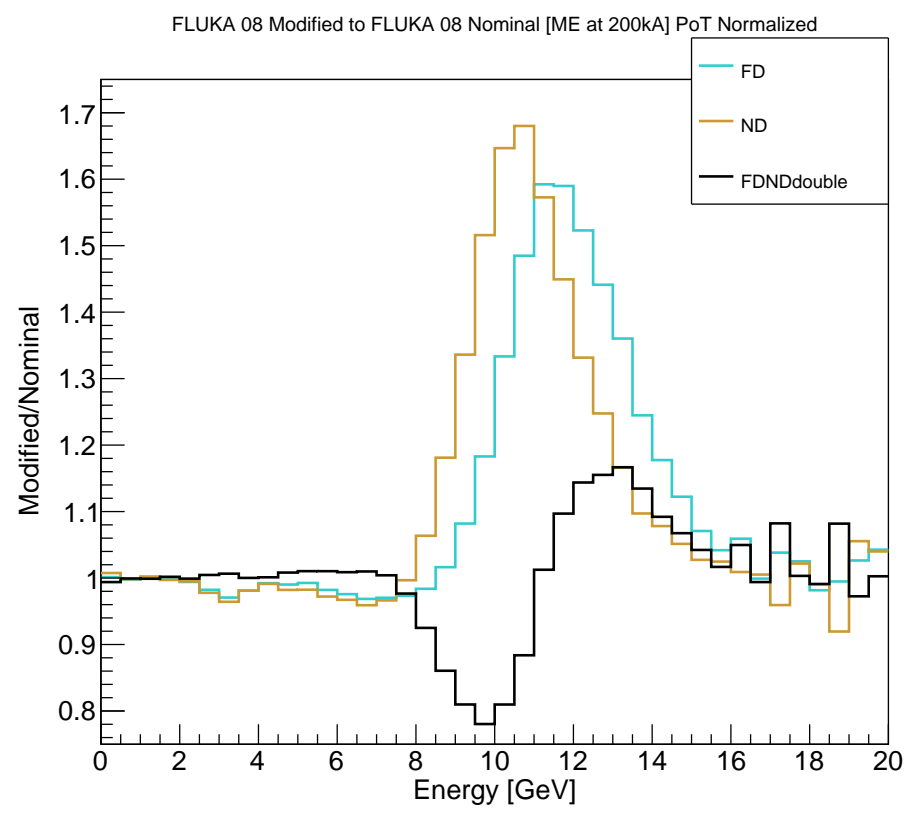

Figure 5.18: The ratio of the NuMI flux at the MINOS detectors for the upstream end of horn 1 lowered by $1.37 \mathrm{mrad}$ to the nominal flux. 


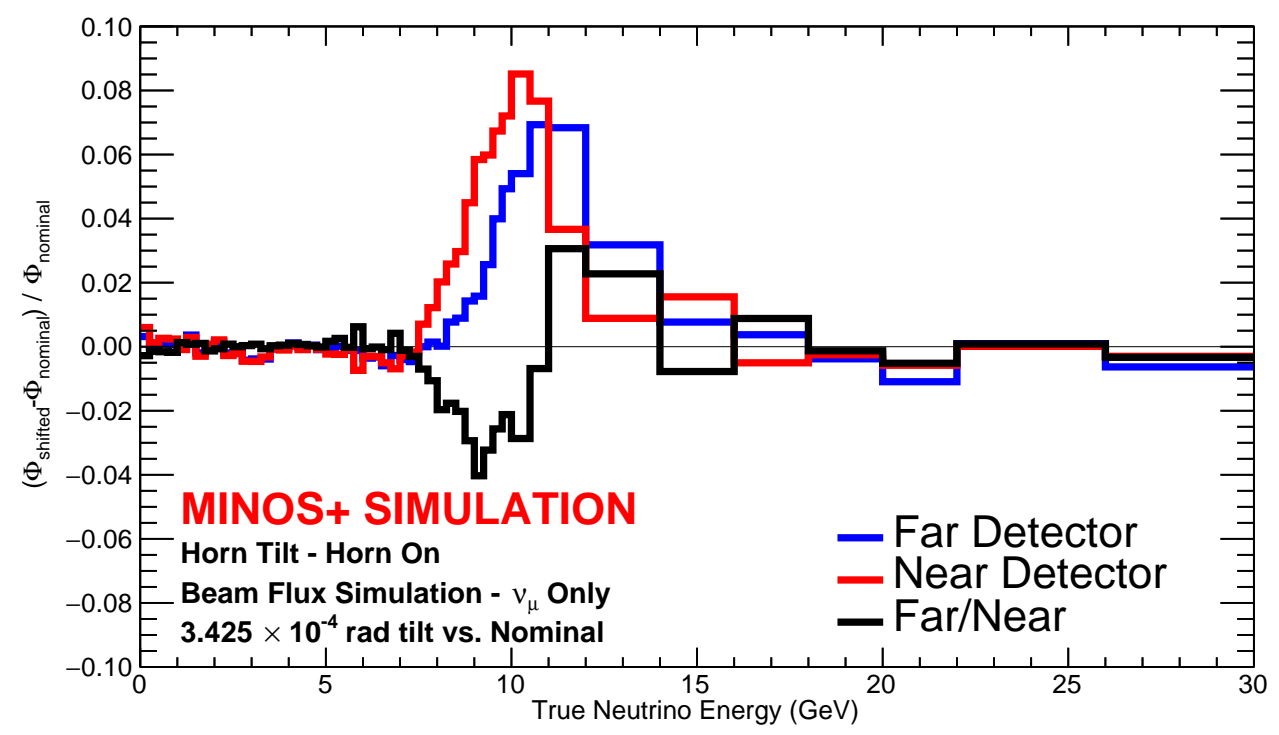

Figure 5.19: Horn tilt systematic band used to fit the Run 13 horn tilt.

material traversed by particles. In addition to fitting the whole run, a subset of the ND data was fit from February until the end of the run in June as a cross-check. The two fits produced consistent results. Their agreement implies that the horn fell soon after it was positioned, so the fit to the entire run period was used.

\subsection{Results}

The results of the beam fits to the MINOS+ runs are plotted in Figs. 5.9 and 5.22 to 5.24. Before fitting, there are differences on the data/MC ratio of up to 20\%. By constraining the simulated flux with ND data, the measured spectrum agrees with the tuned MC within the hadron production and focusing 

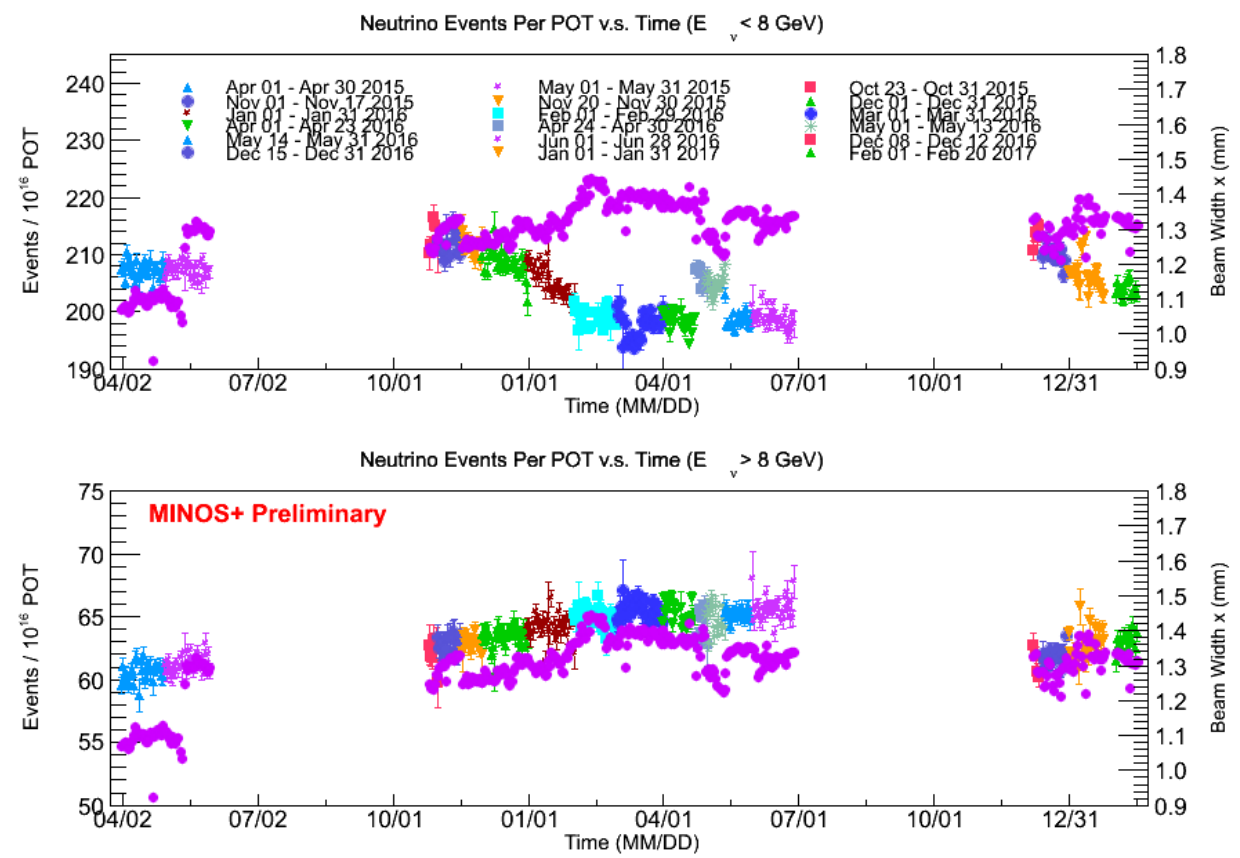

Figure 5.20: Near Detector $v_{\mu}$-CC event rate over time. The top plot shows the event rate for neutrino energy less than $8 \mathrm{GeV}$, and the bottom plot shows the event rate for neutrino energy greater than $8 \mathrm{GeV}$.

MM3

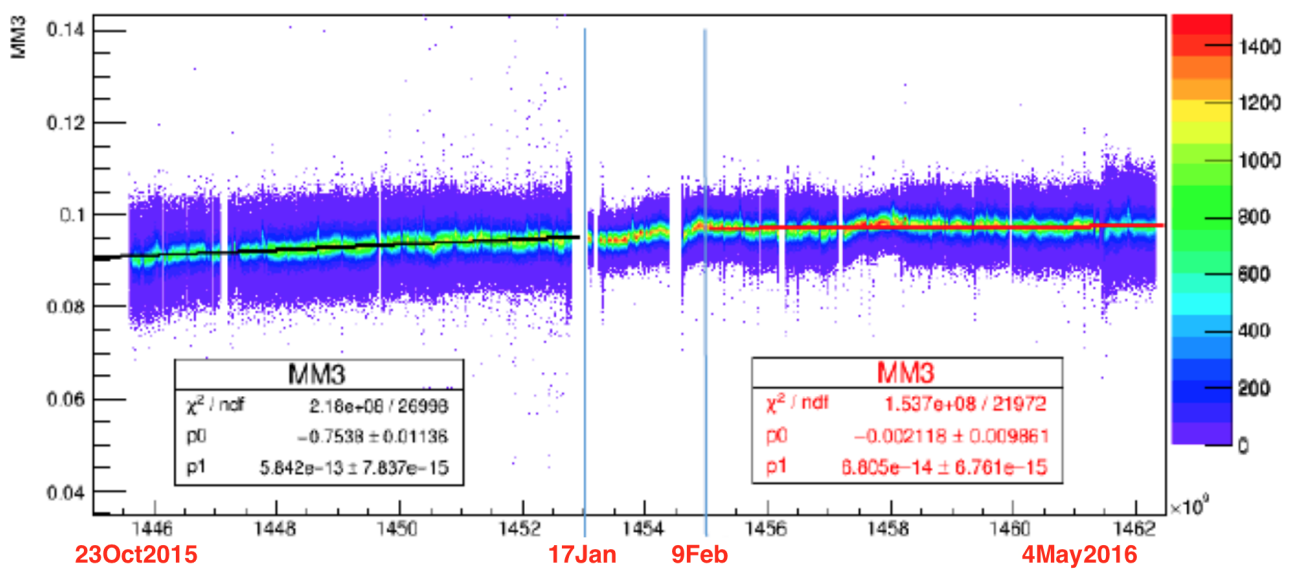

Figure 5.21: Event rate in arbitrary units at the NuMI muon monitor in alcove 3 from October 2015 to May 2016. The plot shows the event rate increasing within the period from October to February. This time period corresponds to when NuMI Horn 1 is believed to have tilted. From Ref. [97]. 
uncertainties. The tuned simulated flux can now be used to predict the flux at the FD.
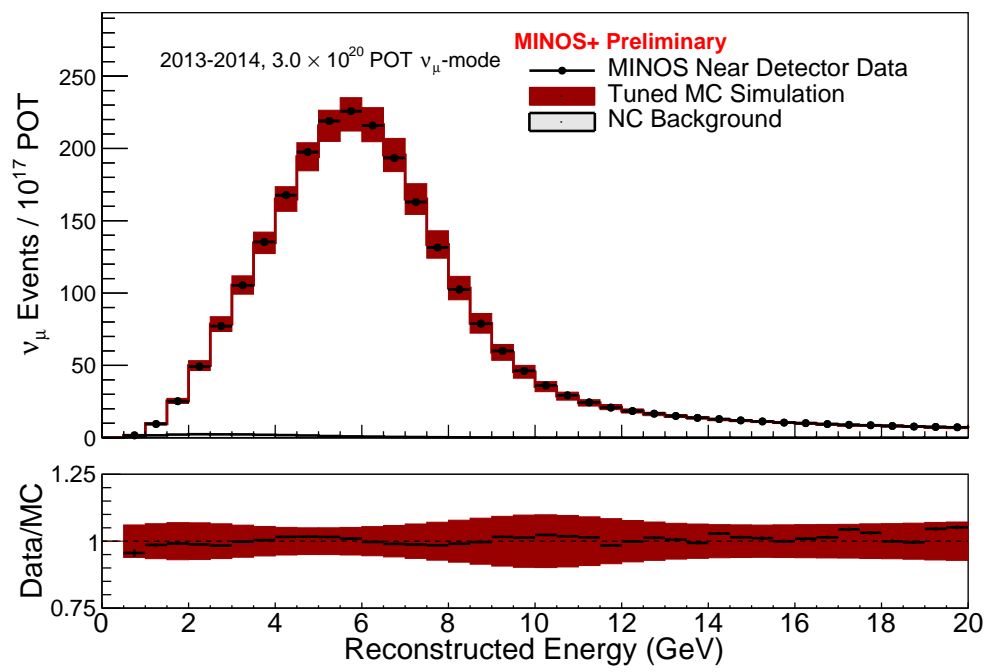

Figure 5.22: Reconstructed neutrino energy for MINOS+ Run 11 events in the near detector. The data spectrum in black is compared to the tuned simulated spectrum with $1 \sigma$ flux uncertainties in red. 

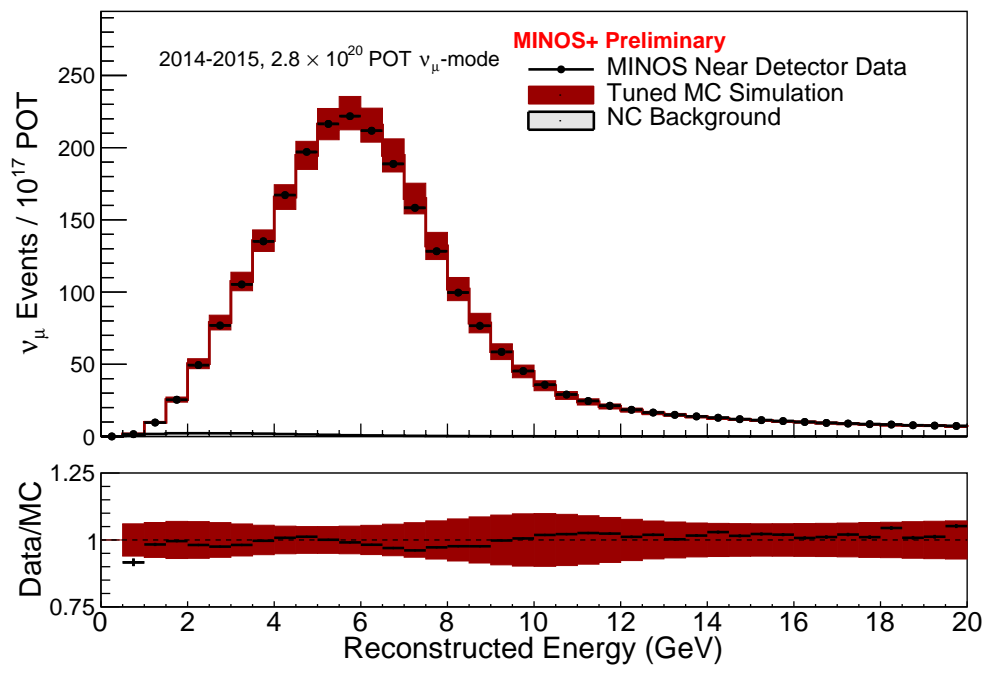

Figure 5.23: Reconstructed neutrino energy for MINOS+ Run 12 events in the near detector. The data spectrum in black is compared to the tuned simulated spectrum with $1 \sigma$ flux uncertainties in red.
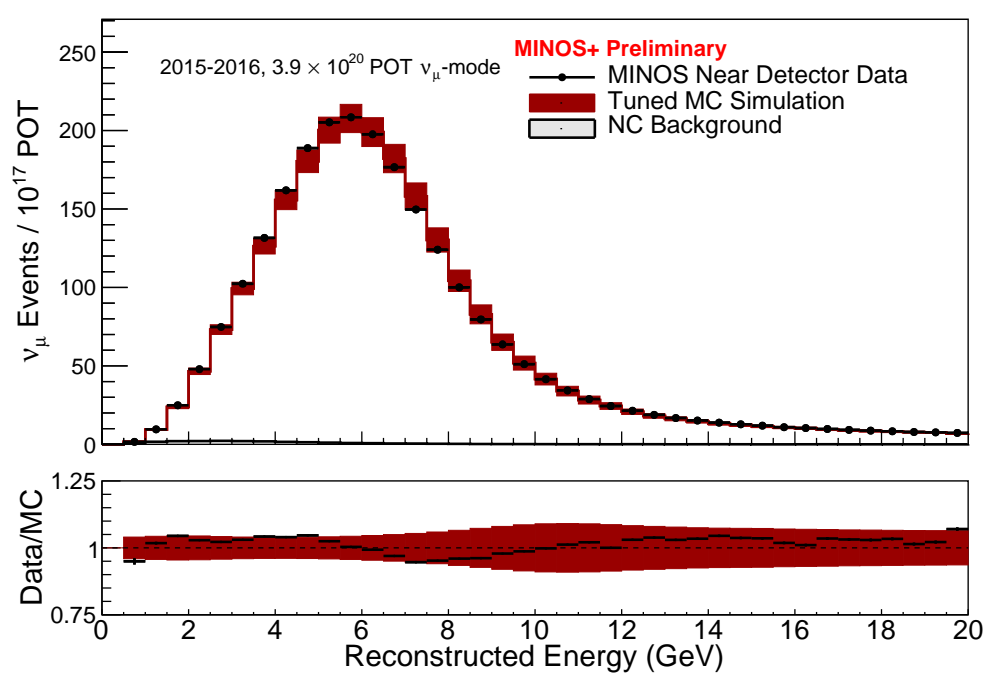

Figure 5.24: Reconstructed neutrino energy for MINOS+ Run 13 events in the near detector. The data spectrum in black is compared to the tuned simulated spectrum with $1 \sigma$ flux uncertainties in red. 


\section{Chapter 6}

\section{The Three-Flavor Analysis}

The MINOS+ three-flavor analysis uses neutrino data from the NuMI

beam and the atmosphere to measure $\Delta m_{32}^{2}$ and $\sin ^{2} \theta_{23}$. This chapter summarizes how the different data sets are incorporated into the three-flavor analysis with an emphasis on the features relevant for the MINOS+ beam data.

In general terms, the analysis compares data spectra to predicted spectra that have been weighted by three-flavor oscillation probabilities in matter. Different methods are employed for generating predicted spectra for the beam and atmospheric neutrino samples.

\subsection{Beam Analysis Strategy}

The beam analysis compares the measured FD spectrum to the predicted FD spectrum. MINOS+ uses the same method developed for MINOS whereby FD predictions are generated by extrapolating the measured ND spectrum to the FD. The MINOS and MINOS+ beam data sets are comprised of multiple run periods, and each defined beam run period is separately extrapolated.

The extrapolation method used by MINOS and MINOS+ for this anal- 
ysis is referred to as the beam matrix method. The extrapolation starts from the sample of muon (anti)neutrinos selected at the ND. The ND spectrum is then unfolded into a ND flux in true neutrino energy by applying the selection purity and efficiency determined from MC. The ND flux is then extrapolated to the FD by multiplying by a transfer matrix known as the beam matrix. This multiplication produces an unoscillated FD flux. At this point, oscillation probabilities can be applied to the FD flux. The predicted FD spectrum is achieved by applying the event selection purity and efficiency for the FD.

\subsection{Unfolding}

The unfolding of the ND spectrum begins by applying a purity correction in reconstructed neutrino energy to derive the true CC spectrum. Applying the purity correction removes $\mathrm{NC}$ events and $\bar{v}_{\mu}\left(\boldsymbol{v}_{\mu}\right)$ events that contaminate the selected $v_{\mu}\left(\bar{v}_{\mu}\right)$ sample. Purity has the same definition for the $\mathrm{ND}$ and FD. The purity quantifies the fraction of simulated CC events in the $\mathrm{MC}$ sample that pass the CC selection criteria. The purity for each detector is calculated from MC for each reconstructed energy bin. The purity is defined by the ratio in Eq. 6.1.

$$
P_{i}=\frac{N_{i}\left(\text { selected true CC } v_{\mu}\right)}{N_{i}\left(\text { all selected CC } v_{\mu}\right)},
$$

where $P$ is the purity, $i$ designates the bin of reconstructed energy, and $N$ is the number of events meeting the criteria in parentheses. Figure 6.1 shows the purity as a function of reconstructed neutrino energy for a typical MINOS+ 


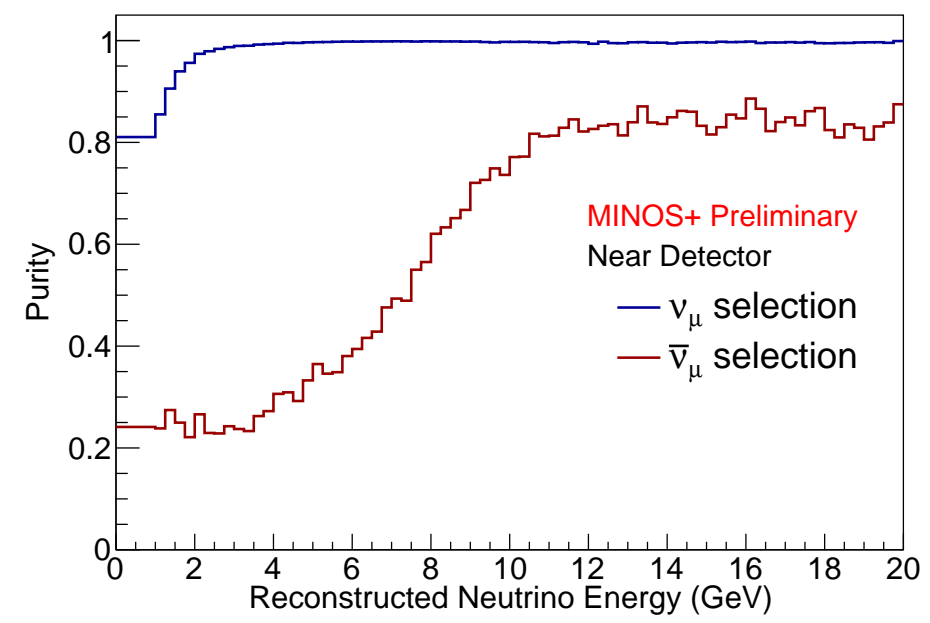

Figure 6.1: MINOS+ Run 12 ND purity for selected $v_{\mu}$ and $\bar{v}_{\mu}$ events as a function of reconstructed neutrino energy.

Table 6.1: MINOS+ Run XII total purities for selecting $v_{\mu}$ or $\bar{v}_{\mu}$ CC events.

\begin{tabular}{cc}
\hline Detector & Purity (\%) \\
\hline Near & 99.1 \\
Far & 99.3 \\
\hline
\end{tabular}

run period. The total purity is then

$$
P=\frac{\sum_{i=1}^{\text {Nbins }} N_{i}\left(\text { selected true CC } v_{\mu}\right)}{\sum_{j=1}^{N \text { Nbins }} N_{j}\left(\text { all selected CC } v_{\mu}\right)} .
$$

Total purities are calculated for a representative MINOS+ run in Table 6.1.

With expected NC and wrong-charge events subtracted from the ND sample, the spectrum is then converted to true neutrino energy via a matrix mapping reconstructed to true energy made from the MC. The ND Reconstructed- 
to-True Energy matrix is filled for each reconstructed true CC event. The matrix is then normalized using the reconstructed energy spectrum for the true CC events. This normalized matrix produces the true energy spectrum per reconstructed neutrino. A separate matrix is made for the $v_{\mu}-\mathrm{CC}$ and $\bar{v}_{\mu}$-CC samples, as shown in Fig. 6.2.
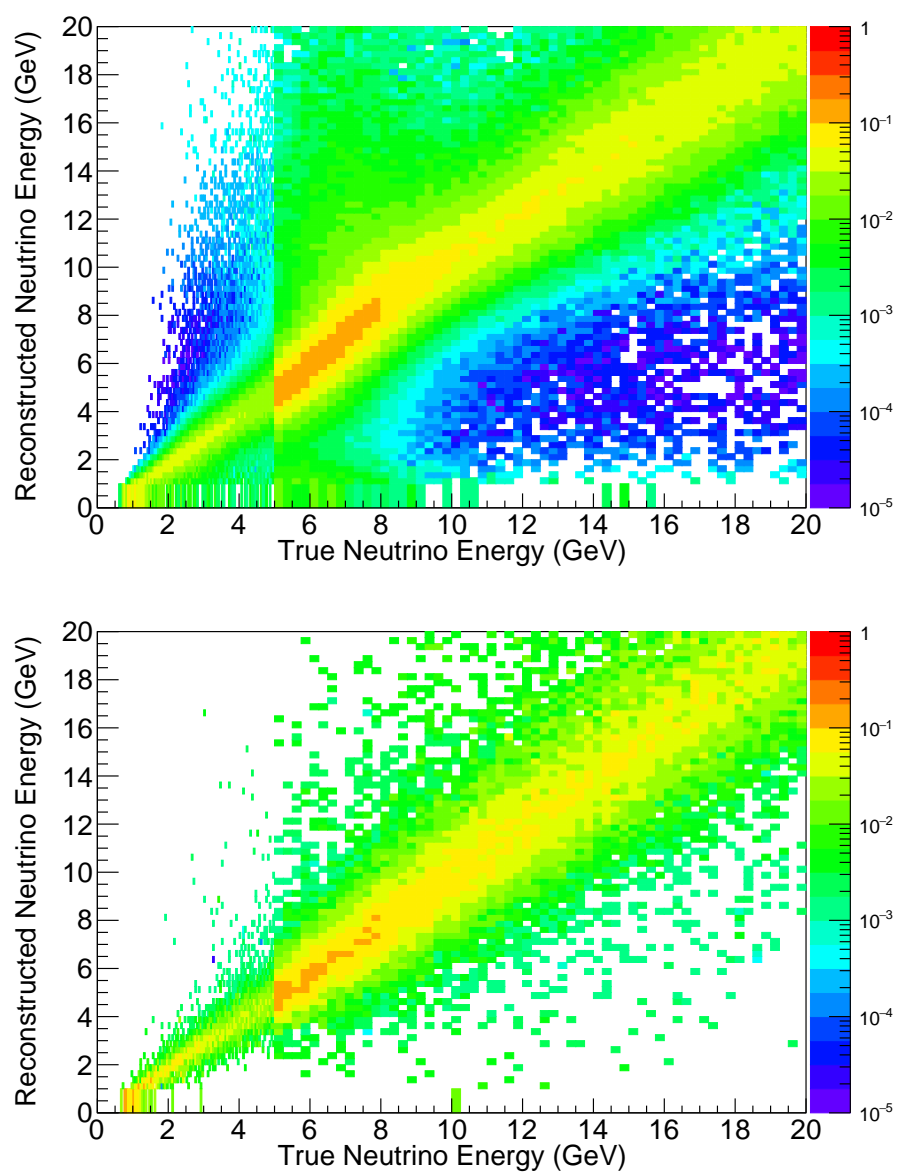

Figure 6.2: MINOS+ Run 12 ND Reconstructed Neutrino Energy vs True Neutrino Energy for selected $v_{\mu}$ (top) and $\bar{v}_{\mu}$ (bottom) events used to unfold the ND spectrum in true neutrino energy. 
The Reconstructed-to-True Energy matrix in Fig. 6.2 exhibits features of the analysis binning and the neutrino beam. The true neutrino energy is divide into fine bins of $0.05 \mathrm{GeV}$ in the region of the expected oscillation maximum from 0 to $5 \mathrm{GeV}$. At $5 \mathrm{GeV}$ the binning size increases to $0.25 \mathrm{GeV}$ resulting in the feature at $5 \mathrm{GeV}$. Since the neutrino beam is focused around $7 \mathrm{GeV}$, MINOS+ measures few events at low energies. To account for the low number of events, the reconstructed neutrino energy uses a single bin from 0 to $1 \mathrm{GeV}$. Additionally, the $\boldsymbol{v}_{\mu}$ matrix peaks between 5 and $8 \mathrm{GeV}$ reflecting the neutrino-mode beam focusing.

Once the ND spectrum is transformed into true energy, an efficiency correction is applied to generate the expected spectrum from CC interactions in the ND fiducial volume. The efficiency is defined the same way for both detectors, and it quantifies the fraction of simulated $\mathrm{CC}$ interactions in the detector fiducial volume that satisfy the selection criteria. The efficiency for each detector is calculated from MC for each true neutrino energy bin without applying oscillation probabilities. The efficiency is defined by the ratio

$$
E_{i}=\frac{N_{i}\left(\text { selected true CC } v_{\mu} \text { events }\right)}{N_{i}\left(\text { true } v_{\mu} \text { CC events in the fiducial volume }\right)},
$$

where $E$ is the efficiency, $i$ designates the bin of true neutrino energy, and $N$ is the number of events meeting the criteria in parentheses. In this ratio, the numerator is binned according to the true visible neutrino energy in the detector, and the denominator is binned according to the true neutrino energy. True visible neutrino energy is defined as total event energy deposited in the 
detector. This definition affects events with showers since the track energy is determined from range or curvature. The calculated efficiency for the ND is shown in Fig. 6.3 as a function of true neutrino energy. The total efficiency is

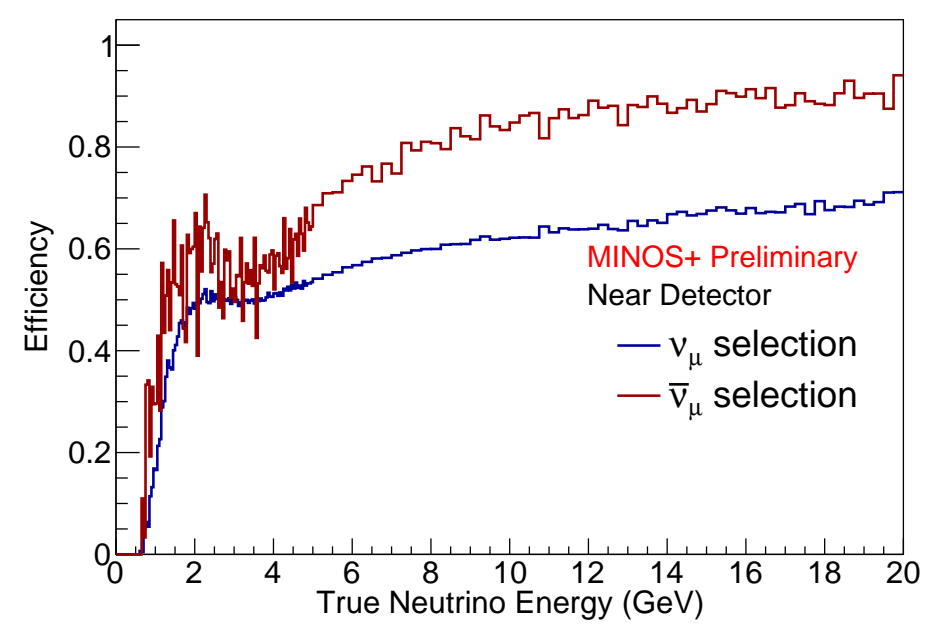

Figure 6.3: MINOS+ Run 12 ND efficiency for selected $\nu_{\mu}$ and $\bar{v}_{\mu}$ events as a function of true neutrino energy.

thus

$$
E=\frac{\sum_{i=1}^{N b i n s} N_{i}\left(\text { selected true CC } \gamma_{\mu} \text { events }\right)}{\sum_{j=1}^{N b i n s} N_{j}\left(\text { true } v_{\mu} \text { CC events in the fiducial volume }\right)} .
$$

Total efficiencies are calculated for a representative MINOS+ run in Table 6.2. The true energy spectrum of $\mathrm{CC}$ events in the fiducial volume is then divided by the fiducial mass of the ND and the CC cross section to yield the neutrino flux at the ND. The flux at the ND is then used to predict the flux at the FD. 
Table 6.2: MINOS+ Run XII total efficiencies for selecting $v_{\mu}$ or $\bar{v}_{\mu}$ CC events.

\begin{tabular}{cc}
\hline Detector & Efficiency (\%) \\
\hline Near & 58.7 \\
Far & 84.1 \\
\hline
\end{tabular}

\subsection{Extrapolation: Beam Matrix}

If the neutrino beam was created from a point source, the flux at the FD would simply be the ND flux scaled by the square of the ratio of the sourcedetector distances. However, the actual neutrino source is the distribution of focused mesons from the graphite target to the end of the decay pipe. The distributed source of neutrinos introduces differences between the flux measured at the ND and at the FD. These differences manifest as a result of the angular acceptance of each detector with respect to meson decays occurring along the beamline. The illustration in Fig. 6.4 shows that since the ND is closer to the decay pipe there is a larger range of possible decays that will result in a neutrino passing through the ND compared to the FD, especially for decays occurring at the downstream end of the decay pipe. Figure 6.5 shows that mesons with identical momenta produce different energy spectra at each detector. This is illustrated with the shaded regions, where the ND ranges correspond to smeared and skewed ranges at the FD. The neutrino energy in the lab frame can be expressed as

$$
E_{v}=\frac{E_{v}^{*}}{\gamma_{\mathrm{p}}\left(1-\beta_{\mathrm{p}} \cos \theta\right)}
$$




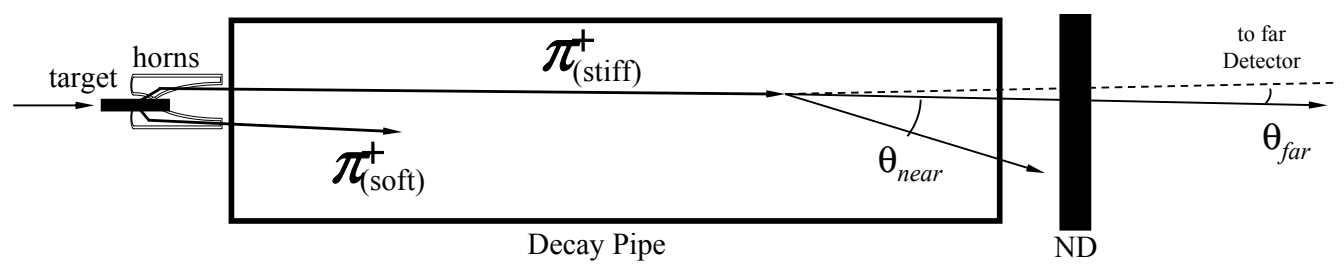

Figure 6.4: Illustration of the angular acceptance of the Near Detector and Far Detector. From Ref. [40].
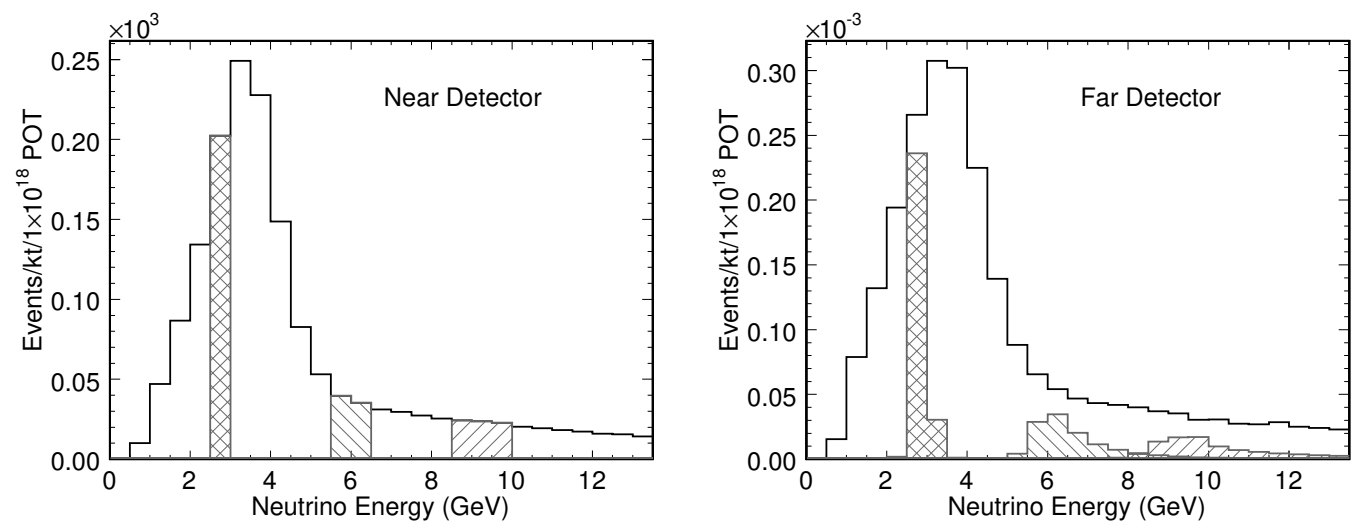

Figure 6.5: Illustration of the effect of the angular acceptance of the Near Detector and Far Detector on the observed flux. From Ref. [40]. 
where $E_{v}^{*}$ is the energy of the neutrino in the parent's rest frame, $\gamma_{\mathrm{p}}$ is the parent's Lorentz factor, $\beta_{\mathrm{p}}$ is the parent's velocity, and $\theta$ is the angle between the momentum of the parent meson and momentum of the neutrino in the lab frame. Equation 6.5 shows that the neutrino energy is inversely proportional to the angle $\theta$. This relationship explains the skewing of higher neutrino energies observed in the FD spectrum in Fig. 6.5. Since the FD is a significant distance from the source, the FD samples a smaller range of decay angles than the ND.

Although the neutrino flux is different between the ND and FD, both detectors are sampling the neutrinos produced by the same distribution of meson decays. This suggests that the flux of the two detectors is correlated. In order to find the correlation, it is necessary to understand the angular distribution of neutrinos from the meson decay.

In the parent's frame, the decay resulting in a neutrino and charged lepton is isotropic. After transforming to the lab frame, the angular distribution of the daughter neutrino has the form:

$$
\frac{d N}{d \cos \theta}=\frac{1}{2 \gamma_{\mathrm{p}}^{2}\left(1-\beta_{\mathrm{p}} \cos \theta\right)^{2}} .
$$

Then the probability of the neutrino passing through an area, $A$, is

$$
P=\int_{A} \frac{1}{2 \pi} \frac{d N}{d \cos \theta} d \Omega .
$$

At the MINOS detectors, $\Omega_{A}$ is the solid angle subtended by $A$, a distance, $d$, from the decay point. Since $d$ is sufficiently large, the solid angle 
is small, and the angular distribution of the neutrino can be approximated as constant over A. Thus, Eq. 6.7 becomes

$$
\begin{aligned}
P & \left.\approx \frac{1}{2 \pi} \frac{d N}{d \cos \theta}\right|_{\theta_{D}} \Omega_{A} \\
& \left.\approx \frac{1}{2 \pi} \frac{d N}{d \cos \theta}\right|_{\theta_{D}} \frac{A}{d^{2}} .
\end{aligned}
$$

Using Eqs. 6.5 and 6.8, the partial neutrino flux as a function of neutrino energy at each detector can be calculated for a meson. For a given meson, there is a range of possible neutrino energies for neutrinos that could pass through the ND. For that same meson, there is a partial neutrino flux at the FD. This partial neutrino flux is correlated with the possible neutrino energies at the ND. Consequently, for a single meson there is a two-dimensional NDto-FD neutrino energy matrix with each element equal to the FD partial flux at the corresponding neutrino energy. The sum of all the meson matrices correlates the total ND flux for the given meson distribution to the total FD flux. The total FD flux for a particular FD neutrino energy is equal to the sum over all the ND neutrino energy elements. Finally, the matrix is normalized by the total ND flux so that the matrix produces the appropriate FD flux when multiplied by a ND flux. This normalized matrix is called a beam matrix. This technique was originally proposed by M. Szleper and A. Para [100] and treated in detail in Refs. [57,101].

In practice, the beam matrix is constructed using Monte Carlo integration. The distribution of parent mesons comes from the beamline simulation. For each meson, ten random points are chosen in the ND fiducial volume. The 
energy and probability are calculated for each of the ten random neutrino rays passing through a small area at the detector. For the calculation, the area is a circle with $r=1 \mathrm{~m}$. This is a sensible area that is on the same scale as the detector face, and it is sufficiently small that at long distances the flux can be assume constant within the circle. For each decay pointing to the ND, the energy and probability is calculated for a neutrino ray passing through the same size circle at the face of the FD. The probability for each FD neutrino ray is added to the beam matrix at the element of its FD neutrino energy and the associated random ND neutrino energy. As above, the matrix is normalized with total ND flux.

The FD flux is then calculated by multiplying the derived ND flux by the the beam matrix shown in Fig. 6.6.

\subsection{Folding}

Once the FD flux is obtained from the beam matrix, the flux is made into a predicted FD spectrum by applying the reverse of the unfolding that was done to the ND spectrum. The FD flux is multiplied by the cross section and fiducial mass to get the number of predicted $\mathrm{CC}$ interactions in the detector. This predicted spectrum is then multiplied by the efficiency to get the number of $\mathrm{CC}$ interactions that are selected. Equation 6.3 is used to calculate the efficiency shown in Fig. 6.7 as a function of true neutrino energy. Table 6.2 compares the total efficiency of the ND and FD.

At this point, the predicted true neutrino energy spectrum of selected 

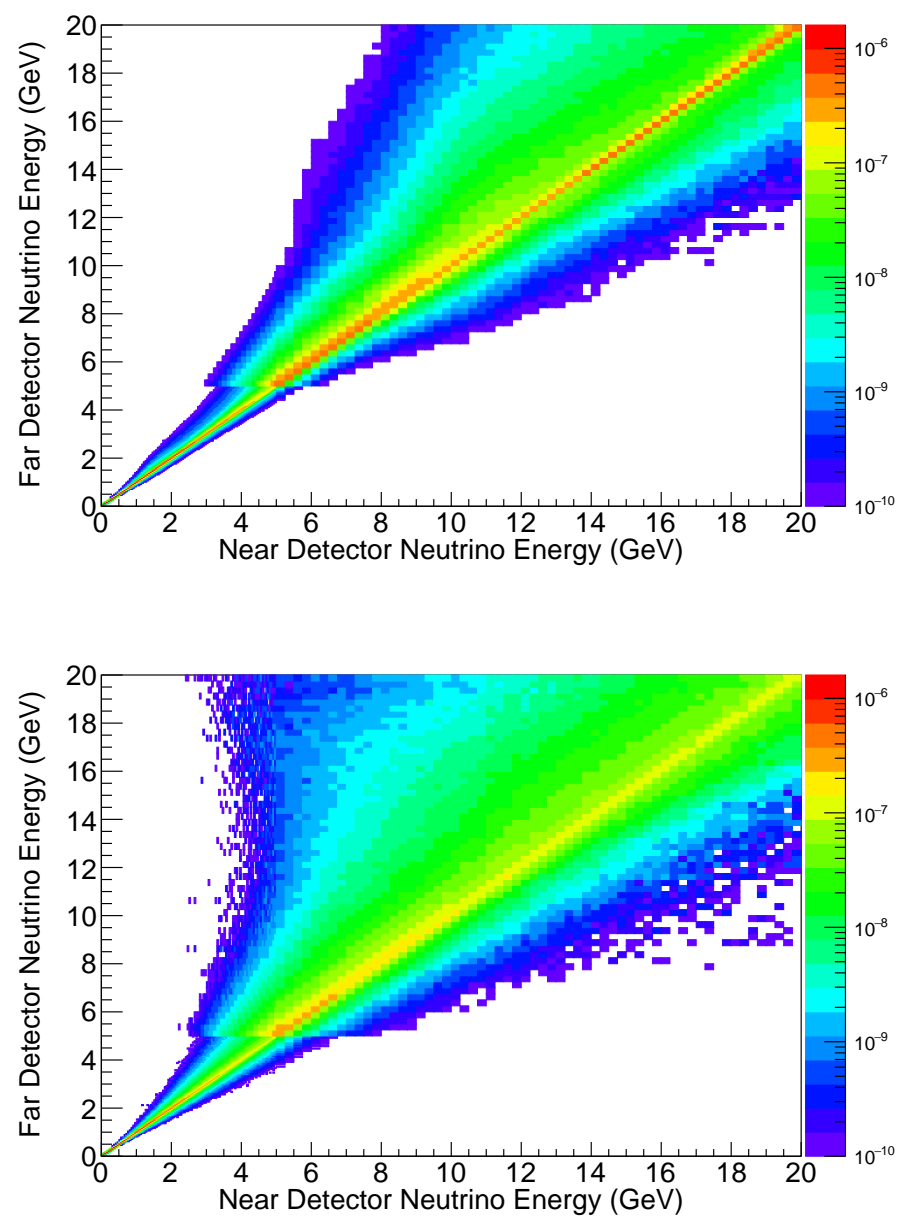

Figure 6.6: The beam matrix used for MINOS+ Run $12 v_{\mu}$ (top) and $\bar{v}_{\mu}$ (bottom) events. 


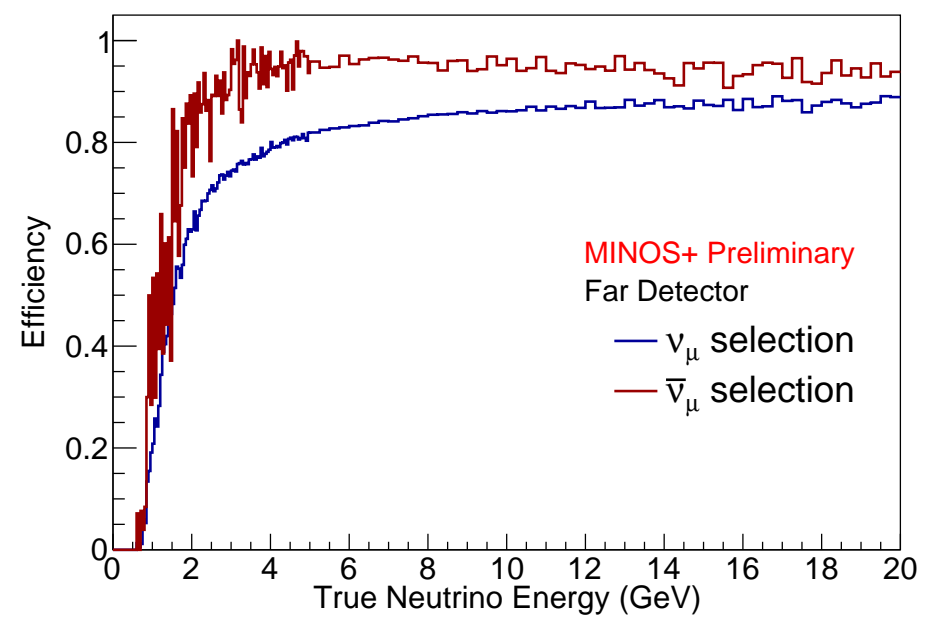

Figure 6.7: MINOS+ Run 12 FD efficiency for selected $v_{\mu}$ and $\bar{v}_{\mu}$ events as function of true neutrino energy.

events can be oscillated. The exact three-flavor oscillation probabilities in matter are applied. The analysis uses the hybrid ZHEEVH3 algorithm described in Ref. [102] to diagonalize the Hamiltonian matrix,

$$
H=\frac{1}{2 E} U\left(\begin{array}{ccc}
0 & 0 & 0 \\
0 & \Delta m_{21}^{2} & 0 \\
0 & 0 & \Delta m_{31}^{2}
\end{array}\right) U^{\dagger}+\left(\begin{array}{ccc} 
\pm \sqrt{2} G_{\mathrm{F}} n_{\mathrm{e}} & 0 & 0 \\
0 & 0 & 0 \\
0 & 0 & 0
\end{array}\right)
$$

In Eq. 6.9, $U$ is the PMNS mixing matrix from Eq. 1.5, $G_{\mathrm{F}}$ is the Fermi Constant, and $\sqrt{2} G_{\mathrm{F}} n_{\mathrm{e}}$ is the $\mathrm{CC}$ potential for electron neutrinos. The potential is positive for neutrinos and negative for antineutrinos. The average electron density of the neutrino beam's path was calculated as $n_{\mathrm{e}}=1.36 \frac{\mathrm{mol}}{\mathrm{cm}^{3}}$ [103]. With the Hamiltonian diagonalized, the neutrino flavor state is propagated, using the eigenvalues and eigenvector matrix.

The resultant spectrum is converted to reconstructed energy using a 
Reconstructed-to-True Energy matrix. Figure 6.8 shows the FD Reconstructedto-True Energy matrix, which is normalized by the true CC event spectrum such that matrix multiplication produces the reconstructed energy spectrum per true CC interaction.
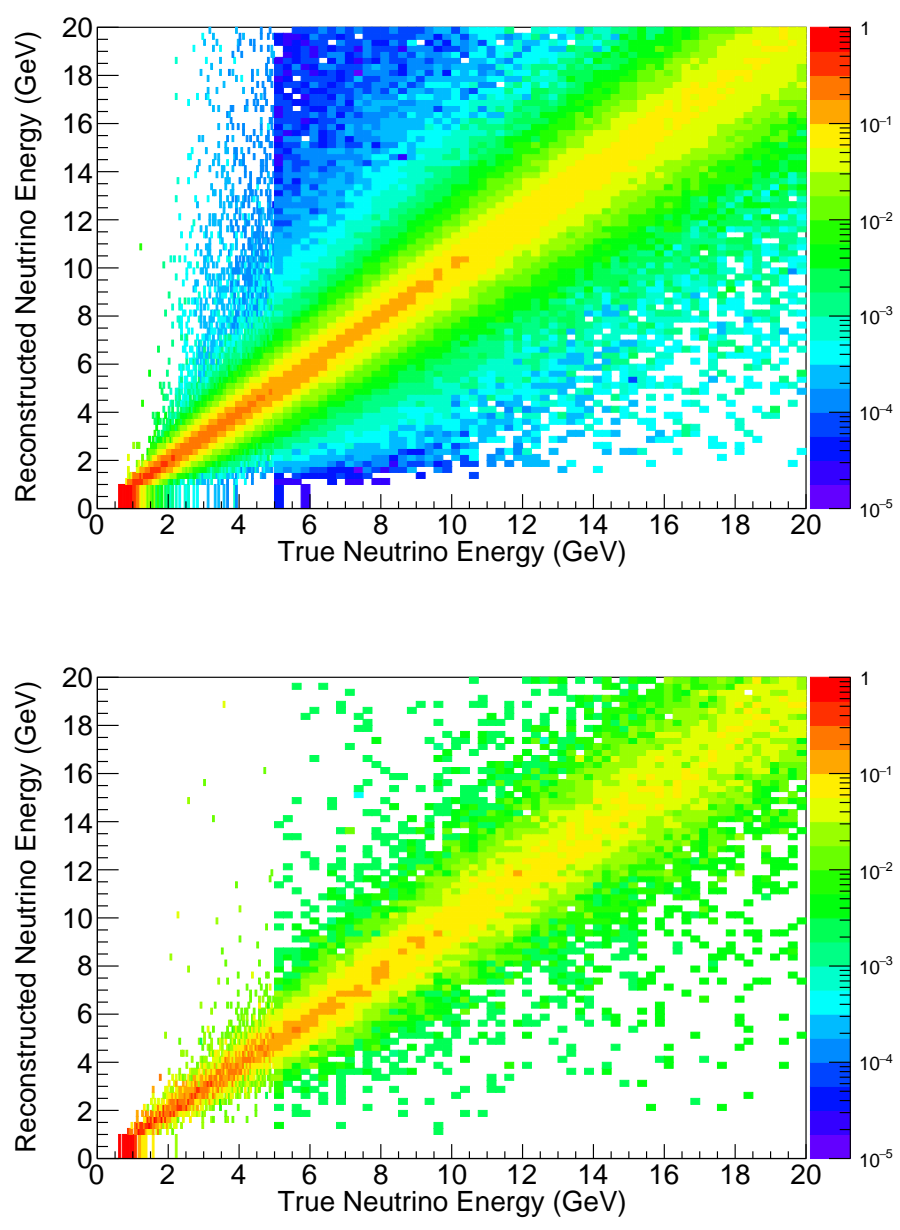

Figure 6.8: MINOS+ Run 12 FD Reconstructed Neutrino Energy vs True Neutrino Energy for selected $v_{\mu}$ (top) and $\bar{v}_{\mu}$ (bottom) events. 
The reconstructed energy spectrum is then multiplied by the selection purity to create a prediction of the measured spectrum. The FD purity shown in Fig. 6.9 is calculated using Eq. 6.1. Table 6.1 compares the total purity of the ND and FD samples.

The final predicted spectrum at the FD includes backgrounds from the corresponding antineutrinos, $\mathrm{NC}$ interactions, and tau neutrinos in the case of oscillations.

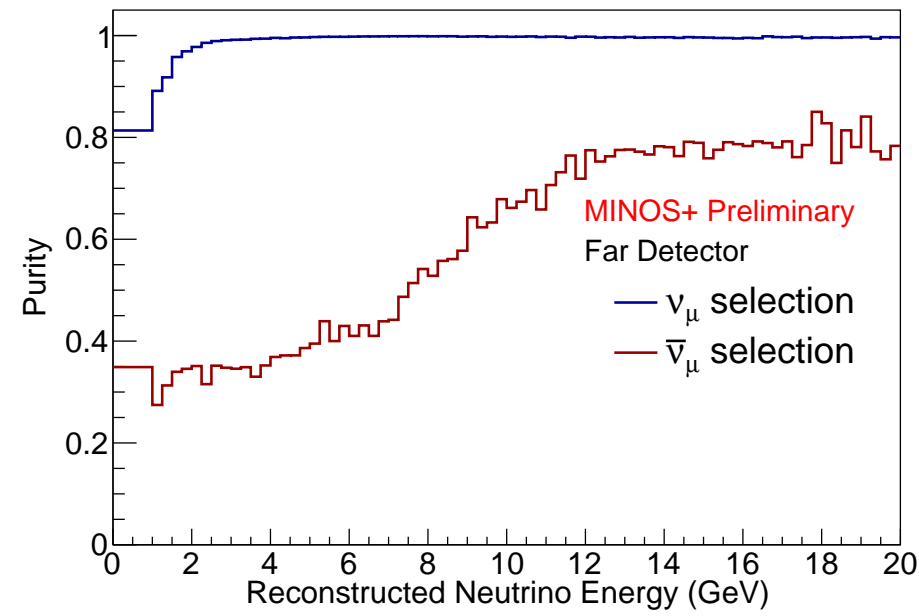

Figure 6.9: MINOS+ Run $12 \mathrm{FD}$ purity for selected $\boldsymbol{v}_{\mu}$ and $\overline{\boldsymbol{v}}_{\mu}$ events as a function of reconstructed neutrino energy.

\subsection{Atmospheric neutrinos}

The full $\boldsymbol{v}_{\mu}$-disappearance sample includes 13 years of atmospheric neutrino exposure. The current analysis uses the same techniques described in 
Table 6.3: Earth density profile used to account for matter effects in the atmospheric neutrino oscillation analysis.

\begin{tabular}{lrr}
\hline Region & Radius $[\mathrm{km}]$ & $n_{\mathrm{e}}\left[\frac{\mathrm{mol}}{\mathrm{cm}^{3}}\right]$ \\
\hline Crust & $r \geq 6336$ & 1.45 \\
Mantle & $3470 \leq r<6336$ & 2.25 \\
Outer core & $1220 \leq r<3470$ & 5.15 \\
Inner core & $r<1220$ & 6.05 \\
\hline
\end{tabular}

Ref. [68]. The flux of atmospheric neutrinos at the FD is predicted using the model from Barr et al. [104] as a function of the cosine of the zenith angle and the neutrino energy. Three-flavor oscillations are applied to the predicted spectra of atmospheric neutrinos by solving the Hamiltonian in Eq. 6.9. However, the atmospheric neutrino propagation uses a four-layer model for the Earth to better estimate the contribution of matter effects to oscillation probabilities. Each layer's density is derived from the Preliminary Reference Earth Model (PREM) [105]. The four layers are defined in Table 6.3.

\subsection{The Fit to the Oscillation Parameters}

The predicted FD spectrum can then be used to fit to the FD data for oscillation parameters. For this analysis, the selected beam and atmospheric muon (anti)neutrino spectra are fit using a set of predictions for different oscillation parameters called templates. Templates are folded predictions produced by the beam matrix method. The oscillation parameters used in the templates form a multi-dimensional grid in $\left(\sin ^{2} \theta_{23}, \Delta m_{32}^{2}, \sin ^{2} \theta_{13}, \delta_{\mathrm{CP}}\right)$. Predictions for 
arbitrary oscillation parameters are achieved through interpolation of the template predictions.

\subsubsection{Fit Statistic}

Minuit [106] is used to minimize the statistic,

$$
\chi^{2}=-2 \ln \lambda+\text { Penalty Terms, }
$$

where $\lambda$ is the likelihood ratio for Poisson distributed data. The likelihood ratio takes the following form $[26,107]$ :

$$
\lambda=\prod_{i} \exp \left(-\nu_{i}+n_{i}\right)\left(\frac{\nu_{i}}{n_{i}}\right)^{n_{i}} .
$$

In Eq. 6.11, $\nu_{i}$ is the predicted number of events in reconstructed energy bin $i$ and $n_{i}$ is the observed number of events in reconstructed energy bin $i$.

The fit has penalty terms that account for the uncertainty on the oscillation parameter $\theta_{13}$ as well the systematic uncertainties. These uncertainties are treated as nuisance parameters in the fit. The penalty terms have the form:

$$
\text { Penalty Terms }=\sum_{j}\left(\frac{\Delta \alpha_{j}}{\sigma_{\alpha_{j}}}\right)^{2},
$$

where $\Delta \alpha_{j}$ is the difference from the central value of nuisance parameter $\alpha_{j}$ and $\sigma_{\alpha_{j}}$ is the uncertainty of the nuisance parameter $\alpha_{j}$. The fit keeps the solar oscillation parameters fixed to $\Delta m_{21}^{2}=7.54 \times 10^{-3} \mathrm{eV}^{2}$ and $\sin ^{2} \theta_{12}=0.307$ [108]. The $\theta_{13}$ mixing angle is constrained using $\sin ^{2} \theta_{13}=0.0210 \pm 0.0011$ [109]. 


\subsubsection{Independent Constraints}

Chi-square $\left(\chi^{2}\right)$ values from independent experiments can be added to the fit statistic in Eq. 6.10 to provide additional constraints to the fit of muon neutrino disappearance. For the full three-flavor fit using all available MINOS and MINOS+ data, the $v_{\mathrm{e}}$-appearance analysis [58] is treated as independent, and the $\chi^{2}$ values from a four-dimensional parameter space are added as a constraint.

\subsection{Systematics}

The systematics used for fitting the beam and atmospheric muon neutrino samples are described in the following sections.

\subsubsection{Beam Systematic Uncertainties}

The fit minimizes the statistic using the four dominant beam uncertainties: normalization, NC background, shower energy, and track energy. The beam systematic uncertainties are completely correlated between all MINOS and MINOS+ runs. Except for the NC background uncertainties, all the uncertainties are the same between MINOS and MINOS+.

\section{Normalization}

The beam normalization uncertainty quantifies differences between the ND and the FD that would affect a FD prediction by a normalization factor. The largest contribution to the uncertainty comes from differences in event 
selection and reconstruction between the two detectors. Other contributions come from uncertainties on the fiducial mass and live-time of each detector. This uncertainty was estimated by comparing selection and reconstruction of $\mathrm{MC}$ and data for each detector done by software and hand-scanning. The study took into account the efficiencies of identifying tracks, charge $\left(\mu^{-}\right.$or $\mu^{+}$), and interaction vertices in the fiducial volume $[110,111]$. The uncertainty estimation was improved in Ref. [112]. The current normalization uncertainty is $1.6 \%$ for both MINOS and MINOS+.

\section{NC Background}

For MINOS, the NC background was evaluated using two methods. The first method estimates the uncertainty by comparing the spectrum of events that fail the $\mathrm{CC}$ selection for data and $\mathrm{MC}$. The events that fail the CC selection should be mainly populated by NC interactions. The uncertainty from the first method is taken as the ratio of the data spectrum to the MC spectrum. The second method estimates the uncertainty using samples of selected $\mathrm{CC}$ events with the muon track algorithmically removed to produce events that contain only showers. This procedure is done for both MC and data. The Muon Removed CC (MRCC) samples imitate NC interactions in the detector. The value of the uncertainty is determined by the ratio of the number of MRCC events from data to the number of MRCC events from MC that pass the CC selection [113].

For MINOS, this systematic uncertainty was evaluated at $20 \%$ by sum- 
ming the uncertainties from the above methods in quadrature. This uncertainty was reevaluated for MINOS+ using only the MRCC method. The MINOS+ uncertainty was found to be $50 \%$ [114].

\section{Shower Energy}

The shower energy uncertainty has an energy dependent component that comes from uncertainties in modeling hadronic interactions [115]. An additional flat 5.7\% uncertainty comes from comparisons of data and MC [116]. The final functional form of the shower energy uncertainty is:

$$
\sigma_{\text {shower }}=6.6 \%+3.5 \% \exp \left(-E_{\text {shower }} / 1.44 \mathrm{GeV}\right) \text {, }
$$

where $E_{\text {shower }}$ is the reconstructed shower energy.

\section{Track Energy}

The track energy uncertainty quantifies the overall uncertainty on track energy reconstructed from range or curvature. The uncertainties are treated together since the curvature energy uncertainty is derived from the range energy uncertainty, making them perfectly correlated. The range method of track energy calculation, which is applied to fully contained tracks, has a $2 \%$ uncertainty [117]. This uncertainty comes from uncertainties on the detector mass, detector geometry in the reconstruction software, and the energy loss model used [50]. The curvature method of track energy calculation, which is applied to tracks that exit the detector or terminate in the magnetic coil, differs from the track method value by $1 \%$. The total uncertainty on the curvature energy 
calculation is $3 \%$ from the range uncertainty added in quadrature with the $1 \%$ uncertainty between the two methods [117].

\subsubsection{Atmospheric Systematic Uncertainties}

The atmospheric neutrino measurement uncertainties are significantly affected by uncertainties in the atmospheric neutrino flux and neutrino cross sections. Since the Bartol 3D flux model [104] is used to predict the atmospheric flux, the flux uncertainties primarily come from the Bartol group's study of flux uncertainties [118]. Additional flux uncertainties come from com-

paring the Bartol 3D model to the Fluka 3D [119] and Honda 3D [120] models. The uncertainties can be divided into three categories based on their effects: normalization, shape, and energy scale. The uncertainties are discussed in detail in Ref. [121] and summarized below.

\section{CV normalization}

The normalization uncertainties come from uncertainties on the flux and the cross section. The flux uncertainties were studied by the Bartol group in Ref. [118]. In the neutrino energy region, $1 \mathrm{GeV}$ to $10 \mathrm{GeV}$, relevant for $\mathrm{CV}$ events, the flux uncertainty is $15 \%$. The cross section uncertainty peaks at $\approx 8 \%$ for neutrinos with energies from $1 \mathrm{GeV}$ to $5 \mathrm{GeV}$. The $\mathrm{CV}$ normalization uncertainty is chosen as $15 \%$ to cover these flux and cross section uncertainties. 


\section{Rock normalization}

Non-fiducial neutrinos are created by cosmic rays on other side of Earth with a median energy of $50 \mathrm{GeV}$ and up to $1 \mathrm{TeV}$. Following Ref. [118], the flux uncertainty continues to increase after $10 \mathrm{GeV}$. For neutrino energies $>100 \mathrm{GeV}$, the uncertainty is $>25 \%$. Compared to the lower energy CV events, the cross section of these high energy neutrinos is accurately measured with an uncertainty of $3 \%$. The rock normalization uncertainty is set to $25 \%$ to cover these uncertainties.

\section{CV charge}

The charge uncertainty is the uncertainty on the ratio of $\bar{v}_{\mu}$ to $v_{\mu}$. This uncertainty has three components: the relative flux uncertainty, the relative cross section uncertainty, and a reconstructed charge purity uncertainty.

As with the normalization uncertainties, the relative flux uncertainty is determined based on the studies by the Bartol group. The relative flux uncertainty for $\mathrm{CV}$ events is conservatively calculated to be $4 \%$ based on these studies.

The relative cross section uncertainty was derived from studies of varying the inputs into the NEUGEN cross section model within their uncertainties. This is the largest uncertainty for the CV charge ratio at $8.5 \%$.

The uncertainty on the purity of the reconstructed charge is evaluated by hand-scanning data and MC. The uncertainty is chosen to cover differences 
between data and $\mathrm{MC}$ as well as the differences between reconstruction and truth for MC. For CV events, the uncertainty on the charge purity is determined to be $2 \%$. This value translates to a $3 \%$ uncertainty on the CV charge ratio.

These three uncertainties are added in quadrature to get a CV charge uncertainty of $10 \%$.

\section{Rock charge}

The rock event charge uncertainty is the uncertainty on the ratio of $\bar{v}_{\mu}$ to $v_{\mu}$ for upward-going neutrinos. This uncertainty is determined using the same procedure as the CV charge uncertainty.

The relative flux uncertainty is set to $10 \%$. This uncertainty sufficiently covers flux differences between the Bartol 3D model [104] and the Fluka 3D [119] and Honda 3D [120] models at the higher energies relevant for non-fiducial neutrino events.

The relative cross section is well measured in the region relevant for non-fiducial neutrino events. Thus, an uncertainty of $4 \%$ is used.

As with the CV events, the charge purity of the non-fiducial events was studied and a $4 \%$ uncertainty in charge purity was necessary to cover differences between data and MC. This $4 \%$ uncertainty in charge purity becomes a $6 \%$ uncertainty on the charge ratio.

The result of adding the above uncertainties in quadrature is a $12.5 \%$ 
rock charge uncertainty.

\section{$v_{\mathrm{e}}$ normalization and NC background}

The $v_{\mathrm{e}}$ normalization uncertainty is the uncertainty on the ratio of $v_{\mathrm{e}}$ events to $v_{\mu}$ events. This uncertainty is also related to the $\mathrm{NC}$ background uncertainty, which is the uncertainty on the ratio of $\mathrm{NC}$ to $\mathrm{CC}$ events, since they both depend on uncertainties modeling hadronic interactions.

The hadronic modeling uncertainty was evaluated by comparing the shower event rates between GCALOR [122] and GHEISHA [123]. The comparison of the models found a $7 \%$ difference in rate. This difference was further divided into $v_{\mathrm{e}}$ showers and $\mathrm{NC}$ interactions.

The resulting $v_{\mathrm{e}}$ uncertainty is $5 \%$ [121]. The NC background uncertainty carries an additional contribution from the ratio of the NC cross section to the CC cross section, which is large compared to the hadronic modeling uncertainty. The combined hadronic modeling and cross section uncertainties result in an NC background uncertainty of $20 \%$ [121].

\section{Spectrum uncertainties}

The predicted atmospheric $v_{\mu}$ and $\bar{v}_{\mu}$ spectra are allowed to be scaled according to Eq. 6.14 to account for uncertainties in the shape of the spectra.

$$
f\left(E_{v}\right)= \begin{cases}1+\alpha\left(E_{v}-E_{0}\right), & E_{v} \leq E_{0} \\ 1+\alpha \ln \left(E_{v} / E_{0}\right), & E_{v}>E_{0}\end{cases}
$$

The scaling function is constructed to account for the predicted spectrum ap-

proximately following a power-law at high neutrino energies. At the threshold 
energy, $E_{0}$, the high energy form is smoothly connected to a linear function. In the analysis, $E_{0}=3 \mathrm{GeV}$ which is approximately the median energy of the events with well-measured direction. The spectrum parameter, $\alpha$, is defined to follow a Gaussian distribution centered at zero. The uncertainty on $\alpha, \sigma_{\alpha}$, is defined separately for neutrinos and antineutrinos to cover the spectrum shape uncertainties from the flux prediction and cross section model. The flux and cross section uncertainties are evaluated separately and then added in quadrature to obtain $\sigma_{\alpha}$.

For the $v_{\mu}$ flux, $\sigma_{\alpha}^{\text {flux }}=5 \%$ covers the differences between the Bartol 3D model [104] and the Fluka 3D [119] and Honda 3D [120] models when the flux at the threshold energy is normalized to 1 for each model. The shape uncertainty from the cross section was studied by comparing the nominal cross section to the cross section derived when the the quasi-elastic axial-vector mass was varied by $\pm 15 \%$. The cross section uncertainty, $\sigma_{\alpha}^{\mathrm{X}}=3 \%$, covers the cross section ratios when the value at the threshold energy is normalized to 1 . The final uncertainty for the $v_{\mu}$ spectrum parameter is then $\sigma_{\alpha}=6 \%$.

The same procedure was used to evaluate the uncertainty on the $\bar{v}_{\mu}$ spectrum parameter. The component uncertainties were set to $\sigma_{\alpha}^{\text {flux }}=6 \%$ and $\sigma_{\alpha}^{\mathrm{X}}=2 \%$. These uncertainties added in quadrature yield the total uncertainty of $\sigma_{\alpha}=6 \%$.

Thus, the spectrum uncertainty is set to $6 \%$ for muon neutrinos and muon antineutrinos for both the CV and non-fiducial events. 


\section{Zenith}

The zenith uncertainty is the uncertainty on the ratio of upward traveling events to downward traveling events. This uncertainty is comprised of uncertainties of the flux ratio and uncertainties that come from the purity and efficiency of direction reconstruction. A 3\% uncertainty covers the effects of the underlying uncertainties.

\section{Shower energy}

The shower energy uncertainty for atmospheric events is the result of adding in quadrature the uncertainties from hadronization modeling, detector calibration, and shower energy reconstruction.

The hadronization uncertainty is taken from the beam analysis [115] and is rounded up to $10 \%$. The calibration uncertainty evaluated in Ref. [124] is increased to $5 \%$. The shower energy reconstruction uncertainty was determined to be $10 \%$.

After combining the above uncertainties, the shower energy uncertainty is $15 \%$.

\section{Track energy uncertainties}

The uncertainty on track energy determined by range is set to $3 \%$ for atmospheric neutrino events. The uncertainty on the track energy calculated from the track curvature was determined by comparing the range and curvature energy measurements for stopping muons. The mean calculated energy 
values for each method were found to agree within $3 \%$ with data and MC. The uncertainty on the track energy from curvature is conservatively set at $5 \%$ to cover the differences between the two methods as well as the differences between data and MC.

\subsubsection{Correlated Systematics}

When the MINOS and MINOS+ beam data sets are fit together, the beam systematic uncertainties are treated as perfectly correlated between the two periods. However, making this same assumption for the NC background uncertainties is not well motivated since different event selector are used MINOS and MINOS+. The effect of shifting the beam systematic uncertainties

on the best fit of $\Delta m_{32}^{2}$ and $\sin ^{2} \theta_{23}$ when they are correlated and uncorrelated is shown in Fig. 6.10. From the study of shifting the MINOS and MINOS+ uncertainties in an uncorrelated manner, the MINOS+ NC background was found to have a negligible effect on the overall best fit point. Thus, the MINOS+ NC background is neglected when the MINOS and MINOS+ beam data set are fit together.

When the atmospheric neutrino data set is included in the fit with beam data, the atmospheric neutrino track energy from range and shower energy uncertainty are correlated with the beam uncertainties. The atmospheric track energy uncertainty is assumed to be perfectly correlated with the beam overall track energy systematic. The atmospheric shower energy uncertainty is correlated with the beam shower energy systematic since both are derived 

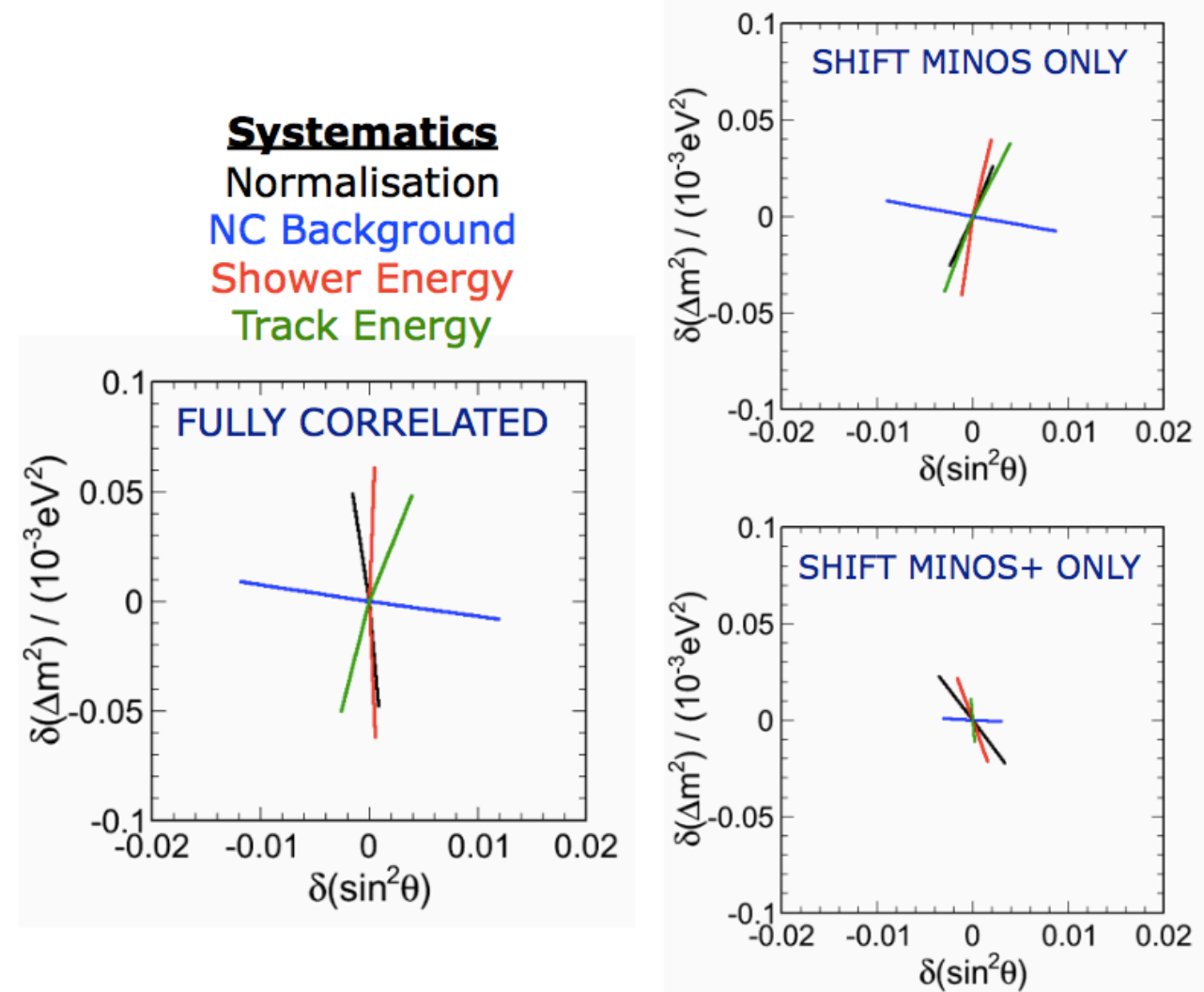

Figure 6.10: The effects of altering the neutrino spectrum by shifting the beam systematic uncertainties by $\pm 1 \sigma$ on the statistics only fit of $\Delta m_{32}^{2}$ and $\sin ^{2} \theta_{23}$ for the combined MINOS and MINOS+ neutrino beam exposure. The left plot shows the effects of shift the MINOS and MINOS+ spectra together with fully correlated systematics. The top plot shows the effects of only systematically shifting the MINOS spectrum, and the bottom plot shows the effects of only systematically shifting the MINOS+ spectrum. From Ref. [125]. 
from a common hadronization model uncertainty [126].

\subsubsection{Summary of Systematic Uncertainties}

The beam sample systematic uncertainties for MINOS and MINOS+ are summarized in Table 6.4, and the atmospheric sample systematic uncertainties are summarized in Table 6.5. Figure 6.11 shows the effect of shifting the neutrino spectrum by $\pm 1 \sigma$ of the beam and atmospheric systematic uncertainties on the best fit of $\Delta m_{32}^{2}$ and $\sin ^{2} \theta_{23}$ for a statistics only fit. The plots in Fig. 6.11 show that all four beam systematic uncertainties have comparable effects and that the CV and rock normalization atmospheric uncertainties produce the largest shifts of the atmospheric uncertainties. The best fit systematic uncertainty of values from the fit to the combined MINOS and MINOS+ beam and atmospheric data samples are summarized in Table 6.6.

Table 6.4: MINOS and MINOS+ beam sample $1 \sigma$ systematic uncertainty values.

\begin{tabular}{lr}
\hline Systematic & $\%$ \\
\hline normalization & 1.6 \\
NC background & 50 \\
shower energy & $6.6+3.5 \exp \left(-E_{\text {shower }} / 1.44 \mathrm{GeV}\right)$ \\
track energy (range) & 2 \\
track energy (curvature) & 3 \\
\hline
\end{tabular}


Table 6.5: Atmospheric neutrino sample $1 \sigma$ systematic uncertainty values.

\begin{tabular}{|c|c|c|}
\hline Systematic & Description & $\%$ \\
\hline CV normalization & normalization of $\mathrm{CV} v_{\mu}$ events & 15 \\
\hline rock normalization & normalization of non-fiducial $v_{\mu}$ events & 25 \\
\hline CV charge & $\mathrm{CV} \bar{v}_{\mu} / v_{\mu}$ ratio & 10 \\
\hline rock charge & non-fiducial $\bar{v}_{\mu} / v_{\mu}$ ratio & 12.5 \\
\hline$v_{\mathrm{e}}$ normalization & $\mathrm{CV} v_{\mathrm{e}} / v_{\mu}$ ratio & 5 \\
\hline NC background & $\mathrm{NC} / \mathrm{CC}$ ratio & 20 \\
\hline$v_{\mu} \mathrm{CV}$ spectrum & & 6 \\
\hline $\bar{v}_{\mu} \mathrm{CV}$ spectrum & & 6 \\
\hline$v$ rock spectrum & & 6 \\
\hline $\bar{v}$ rock spectrum & & 6 \\
\hline zenith & up/down ratio & 3 \\
\hline shower energy & & 15 \\
\hline track energy (range) & & 3 \\
\hline track energy (curvature) & & 5 \\
\hline
\end{tabular}



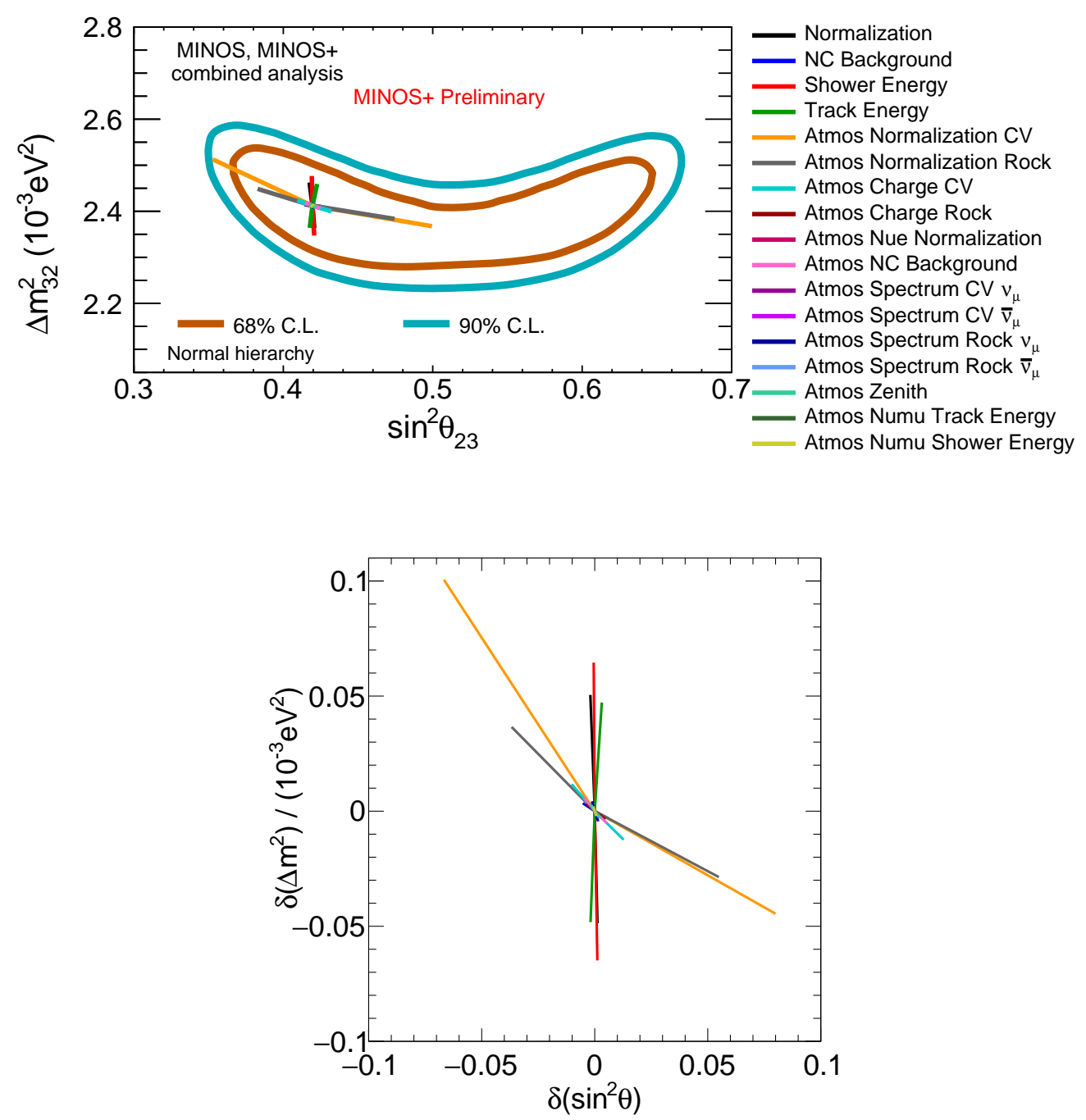

Figure 6.11: The effects of altering the neutrino spectrum by shifting the beam and atmospheric systematic uncertainties by $\pm 1 \sigma$ on the statistics only fit of $\Delta m_{32}^{2}$ and $\sin ^{2} \theta_{23}$. The top plot shows the resulting shifts compared to the $2 \mathrm{D}$ confidence intervals in $\Delta m_{32}^{2}$ and $\sin ^{2} \theta_{23}$ for the combined MINOS and MINOS+ beam and atmospheric data samples. The bottom plot shows the relative changes in $\Delta m_{32}^{2}$ and $\sin ^{2} \theta_{23}$ from the best fit value as a result of shifting the systematic uncertainties. 
Table 6.6: MINOS and MINOS+ beam and atmopsheric systematic uncertainty best fit values, accounting for correlations.

\begin{tabular}{lr}
\hline Systematic & Best Fit Value $(\sigma)$ \\
\hline normalization & 1.41 \\
NC background & 0.17 \\
shower energy & -0.38 \\
correlated track energy & 0.62 \\
CV normalization & 0.45 \\
rock normalization & 0.16 \\
CV charge & -0.68 \\
rock charge & 1.19 \\
$v_{\mathrm{e}}$ normalization & -0.12 \\
NC background & -0.24 \\
$\boldsymbol{v}_{\mu}$ CV spectrum & -0.73 \\
$\bar{v}_{\mu}$ CV spectrum & 1.15 \\
$v_{\text {rock spectrum }}$ & 0.20 \\
$\bar{v}$ rock spectrum & 0.11 \\
zenith & -0.14 \\
shower energy & 0.14 \\
atm. track energy (curvature) & -0.27 \\
\hline
\end{tabular}

\subsection{Measuring $\Delta m_{32}^{2}$ and $\sin ^{2} \theta_{23}$}

The measurement of $\Delta m_{32}^{2}$ and $\sin ^{2} \theta_{23}$ is done by performing the fit in the $\Delta m_{32}^{2}-\sin ^{2} \theta_{23}$ plane to produce a $\chi^{2}$ surface. A separate surface is made for each mass hierarchy. Figures 6.12 and 6.13 show the $\chi^{2}$ surfaces for the combined fit of all MINOS and MINOS+ beam and atmospheric data. In order to constrain the oscillation parameters, the global minimum $\chi^{2}$ value from the $\chi^{2}$ surfaces is subtracted from each point to form $\Delta \chi^{2}$ surfaces. The $\Delta \chi^{2}$ surfaces are then used to make 2D and $1 \mathrm{D}$ confidence limits assuming that the 


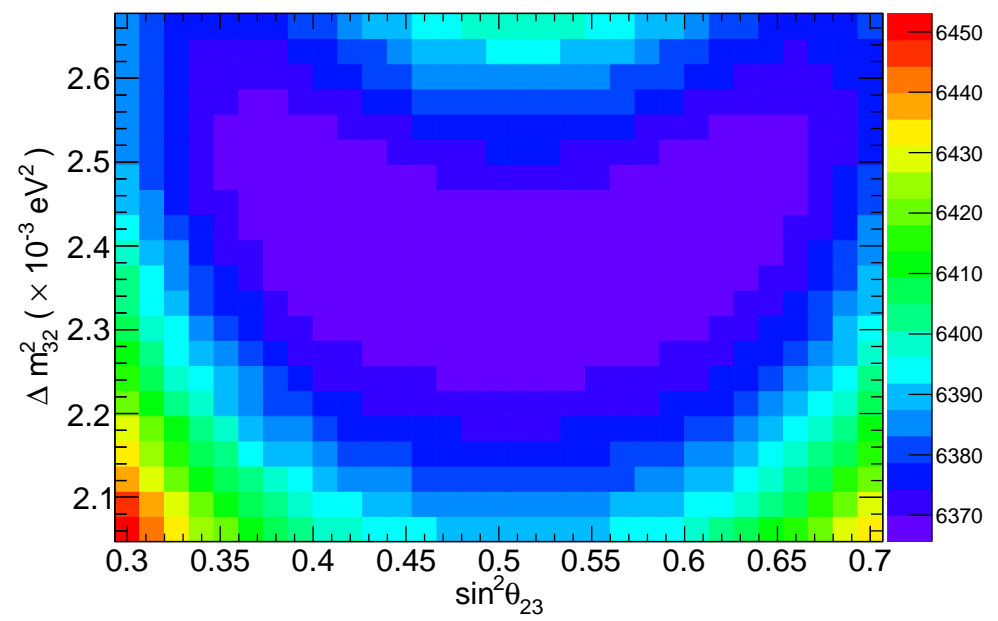

Figure 6.12: $\chi^{2}$ surface produced by the MINOS, MINOS + fit for the normal mass hierarchy.

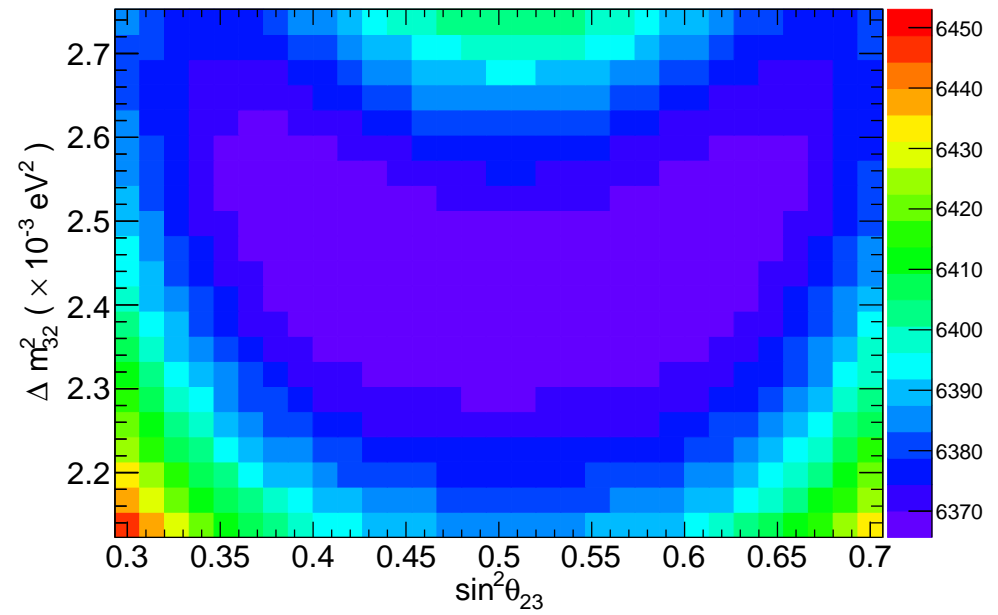

Figure 6.13: $\chi^{2}$ surface produced by the MINOS, MINOS+ fit for the inverted mass hierarchy. 
$\Delta \chi^{2}$ values obey a Gaussian distribution. The constraints on the individual oscillation parameters come from the 1D confidence limits, also referred to as profiles. 


\section{Chapter 7}

\section{Results}

This work adds the analysis of the final two years of MINOS+ beam exposure in order to do a three-flavor fit using all available MINOS and MINOS+

data, including significantly more events above the first oscillation maximum, allowing the model to be tested with more precision. The results from jointly fitting the MINOS+ Runs 12 and 13 are consistent with the muon neutrino disappearance measured by MINOS [64] and summarized in Appendix A. This chapter summarizes the three-flavor neutrino oscillation fits to MINOS+; the combined MINOS, MINOS+ samples; and a preliminary combined fit using data from the NOvA experiment.

\subsection{MINOS+}

During MINOS+ running from 2013-2016, $6280 \nu_{\mu} \mathrm{CC}$ and $293 \bar{v}_{\mu} \mathrm{CC}$ contained-vertex events are measured at the FD, compared to $7300 \nu_{\mu} \mathrm{CC}$ and $302 \bar{v}_{\mu}$ CC predicted by the no oscillations hypothesis. The $v_{\mu}$ and $\bar{v}_{\mu}$ event counts and predictions for the full MINOS+ beam exposure are available in Appendix B, Tables B.1 and B.2.

The measured MINOS + spectrum of $v_{\mu}$ and $\bar{v}_{\mu}$ contained-vertex CC 
events is compared to the predicted spectra with and without oscillations in Fig. 7.1. The data and best fit spectra are consistent with the best fit measured using the full MINOS data set in Ref. [64], showing negligible differences between the two best fit predictions. Large differences between the best fit predictions in the region of 4 to $10 \mathrm{GeV}$ would indicate an effect not in the three-flavor model. The ratio of data to the no oscillations prediction in

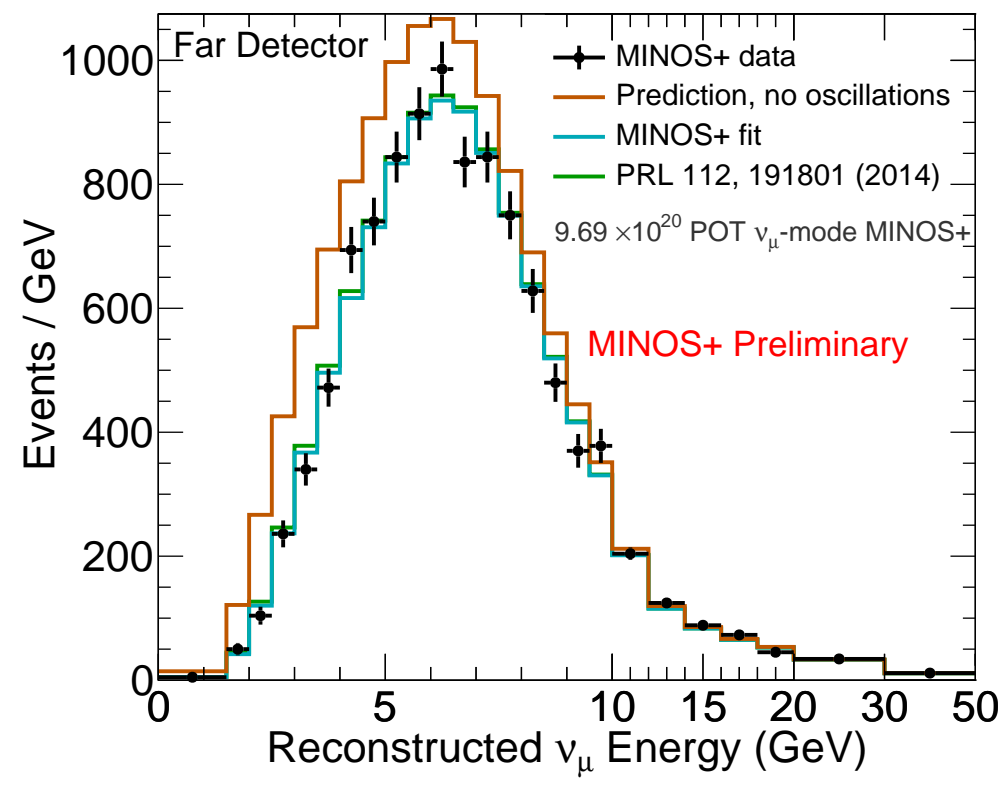

Figure 7.1: MINOS+ contained-vertex $v_{\mu}$ and $\bar{v}_{\mu}$ data spectrum and predictions at the far detector. The data spectrum is shown as black points. The orange curve is the predicted spectrum with no oscillations, and the best fit spectrum is shown in blue for $\Delta m_{32}^{2}=-2.51 \times 10^{-3} \mathrm{eV}^{2}$ and $\sin ^{2} \theta_{23}=0.62$. The predicted spectrum assuming the best fit measured using the full MINOS data set [64] is shown in green.

Fig. 7.2 clearly shows muon neutrino disappearance despite the beam neutrinos predominantly having energies above the oscillation maximum. 


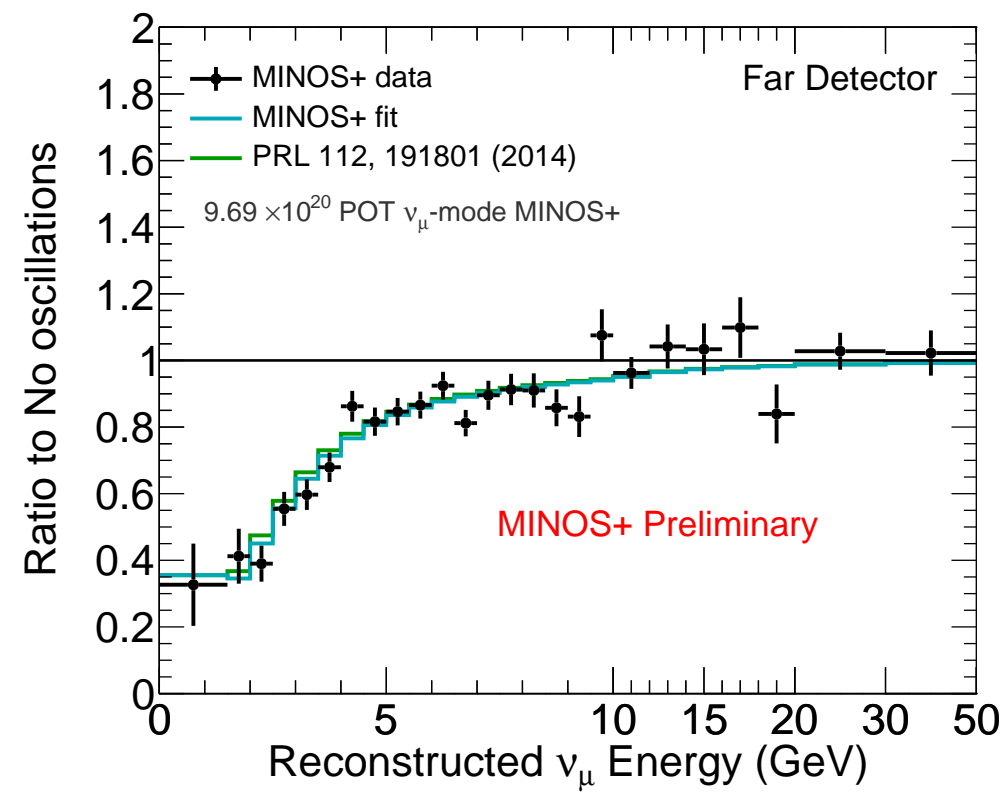

Figure 7.2: MINOS+ ratios of data and best fit spectra to the no oscillations prediction. The data ratio in black clearly shows muon neutrino disappearance. The green curve is the ratio for the prediction assuming the MINOS best fit from Ref. [64]. The ratio for the best fit at $\Delta m_{32}^{2}=-2.51 \times 10^{-3} \mathrm{eV}^{2}$ and $\sin ^{2} \theta_{23}=0.62$ is drawn in blue.

An additional test of agreement with the three-flavor model is done by checking that the fit limits contain the global best fit values. The $\Delta m_{32}^{2}$ and $\sin ^{2} \theta_{23}$ confidence limits for both mass hierarchies using the full MINOS+ beam exposure in Fig. 7.3 indeed contain the global best fit values for within the $68 \%$ C.L., building confidence that the data are described by three-flavor oscillations. The best fit oscillation parameters for the MINOS+ data are $\sin ^{2} \theta_{23}=0.62$ and $\Delta m_{32}^{2}=-2.51 \times 10^{-3} \mathrm{eV}^{2}$. Since the minimum $-2 \Delta \log (\mathcal{L})$ values for the 1D limits in Fig. 7.3 are indistinguishable, the data are consistent 
with either of the mass hierarchies. Although the fit has a preference for nonmaximal mixing as seen by the increase in $-2 \Delta \log (\mathcal{L})$ around $\sin ^{2} \theta_{23}=0.5$, the difference is insignificant, and the maximal mixing point is well within the $68 \%$ confidence limit. Furthermore, there is no preference for either octant of $\theta_{23}$.
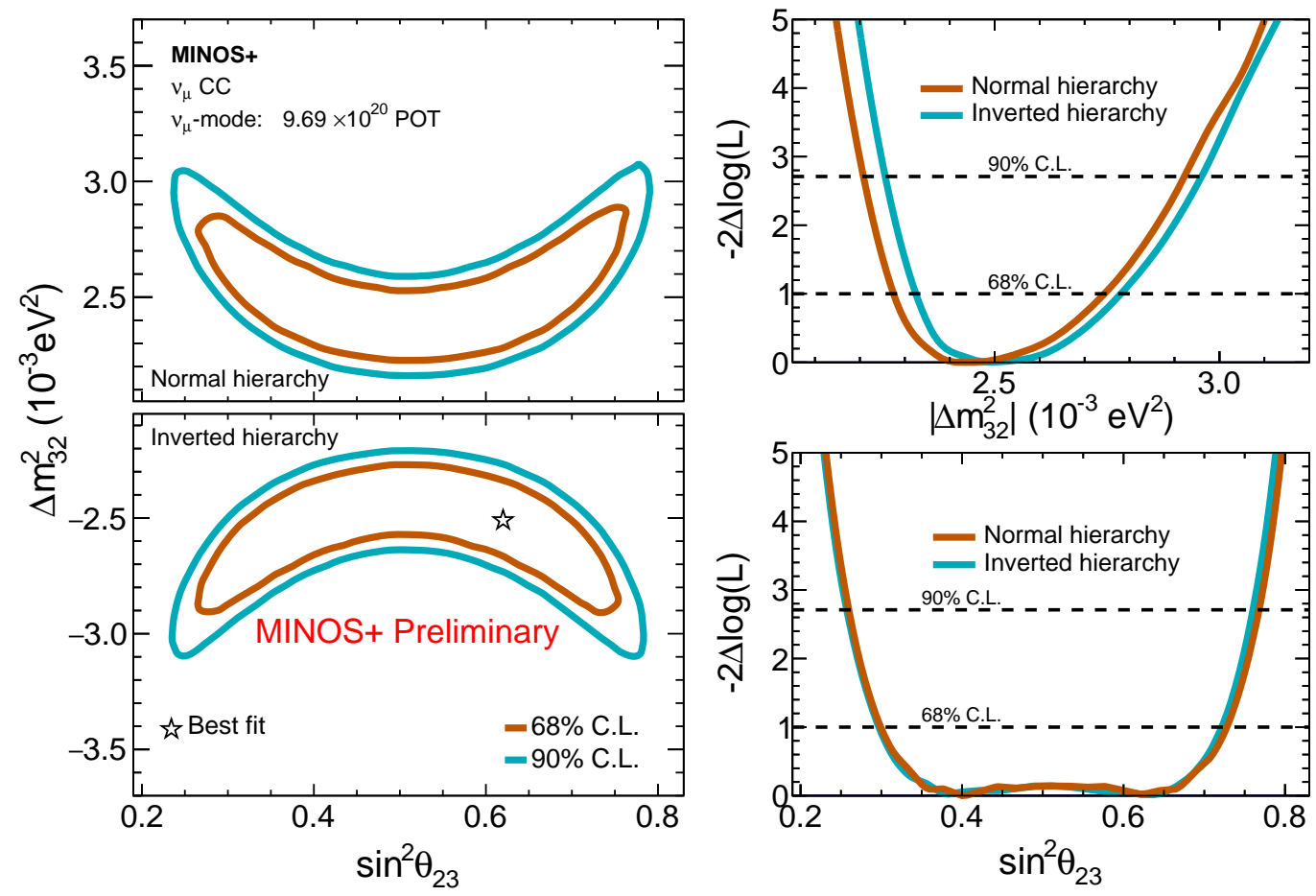

Figure 7.3: MINOS $+\Delta m_{32}^{2}$ and $\sin ^{2} \theta_{23}$ confidence limits. The left panel shows the $68 \%$ and $90 \%$ confidence limits on $\Delta m_{32}^{2}$ and $\sin ^{2} \theta_{23}$ for the normal mass hierarchy (top) and the inverted mass hierarchy (bottom). The best fit from the full MINOS+ beam exposure is plotted as a star. The right panels show the 1D likelihood profiles as functions of $\Delta m_{32}^{2}$ and $\sin ^{2} \theta_{23}$ for each mass hierarchy.

Tables B.3 to B.6 in Appendix B summarize the fit preferences and 
limits for the MINOS+ data from 2013-2016. The MINOS+ data are consistent with the results from MINOS [64], and the confidence limits agree with the limits from MINOS. With the full MINOS+ exposure, MINOS+ measures $\left|\Delta m_{32}^{2}\right|=2.47_{-7.7 \%}^{+10.9 \%} \times 10^{-3} \mathrm{eV}^{2}(68 \%$ C.L. $)$ for the normal mass hierarchy and $\left|\Delta m_{32}^{2}\right|=2.51_{-7.2 \%}^{+9.7 \%} \times 10^{-3} \mathrm{eV}^{2}(68 \%$ C.L. $)$ for the inverted mass hierarchy.

\subsection{MINOS and MINOS+}

In total, MINOS observed 6028 beam events compared to 7223 expected in the case of no oscillations. MINOS+ observed 6573 beam events compared to 7671 expected in the case of no oscillations. The atmospheric neutrino sample contains 3237 events compared to 4040 expected in the case of no oscillations. Table 7.1 summarizes the number of selected events from the MINOS and MINOS+ data sets and compares the observed number of events to the predictions from the best fit and no oscillations case. Tables C.1 to C.6 in Appendix $\mathrm{C}$ provide a detailed breakdown of the event counts observed and predicted by selected event type.

MINOS measured $2579 \boldsymbol{v}_{\mu}$ CC events and $312 \bar{v}_{\mu}-\mathrm{CC}$ events in $\boldsymbol{v}_{\mu^{-}}$ mode. MINOS+ measured $6280 \boldsymbol{v}_{\mu}-\mathrm{CC}$ events and $293 \overline{\boldsymbol{v}}_{\mu^{-}} \mathrm{CC}$ events in $\boldsymbol{v}_{\mu^{-}}$ mode. The fact that MINOS+ recorded 3701 more $v_{\mu}$-CC interactions than MINOS despite having $90.5 \%$ the exposure as MINOS in $\boldsymbol{v}_{\mu}$-mode is due in part to the neutrino cross section scaling with energy and that the MINOS+ neutrino beam peaks away from the oscillation maximum.

When the MINOS+ data are considered in combination with the com- 
Table 7.1: Number of events selected in each sample for the combined MINOS and MINOS+ data compared to the no oscillations and best fit predictions.

\begin{tabular}{lrrr}
\hline Sample & No Osc. & Best Fit & Observed \\
\hline $\boldsymbol{v}_{\mu}$ from $\boldsymbol{v}_{\mu}$ beam & 10641 & 8849 & 8859 \\
$\bar{\nu}_{\mu}$ from $\boldsymbol{v}_{\mu}$ beam & 676 & 598 & 605 \\
Nonfiducial $\mu$ from $\nu_{\mu}$ beam & 3257 & 2839 & 2911 \\
$\bar{\nu}_{\mu}$ from $\bar{v}_{\mu}$ beam & 320 & 225 & 226 \\
Atm. contained-vertex $\nu_{\mu}+\bar{v}_{\mu}$ & 1884 & 1366 & 1378 \\
Atm. nonfiducial $\mu^{-}+\mu^{+}$ & 933 & 735 & 736 \\
Atm. showers & 1223 & 1130 & 1123 \\
\hline
\end{tabular}

plete MINOS beam exposure, the combined spectrum covers a large energy range that spans the maximum disappearance to the maximum survival probability of the muon neutrinos. Figure 7.4 shows the total measured beam spectrum from neutrino events compared to the no oscillations prediction and best fit prediction using the entire MINOS and MINOS+ data set.

The ratio of the full MINOS and MINOS+ neutrino spectrum to the unoscillated prediction is shown in black in Fig. 7.5. It clearly shows muon neutrino disappearance with the maximum disappearance occurring between 1 and $2 \mathrm{GeV}$.

\subsubsection{Measurement of $\Delta m_{32}^{2}$ and $\sin ^{2} \theta_{23}$}

Figure 7.6 compares the $\Delta m_{32}^{2}$ and $\sin ^{2} \theta_{23}$ contours before and after adding the MINOS+ data and $12.08 \mathrm{kt} \cdot \mathrm{yr}$ of atmospheric neutrino data. The best fit using the full data set, $\Delta m_{32}^{2}=2.41 \times 10^{-3} \mathrm{eV}^{2}$ and $\sin ^{2} \theta_{23}=0.42$, is 


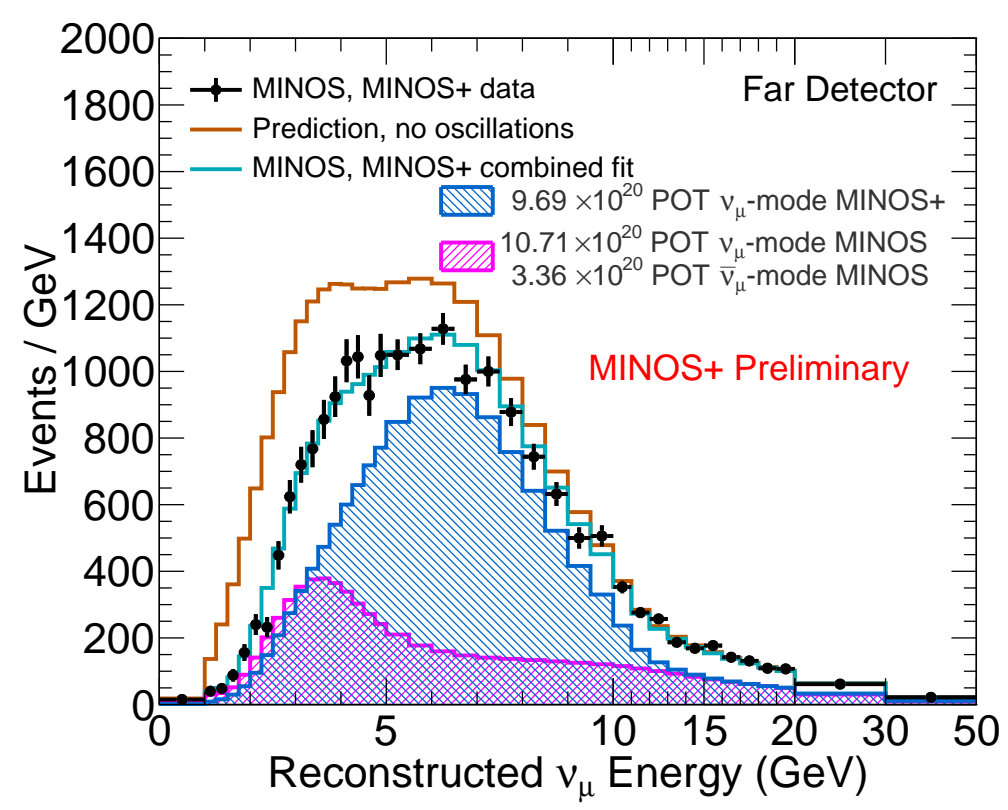

Figure 7.4: MINOS and MINOS+ combined contained-vertex $\nu_{\mu}$ and $\bar{v}_{\mu}$ data spectrum and predictions at the far detector. The data spectrum is shown as black points. The orange curve is the predicted spectrum with no oscillations, and the blue curve is the best fit to the data for $\Delta m_{32}^{2}=2.41 \times 10^{-3} \mathrm{eV}^{2}$ and $\sin ^{2} \theta_{23}=0.42$. The best fit prediction is broken down into the MINOS best fit spectrum (hatched pink) and MINOS+ best fit spectrum (hatched blue).

contained by both sets of contours.

The full data set has a negligible preference for the normal mass hierarchy which can be seen in Fig. 7.7 by the separation of the 1D profiles in $-2 \Delta \log (\mathcal{L}) . \quad$ In $\sin ^{2} \theta_{23}$ the normal mass hierarchy and inverted mass hierarchy look similar to each other. At the $68 \%$ confidence limit non-maximal mixing is preferred, and the best fit for $\sin ^{2} \theta_{23}$ in both mass hierarchies prefers $\theta_{23}<\pi / 4$. 


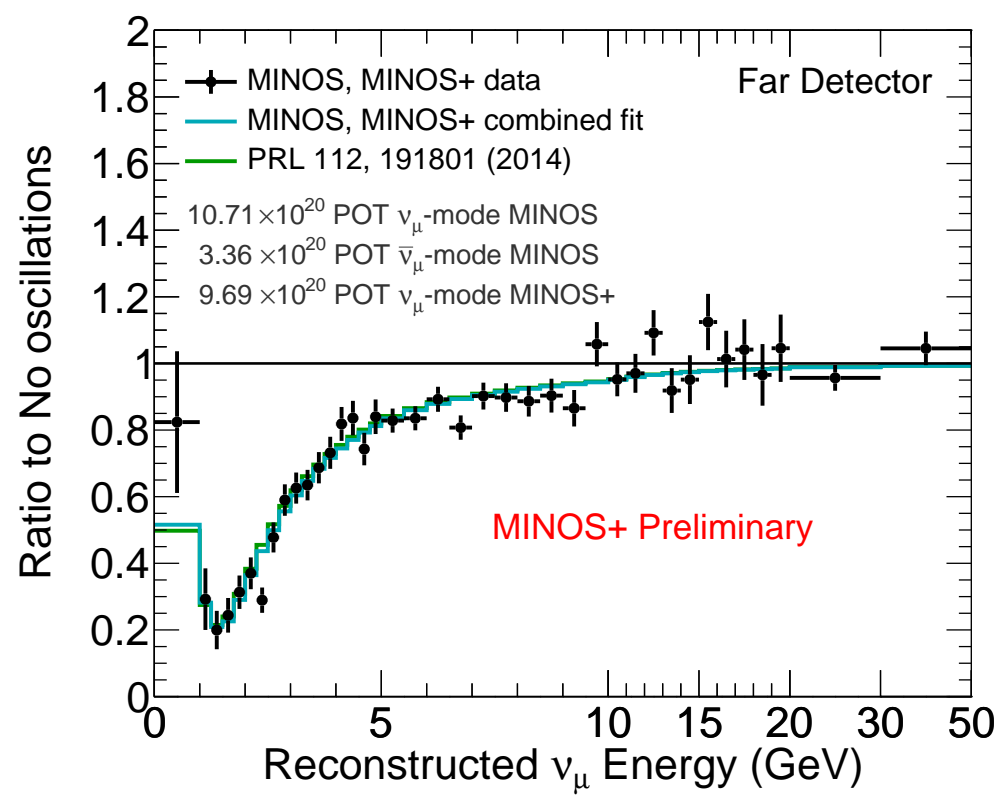

Figure 7.5: MINOS and MINOS+ combined ratios of data and best fit spectra to the no oscillations prediction. The data ratio in black clearly shows muon neutrino disappearance. The green curve is the ratio for the prediction assuming the MINOS best fit from Ref. [64]. The ratio for the best fit at $\Delta m_{32}^{2}=2.41 \times 10^{-3} \mathrm{eV}^{2}$ and $\sin ^{2} \theta_{23}=0.42$ is drawn in blue.

The combined data set, including the atmospheric neutrino exposure and the MINOS $v_{\mathrm{e}}$-appearance constraint, measures $\Delta m_{32}^{2}=2.41 \pm 3.7 \% \times$ $10^{-3} \mathrm{eV}^{2}\left(68 \%\right.$ C.L.) and $\sin ^{2} \theta_{23}=0.36-0.65$ (90\% C.L.) for the normal mass hierarchy and $\Delta m_{32}^{2}=2.47_{-4.0 \%}^{+3.2 \%} \times 10^{-3} \mathrm{eV}^{2}(68 \%$ C.L. $)$ and $\sin ^{2} \theta_{23}=0.36-0.65$ (90\% C.L.) for the inverted mass hierarchy.

Table 7.2 lists the best fit points and $-2 \Delta \log (\mathcal{L})$ from the global minimum for each mass hierarchy and octant of $\theta_{23}$ for all of the MINOS and MINOS+ data. The fit mass hierarchy and $\sin ^{2} \theta_{23}$ confidence limits and pref- 


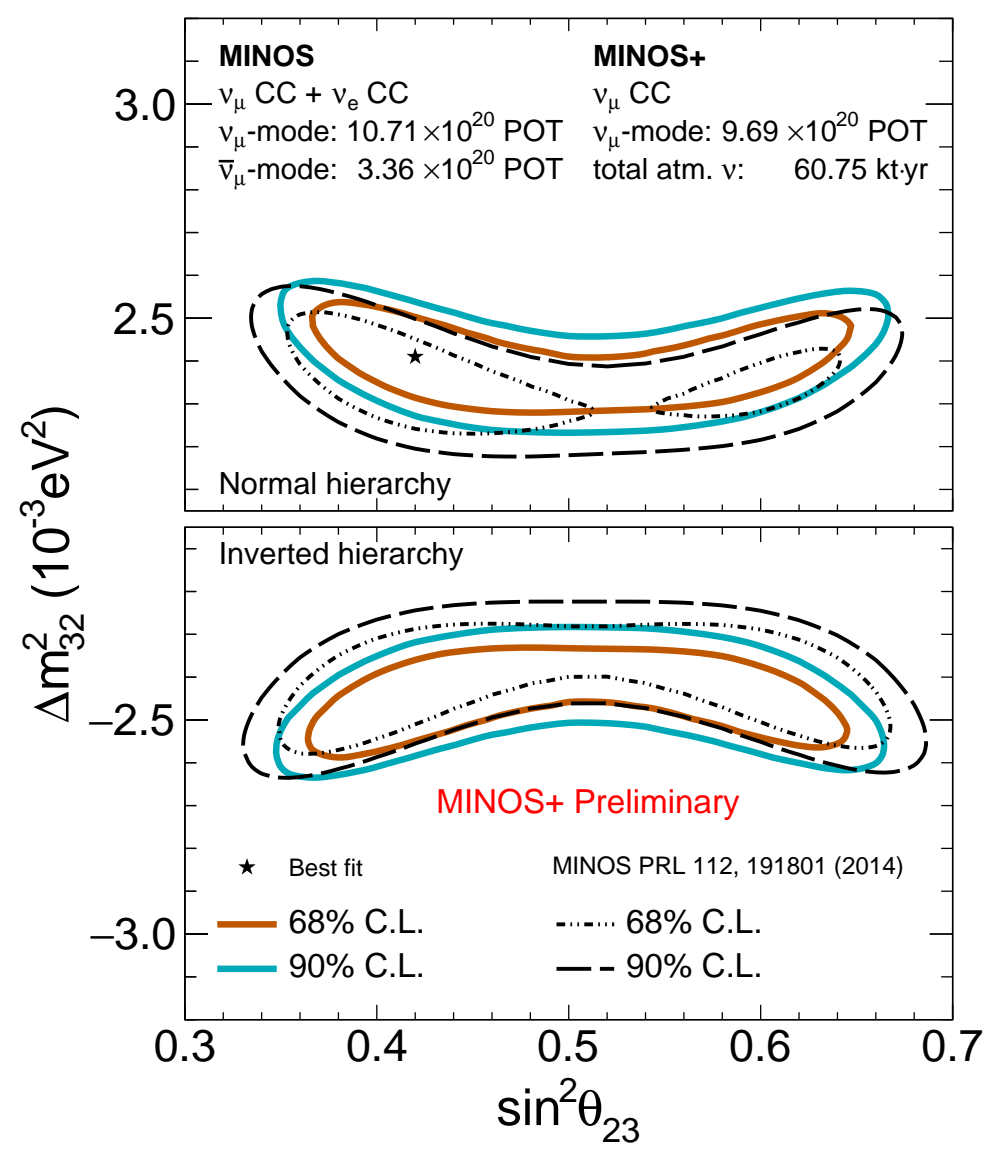

Figure 7.6: MINOS, MINOS+ combined contours in $\Delta m_{32}^{2}$ and $\sin ^{2} \theta_{23}$ compared to the MINOS contours [64]. The panel shows the $68 \%$ and $90 \%$ confidence limits on $\Delta m_{32}^{2}$ and $\sin ^{2} \theta_{23}$ for the normal mass (top) and the inverted mass hierarchy (bottom). The best fit from the full MINOS and MINOS+ exposure is plotted as a star. 

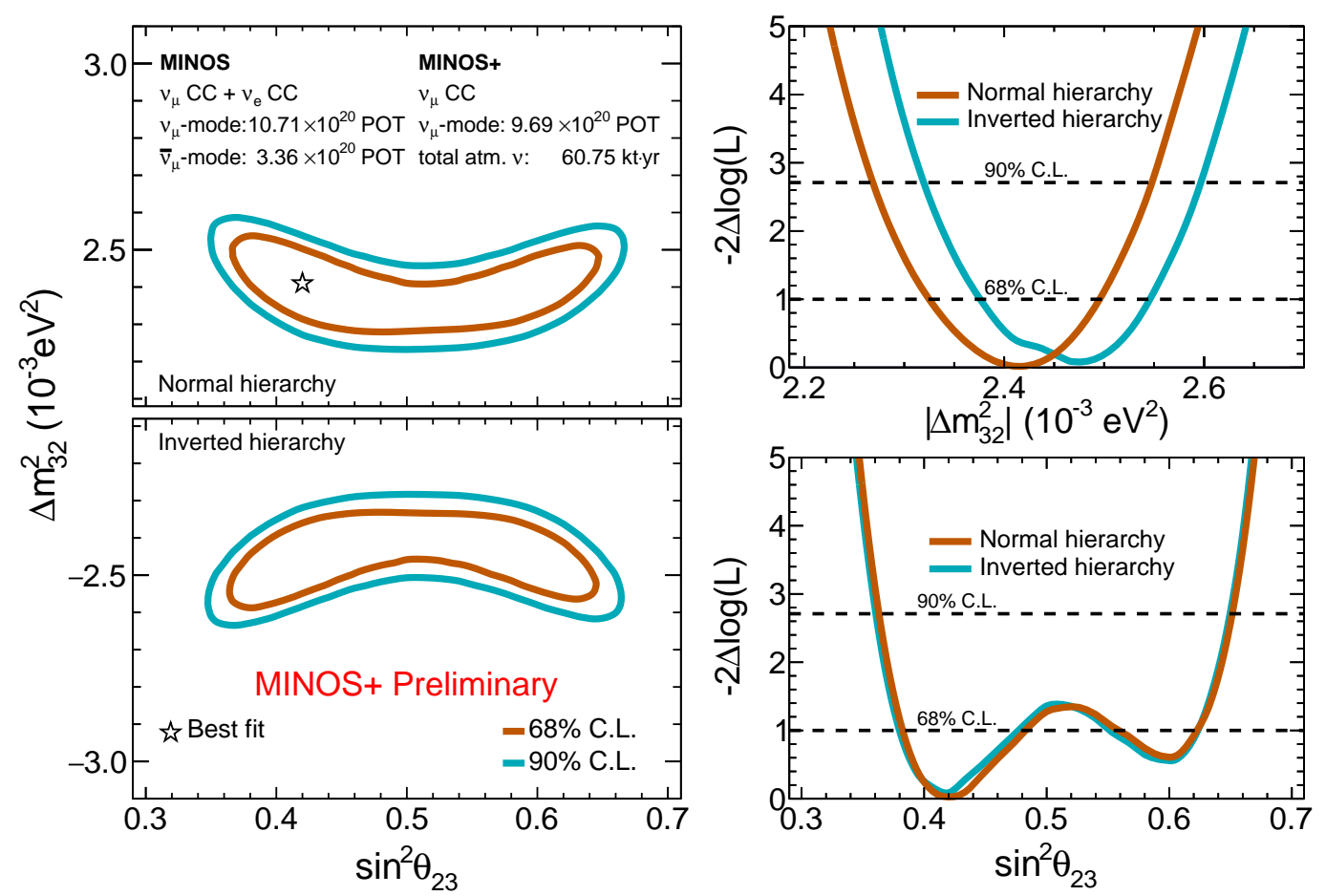

Figure 7.7: MINOS, MINOS+ combined $\Delta m_{32}^{2}$ and $\sin ^{2} \theta_{23}$ confidence limits. The left panel shows the $68 \%$ and $90 \%$ confidence limits on $\Delta m_{32}^{2}$ and $\sin ^{2} \theta_{23}$ for the normal mass hierarchy (top) and the inverted mass hierarchy (bottom). The best fit from the full MINOS+ beam exposure is plotted as a star. The right panels show the $1 \mathrm{D}$ likelihood profiles as functions of $\Delta m_{32}^{2}$ and $\sin ^{2} \theta_{23}$ for each mass hierarchy. 
Table 7.2: MINOS and MINOS+ combined best fit parameters for each mass hierarchy and $\theta_{23}$ octant. The value of $-2 \Delta \log (\mathcal{L})$ calculated relative to the overall best fit point is provided for each combination.

\begin{tabular}{ccccccc}
\hline Mass Hierarchy & $\theta_{23}$ & $\Delta m_{32}^{2} / 10^{-3} \mathrm{eV}^{2}$ & $\sin ^{2} \theta_{23}$ & $\sin ^{2} \theta_{13}$ & $\delta_{\mathrm{CP}} / \pi$ & $-2 \Delta \log (\mathcal{L})$ \\
\hline$\Delta m_{32}^{2}>0$ & $\theta_{23}>\pi / 4$ & 2.41 & 0.60 & 0.0209 & 0.69 & 0.60 \\
$\Delta m_{32}^{2}>0$ & $\theta_{23}<\pi / 4$ & 2.41 & 0.42 & 0.0210 & 0.50 & 0 \\
$\Delta m_{32}^{2}<0$ & $\theta_{23}>\pi / 4$ & -2.45 & 0.60 & 0.0210 & 0.45 & 0.52 \\
$\Delta m_{32}^{2}<0$ & $\theta_{23}<\pi / 4$ & -2.47 & 0.42 & 0.0211 & 2.0 & 0.08 \\
\hline
\end{tabular}

erences are detailed in Tables 7.3 to 7.5 .

The fit has a small preference for the normal mass hierarchy with $-2 \Delta \log (\mathcal{L})=0.08$. For the normal mass hierarchy the fit prefers the lower octant of $\theta_{23}$ by $-2 \Delta \log (\mathcal{L})=0.60$ and prefers non-maximal mixing at the 73.6\% confidence limit. For the inverted mass hierarchy, the fit prefers the lower octant of $\theta_{23}$ with $-2 \Delta \log (\mathcal{L})=0.48$ between the octant best fits. The fit also prefers non-maximal mixing at the $74.0 \%$ confidence limit in the inverted mass hierarchy.

\subsection{2 $\delta_{\mathrm{CP}}$ Constraints}

The $v_{\mathrm{e}}$-appearance data from MINOS in principle provides sensitivity to $\delta_{\mathrm{CP}}$. The modified data set used to calculate the $\delta_{\mathrm{CP}}$ limits in Fig. 7.8 includes the full MINOS beam and atmospheric exposure reported in Ref. [64] plus the full MINOS+ beam exposure. These data exclude $8 \%$ of the parameter space at $90 \%$ C.L. and $25 \%$ of the parameter space at $68 \%$ C.L. 
Table 7.3: MINOS, MINOS+ mass hierarchy confidence limits. $\left|\Delta m_{32}^{2}\right|$ is in units of $10^{-3} \mathrm{eV}^{2}$.

\begin{tabular}{cccc}
\hline Mass Hierarchy & Parameter & Best fit & Confidence limits \\
\hline \multirow{2}{*}{ Normal } & $\left|\Delta m_{32}^{2}\right|$ & 2.41 & $2.32-2.50(68 \%$ C.L. $)$ \\
& $\sin ^{2} \theta_{23}$ & 0.42 & $0.36-0.65(90 \%$ C.L.) \\
\hline \multirow{2}{*}{ Inverted } & $\left|\Delta m_{32}^{2}\right|$ & 2.47 & $2.37-2.55(68 \%$ C.L. $)$ \\
& $\sin ^{2} \theta_{23}$ & 0.42 & $0.36-0.65(90 \%$ C.L.) \\
\hline
\end{tabular}

Preference for normal mass hierarchy: $-2 \Delta \log (\mathcal{L})=0.08$

Table 7.4: MINOS, MINOS+ normal mass hierarchy octant confidence limits calculated from the 1D profiles with the fit preferences for lower octant and non-maximal mixing.

\begin{tabular}{cccc}
\hline Octant & Parameter & Best fit & Confidence limits \\
\hline Lower & $\sin ^{2} \theta_{23}$ & 0.42 & $0.38-0.48(68 \%$ C.L. $)$ \\
Upper & $\sin ^{2} \theta_{23}$ & 0.60 & $0.56-0.62(68 \%$ C.L. $)$ \\
\hline \multicolumn{4}{c}{ Preference for lower octant: $-2 \Delta \log (\mathcal{L})=0.60$} \\
Preference for non-maximal mixing: $-2 \Delta \log (\mathcal{L})=1.25(\Rightarrow 73.6 \%$ C.L. $)$
\end{tabular}

Table 7.5: MINOS, MINOS+ inverted mass hierarchy octant confidence limits calculated from the 1D profiles with the fit preferences for lower octant and non-maximal mixing.

\begin{tabular}{|c|c|c|c|}
\hline Octant & Parameter & Best fit & Confidence limits \\
\hline Lower & $\sin ^{2} \theta_{23}$ & 0.42 & $0.38-0.48$ (68\% C.L.) \\
\hline Upper & $\sin ^{2} \theta_{23}$ & 0.60 & $0.55-0.62$ (68\% C.L.) \\
\hline
\end{tabular}




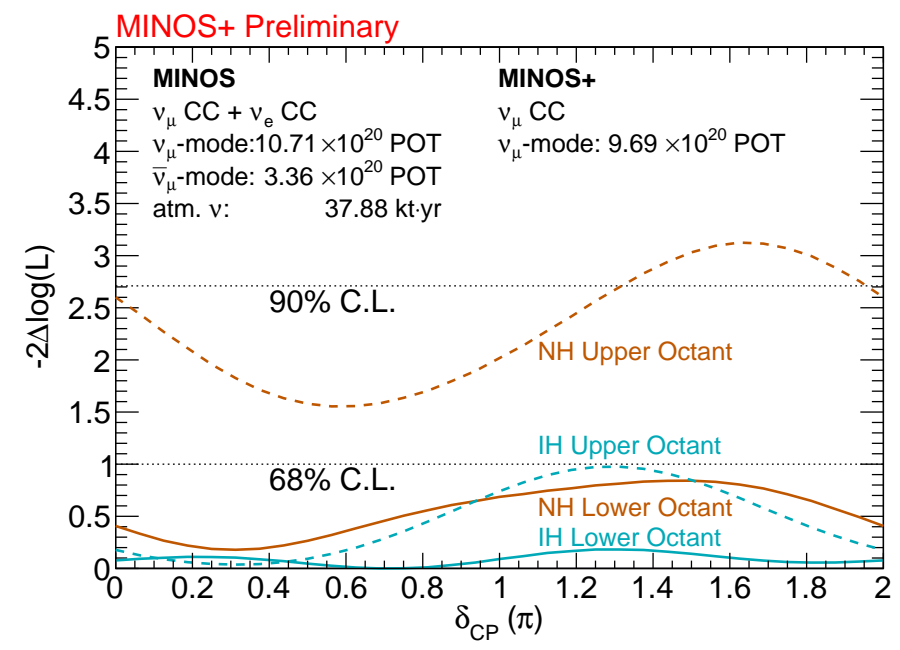

Figure 7.8: MINOS, MINOS+ combined $\delta_{\mathrm{CP}}$ profiles for each mass hierarchy and $\theta_{23}$ octant.

\subsubsection{Combining MINOS and MINOS+ with NOvA}

The NuMI neutrino beam offers the opportunity to simultaneously study the beam on-axis with MINOS and MINOS+ and off-axis with NOvA. As an initial attempt to combine data from all three experiments taking advantage of beam related cancellations, fit results from NOvA's muon neutrino disappearance sample are used to further constrain $\Delta m_{32}^{2}$ and $\sin ^{2} \theta_{23}$. Specifically, the samples from the experiments enable constraining the neutrino beam energy and expected flux at each detector, which impact the measurement of $\Delta m_{32}^{2}$. Since both experiments are measuring the same beam these uncertainties are reduced to the relative uncertainties between the experiments. The plan for the future is to do a combined fit of the data spectra properly correlating these systematic uncertainties between the data sets. The contours 
produced from this initial combination will serve as a figure of merit to compare with the future fit.

The NOvA fit used here was done to a sample of $\nu_{\mu}$-CC and $\bar{v}_{\mu}$-CC events from the NuMI beam in $v_{\mu}$-mode and $\bar{v}_{\mu}$-mode $[127,128]$. The $68 \%$ and $90 \%$ C.L. contours for the $\Delta m_{32}^{2}-\sin ^{2} \theta_{23}$ plane are reproduced in Fig. 7.9 in order to compare with the contours from the combination. This combination is performed by adding the NOvA $-2 \log (\mathcal{L})$ surface for $\Delta m_{32}^{2}$ and $\sin ^{2} \theta_{23}$ to the corresponding surface from the complete MINOS and MINOS+ data sets. In order to add the surfaces, the NOvA surface was calculated using the same 2D binning as the combined surface for MINOS and MINOS+. By simply adding the surfaces, this combination assumes the systematic uncertainties from NOvA are independent of MINOS and MINOS+.

The resulting $90 \%$ C.L. contours in Fig. 7.10 shrink in $\Delta m_{32}^{2}$ by $40 \%$ compared to the NOvA muon neutrino disappearance contours in Fig. 7.9. The contours span of $\sin ^{2} \theta_{23}$ remains virtually unchanged since the MINOS, MINOS+ data are predominately above the oscillation maximum, making the spectrum less sensitive to amplitude of disappearance which is determined by $\sin ^{2} \theta_{23}$ to leading order. 


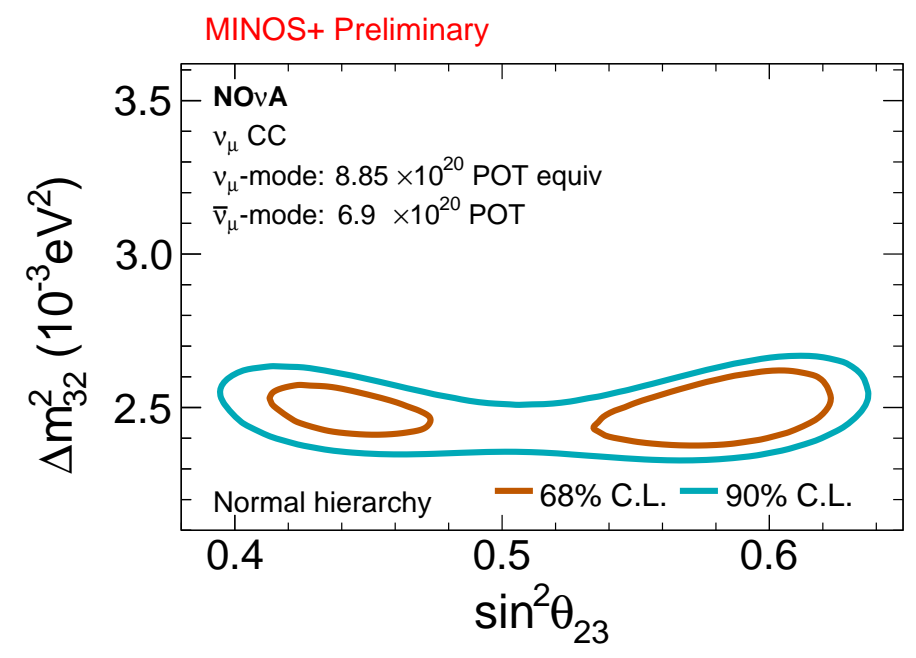

Figure 7.9: Reproduced $\Delta m_{32}^{2}$ and $\sin ^{2} \theta_{23}$ contours from NOvA's fit to muon neutrino disappearance $[127,128]$.

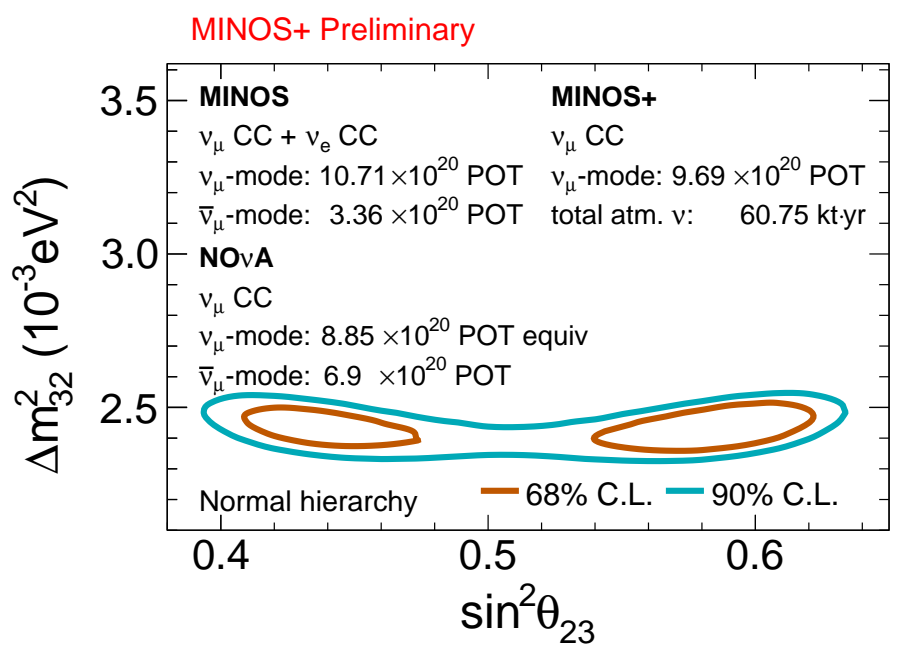

Figure 7.10: Initial $\Delta m_{32}^{2}$ and $\sin ^{2} \theta_{23}$ contours from combining MINOS, MINOS+, and NOvA fit surfaces. 


\section{Chapter 8}

\section{Summary}

This analysis of the MINOS+ complete beam data set finds no evidence for deviations from three-flavor oscillations with the limits on $\Delta m_{32}^{2}$ and $\sin ^{2} \theta_{23}$ containing the global best fit values within the $68 \%$ C.L. Together, MINOS and MINOS+ map the first muon neutrino disappearance maximum over a broad energy range, providing strong confirmation of three-flavor neutrino oscillations. Using the combined data set, the atmospheric oscillation parameters are measured as $\left|\Delta m_{32}^{2}\right|=[2.32-2.50] \times 10^{-3}(68 \%$ C.L. $)$ in the normal mass hierarchy and $\left|\Delta m_{32}^{2}\right|=[2.37-2.55] \times 10^{-3}(68 \%$ C.L. $)$ in the inverted mass hierarchy with $\sin ^{2} \theta_{23}=0.36-0.65$ (90\% C.L.) for both. The final combined measurement agrees with the results from other experiments in Fig. 8.1 and provides strong limits on $\Delta m_{32}^{2}$. In addition, this work updates the MINOS constraint of $\delta_{\mathrm{CP}}$, the $\theta_{23}$ octant, and mass hierarchy with the MINOS+ beam data set. This new constraint disfavors the normal mass hierarchy and upper $\theta_{23}$ octant at greater than $68 \%$ C.L.

This work performs the first combined constraint on $\Delta m_{32}^{2}$ and $\sin ^{2} \theta_{23}$ using data from the MINOS, MINOS+, and NOvA experiments, assuming the NOvA systematic uncertainties are independent. This combination is part 
of an effort to capitalize on the unique ability to measure the NuMI beam on-axis and off-axis to improve measurements of the atmospheric oscillation parameters. The combination improves the constraint on $\Delta m_{32}^{2}$ and motivates a combined fit using the experiments. Careful treatment of the systematic uncertainties related to the neutrino between the experiments remains to be done before conclusions can be drawn from such a combination.

Analysis of the MINOS+ data set has provided a new test of the threeflavor model and excluded large regions of parameter space for sterile neutrino scenarios $[129,130]$. MINOS+ continues to offer unique capabilities to measure three-flavor oscillations with the possibility to constrain beam related uncertainties in a novel joint fit with the NOvA experiment which should be pursued.

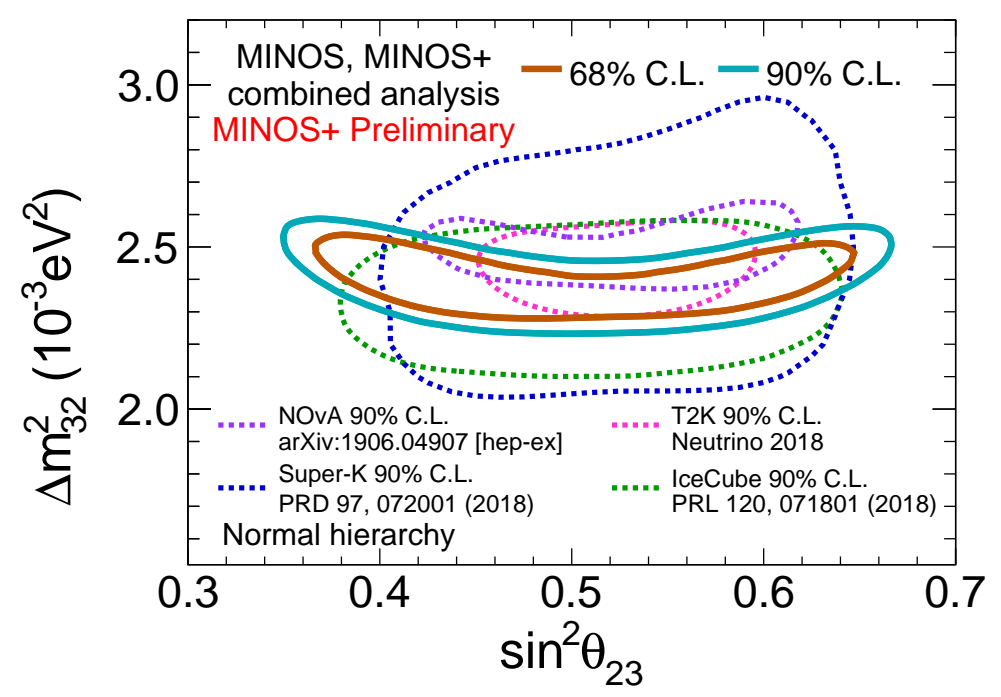

Figure 8.1: MINOS, MINOS $+\Delta m_{32}^{2}-\sin ^{2} \theta_{23}$ contours compared to other experiments [131-134]. 
Appendices 


\section{Appendix A}

\section{MINOS+ Run 12 and Run 13 Fit}

During MINOS+ running from 2014-2016, $4391 \boldsymbol{\nu}_{\mu}-\mathrm{CC}$ and $207 \overline{\boldsymbol{v}}_{\mu}-\mathrm{CC}$ events were measured at the FD. Taking into account systematic uncertainties, if neutrinos did not oscillate the predicted number of measured events at the FD would have been $5033 v_{\mu}$-CC and $209 \bar{v}_{\mu}$-CC. Figure A.1 shows the FD $v_{\mu}-\mathrm{CC}$ and $\bar{v}_{\mu}-\mathrm{CC}$ spectrum measured from 2014-2016 in black. The orange curve is the predicted spectrum with no oscillations and the best fit spectrum is shown in blue. The data and best fit are compared to the best fit measured using the full MINOS data set [64] shown in green.

Figure A.2 shows the ratios of data and fits to the unoscillated prediction for the MINOS+ data from 2014-2016. The ratio of data to the unoscillated prediction clearly shows muon neutrino disappearance.

Tables A.3 to A.6 show the fit preferences and limits for the MINOS+ data from 2014-2016. The last two years of MINOS+ data are consistent with the results from MINOS [64], and the confidence limits contain the limits from MINOS. With $69 \%$ of the MINOS+ beam exposure, the 2014-2016 runs

measure $\Delta m_{32}^{2}=2.35_{-3.0 \%}^{+8.5 \%} \times 10^{-3} \mathrm{eV}^{2}$ for the normal mass hierarchy and $\Delta m_{32}^{2}=2.41_{-5.4 \%}^{+7.9 \%} \times 10^{-3} \mathrm{eV}^{2}$ for the inverted mass hierarchy. 


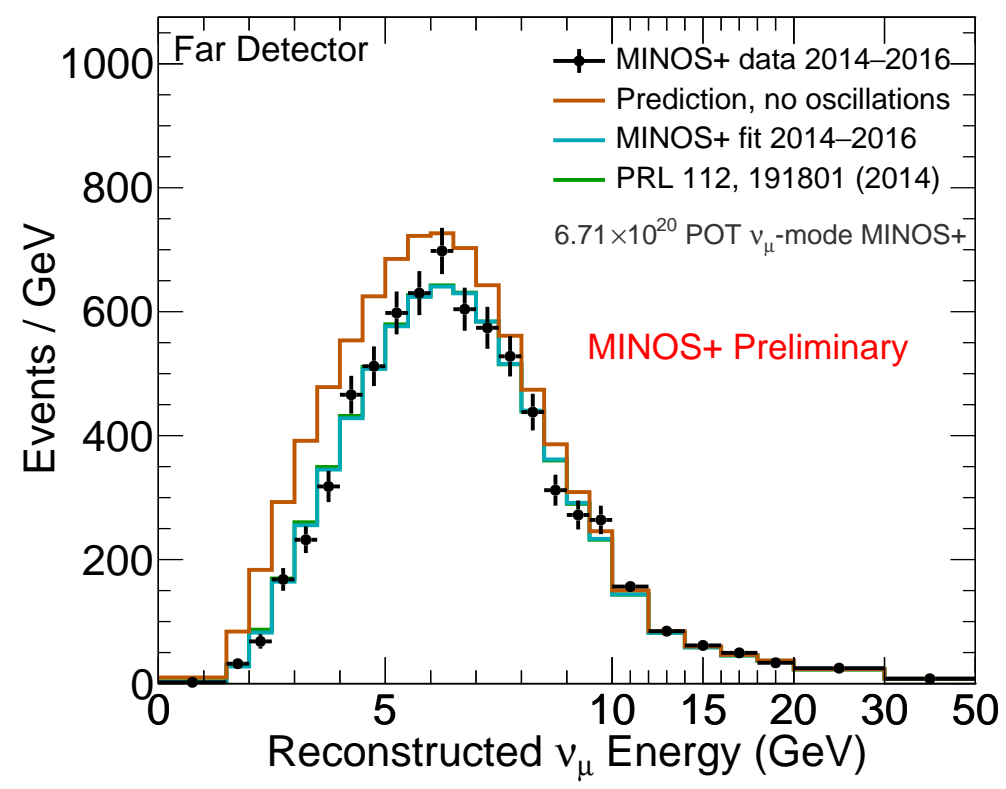

Figure A.1: Runs 12 and 13 combined contained-vertex $\nu_{\mu}$ and $\bar{v}_{\mu}$ data spectrum and predictions at the far detector. The data spectrum is shown as black points. The orange curve is the predicted spectrum with no oscillations, and the best fit spectrum is shown in blue. The predicted spectrum assuming the best fit measured using the full MINOS data set [64] is shown in green.

Table A.1 shows the breakdown of observed and predicted $\nu_{\mu}$ events for the last two years of MINOS+ beam exposure. The table shows the predicted number of events for no oscillations and the best fit, $\Delta m_{32}^{2}=2.35 \times 10^{-3} \mathrm{eV}^{2}$ and $\sin ^{2} \theta_{23}=0.52$. The best fit comes from a fit to the just the data from the last two years of MINOS+ beam exposure.

Table A.2 shows the breakdown of observed and predicted $\bar{v}_{\mu}$ events for the last two years of MINOS+ beam exposure. The table shows the predicted number of events for no oscillations and the best fit, $\Delta m_{32}^{2}=2.35 \times 10^{-3} \mathrm{eV}^{2}$ 


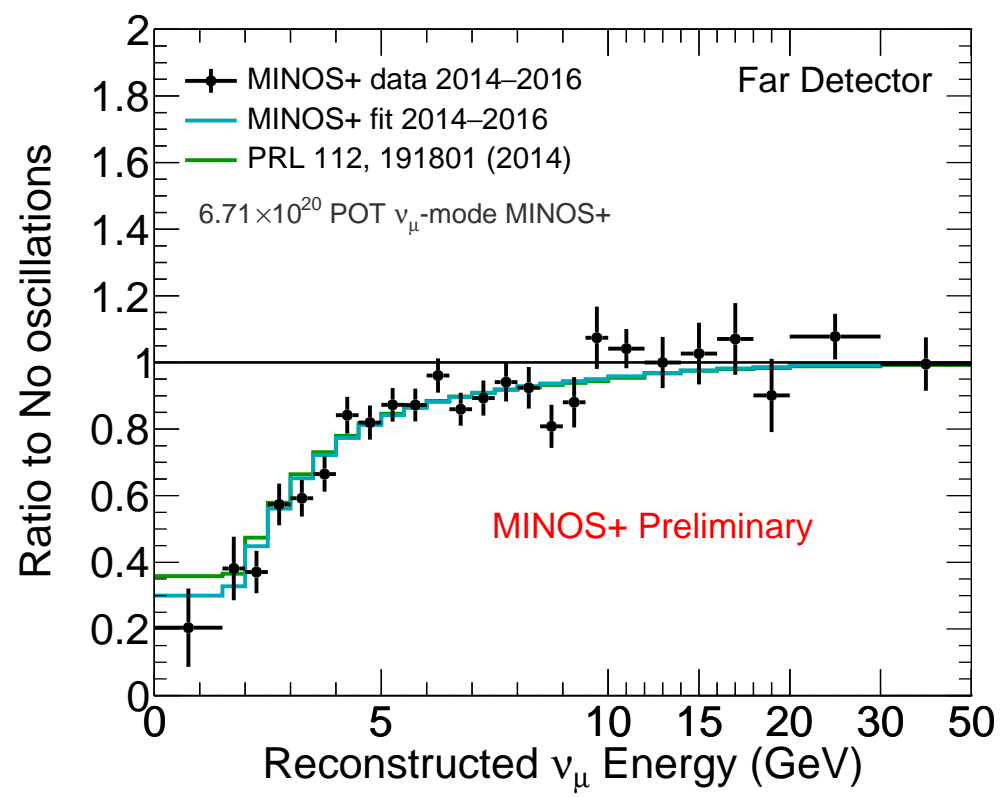

Figure A.2: Runs 12 and 13 combined ratios of data and best fit spectra to the no oscillations prediction. The data ratio in black clearly shows muon neutrino disappearance. The green curve is the ratio for the prediction assuming the MINOS best fit from Ref. [64]. The ratio for the best fit is drawn in blue.

and $\sin ^{2} \theta_{23}=0.52$. The best fit comes from a fit to the just the data from the last two years of MINOS+ beam exposure. Comparing the observed number of events in Tables A.1 and A.1 it is apparent the NuMI beam was operating in $v_{\mu}$-mode as the number of observed $v_{\mu}$ events is greater than the number of $\bar{v}_{\mu}$ events by more than an order of magnitude.

The fit preferences for each mass hierarchy and lower octant and upper octant of $\theta_{23}$ are shown in Table A.3. When each best fit point is compared to the global minimum in of $-2 \log (\mathcal{L})$ as is shown in the $-2 \Delta \log (\mathcal{L})$ column of Table A.3, the differences are very small. The table shows that these MINOS+ 
Table A.1: Runs 12 and 13 event counts of $\boldsymbol{v}_{\mu}$ from $\boldsymbol{v}_{\mu}$-mode beam.

\begin{tabular}{lrrr}
\hline Run & Observed & No Oscillations & Best Fit \\
\hline XII & 1803 & 2114 & 1821 \\
XIII & 2588 & 2919 & 2521 \\
\hline Total & 4391 & 5033 & 4342 \\
\hline
\end{tabular}

Table A.2: Runs 12 and 13 event counts of $\bar{v}_{\mu}$ from $v_{\mu}$-mode beam.

\begin{tabular}{lrrr}
\hline Run & Observed & No Oscillations & Best Fit \\
\hline XII & 93 & 88 & 78 \\
XIII & 114 & 121 & 108 \\
\hline Total & 207 & 209 & 186 \\
\hline
\end{tabular}

runs are not sensitive to the mass hierarchy or the octant of $\theta_{23}$. Note in Table A.3 that for $\Delta m_{32}^{2}>0$ and $\theta_{23}<\pi / 4$ the best fit is consistent with a value of $\theta_{23}$ in the lower octant; however, after rounding the value of $\sin ^{2} \theta_{23}=$ 0.50. It is expected that the MINOS+ data would not show a strong preference in $\sin ^{2} \theta_{23}$ since the peak of the neutrino beam spectrum is above the energy at which the muon neutrinos are most likely to oscillate.

Table A.4 directly compares the best fit point for that last two years of MINOS+ beam exposure in the normal mass hierarchy to the best fit point in the inverted mass hierarchy along with the $68 \%$ confidence limits for $\Delta m_{32}^{2}$ and $\sin ^{2} \theta_{23}$

Table A.5 compares normal mass hierarchy best fit points for $\sin ^{2} \theta_{23}$. 
Table A.3: MINOS+ Runs 12 and 13 combined best fit parameters for each mass hierarchy and $\theta_{23}$ octant. The value of $-2 \Delta \log (\mathcal{L})$ calculated relative to the overall best fit point is provided for each combination.

\begin{tabular}{ccccccc}
\hline Mass Hierarchy & $\theta_{23}$ & $\Delta m_{32}^{2} / 10^{-3} \mathrm{eV}^{2}$ & $\sin ^{2} \theta_{23}$ & $\sin ^{2} \theta_{13}$ & $\delta_{\mathrm{CP}} / \pi$ & $-2 \Delta \log (\mathcal{L})$ \\
\hline$\Delta m_{32}^{2}>0$ & $\theta_{23}>\pi / 4$ & 2.35 & 0.52 & 0.0210 & 1.79 & 0 \\
$\Delta m_{32}^{2}>0$ & $\theta_{23}<\pi / 4$ & 2.35 & 0.50 & 0.0210 & 1.86 & 0.006 \\
$\Delta m_{32}^{2}<0$ & $\theta_{23}>\pi / 4$ & -2.41 & 0.52 & 0.0210 & 2.0 & 0.008 \\
$\Delta m_{32}^{2}<0$ & $\theta_{23}<\pi / 4$ & -2.41 & 0.50 & 0.0210 & 1.83 & 0.01 \\
\hline
\end{tabular}

Table A.4: Runs 12 and 13 mass hierarchy confidence limits. $\left|\Delta m_{32}^{2}\right|$ is in units of $10^{-3} \mathrm{eV}^{2}$.

\begin{tabular}{cccc}
\hline Mass Hierarchy & Parameter & Best fit & Confidence limits \\
\hline \multirow{2}{*}{ Normal } & $\left|\Delta m_{32}^{2}\right|$ & 2.35 & $2.28-2.55(68 \%$ C.L. $)$ \\
& $\sin ^{2} \theta_{23}$ & 0.52 & $0.36-0.67(68 \%$ C.L. $)$ \\
\hline \multirow{2}{*}{ Inverted } & $\left|\Delta m_{32}^{2}\right|$ & 2.41 & $2.28-2.60$ (68\% C.L.) \\
& $\sin ^{2} \theta_{23}$ & 0.52 & $0.35-0.67$ (68\% C.L.) \\
\hline \multirow{2}{*}{ Prefer }
\end{tabular}

Preference for normal mass hierarchy: $-2 \Delta \log (\mathcal{L})=0.008$

The $68 \%$ confidence level contains $\sin ^{2} \theta_{23}=0.5$ and there is no strong preference for an octant.

The inverted mass hierarchy best fit points for $\sin ^{2} \theta_{23}$ are shown in Table A.6 with the $68 \%$ confidence levels. As with the normal mass ordering scenario there is no strong preference of an octant of $\theta_{23}$, and the $68 \%$ confidence level contains $\sin ^{2} \theta_{23}=0.5$.

Figure A.3 shows the 90\% confidence limits (blue) and 68\% confidence limits (orange) in the $\Delta m_{32}^{2}-\sin ^{2} \theta_{23}$ plane for the normal mass hierarchy 
Table A.5: Runs 12 and 13 normal mass hierarchy octant confidence limits calculated from the 1D profiles with the fit preferences for lower octant and non-maximal mixing.

\begin{tabular}{|c|c|c|c|}
\hline Octant & Parameter & Best fit & Confidence limits \\
\hline Lower & $\sin ^{2} \theta_{23}$ & 0.50 & $0.36-0.67$ (68\% C.L.) \\
\hline Upper & $\sin ^{2} \theta_{23}$ & 0.52 & 0.36-0.67 (68\% C.L.) \\
\hline
\end{tabular}

Table A.6: Runs 12 and 13 inverted mass hierarchy octant confidence limits calculated from the $1 \mathrm{D}$ profiles with the fit preferences for lower octant and non-maximal mixing.

\begin{tabular}{|c|c|c|c|}
\hline Octant & Parameter & Best fit & Confidence limits \\
\hline Lower & $\sin ^{2} \theta_{23}$ & 0.50 & $0.35-0.67$ (68\% C.L.) \\
\hline Upper & $\sin ^{2} \theta_{23}$ & 0.52 & $0.35-0.67$ (68\% C.L.) \\
\hline
\end{tabular}

$\left(\Delta m_{32}^{2}>0\right)$ and the inverted mass hierarchy $\left(\Delta m_{32}^{2}<0\right)$ for the last two years of MINOS+ beam data. The best fit is in the normal mass hierarchy and is shown with a black star.

Figure A.4 contains the contours as Fig. A.3 along with the confidence limits for $\left|\Delta m_{32}^{2}\right|$ and $\sin ^{2} \theta_{23}$. In the $1 \mathrm{D}$ limits, the normal mass hierarchy limit is in orange and the inverted mass hierarchy limit is in blue. For both 1D limits there is no visible preference for either mass hierarchy. Using this data set the confidence limits in $\sin ^{2} \theta_{23}$ are almost indistinguishable. 


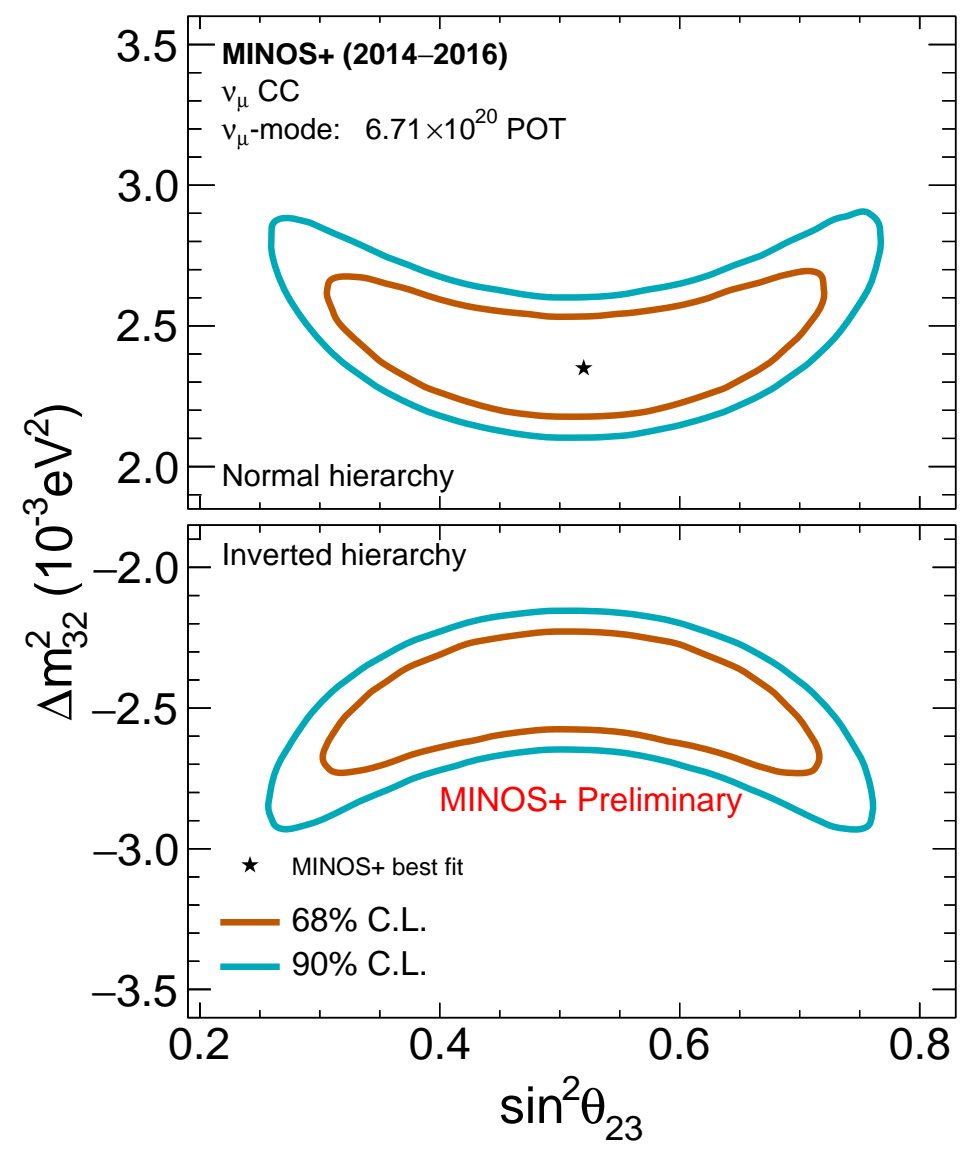

Figure A.3: Contours in $\Delta m_{32}^{2}$ and $\sin ^{2} \theta_{23}$ with $\Delta m_{32}^{2}$ from the fit to MINOS+ Runs 12 and 13 combined. The panel shows 68\% (orange) and 90\% (blue) confidence limits on $\Delta m_{32}^{2}$ and $\sin ^{2} \theta_{23}$ for the normal mass (top) and the inverted mass hierarchy (bottom). The best fit is plotted as a star. 

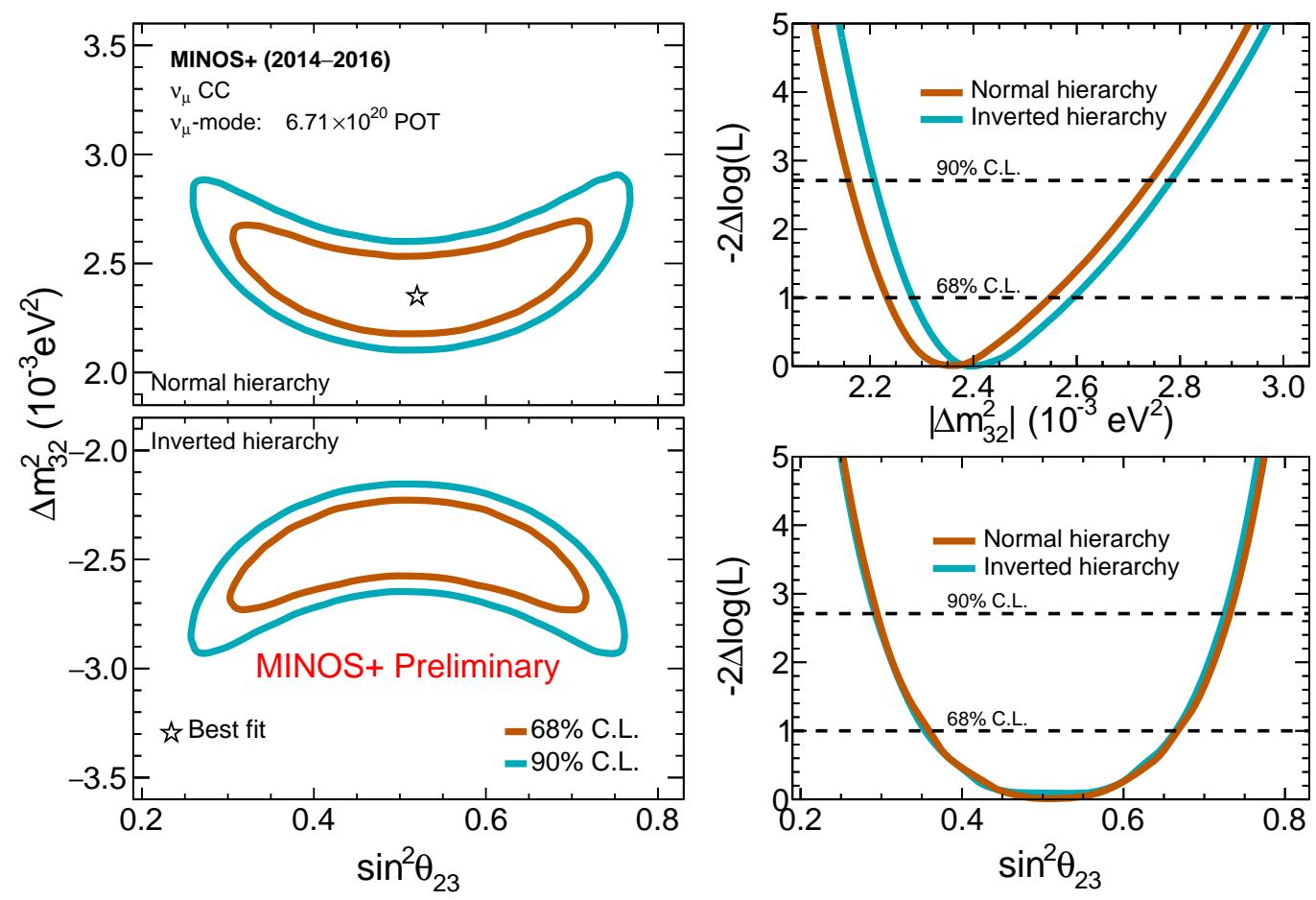

Figure A.4: MINOS+ Runs 12 and $13 \Delta m_{32}^{2}$ and $\sin ^{2} \theta_{23}$ confidence limits. The left panel shows the $68 \%$ and $90 \%$ confidence limits on $\Delta m_{32}^{2}$ and $\sin ^{2} \theta_{23}$ for the normal mass hierarchy (top) and the inverted mass hierarchy (bottom). The best fit from the full MINOS+ beam exposure is plotted as a star. The right panels show the 1D likelihood profiles as functions of $\Delta m_{32}^{2}$ and $\sin ^{2} \theta_{23}$ for each mass hierarchy. 


\section{Appendix B}

\section{MINOS+ Fit}

Table B.1: MINOS + event counts of $v_{\mu}$ from $v_{\mu}$-mode beam. The predicted number of events in the best fit column of the table come from fitting the measured $v_{\mu}$ and $\bar{v}_{\mu}$ events from the full MINOS+ data set.

\begin{tabular}{lrrr}
\hline Run & Observed & No Oscillations & Best Fit \\
\hline XI & 1889 & 2267 & 1939 \\
XII & 1803 & 2114 & 1808 \\
XIII & 2588 & 2919 & 2503 \\
\hline Total & 6280 & 7300 & 6250 \\
\hline
\end{tabular}

Table B.2: MINOS + event counts of $\bar{v}_{\mu}$ from $v_{\mu}$-mode beam. The predicted number of events in the best fit column of the table come from fitting the measured $v_{\mu}$ and $\bar{v}_{\mu}$ events from the full MINOS+ data set.

\begin{tabular}{lrrr}
\hline Run & Observed & No Oscillations & Best Fit \\
\hline XI & 86 & 93 & 83 \\
XII & 93 & 88 & 78 \\
XIII & 114 & 121 & 108 \\
\hline Total & 293 & 302 & 269 \\
\hline
\end{tabular}

Figure B.1 shows the $\Delta m_{32}^{2}-\sin ^{2} \theta_{23}$ confidence limits for the normal mass hierarchy and the inverted mass hierarchy using the full MINOS+ beam 
exposure. The best fit from the full MINOS+ beam exposure lies in the inverted mass hierarchy and upper octant of $\theta_{23}$.

Tables B.3 to B.6 show the fit preferences and limits for the MINOS+ data from 2013-2016. The MINOS+ data are consistent with the results from MINOS [64], and the confidence limits agree with the limits from MINOS. With the full MINOS+ exposure, MINOS+ measures $\Delta m_{32}^{2}=2.47_{-7.7 \%}^{+10.9 \%} \times 10^{-3} \mathrm{eV}^{2}$ for the normal mass hierarchy and $\Delta m_{32}^{2}=2.51_{-7.2 \%}^{+9.7 \%} \times 10^{-3} \mathrm{eV}^{2}$ for the inverted hierarchy.

The best fit points for each mass hierarchy and lower octant and upper octant of $\theta_{23}$ are shown in Table B.3. When each best fit point is compared to the global minimum in $-2 \log (\mathcal{L})$ there is no strong preference for hierarchy or octant from the MINOS+ data. The lack of preference is apparent from the 1D profiles in Fig. 7.3.

Table B.3: MINOS+ best fit parameters for each mass hierarchy and $\theta_{23}$ octant. The value of $-2 \Delta \log (\mathcal{L})$ calculated relative to the overall best fit point is provided for each combination.

\begin{tabular}{ccccccc}
\hline Mass Hierarchy & $\theta_{23}$ & $\Delta m_{32}^{2} / 10^{-3} \mathrm{eV}^{2}$ & $\sin ^{2} \theta_{23}$ & $\sin ^{2} \theta_{13}$ & $\delta_{\mathrm{CP}} / \pi$ & $-2 \Delta \log (\mathcal{L})$ \\
\hline$\Delta m_{32}^{2}>0$ & $\theta_{23}>\pi / 4$ & 2.47 & 0.63 & 0.0210 & 1.0 & 0.02 \\
$\Delta m_{32}^{2}>0$ & $\theta_{23}<\pi / 4$ & 2.47 & 0.40 & 0.0210 & 0.93 & 0.002 \\
$\Delta m_{32}^{2}<0$ & $\theta_{23}>\pi / 4$ & -2.51 & 0.62 & 0.0210 & 0.50 & 0 \\
$\Delta m_{32}^{2}<0$ & $\theta_{23}<\pi / 4$ & -2.52 & 0.40 & 0.0210 & 2.0 & 0.01 \\
\hline
\end{tabular}

The MINOS+ best fit points for each mass hierarchy are compared in Table B.4 with the corresponding $68 \%$ confidence limits. 


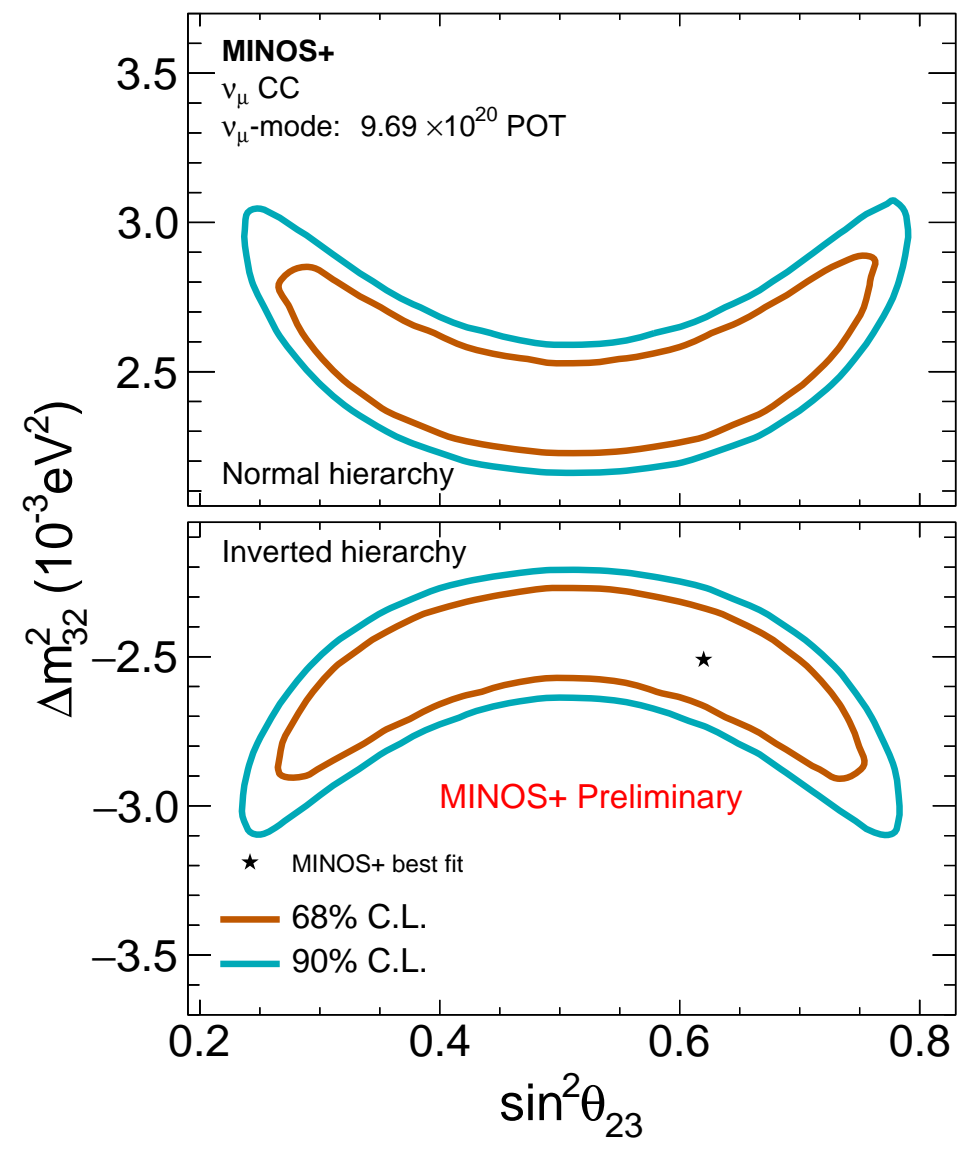

Figure B.1: MINOS+ contours in $\Delta m_{32}^{2}$ vs $\sin ^{2} \theta_{23}$. The $68 \%$ confidence limit is in orange, and the $90 \%$ confidence limit is in blue. The best fit from the full MINOS+ beam exposure, shown as the black star, lies in the inverted mass hierarchy and upper octant of $\theta_{23}$. 
Table B.4: MINOS+ mass hierarchy confidence limits. $\left|\Delta m_{32}^{2}\right|$ is in units of $10^{-3} \mathrm{eV}^{2}$.

\begin{tabular}{cccc}
\hline Mass Hierarchy & Parameter & Best fit & Confidence limits \\
\hline \multirow{2}{*}{ Normal } & $\left|\Delta m_{32}^{2}\right|$ & 2.47 & $2.28-2.74(68 \%$ C.L. $)$ \\
& $\sin ^{2} \theta_{23}$ & 0.40 & $0.30-0.73(68 \%$ C.L. $)$ \\
\hline \multirow{2}{*}{ Inverted } & $\left|\Delta m_{32}^{2}\right|$ & 2.51 & $2.33-2.78$ (68\% C.L.) \\
& $\sin ^{2} \theta_{23}$ & 0.62 & $0.30-0.72$ (68\% C.L.) \\
\hline
\end{tabular}

Preference for inverted mass hierarchy: $-2 \Delta \log (\mathcal{L})=0.002$

Table B.5: MINOS+ normal mass hierarchy octant confidence limits calculated from the 1D profiles with the fit preferences for lower octant and non-maximal mixing.

\begin{tabular}{cccc}
\hline Octant & Parameter & Best fit & Confidence limits \\
\hline Lower & $\sin ^{2} \theta_{23}$ & 0.40 & $0.30-0.73(68 \%$ C.L. $)$ \\
Upper & $\sin ^{2} \theta_{23}$ & 0.63 & $0.30-0.73(68 \%$ C.L. $)$ \\
\hline
\end{tabular}

Preference for lower octant: $-2 \Delta \log (\mathcal{L})=0.02$

Preference for non-maximal mixing: $-2 \Delta \log (\mathcal{L})=0.14(\Rightarrow 29.2 \%$ C.L. $)$

Table B.6: MINOS+ inverted mass hierarchy octant confidence limits calculated from the 1D profiles with the fit preferences for lower octant and non-maximal mixing.

\begin{tabular}{|c|c|c|c|}
\hline Octant & Parameter & Best fit & Confidence limits \\
\hline Lower & $\sin ^{2} \theta_{23}$ & 0.40 & $0.30-0.72$ (68\% C.L.) \\
\hline Upper & $\sin ^{2} \theta_{23}$ & 0.62 & 0.30-0.72 (68\% C.L.) \\
\hline
\end{tabular}




\section{Appendix C}

\section{MINOS and MINOS+ Fit}

Figure C.1 shows the total measured beam spectrum from neutrino events compared to the no oscillations prediction and best fit prediction using the entire MINOS and MINOS+ data set.

Table C.1 lists the total observed and predicted $v_{\mu}$ events detected in the fiducial volume of the FD from the total MINOS and MINOS $+v_{\mu}$-mode beams. The predicted number of events comes from the best fit to all the available MINOS and MINOS+ beam and atmospheric neutrino data including $v_{\mathrm{e}}$ appearance.

Table C.2 lists the detected and predicted $\bar{v}_{\mu}$ events in the fiducial volume of the FD from the $v_{\mu}$-mode beam.

Table C.3 list the detect and predicted number of $\bar{v}_{\mu}$ evens from the MINOS $\bar{v}_{\mu}$-mode beam. The predicted number of events comes from the best fit to the full MINOS and MINOS+ data set in Table 7.2.

Table C. 4 shows the detected muons at the FD in time with the MINOS $v_{\mu}$-mode beam compared to the predictions with no oscillations and the best fit for the whole MINOS and MINOS+ data set.

Table C.5 shows the detected and predicted atmospheric neutrino events 
Table C.1: MINOS and MINOS+ event counts of $v_{\mu}$ from $v_{\mu}$-mode beam. The predicted number of events in the best fit column of the table come from fitting the measured $v_{\mu}$ and $\bar{v}_{\mu}$ events from the full MINOS and MINOS+ data set.

\begin{tabular}{lrrr}
\hline Run & Observed & No Oscillations & Best Fit \\
\hline I & 292 & 390 & 296 \\
I HE & 119 & 134 & 125 \\
II & 457 & 585 & 446 \\
III & 900 & 1138 & 876 \\
V & 100 & 136 & 104 \\
VI & 138 & 183 & 140 \\
X & 573 & 709 & 540 \\
XI & 1889 & 2288 & 1962 \\
XII & 1803 & 2133 & 1829 \\
XIII & 2588 & 2945 & 2531 \\
\hline Total & 8859 & 10641 & 8849 \\
\hline
\end{tabular}

Table C.2: MINOS and MINOS+ event counts of $\bar{v}_{\mu}$ from $v_{\mu}$-mode beam. The predicted number of events in the best fit column of the table come from fitting the measured $v_{\mu}$ and $\bar{v}_{\mu}$ events from the full MINOS and MINOS+ data set.

\begin{tabular}{lrrr}
\hline Run & Observed & No Oscillations & Best Fit \\
\hline I & 24 & 44 & 38 \\
II & 51 & 68 & 59 \\
III & 134 & 138 & 121 \\
V & 13 & 16 & 14 \\
VI & 15 & 22 & 19 \\
X & 75 & 83 & 73 \\
XI & 86 & 94 & 84 \\
XII & 93 & 89 & 70 \\
XIII & 114 & 122 & 110 \\
\hline Total & 605 & 676 & 598 \\
\hline
\end{tabular}




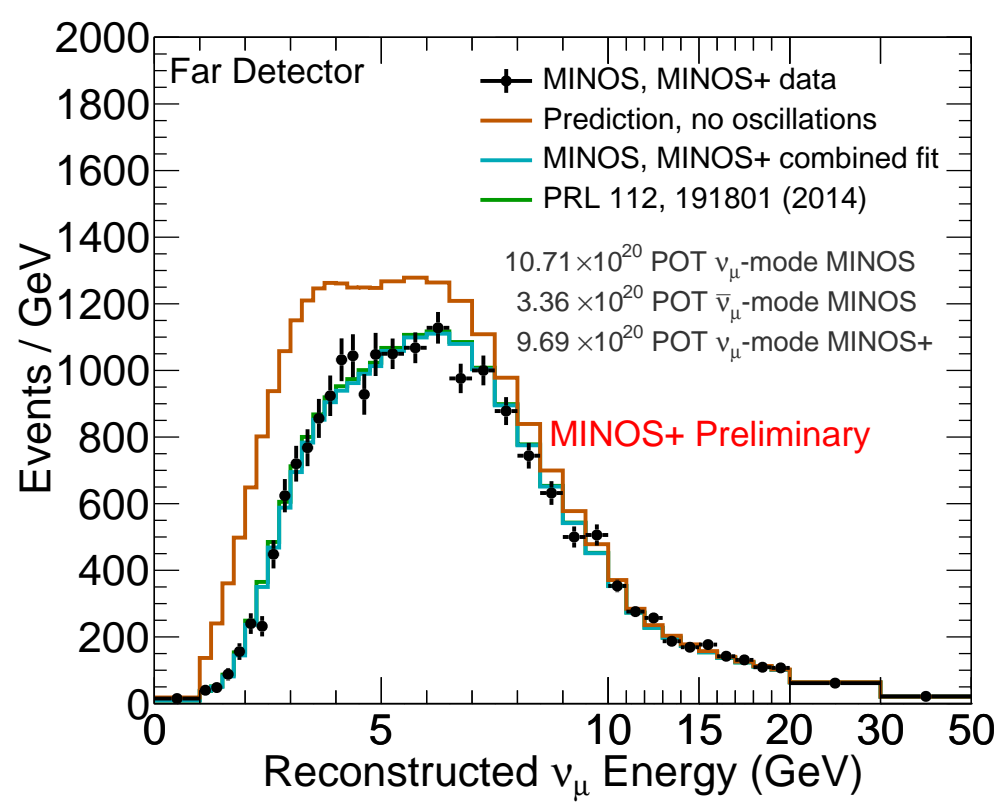

Figure C.1: MINOS and MINOS+ combined contained-vertex $v_{\mu}$ and $\bar{v}_{\mu}$ data spectrum and predictions at the far detector. The data spectrum is shown as black points. The orange curve is the predicted spectrum with no oscillations, and the blue curve is the best fit to the data for $\Delta m_{32}^{2}=2.41 \times 10^{-3} \mathrm{eV}^{2}$ and $\sin ^{2} \theta_{23}=0.42$. The predicted spectrum assuming the best fit measured using the full MINOS data set [64] is shown in green.

in the fiducial volume of the detector for the full $60.75 \mathrm{kt} \cdot \mathrm{yr}$. They $\nu_{\mu}$ or $\bar{v}_{\mu}$ category is for events with muon tracks that had ambiguous charge. The shower category is for $v_{\mathrm{e}}$-like interactions.

Table C.6 shows the number of observed muons as a result of an atmospheric neutrino interaction outside the fiducial volume.

Figure C.2 shows the $68 \%$ (orange) and $90 \%$ (blue) confidence limits for the full MINOS and MINOS+ data set. The best fit for the combined data 
set is shown in the normal mass hierarchy as a black star.

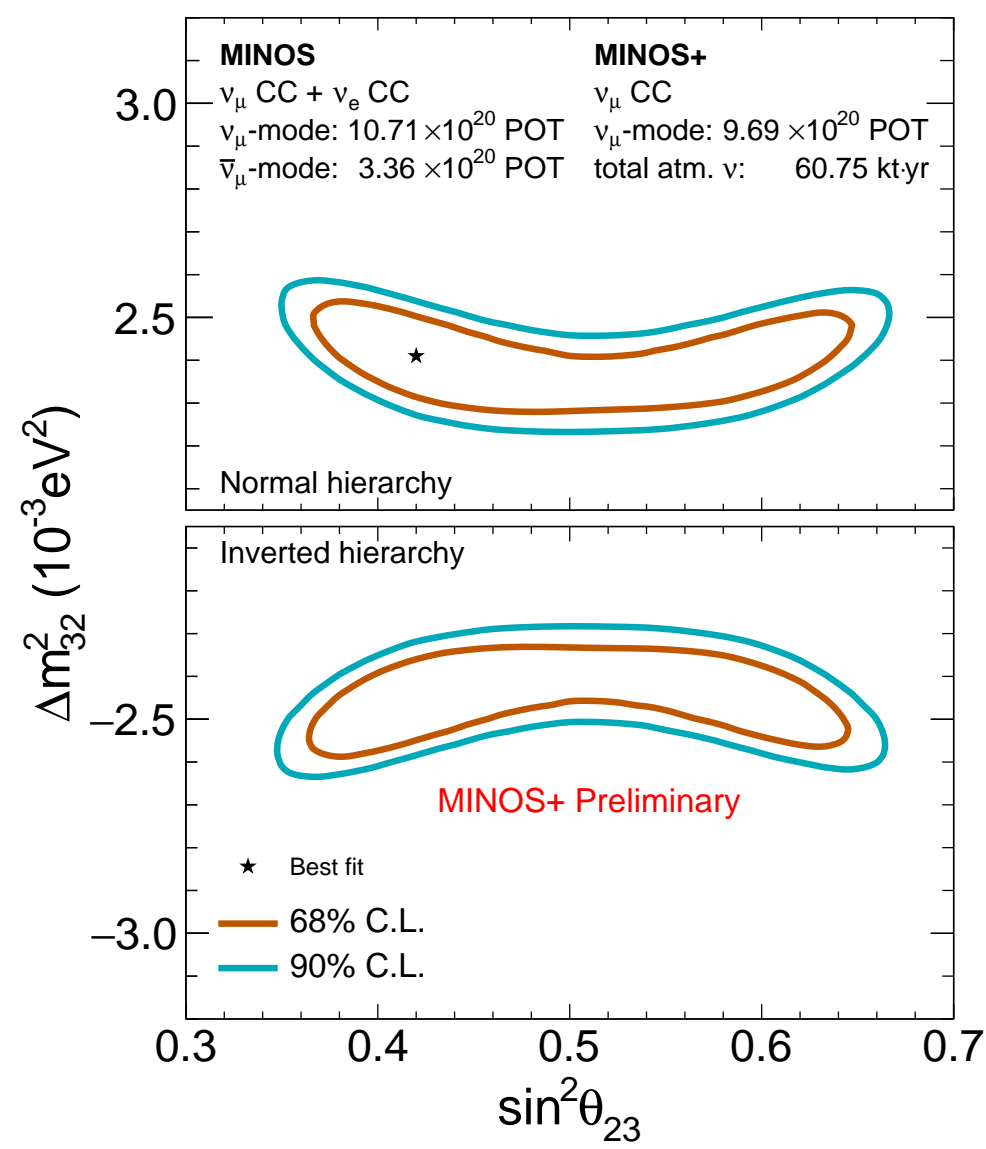

Figure C.2: MINOS and MINOS+ contours in $\Delta m_{32}^{2}$ and $\sin ^{2} \theta_{23}$. The $68 \%$ confidence limit is in orange, and the $90 \%$ confidence limit is in blue. The best fit from the full MINOS and MINOS+ neutrino exposure, shown as the black star, lies in the normal mass hierarchy and lower octant of $\theta_{23}$. 
Table C.3: MINOS event counts of $\bar{v}_{\mu}$ from $\bar{v}_{\mu}$-mode beam. The predicted number of events in the best fit column of the table come from fitting the measured $v_{\mu}$ and $\bar{v}_{\mu}$ events from the full MINOS and MINOS+ data set.

\begin{tabular}{lrrr}
\hline Run & Observed & No Oscillations & Best Fit \\
\hline IV & 99 & 164 & 115 \\
VII & 98 & 118 & 83 \\
IX & 29 & 38 & 27 \\
\hline Total & 226 & 320 & 225 \\
\hline
\end{tabular}

Table C.4: MINOS event counts of nonfiducial muons from $v_{\mu}$-mode beam. The predicted number of events in the best fit column of the table come from fitting the measured $v_{\mu}$ and $\bar{v}_{\mu}$ events from the full MINOS and MINOS+ data set.

\begin{tabular}{lrrr}
\hline Run & Observed & No Oscillations & Best Fit \\
\hline I & 357 & 382 & 330 \\
I HE & 128 & 135 & 128 \\
II & 555 & 575 & 497 \\
III & 977 & 1150 & 1003 \\
V & 116 & 134 & 117 \\
VI & 153 & 181 & 157 \\
X & 625 & 700 & 607 \\
\hline Total & 2911 & 3257 & 2839 \\
\hline
\end{tabular}


Table C.5: MINOS and MINOS+ event counts of CV atmospheric events. The predicted number of events in the best fit column of the table come from fitting the measured $v_{\mu}$ and $\bar{v}_{\mu}$ events from the full MINOS and MINOS+ data set.

\begin{tabular}{lrrr}
\hline Type & Observed & No Oscillations & Best Fit \\
\hline$v_{\mu}$ & 572 & 786 & 559 \\
$\bar{v}_{\mu}$ & 254 & 358 & 259 \\
$\boldsymbol{v}_{\mu}$ or $\bar{v}_{\mu}$ & 552 & 740 & 548 \\
shower & 1123 & 1223 & 1130 \\
\hline Total & 2501 & 3107 & 2496 \\
\hline
\end{tabular}

Table C.6: MINOS and MINOS+ event counts of nonfiducial neutrino induced muon (rock) atmospheric events. The predicted number of events in the best fit column of the table come from fitting the measured $\nu_{\mu}$ and $\bar{\nu}_{\mu}$ events from the full MINOS and MINOS+ data set.

\begin{tabular}{lrrr}
\hline Type & Observed & No Oscillations & Best Fit \\
\hline$v_{\mu}$ & 239 & 363 & 252 \\
$\bar{v}_{\mu}$ & 143 & 199 & 133 \\
$v_{\mu}$ or $\bar{v}_{\mu}$ & 354 & 371 & 350 \\
\hline Total & 736 & 933 & 735 \\
\hline
\end{tabular}




\section{Bibliography}

[1] C. L. Cowan, F. Reines, F. B. Harrison, H. W. Kruse, and A. D. McGuire, "Detection of the free neutrino: A Confirmation," Science, vol. 124, pp. 103-104, 1956.

[2] F. Reines, C. L. Cowan, F. B. Harrison, A. D. McGuire, and H. W. Kruse, "Detection of the free anti-neutrino," Phys. Rev., vol. 117, pp. 159173, 1960.

[3] G. Danby, J. M. Gaillard, K. A. Goulianos, L. M. Lederman, N. B. Mistry, M. Schwartz, and J. Steinberger, "Observation of High-Energy Neutrino Reactions and the Existence of Two Kinds of Neutrinos," Phys. Rev. Lett., vol. 9, pp. 36-44, 1962.

[4] K. Kodama et al., "Observation of tau neutrino interactions," Phys. Lett., vol. B504, pp. 218-224, 2001.

[5] S. Schael et al., "Precision electroweak measurements on the $Z$ resonance," Phys. Rept., vol. 427, pp. 257-454, 2006.

[6] P. F. de Salas and S. Pastor, "Relic neutrino decoupling with flavour oscillations revisited," JCAP, vol. 1607, no. 07, p. 051, 2016.

[7] N. Aghanim et al., "Planck 2018 results. VI. Cosmological parameters," 2018. 
[8] J. Ellis, "TikZ-Feynman: Feynman diagrams with TikZ," Comput. Phys. Commun., vol. 210, pp. 103-123, 2017.

[9] R. Davis, Jr., D. S. Harmer, and K. C. Hoffman, "Search for neutrinos from the sun," Phys. Rev. Lett., vol. 20, pp. 1205-1209, 1968.

[10] J. N. Bahcall, N. A. Bahcall, and G. Shaviv, "Present status of the theoretical predictions for the $\mathrm{Cl}-36$ solar neutrino experiment," Phys. Rev. Lett., vol. 20, pp. 1209-1212, 1968. [,45(1968)].

[11] Q. R. Ahmad et al., "Measurement of the rate of $\nu_{e}+d \rightarrow p+p+e^{-}$ interactions produced by ${ }^{8} B$ solar neutrinos at the Sudbury Neutrino Observatory," Phys. Rev. Lett., vol. 87, p. 071301, 2001.

[12] Q. R. Ahmad et al., "Direct evidence for neutrino flavor transformation from neutral current interactions in the Sudbury Neutrino Observatory," Phys. Rev. Lett., vol. 89, p. 011301, 2002.

[13] K. S. Hirata et al., "Experimental Study of the Atmospheric Neutrino Flux," Phys. Lett., vol. B205, p. 416, 1988. [,447(1988)].

[14] T. Kajita, "Atmospheric neutrino results from Super-Kamiokande and Kamiokande: Evidence for neutrino(mu) oscillations," Nucl. Phys. Proc. Suppl., vol. 77, pp. 123-132, 1999. [,123(1998)].

[15] Y. Fukuda et al., "Evidence for oscillation of atmospheric neutrinos," Phys. Rev. Lett., vol. 81, pp. 1562-1567, 1998. 
[16] B. Pontecorvo, "Mesonium and anti-mesonium," Sov. Phys. JETP, vol. 6, p. 429, 1957. [Zh. Eksp. Teor. Fiz.33,549(1957)].

[17] B. Pontecorvo, "Inverse beta processes and nonconservation of lepton charge," Sov. Phys. JETP, vol. 7, pp. 172-173, 1958. [Zh. Eksp. Teor. Fiz.34,247(1957)].

[18] Z. Maki, M. Nakagawa, and S. Sakata, "Remarks on the unified model of elementary particles," Prog. Theor. Phys., vol. 28, pp. 870-880, 1962. $[, 34(1962)]$.

[19] C. Giunti and C. W. Kim, Fundamentals of Neutrino Physics and Astrophysics. 2007.

[20] S. P. Mikheyev and A. Yu. Smirnov, "Resonance Amplification of Oscillations in Matter and Spectroscopy of Solar Neutrinos," Sov. J. Nucl. Phys., vol. 42, pp. 913-917, 1985. [,305(1986)].

[21] S. P. Mikheev and A. Yu. Smirnov, "Resonant amplification of neutrino oscillations in matter and solar neutrino spectroscopy," Nuovo Cim., vol. C9, pp. 17-26, 1986.

[22] L. Wolfenstein, "Neutrino Oscillations in Matter," Phys. Rev., vol. D17, pp. 2369-2374, 1978. [,294(1977)].

[23] D. Adey et al., "Measurement of the Electron Antineutrino Oscillation with 1958 Days of Operation at Daya Bay," Phys. Rev. Lett., vol. 121, no. 24, p. $241805,2018$. 
[24] G. Bak et al., "Measurement of Reactor Antineutrino Oscillation Amplitude and Frequency at RENO," Phys. Rev. Lett., vol. 121, no. 20, p. 201801, 2018.

[25] Y. Abe et al., "Measurement of $\theta_{13}$ in Double Chooz using neutron captures on hydrogen with novel background rejection techniques," JHEP, vol. 01, p. 163, 2016.

[26] M. Tanabashi et al., "Review of Particle Physics," Phys. Rev., vol. D98, no. 3, p. 030001, 2018.

[27] G. Altarelli and F. Feruglio, "Discrete Flavor Symmetries and Models of Neutrino Mixing," Rev. Mod. Phys., vol. 82, pp. 2701-2729, 2010.

[28] S. F. King, A. Merle, S. Morisi, Y. Shimizu, and M. Tanimoto, "Neutrino Mass and Mixing: from Theory to Experiment," New J. Phys., vol. 16, p. $045018,2014$.

[29] I. Girardi, S. T. Petcov, A. J. Stuart, and A. V. Titov, "Leptonic Dirac CP Violation Predictions from Residual Discrete Symmetries," Nucl. Phys., vol. B902, pp. 1-57, 2016.

[30] S. Pascoli, S. T. Petcov, and A. Riotto, "Connecting low energy leptonic CP-violation to leptogenesis," Phys. Rev., vol. D75, p. 083511, 2007.

[31] E. Molinaro and S. T. Petcov, "A Case of Subdominant/Suppressed 'High Energy' Contribution to the Baryon Asymmetry of the Universe in Flavoured Leptogenesis," Phys. Lett., vol. B671, pp. 60-65, 2009. 
[32] S. Pascoli, S. T. Petcov, and A. Riotto, "Leptogenesis and Low Energy CP Violation in Neutrino Physics," Nucl. Phys., vol. B774, pp. 1-52, 2007.

[33] S. E. Kopp, "Accelerator-based neutrino beams," Phys. Rept., vol. 439, pp. 101-159, 2007.

[34] G. Tzanankos et al., "MINOS+: a Proposal to FNAL to run MINOS with the medium energy NuMI beam," 2011.

[35] D. S. Ayres et al., "The NOvA Technical Design Report," 2007.

[36] P. Adamson et al., "Search for Sterile Neutrinos Mixing with Muon Neutrinos in MINOS," Phys. Rev. Lett., vol. 117, no. 15, p. 151803, 2016.

[37] P. Adamson et al., "Constraints on Large Extra Dimensions from the MINOS Experiment," Phys. Rev., vol. D94, no. 11, p. 111101, 2016.

[38] P. Adamson et al., "Search for flavor-changing nonstandard neutrino interactions using $\nu_{e}$ appearance in MINOS," Phys. Rev., vol. D95, no. 1, p. 012005, 2017.

[39] "The Fermilab Main Injector Technical Design Handbook," FERMILABDESIGN 1994-01, 1994.

[40] P. Adamson et al., "A Study of Muon Neutrino Disappearance Using the Fermilab Main Injector Neutrino Beam," Phys. Rev., vol. D77, p. $072002,2008$. 
[41] Z. Pavlovic, Observation of Disappearance of Muon Neutrinos in the NuMI Beam. PhD thesis, Texas U., 2008.

[42] P. Adamson et al., "The NuMI Neutrino Beam," Nucl. Instrum. Meth., vol. A806, pp. 279-306, 2016.

[43] W. Pellico, K. Domann, F. Garcia, K. Gollwitzer, K. Seiya, and R. Zwaska, "FNAL - The Proton Improvement Plan (PIP)," in Proceedings, 5th International Particle Accelerator Conference (IPAC 2014): Dresden, Germany, June 15-20, 2014, p. THPME075, 2014.

[44] P. Adamson, "Reuse Recycler: High Intensity Proton Stacking at Fermilab," in Proceedings, 57th ICFA Advanced Beam Dynamics Workshop on High-Intensity and High-Brightness Hadron Beams (HB2016): Malm, Sweden, July 3-8, 2016, p. THAM1X01, 2016.

[45] R. M. Zwaska, Accelerator Systems and Instrumentation for the NuMI Neutrino Beam. PhD thesis, Texas U., 2005.

[46] R. Zwaska et al., "Beam-Based Alignment of the NuMI Target Station Components at FNAL," Nucl. Instrum. Meth., vol. A568, pp. 548-560, 2006.

[47] M. Kostin, S. Kopp, M. Messier, D. A. Harris, J. Hylen, and A. Para, "Proposal for continuously-variable neutrino beam energy for the NuMI facility," 2006. 
[48] K. Ammigan, "NuMI-NOvA Target \& Window," 10th International Workshop on Neutrino Beams and Instrumentation, Sep 2017.

[49] A. Holin, A. Radovic, and A. Schreckenberger, "Blessed plots for the 2014 beam systematics group," MINOS Doc 10630, May 2014.

[50] D. G. Michael et al., "The Magnetized steel and scintillator calorimeters of the MINOS experiment," Nucl. Instrum. Meth., vol. A596, pp. 190$228,2008$.

[51] K. Lang et al., "A comprehensive characterization of Hamamatsu 16and 64-anode PMTs," Nucl. Instrum. Meth., vol. A461, pp. 571-573, 2001.

[52] K. Lang et al., "Characterization of 1600 Hamamatsu 16-anode photomultipliers for the MINOS Far detector," Nucl. Instrum. Meth., vol. A545, pp. 852-871, 2005.

[53] The MINOS Collaboration, "The minos detectors technical design report," Fermilab report NuMI-L-337, Oct 1998.

[54] N. Tagg, A. De Santo, A. Weber, A. Cabrera, P. S. Miyagawa, M. A. Barker, K. Lang, D. Michael, R. Saakyan, and J. Thomas, "Performance of Hamamatsu 64-anode photomultipliers for use with wavelength-shifting optical fibres," Nucl. Instrum. Meth., vol. A539, pp. 668-678, 2005.

[55] A. Blake, "Atmospheric neutrino status," MINOS Doc 12792, Feb 2018. 
[56] A. Blake, "Atmospheric neutrino results and plots (using complete 60.75 kt-yr data set)," MINOS Doc 13110, May 2018.

[57] J. J. Evans, Measuring Antineutrino Oscillations with the MINOS Experiment. PhD thesis, Oxford U., 2008.

[58] P. Adamson et al., "Electron neutrino and antineutrino appearance in the full MINOS data sample," Phys. Rev. Lett., vol. 110, no. 17, p. 171801, 2013.

[59] J. S. Marshall, A study of muon neutrino disappearance with the MINOS detectors and the NuMI neutrino beam. PhD thesis, Cambridge U., 2008.

[60] D. E. Groom, N. V. Mokhov, and S. I. Striganov, "Muon stopping power and range tables $10-\mathrm{MeV}$ to $100-\mathrm{TeV}, "$ Atom. Data Nucl. Data Tabl., vol. 78, pp. 183-356, 2001.

[61] T. M. Cover and P. E. Hart, "Nearest neighbor pattern classification," IEEE Trans. Inf. Theory, vol. 13, p. 21, 1967.

[62] C. J. Backhouse, Measuring neutrino oscillation parameters using $\nu_{\mu}$ disappearance in MINOS. PhD thesis, Oxford U., 2011.

[63] J. Huang, Sterile Neutrino Searches in MINOS/MINOS+ Experiment. PhD thesis, U. Texas, Austin (main), 2015.

[64] P. Adamson et al., "Combined analysis of $\nu_{\mu}$ disappearance and $\nu_{\mu} \rightarrow \nu_{e}$ appearance in MINOS using accelerator and atmospheric neutrinos," Phys. Rev. Lett., vol. 112, p. 191801, 2014. 
[65] A. Blake, "Far detector pot integration for minos physics analyses," MINOS Doc 9093, May 2012.

[66] A. Blake, "Data validation for run 13," MINOS Doc 11640, Feb 2017.

[67] P. Adamson et al., "Measurements of atmospheric neutrinos and antineutrinos in the minos far detector," Phys. Rev. D, vol. 86, p. 052007, Sep 2012.

[68] A. J. Perch, Three-flavour neutrino oscillations with MINOS and CHIPS. PhD thesis, University Coll. London, 2017-01-17.

[69] P. Adamson et al., "Measurement of Neutrino and Antineutrino Oscillations Using Beam and Atmospheric Data in MINOS," Phys. Rev. Lett., vol. 110, no. 25, p. 251801, 2013.

[70] R. Ospanov, A measurement of muon neutrino disappearance with the MINOS detectors and NuMI beam. PhD thesis, Texas U., 2008.

[71] A. Radovic, Measuring the Disappearance of Muon Neutrinos with the MINOS Detector. PhD thesis, U. Coll. London, 2013.

[72] J. S. Ratchford, Identifying Muons for Neutrino Oscillation and Cross Section Experiments. PhD thesis, Texas U., ARL, 2012.

[73] L. Whitehead, "Nd coil hole studies," MINOS Doc 10643, May 2014. 
[74] M. L. Strait, Measurement of Neutrino Oscillation Parameters Using Anti-fiducial Charged Current Events in MINOS. PhD thesis, Minnesota U., 2010.

[75] J. P. Ochoa Ricoux, A search for muon neutrino to electron neutrino oscillations in the MINOS Experiment. PhD thesis, Caltech, 2009.

[76] R. B. Toner, Measuring $\theta_{13}$ via Muon Neutrino to Electron Neutrino Oscillations in the MINOS Experiment. $\mathrm{PhD}$ thesis, Cambridge U., 2011.

[77] M. Campanella, A. Ferrari, P. R. Sala, and S. Vanini, "Reusing Code from FLUKA and GEANT4 Geometry," 1998.

[78] M. Campanella, A. Ferrari, P. R. Sala, and S. Vanini, "First Calorimeter Simulation with the FLUGG Prototype," 1999.

[79] T. T. Böhlen, F. Cerutti, M. P. W. Chin, A. Fassò, A. Ferrari, P. G. Ortega, A. Mairani, P. R. Sala, G. Smirnov, and V. Vlachoudis, "The FLUKA Code: Developments and Challenges for High Energy and Medical Applications," Nucl. Data Sheets, vol. 120, pp. 211-214, 2014.

[80] A. Ferrari, P. R. Sala, A. Fasso, and J. Ranft, "FLUKA: A multi-particle transport code (Program version 2005)," 2005.

[81] S. Agostinelli et al., "GEANT4: A Simulation toolkit," Nucl. Instrum. Meth., vol. A506, pp. 250-303, 2003. 
[82] J. Allison et al., "Geant4 developments and applications," IEEE Trans. Nucl. Sci., vol. 53, p. 270, 2006.

[83] J. Allison et al., "Recent developments in Geant4," Nucl. Instrum. Meth., vol. A835, pp. 186-225, 2016.

[84] A. Holin, "Minos lessons for the numi beam flux," June 2018.

[85] A. V. Lebedev, Ratio of pion kaon production in proton carbon interactions. PhD thesis, Harvard U., 2007.

[86] J. Biggs, K. Bourkland, J. Hylen, and M. Kucera, "Calibration of numi horn current readout," MINOS Doc 1303, Aug 2006.

[87] J. Hylen and K. Yonehara, "2018 re-calibration of numi horn current readout," MINOS Doc 13369, Nov 2018.

[88] J. Hylen, "Intro to horn water spray," NuMI-X Doc 93, Nov 2014.

[89] A. Holin, I. Anghel, and A. Schreckenberger, "The minos+ beam fit and beam systematic error bands," MINOS Doc 10946, Feb 2015.

[90] J. Hylen, "Systematic uncertainties in the numi beam flux," MINOS Doc 1283, May 2007.

[91] A. Holin, I. Anghel, M. Pfutzner, A. Schreckenberger, and P. Vahle, "Beam fitting and flux systematic errors position paper," MINOS Doc 10577, Apr 2014. 
[92] A. Schreckenberger, Apr 2019. private communication.

[93] J. Hylen, "Numi horn 1 mis-alignment," MINOS Doc 11699, Nov 2016.

[94] J. Hylen, "Numi horn 1 mis-alignment," NuMI-X Doc 131, Nov 2016.

[95] T. Carroll, "Summary of horn tilt simulations," MINOS Doc 11754, Feb 2017.

[96] T. Carroll, "Summary of horn tilt simulations," NuMI-X Doc 151, Feb 2017.

[97] G. Brunetti, "Muon monitors in 2015/2016 run," NuMI-X Doc 135, Nov 2016.

[98] W. Flanagan, "Horn tilt summary," MINOS Doc 11740-v4, Feb 2017.

[99] A. Holin and J. Thomas, "Beam fits for standard oscillations analysis," MINOS Doc 13061, May 2018.

[100] M. Szleper and A. Para, "Neutrino spectrum at the far detector systematic errors," 2001.

[101] A. I. Himmel, Antineutrino Oscillations in the Atmospheric Sector. PhD thesis, Caltech, 2011.

[102] J. Kopp, "Efficient numerical diagonalization of hermitian 3 x 3 matrices," Int. J. Mod. Phys., vol. C19, pp. 523-548, 2008. 
[103] A. Radovic and J. Coelho, "Position paper describing the derivation of the matter density in the numi beam," MINOS Doc 9765, Apr 2013.

[104] G. D. Barr, T. K. Gaisser, P. Lipari, S. Robbins, and T. Stanev, "A Three - dimensional calculation of atmospheric neutrinos," Phys. Rev., vol. D70, p. 023006, 2004.

[105] A. M. Dziewonski and D. L. Anderson, "Preliminary reference earth model," Phys. Earth Planet. Interiors, vol. 25, pp. 297-356, 1981.

[106] F. James and M. Roos, "Minuit: A System for Function Minimization and Analysis of the Parameter Errors and Correlations," Comput. Phys. Commun., vol. 10, pp. 343-367, 1975.

[107] S. Baker and R. D. Cousins, "Clarification of the Use of Chi Square and Likelihood Functions in Fits to Histograms," Nucl. Instrum. Meth., vol. 221, pp. 437-442, 1984.

[108] G. L. Fogli, E. Lisi, A. Marrone, D. Montanino, A. Palazzo, and A. M. Rotunno, "Global analysis of neutrino masses, mixings and phases: entering the era of leptonic CP violation searches," Phys. Rev., vol. D86, p. 013012, 2012.

[109] C. Patrignani et al., "Review of Particle Physics," Chin. Phys., vol. C40, no. 10, p. 100001, 2016.

[110] R. E. Armstrong, Muon neutrino disappearance at MINOS. PhD thesis, Indiana U., 2009. 
[111] J. M. Paley, "Hand-scan estimation of the reconstruction-related systematic uncertainty in the near/far normalization of the minos cc analysis," MINOS Doc 5613, Indiana U., Jan 2009.

[112] P. A. Rodrigues, "Notes on the normalization systematic," MINOS Doc 6636, Mar 2010.

[113] J. Marshall, "A data-driven correction to the mc nc background," MINOS Doc 3307, Jun 2007.

[114] L. Whitehead, "Normalisation and nc background systematics for cc2014," MINOS Doc 10510, Apr 2014.

[115] M. Kordosky, H. R. Gallagher, and S. Dytman, "Shower energy scale uncertainty for the run i+ii cc analysis," MINOS Doc 4287, Mar 2008.

[116] MINOS Calibration Group, "2009 calibraiton position paper on runs i-ii-iii," MINOS Doc 6717, Jan 2010.

[117] R. Hatcher et al., "Range curvature task force position paper," MINOS Doc 3134, Jun 2007.

[118] G. D. Barr, T. K. Gaisser, S. Robbins, and T. Stanev, "Uncertainties in Atmospheric Neutrino Fluxes," Phys. Rev., vol. D74, p. 094009, 2006.

[119] G. Battistoni, A. Ferrari, T. Montaruli, and P. R. Sala, "The FLUKA atmospheric neutrino flux calculation," Astropart. Phys., vol. 19, pp. 269290, 2003. [Erratum: Astropart. Phys.19,291(2003)]. 
[120] M. Honda, T. Kajita, K. Kasahara, S. Midorikawa, and T. Sanuki, "Calculation of atmospheric neutrino flux using the interaction model calibrated with atmospheric muon data," Phys. Rev., vol. D75, p. 043006, 2007.

[121] A. Blake, "Results of 2011 atmospheric neutrino analysis: (2) systematic uncertainties," MINOS Doc 8477, Oct 2011.

[122] C. Zeitnitz and T. A. Gabriel, "The GEANT - CALOR interface and benchmark calculations of ZEUS test calorimeters," Nucl. Instrum. Meth., vol. A349, pp. 106-111, 1994.

[123] H. Fesefeldt, "The Simulation of Hadronic Showers: Physics and Applications," 1985.

[124] The MINOS Calibration Group, "Calibration position paper for preshutdown data," MINOS Doc 3941, Mar 2008.

[125] A. Blake, T. Carroll, and W. Flanagan, "Standard oscillations workshop summary (after day 2 of 3)," MINOS Doc 11725, Jan 2017.

[126] A. Blake, S. Cao, L. Corwin, M. M. de Medeiros, and R. Mehdiyev, "Position paper on combining the measurements of the anti-neutrino oscillation parameters from the beam and atmospheric neutrino data sets," MINOS Doc 9019, May 2012.

[127] M. Sanchez, "Nova results and prospects," June 2018. 
[128] D. P. MENDEZ, "First $\nu_{\mu}+\bar{\nu}_{\mu}$ Disappearance Results from the NOvA experiment," June 2018.

[129] P. Adamson et al., "Search for sterile neutrinos in MINOS and MINOS+ using a two-detector fit," Phys. Rev. Lett., vol. 122, no. 9, p. 091803, 2019.

[130] S. De Rijck, A Search for Large Extra Dimensions with MINOS and MINOS+. PhD thesis, Texas U., 2018.

[131] M. A. Acero et al., "First measurement of neutrino oscillation parameters using neutrinos and antineutrinos by NOvA," 2019.

[132] M. Wascko, "T2k status, results, and plans," June 2018.

[133] M. G. Aartsen et al., "Measurement of Atmospheric Neutrino Oscillations at 6-56 GeV with IceCube DeepCore," Phys. Rev. Lett., vol. 120, no. 7 , p. $071801,2018$.

[134] K. Abe et al., "Atmospheric neutrino oscillation analysis with external constraints in Super-Kamiokande I-IV," Phys. Rev., vol. D97, no. 7, p. $072001,2018$.

[135] R. Davis, "Nobel lecture: A half-century with solar neutrinos," Rev. Mod. Phys., vol. 75, pp. 985-994, Aug 2003.

[136] V. D. Barger, K. Whisnant, S. Pakvasa, and R. J. N. Phillips, "Matter Effects on Three-Neutrino Oscillations," Phys. Rev., vol. D22, p. 2718, 1980. 
[137] M. Mesquita de Medeiros, Estudo da Oscilao de Neutrinos Municos Usando Dados Atmosfricos e de Acelerador nos Experimentos MINOS e MINOS+. PhD thesis, Goias U., 2015.

[138] P. Adamson et al., "Primary proton beamline for the fermilab main injector neutrino program," MINOS Doc 1669-v13, Nov 2007.

[139] P. Adamson et al., "Measurement of the Neutrino Mass Splitting and Flavor Mixing by MINOS," Phys. Rev. Lett., vol. 106, p. 181801, May 2011. 


\section{Vita}

Thomas Carroll received his Bachelor of Science from the University of

Pittsburgh in 2014. He started the Ph.D. program in the Department of

Physics at the University of Texas at Austin in August 2014.

Email address: tjcarroll@utexas.edu

This dissertation was typeset with $\mathrm{AT}_{\mathrm{E}} \mathrm{X}^{\dagger}$ by the author.

\footnotetext{
${ }^{\dagger} \mathrm{LT}_{\mathrm{E}} \mathrm{X}$ is a document preparation system developed by Leslie Lamport as a special version of Donald Knuth's $\mathrm{T}_{\mathrm{E}} \mathrm{X}$ Program.
} 